POSITION ESTIMATION OF MOBILE MAPPING IMAGING SENSORS USING AERIAL IMAGERY

Phillipp Leopold Heinz Fanta-Jende 



\section{POSITION ESTIMATION OF MOBILE MAPPING IMAGING SENSORS USING AERIAL IMAGERY}

DISSERTATION

to obtain

the degree of doctor at the University of Twente, on the authority of the rector magnificus, prof.dr. T.T.M. Palstra,

on account of the decision of the Doctorate Board, to be publicly defended on Wednesday, 20 November 2019 at 14:45 hrs

by

Phillipp Leopold Heinz Fanta-Jende

born on April 16, 1988.

in Gräfelfing, Munich, Germany 
This thesis has been approved by

Prof. dr. M.G. Vosselman supervisor

Prof. Dr-Ing. M. Gerke co-supervisor

Dr. F.C. Nex co-supervisor

ITC dissertation number 370

ITC, P.O. Box 217, 7500 AE Enschede, The Netherlands

ISBN 978-90-365-4884-7

DOI $10.3990 / 1.9789036548847$

Cover designed by Phillipp and Johanna Fanta-Jende

Printed by ITC Printing Department

Copyright (c) 2019 Phillipp Fanta-Jende, The Netherlands. All rights reserved. No parts of this thesis may be reproduced, stored in a retrieval system or transmitted in any form or by any means without permission of the author. Alle rechten voorbehouden. Niets uit deze uitgave mag worden vermenigvuldigd, in enige vorm of op enige wijze, zonder voorafgaande schriftelijke toestemming van de auteur.

\section{UNIVERSITY OF TWENTE.}

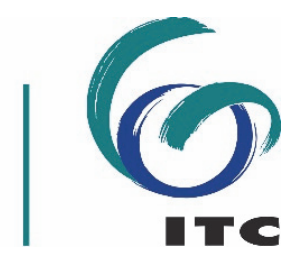


Graduation committee:

Chairman/Secretary

Prof.dr.ir. A. Veldkamp University of Twente

\section{Supervisor}

Prof.dr.ir. M.G. Vosselman

University of Twente

\section{Co-supervisor(s)}

Prof. dr.-ing. M. Gerke

Dr. F.C. Nex

Universität Braunschweig

University of Twente

\section{Members}

Prof.dr.ir. A. Stein

Prof.dr. F.D. van der Meer

Prof.dr.-ing. C.H.R. Heipke

Prof.dr.-ing. H. Mayer

University of Twente

University of Twente

Leibniz Universität Hannover

Universität der Bundeswehr München 



\section{Acknowledgements}

During my time at ITC, I have received great support and assistance. Foremost, I would like to thank my promoter and supervisor Prof. George Vosselman, whose competent guidance and strong expertise made this research work possible. I always was and still am fascinated by Prof. Vosselman's keen perception and attention to detail while never losing the overall perspective.

I would also like to express my deep gratitude to Prof. Markus Gerke who not only imagined the original research idea but was upmost supportive throughout the entire time. His creativity and ability to think laterally were invaluable factors for the successful completion of this research work.

I wish to present my special thanks to my co-supervisor Dr. Francesco Nex whose dedication and diligence were greatly encouraging and of particular importance for me. I enjoyed the countless fruitful discussions with Dr. Nex which helped me to solve seemingly unsolvable problems.

I would also like to show my gratitude to my colleague and dear friend Zille Hussnain. Together, we were able to accomplish this journey. The mutual support and great assistance were driving forces throughout the years and I am thankful that I had such great company.

My sincere thanks also goes to the user committee and partners in the research project, in particular Bart Beers, Peter Joosten and Bashar Alsadik, whose assistance was pivotal for the accomplishment of the project.

In addition, I would like to thank all my fellow colleagues wholeheartedly for the great time and support. It was a pleasure to be working with you!

And finally, last but by no means least, I am deeply grateful to my family. My wonderful wife Johanna who always provided me through invaluable moral and emotional support. My mother Sylvia, my grandmother Walli Wanda and my sister Elena whose unconditional support is one the most precious things in my life. My father Hans-Joachim who introduced me to technical thinking at an early age and my deceased grandfather Udo who encouraged me to pursue a scientific career in the first place. My parents-in-law Hilde and Walter who are fantastic listeners and fascinating examples always helping in word and deed. Thank you and all the other family members for your encouragement. 


\section{Table of Contents}

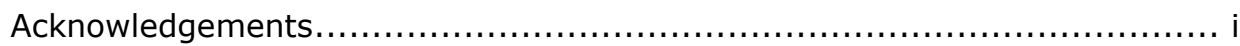

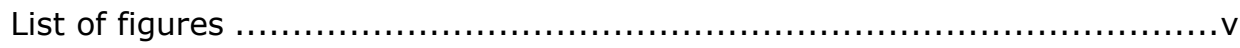

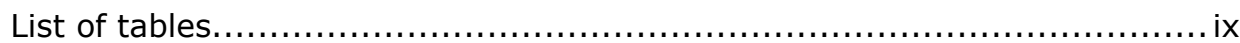

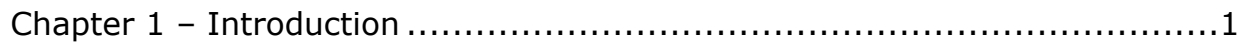

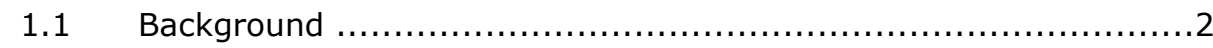

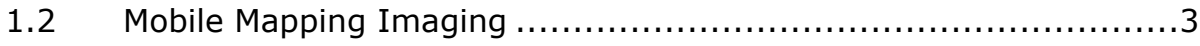

1.3 Limitations of Satellite-based Positioning Solutions ...................

1.4 Current mitigation approaches for GNSS-denied environments ......6

1.5 Research Framework and Objectives...................................

$1.6 \quad$ Structure of the thesis ...................................................

Chapter 2 - Investigating different feature extraction methods ................13

2.1 Part 1 - Low-level tie feature extraction of mobile mapping data (MLS/images) and aerial imagery ............................... 14

$2.1 .1 \quad$ Abstract........................................................... 14

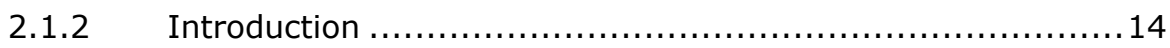

2.1.3 Project Overview................................................. 15

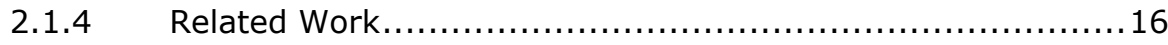

2.1.5 Low-Level Tie Feature Extraction ............................. 17

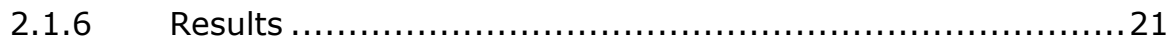

2.1.7 Discussion..................................................... 33

2.2 Part 2 - Advanced tie feature matching for the registration of mobile mapping imaging data and aerial imagery .....................34

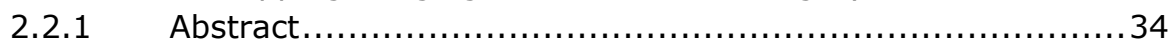

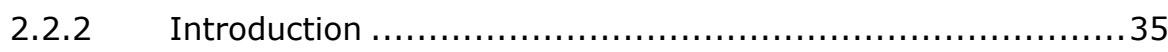

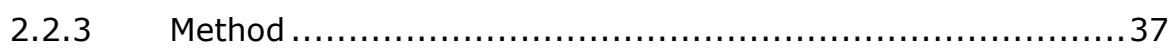

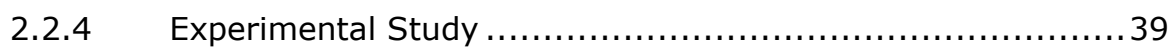

$2.2 .5 \quad$ Conclusion ..................................................... 49

Chapter 3 - A fully automatic approach to register mobile mapping and airborne imagery to support the correction of platform trajectories in GNSS-

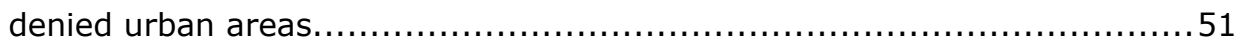

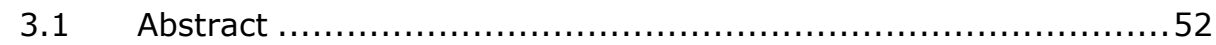

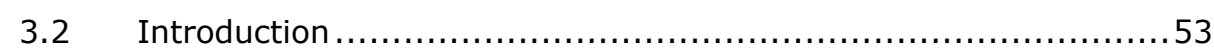

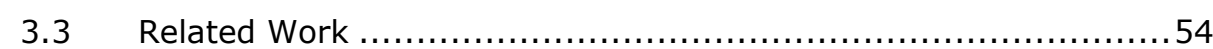

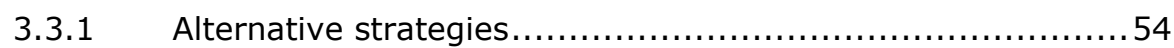

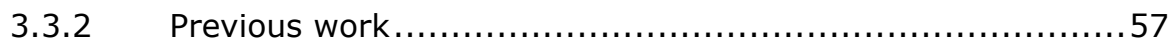

3.4 Methodology of the registration..................................... 58

3.4.1 Overview ......................................................... 58

3.4.2 Processing along the trajectory ...............................60

3.4.3 Ortho-projection / Inverse perspective mapping ...............60

3.4.4 Matching panoramic images to obtain 3D points ..............6 62

3.4.5 Matching panoramic images with aerial reference data ........65

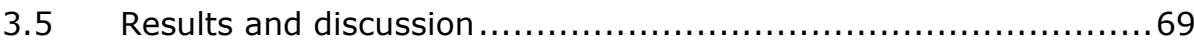




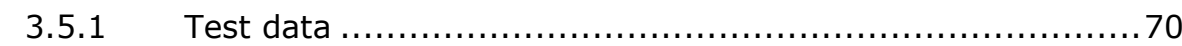

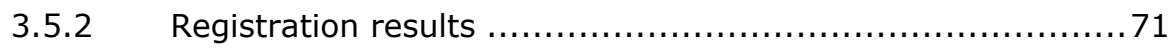

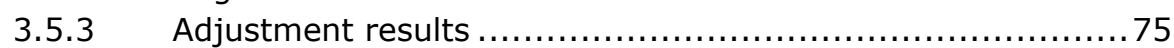

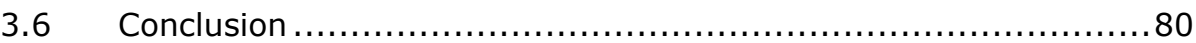

Chapter 4 - Co-registration of panoramic mobile mapping images and oblique

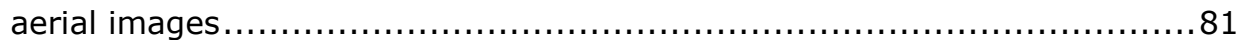

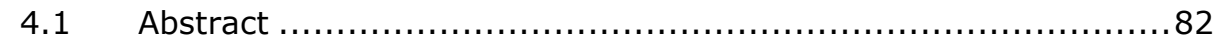

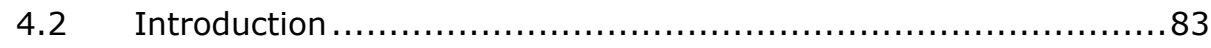

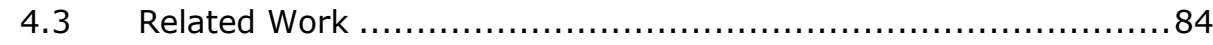

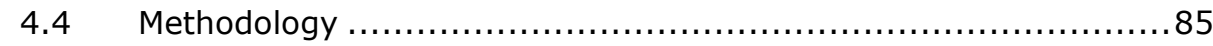

4.4.1 Sparse Point Cloud from Mobile Mapping Images ............... 86

4.4.2 Plane Fitting to Identify Façades ............................... 89

4.4.3 Visibility Hypothesis....................................... 90

4.4.4 Point Cloud Thinning ........................................... 92

4.4.5 Image to Plane Projection ......................................... 93

4.4.6 Registration................................................ 95

4.4.7 Outlier Removal ........................................... 96

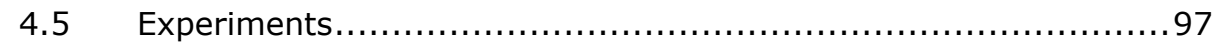

4.5.1 Maximum Angular Distance to Reference Vector for Plane

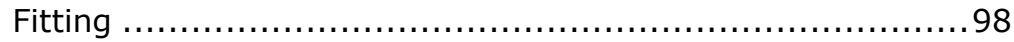

4.5.2 Minimum Number of Points for Plane Fitting .................. 100

4.5.3 Mutual Information to Compute Initial Transformation ....... 103

4.5.4 Hierarchical Matching and Patch Size........................ 105

4.5.5 Outlier Removal ........................................... 107

4.6 Discussion and Conclusion......................................... 109

Chapter 5 - Correction of mobile mapping trajectories in GNSS-denied environments using aerial nadir and aerial oblique images ................. 111

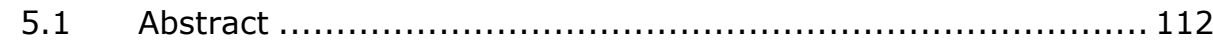

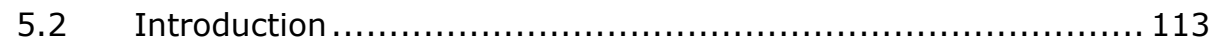

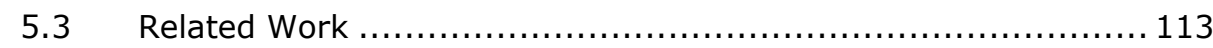

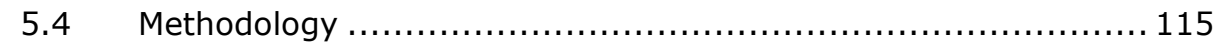

5.4.1 Co-registration of aerial nadir and mobile mapping images. 115

5.4.2 Co-registration of aerial oblique and mobile mapping

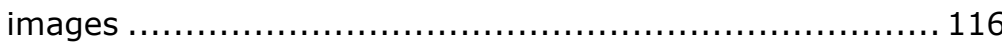

5.4.3 Mobile mapping data adjustment ............................ 117

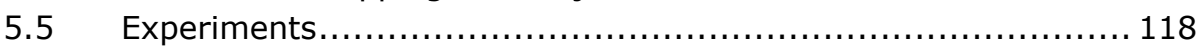

5.5.1 Adjustment results using correspondences to the aerial nadir images ............................................... 120

5.5.2 Adjustment results using correspondences to the aerial

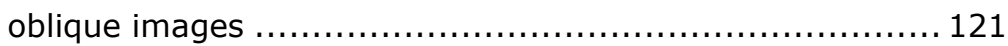

5.5.3 Adjustment results using correspondences to the aerial

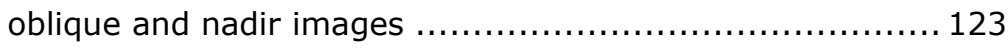

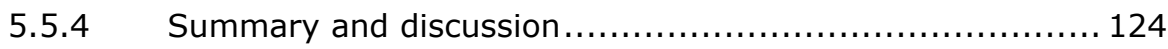

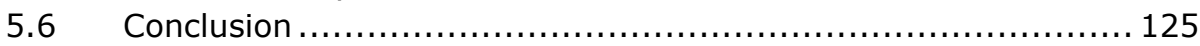


5.7 Annex - Adjustment with deteriorated data .................... 126

Chapter 6 - Synthesis.................................................. 131

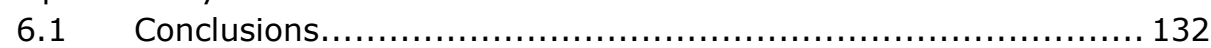

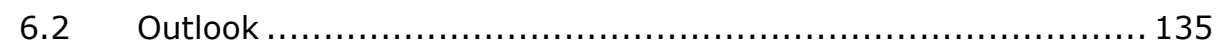

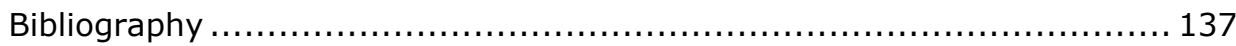

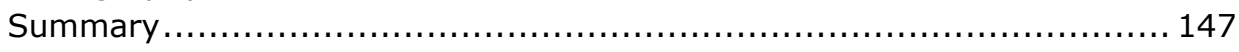

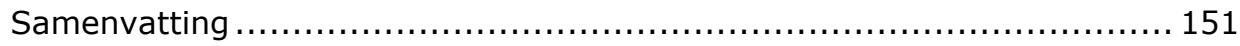




\section{List of figures}

Figure 1.1 Schematics of an early mobile mapping system (VISAT) depicting the camera and IMU coordinate system, El-Sheimy and Schwarz (1993). ...3

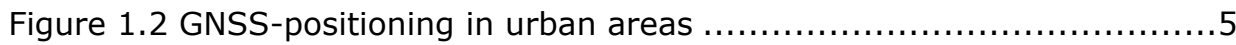

Figure 1.3 Schematic overview of the thesis' chapters. ..................... 10

Figure 2.1 Mobile mapping panoramic image in equirectangular projection. 18

Figure 2.2 Panoramic image projected onto an artificial ground plane. ...... 19

Figure 2.3 Point cloud patch (left) to ortho-image conversion (right)......... 21

Figure 2.4 Four subsets of a typical urban scene (coloured tiles from scene 1

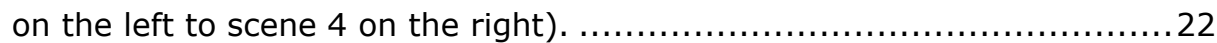

Figure 2.5 SIFT keypoints detected in aerial image (left), panoramic image (centre) and MLS intensity image (right). ............................... 23

Figure 2.6 KAZE keypoints detected in aerial image (left), panoramic image (centre) and MLS intensity image (right) . ............................ 23

Figure 2.7 AKAZE keypoints detected in aerial image (left), panoramic image (centre) and MLS intensity image (right). .............................. 24

Figure 2.8 Förstner keypoints detected in aerial image (left), panoramic image (centre) and MLS intensity image (right). ............................... 24

Figure 2.9 Matched LATCH keypoints in the first scene and first iteration....26 Figure 2.10 Comparison of matching results of AKAZE (top), KAZE (centre) and SIFT (bottom) in 3rd run of the 1 st scene. ..............................27

Figure 2.11 Matched SIFT keypoints in the second scene and first iteration

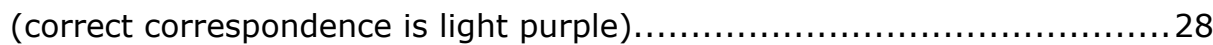

Figure 2.12 Matched SIFT keypoints in the second scene and second iteration. .28

Figure 2.13 Matched KAZE keypoints in the second scene and third iteration. 29

Figure 2.14 Comparison of matching results of AKAZE (top), KAZE (centre) and SIFT (bottom) in 4 th run of the 2 nd scene. ...............................29

Figure 2.15 Comparison of SIFT (top) and KAZE (bottom) in 4th run on 1st

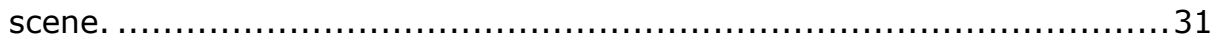

Figure 2.16 Matching results of AKAZE (top) and KAZE (bottom) in 4th run on scene 2.

Figure 2.17 Left: aerial image with extracted Förstner keypoints; Right: aerial image's keypoints back-projected into MM image. Coloured circles illustrate the horizontal error. Moreover, the ambiguity problem with repetitive road markings becomes apparent using the example of the green circle; three corners have been identified in the aerial image. The back-projected keypoints, however, are now closer to the adjacent square-shaped road marking which may lead to a wrong correspondence.

Figure 2.18 Test site in Rotterdam (the gap is caused by a building spanning the road). .40

Figure 2.19 Matching results of proposed method across all 14 tiles..........42 
Figure 2.20 Matching results of AGAST detection and SURF description across

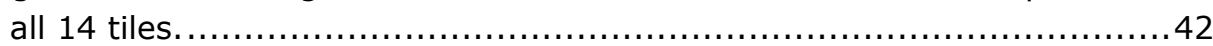

Figure 2.21 Matching results of Förstner detection and SURF description across

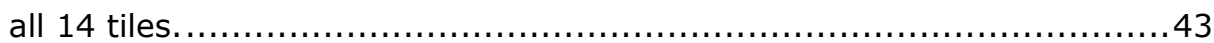

Figure 2.22 AGAST/SURF matching result of 2 nd tile. ...................... 44

Figure 2.23 Förstner Cross-Correlation matching result of 2 nd tile. ...........44

Figure 2.24 Förstner/SURF matching result of 2 nd tile. ....................... 45

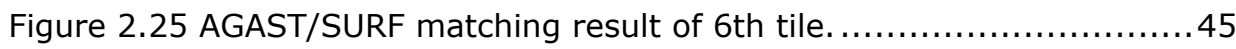

Figure 2.26 AGAST keypoints in aerial nadir and MM ortho-image............ 46

Figure 2.27 Förstner Cross-Correlation matching result of 6th tile.............46

Figure 2.28 Förstner Cross-Correlation matching result of 8 th tile............47

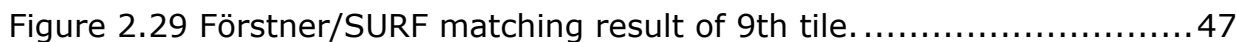

Figure 2.30 Förstner Cross-Correlation matching result of 9th tile............48

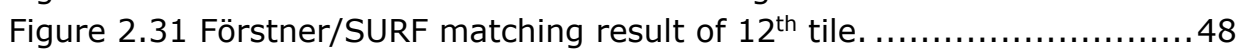

Figure 2.32 Förstner Cross-Correlation matching result of $12^{\text {th }}$ tile............49

Figure 2.33 AGAST/SURF matching result of 13 th tile with not a single correct correspondence. ............................................................ 49

Figure 3.1 Left: Non-line-of-sight problem; Right: Multipath interference

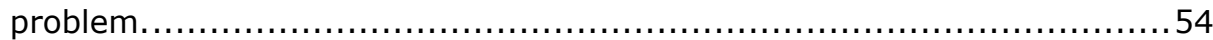

Figure 3.2 Design of the registration procedure [reference to respective section

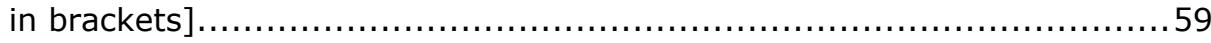

Figure 3.3 Principle of ortho-projection. ..................................61 Figure 3.4 Ortho-projected MM image with masked bonnet. Please note the highly distorted silver car in the bottom half of the image or the traffic light's post. These distortions are unfavourable side effects of the ortho-projection.

Figure 3.5 Aerial and MM image patches; bottom: Aerial and MM image patch after Wallis filtering - the output is always a greyscale image.

Figure 3.6 Top: Exemplary registration results: Normalised cross-correlation template registration of a MM ortho-image (left) and an aerial image (right). Bottom: Phase-correlation registration. Please note: this is a result without

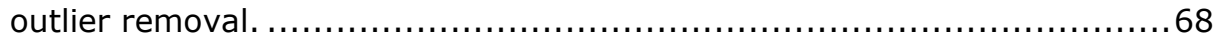

Figure 3.7 Mobile Mapping test areas in Rotterdam with coordinate axes in the RD New system. $[$ Red $=$ Area $1 ;$ Green $=$ Area $2 ;$ Blue $=$ Area 3$] \ldots \ldots . . . .71$

Figure 3.8 Localisation limitations due to differing image properties; Left: MM image, right: Aerial image; Overlay: the red mark indicates the identified correspondence, the blue mark the presumed correct position. Thus, the correspondence has an offset of more than a pixel........................ 73

Figure 3.9 Registration result of two different aerial images. Red circle depicts correspondence which is not at the same location with an offset of about a pixel. Remaining correspondences are accurate. 74

Figure 3.10 Cropped registration example: Imprecise feature locations in aerial images; from left to right: $M M$ image, $1^{\text {st }}$ aerial image, same $M M$ image, $2^{\text {nd }}$ 
aerial image; Please note: the horizontal red lines in the figure have been manually added to make a comparison easier. .......................... 74

Figure 3.11 Successful registration result between $\mathrm{MM}$ and aerial image, although strong illumination differences are present. Please note: Correspondences are initially identified between two MM images, thus other road markings other than the triangular ones in this example were not in the overlapping area of the source MM images.............................. 75

Figure 3.12 Left: Wrong matches due to repeated patterns; Right: Correct correspondences with a different aerial image $\ldots \ldots \ldots \ldots \ldots \ldots \ldots \ldots \ldots \ldots \ldots . \ldots 75$

Figure 3.13 GCP measurement example. Red: GCP projected into MM image using original orientation; blue: GCP projected into MM image using updated orientation; Yellow: Actual position of GCP measured manually.... .77

Figure 3.14 Error comparison of original and adjusted Area 1 compared to one GCP; left part: multi-view triangulation; right part: two-view triangulation.

78

Figure 3.15 RMSE comparison of original and adjusted Area 2 compared to three GCPs; left part: multi-view triangulation; right part: two-view triangulation. .......................................................... 78

Figure 3.16 RMSE comparison of original and adjusted Area 3 compared to three GCPs; left part: multi-view triangulation; right part: two-view

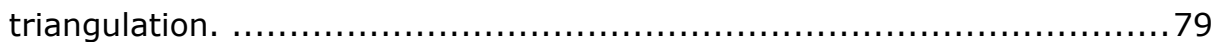

Figure 4.1 Mobile mapping (MM) to aerial oblique image registration pipeline.

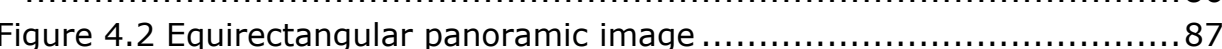

Figure 4.3 (a) Yaw deviations for perspective image creation. (b) Principle of sparse point cloud generation based on image triplets. ...................... 88

Figure 4.4 Example of a perspective image triplet for sparse point cloud generation with respective correspondences. From left to right, images 1, 2 and 3 . .88

Figure 4.5 A façade occluded by vegetation. (a) Point cloud (purple points), recording locations (red circles edged in black), fitted plane (red). (b) Oblique aerial image of the scene, where the red circle indicates the plane centre in the scene.

Figure 4.6 Principle of façade visibility for oblique aerial images. .............91

Figure 4.7 An example of a façade which is not matchable, since the awning above the windows in the left image is occluding most of the patch........92

Figure 4.8 (a) Schematics of patch creation. The eigenvectors (blue and green) and the plane's normal vector (red) constitute the local coordinate system, where the object point (black) on the plane (cyan) is defined as the centre of the discretised grid (grey). (b) Example of a grid (central area in yellow) projected into a panoramic image. 94

Figure 4.9 Six pairs of oblique aerial (left side of every pair) and panoramic (right side) image patches of the same grid. The oblique aerial image patches are retrieved from different oblique aerial images, hence the partially 
occluded part. Note the different resolutions of the oblique aerial image; however, the panoramic image in a pair shares the same sampling. .......95

Figure 4.10 MM image patch (a) with corresponding oblique aerial patch (b). Due to the low resolution of the oblique aerial patch, this pair cannot be used for registration. .95

Figure 4.11 Trajectory of MM recording locations in Rotterdam. .............. 97

Figure 4.12 Number of correspondences (ordinate) with different maximum angular distance (abscissa) for plane fitting. ............................ 99

Figure 4.13 Wrong plane estimation leads to skewed image patches........ 100

Figure 4.14 Number of correspondences with different number of points per plane. 100

Figure 4.15 Example of correspondences between two triplets of the same location. (Top: right-hand side of trajectory; bottom: left-hand side.) .... 102

Figure 4.16 Distribution of triplet correspondences across the entire trajectory for both sides. Top row: correspondences on the right-hand side of the trajectory. Bottom row: correspondences on the left-hand side. Note the equal distribution as well as the slanted pattern on the side views representing façades. 102

Figure 4.17 Influence on minimum number of points per plane; a comparison of recording locations with (green) and without (red) correspondences. From left to right: 10 points (default), 5 points, 20 points and 30 points. See also Figure 4.11 . 103

Figure 4.18 Comparison between fine registration results of mutual information and normalised cross correlation for three examples (top, middle, bottom). In each example: left image is the MM patch; middle image is the MI registration result in the oblique patch; right image is the NCC registration result in the oblique patch. 105

Figure 4.19 Number of correspondences with different patch sizes and active/inactive hierarchical matching..................................... 106

Figure 4.20 Large image patch size of $12 \mathrm{~m}$. ............................... 107

Figure 5.1 Left: Aerial nadir image, right: re-projected MM image. .......... 116

Figure 5.2 Left: Mobile mapping image patch, right: aerial oblique image patch.

Figure 5.3 Schematics of the selected adjustment method.

Figure 5.4 Characteristics of the four test areas (only subsets). Area 1 (green trajectory), area 2 (red traj.), area 3 (blue traj.), area 4 (yellow traj.). The recording locations and surveyed GCPs in the selected subset have been projected into an overlapping aerial oblique image. 119

Figure 5.5 Distribution of correspondences (green) of area 2 along the trajectory (white); [rotated by 90 degrees]........................... 120

Figure 5.6 Distribution of correspondences (green) of area 3 along the trajectory (white). All the correspondences were identified on one side of the road. 
Figure 5.7 Distribution of correspondences in area 1. From top to bottom: aerial oblique correspondences, aerial nadir correspondences, and both combined.

\section{List of tables}

Table 2-1 Number of combined keypoints over all subsets per detection

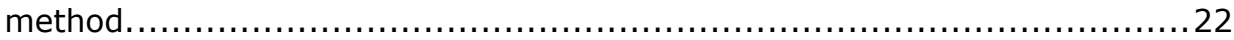

Table 2-2 Matching results of scene 1 between aerial and panoramic image of the 1 st and 2 nd iteration. .................................................... 26 Table 2-3 Matching results of scene 1 between aerial and panoramic image of

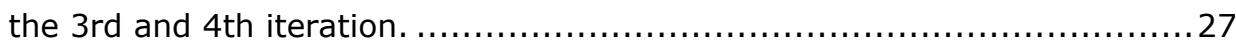
Table 2-4 Matching results of scene 2 between aerial and panoramic image of

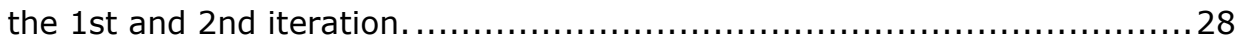
Table 2-5 Matching results of scene 2 between aerial and panoramic image of

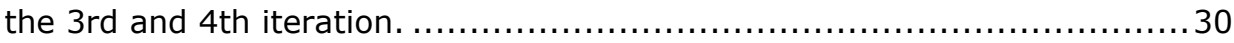
Table 2-6 Matching results of scene 1 between aerial and MLS ortho-image of

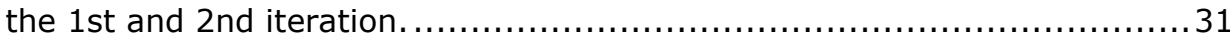
Table 2-7 Matching results of scene 1 between aerial and MLS ortho-image of

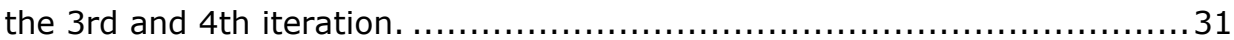
Table 2-8 Matching results of scene 2 between aerial and MLS ortho-image of

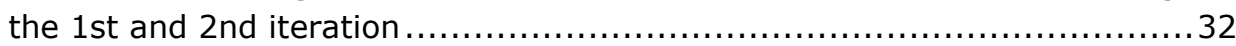
Table 2-9 Matching results of scene 2 between aerial and MLS ortho-image of

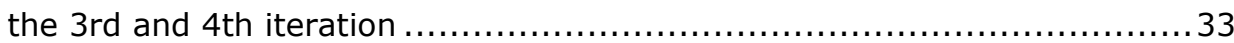
Table 2-10 Summary of matches, inliers and averages of all test tiles.......41 Table 3-1 Overview of registration results of test areas in Rotterdam........72 Table 3-2 Position updates after adjustment of all three MM test trajectories. .76

Table 3-3 Error comparison of original and adjusted Area 1 compared to one GCP (please note: no RMSE, as only one GCP available). ..................77 Table 3-4 RMSE comparison of original and adjusted Area 2 compared to three

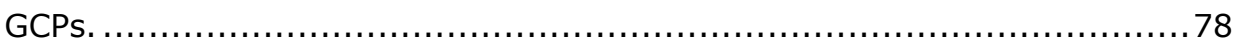
Table 3-5 RMSE comparison of original and adjusted Area 3 compared to three

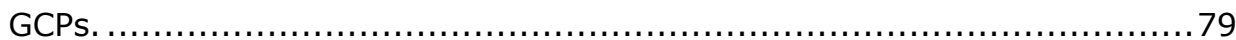
Table 4-1 Parameters for different experiments. NCC is normalised cross correlation; MI is mutual information. Entries in bold represent differences from the default setting. .98

Table 4-2 Results for the default and experiments 1 to 4 [different threshold values for maximum angular distance for plane fitting]. Bold entries represent the best result. 99 Table 4-3 Parameters for the default and results of experiments 5 to 7 [different minimum number of points for plane fitting]. Bold entries represent the best result. 101 
Table 4-4 Statistics of correspondences between perspective views of the entire trajectory....................................................... 101

Table 4-5 Results for the default and experiments 8 and 9 [initial transformation/fine registration with MI or NCC only]. Bold entries represent the best result. 104

Table 4-6 Results for the default and experiments 10 to 14 [different image patch sizes and hierarchical matching]. Bold entries represent the best result.

Table 4-7 Comparison of inlier rate before and after outlier removal. Bold entries represent the best result. 108 Table 4-8 Rejection threshold for default parameter set. The last column shows results with consensus-tracking activated. Bold entries represent the best result. 108

Table 5-1 Overview of the four test areas. .................................. 120

Table 5-2 Adjustment result using only correspondences to the aerial nadir images [in metres]. Best result in bold. .................................. 120 Table 5-3 Exemplary trajectory updates at two check points in area 4 . Best result in bold [in metres].

Table 5-4 Adjustment result using only correspondences to the aerial oblique images [in metres]. Best result in bold.

Table 5-5 Statistics of the differences before and after the adjustment of area 1 with correspondences to the aerial oblique images [in metres]. 121 Table 5-6 Statistics of the differences before and after the adjustment of area 3 with correspondences to the aerial oblique images [in metres]. 122 Table 5-7 Adjustment result using correspondences to the aerial oblique and nadir images [in metres]. Best result in bold.

Table 5-8 RMSE combined in $\mathrm{X}, \mathrm{Y}$ before and after respective adjustments. Best result in bold [in metres]. Borderline cases not rounded. 125 Table 5-9 RMSE combined in $X, Y, Z$ before and after respective adjustments. Best result in bold [in metres]. Borderline cases not rounded....

Table 5-10 Example results for proposed residual calculation methods [in

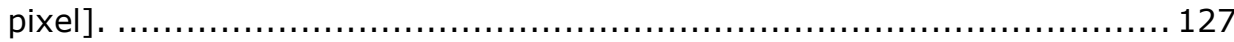
Table 5-11 Results of a horizontal adjustment using different residual computation methods [in metres].... 128 Table 5-12 Results of a full adjustment using different residual computation methods [in metres]. 128

Table 5-13 Mean and standard deviation of differences between triangulated aerial oblique correspondences and mobile mapping correspondence in object space [in metres]. 26 tie points in total. 129 
Chapter 1-Introduction 


\subsection{Background}

Data is the very core of every geospatial application. Rapid developments in technology and the increasing adoption of geospatial solutions let new businesses emerge which pushed the diversification of both, acquisition and application, to new limits.

Traditional data capture methods, such as aerial photogrammetry, surveying, or satellite-based approaches could be augmented and complemented by new sensor systems, instruments, and platforms. Particularly, the combination of developments in multi-sensor and multi-platform solutions paved the way for an array of intriguing acquisition technologies. A multitude of sensor systems can now be installed on aerial, terrestrial, and water-based vehicles alike while the platform's location can be accurately determined.

A prominent example where these developments all come into play is mobile mapping. By carrying sensors on a moving platform to collect data for all sorts of geospatial data products, such as maps, images, videos, or GIS ${ }^{1}$, mobile mapping can be described as an inventory system (Hofmann-Wellenhof et al., 2003). In this case, positioning may not be the primary task but it is an indispensable necessity to enable mobile mapping's actual functionality - the georeferencing of its surrounding.

Hence, the inception of mobile mapping is closely related to the developments in the field of direct georeferencing technologies. 1993, when GPS achieved its initial operational capability, first experimental mobile mapping systems emerged (El-Sheimy et al., 1993, Cosandier et al., 1993). Certain criteria had to be fulfilled in order to achieve that goal. Price and size of sensors and instruments were limiting factors in the past, notably reliable georeferencing equipment, i.e. inertial measurement units (IMU) and GNSS²-receivers. Early systems, in particular the component for inertial navigation, were rather expensive and developments in the last 15 years in the area of MEMS $^{3}$ could enable reliable and accurate direct georeferencing with a lower price tag (Schwarz et al., 2004).

The absolute position is determined by GNSS while the attitude and relative movement from a position fix is derived from IMU readings and in some architectures by DMIs ${ }^{4}$ as well. This integrated system for direct georeferencing

\footnotetext{
${ }^{1}$ Geographic Information System

${ }^{2}$ Global Navigation Satellite System

3 Micro Electronic Mechanical Systems

${ }^{4}$ Distance Measuring Instrument
} 
allows the platform and thus mounted imaging or lidar ${ }^{5}$ sensors to compute its absolute position and orientation at all times.

\subsection{Mobile Mapping Imaging}

In theory, the term mobile mapping encompasses all forms of geospatial data acquisition using a mobile platform carrying one or more sensor systems (Tao and $\mathrm{Li}, 2007$ ). Although this involves for instance aerial laser scanning and aerial photogrammetry as well as the georeferencing of images taken by a mobile phone camera, a typical domain for mobile mapping is terrestrial data acquisition using cameras or laser scanners mounted on a car. The aforementioned first mobile mapping systems followed a similar design (see Figure 1.1).
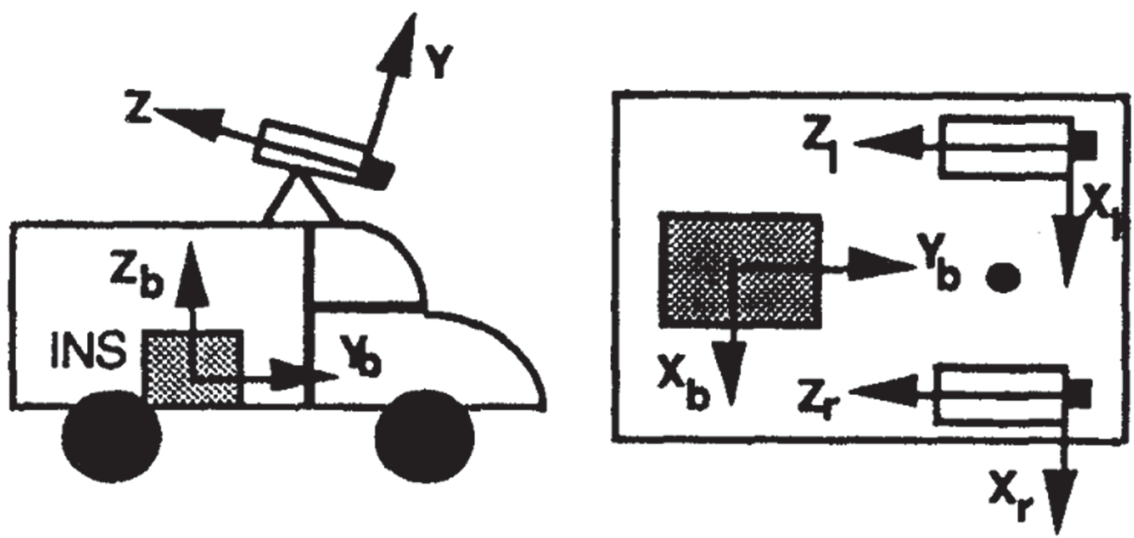

Figure 1.1 Schematics of an early mobile mapping system (VISAT) depicting the camera and IMU coordinate system, El-Sheimy and Schwarz (1993).

Besides the georeferencing component, a mobile mapping system employs cameras or lidar sensors whose position is accurately calibrated with respect to the positioning instruments, referred to as the boresight alignment (cf. Angelats and Colomina (2014); Kersting et al. (2012)). In case of a mobile mapping camera system, this step relates the mapping reference frame to the camera coordinate system. Mobile laser scanning or hybrid systems are not part of this work, but a comprehensive review of this technology can be found in Puente et al. (2013).

The camera itself is also required to be calibrated in order to determine its internal geometry. Independently from the camera system, the principal point, the focal length, and the lens distortion parameters are identified.

\footnotetext{
${ }^{5}$ Light detection and ranging
} 
Consequently, acquired image data is oriented, i.e. the extrinsic and intrinsic orientation parameters of the camera are known at the time of the acquisition.

In practice, there are multiple camera systems for mobile mapping in use. Single (perspective) camera systems are mostly employed for specific tasks, e.g. MonoSLAM (Migliore et al., 2009) or affordable road inventory systems (Gontran et al., 2007). Other designs operate with two or more cameras, which enables stereo vision (Burkhard et al., 2012; Jia et al., 2003; Tournaire et al., 2006). The most frequently used setup in mobile mapping imaging is employing panoramic cameras. Notable examples are summarised in the review of Payá et al. (2017). Panoramic cameras, also denoted as omnidirectional cameras, have the advantage to cover the entire surrounding (360 degrees) of the moving platform unlike other setups which capture a narrower and directed field of view. This enables a greater overlap between adjacent recording locations and thus a more stable mutual feature space for methods such as visual odometry. For instance, panoramic images can be produced by the combination of a regular perspective camera with a convex mirror which maps the surrounding onto the image plane or multiple cameras with a certain overlap (Scaramuzza, 2014). The latter method is used for creating the panoramic images used in this work (van den Heuvel et al., 2006).

In any case, a 360 degrees view leads to distortions if mapped onto a twodimensional image plane. This relationship is most commonly embedded by using a cylindrical, spherical or rectilinear projection. Cylindrical equidistant projections, in particular equirectangular projections, are used in this work. This image geometry is based on an aspect ratio of $2: 1$, featuring 360 degrees on the horizontal (longitude) and of 180 degrees on the vertical (latitude) axis. The meridians are projected equally spaced and parallel which results in a nonconformal and non-equal-area map projection. Although this projection is not very useful for navigation or map making, it allows for an easy conversion between image and spherical coordinates since the angular resolution is directly linked to the pixel spacing (Snyder, 1993).

Mobile mapping systems carrying imaging equipment are nowadays widely used. The mobile mapping imaging data in this work has been provided by CycloMedia6, a company that developed its own mobile mapping system employing five cameras set up in an array. These cameras take a front, left, right, back, and top image exactly at the same position which enables a parallax-free and high-resolution panoramic image.

${ }^{6}$ CycloMedia BV; https://www.cyclomedia.com 


\subsection{Limitations of satellite-based positioning solutions}

Although the design of such a system proved to be reliable, direct georeferencing has its caveats. GNSS relies on pseudorange measurements between the receiver and navigation satellites. The position of the receiver is computed by the difference between the time of arrival and the time of transmission, denoted as the time of flight. Satellite-based positioning is prone to signal outages, which particularly occur in urban areas where tall buildings and other structures obstruct the direct line-of-sight between the GNSSreceiver and the navigation satellites. To this end, mobile mapping platforms are mostly operated in an integrated mode where GNSS and inertial sensor are used in conjunction to bridge these signal outages (Tao, 2000). In such a scenario, the inertial navigation sensors propagate the last GNSS position fix until the platform's position can be determined again once the direct line-ofsight is restored. If a signal outage persists over a longer period, the position accuracy may deteriorate due to drift effects of the inertial sensors. A related issue with respect to satellite-based positioning is called multipath. Since the position of the platform is computed based on the time of flight of the signal, any path that is not the shortest, i.e. direct, between a satellite and the receiver leads to a wrong position estimate. This effect comes into play if the GNSS signal is reflected or diffracted from an object or surface, e.g. a building façade, before it is received by the platform's instruments. This usually occurs in conjunction with other, also direct, signals, hence the name multipath (see Figure 1.2).

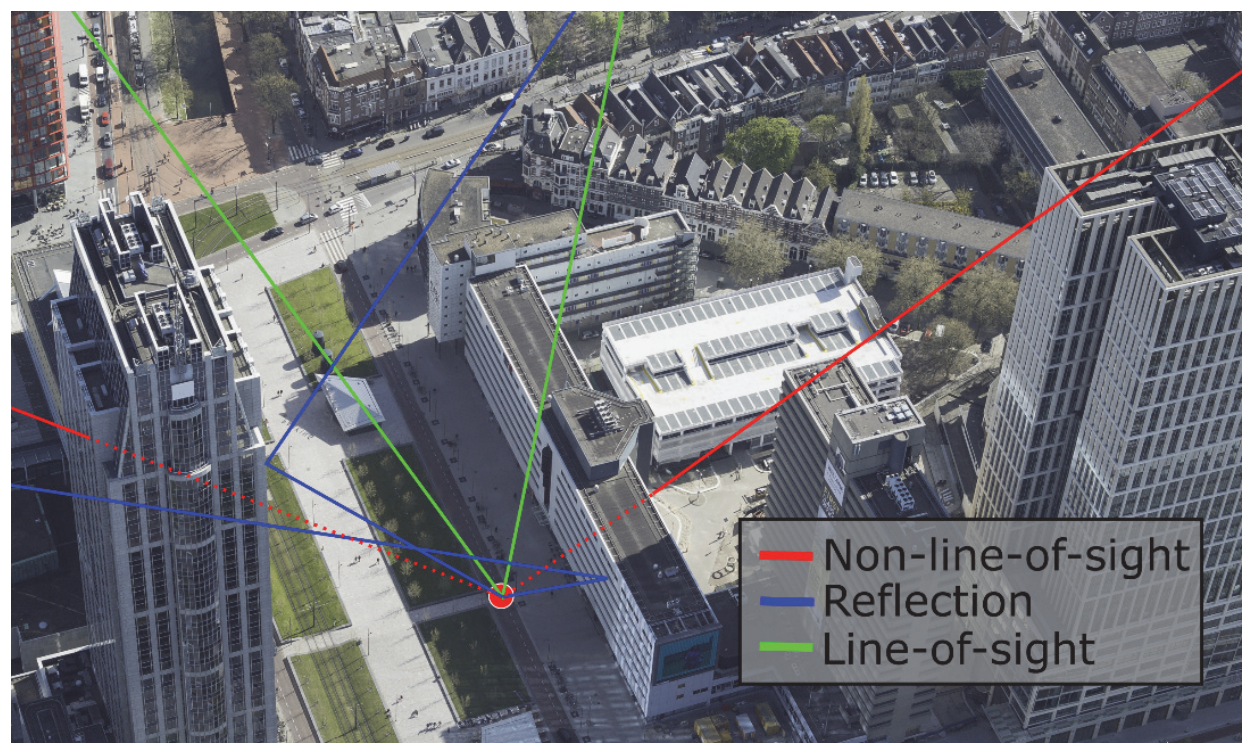

Figure 1.2 GNSS-positioning in urban areas. 
Consequently, the mobile mapping platform's position accuracy can be deteriorated by a couple of metres. Although there are certain strategies to mitigate multipath effects, a reliable prevention is not possible, as the error source is local and depends on the receiver environment (Kos et al., 2010).

\subsection{Current mitigation approaches for GNSS- denied environments}

GNSS-induced positioning issues do not only affect mobile mapping but also applications in robotics, autonomous driving, navigation, and of course surveying. Whereas autonomous driving and robotics in general require realtime capability in this matter, mobile mapping focuses on data acquisition, which potentially allows for post-processing. Another distinction can be drawn with respect to the integration of external data. Real-time methods for the improvement of trajectories, for instance, have to rely on techniques, such as visual odometry or SLAM, which do not necessarily require data other than onboard sensor readings (see e.g. Balazadegan Sarvrood et al. (2016); Carlone and Karaman (2017); Zhang and Singh (2015)).

These approaches, however, cannot fully compensate for GNSS-induced positioning errors and do not reach consistent sub-metre accuracies in such scenarios. To this end, external data, such as building models, digital maps, ground control points, or aerial images etc. can be used to correct platform trajectories and/or collected data. Depending on the application, different external data is used. For instance, building models can be employed to understand whether the angle of incidence of a GNSS-signal is plausible, i.e. not obstructed or reflected. This technique is usually referred to as 'shadow matching', since multiple candidate positions per epoch are compared to possible satellite visibility predictions (Groves, 2011). Similarly, other methods introduce digital maps into the correction procedure. By registering onboard sensor data with map information, these approaches reach lane-level accuracy in GNSS-denied environments (Gruyer et al., 2014; Roh et al., 2016).

As mentioned earlier, if the primary goal is correcting acquired data, postprocessing techniques can come into play. In photogrammetry, aerial images are usually acquired and oriented with direct sensor orientation. To achieve the highest accuracy possible, ground control points are used. Analogously, this technique can also be employed for mobile mapping data (Cavegn et al., 2016). Depending on the quality, the distribution, and the number of ground control points, sub-decimetre accuracy in GNSS-denied areas can be achieved. However, the acquisition of ground control points and their integration into the correction procedure are costly and labour-intensive. Moreover, a single mobile mapping image covers only a small area compared to an aerial image, hence 
a considerable number of ground control points is needed to improve the mobile mapping data in an area of similar size to an aerial campaign.

A viable, more cost-efficient but similarly accurate technique is the direct utilisation of aerial nadir and oblique images as the source of reference. As aerial surveys employ calibrated cameras, accurate localisation instruments, and do not suffer from GNSS-induced positioning issues, aerial images have highly accurate interior and exterior orientation elements. In conjunction with a few ground control points and depending on the configuration of the aerial campaign, low-centimetre as well as sub-decimetre accuracy of the aerial images' positions is achievable.

In this case, the correction of mobile mapping data requires a registration with aerial images. This relates both image data sets to each other and enables the adjustment of the mobile mapping data. The registration of the data sets is, however, a challenging task due to the overall differences between the image data sets. In current literature, registration approaches rely on aerial nadir images which entail the identification of mostly ground-based features that are salient in both data sets, such as road markings (Azimi et al., 2018; Berveglieri and Tommaselli, 2015; Fischer et al., 2018; Javanmardi et al., 2017). Despite the high accuracy which is potentially achievable with these methods, groundbased features may be occluded in the aerial nadir image or not even present in certain areas.

\subsection{Research Framework and Objectives}

This research is funded by the Dutch Technology Foundation TTW (formerly STW) and is conducted in close cooperation with a user committee consisting of private companies - CycloMedia $^{7}$, Fugro $^{8}$, Slagboom en Peeters ${ }^{9}$, and Topcon $^{10}$ - as well as governmental agencies - the Dutch Kadaster ${ }^{11}$ and Het Waterschapshuis ${ }^{12}$ -

This research work's primary objective is to improve positions of mobile mapping data in GNSS-denied areas by using aerial images as the source of reference. The project is divided into two parts with respect to the mobile mapping data type - mobile mapping imaging and mobile mapping laser scanning. The latter research work is conducted by Zille Hussnain under the supervision of Sander Oude Elberink and George Vosselman in the same department at the Faculty ITC.

7 https://www.cyclomedia.com

8 https://www.fugro.com/

9 https://www.slagboomenpeeters.com

10 https://www.topconpositioning.com/

11 https://www.kadaster.nl/

12 https://www.hetwaterschapshuis.nl/ 
The work dealing with mobile mapping images is separated into the following objectives:

\section{Exploring different possibilities for a reliable feature extraction method}

Mobile mapping and airborne images depict a scene from entirely different perspectives. A registration with off-the-shelf detectors and descriptors is prone to mismatches and leads to inaccurate or wrong registration results. This objective deals with finding a strategy to identify repeatable and re-identifiable features in both, the terrestrial and aerial data set, which are salient enough for a reliable registration. Since aerial nadir and oblique images do not share the same properties with respect to their similarity with mobile mapping images, the criteria for useful features are different. Hence, two individual strategies need to be devised to identify mutual features between (1) mobile mapping and aerial nadir images and (2) mobile mapping and aerial oblique images.

2. Development of an accurate registration method for mobile mapping and aerial nadir imagery

Aerial nadir images are a standard product in the geo-data family. This objective extends the first objective with respect to the specific criteria of this image class. Although aerial nadir and mobile mapping images have different image geometries, certain areas offer an overlap, e.g. the ground. Consequently, strategies need to be found to exploit this relationship for a successful registration. In order to increase the similarity between both data sets, reprojection mechanics are investigated. Moreover, ground-based features, such as road markings may be useful for a registration but can be repeated and hence lead to ambiguities. Additionally, the accuracy of the mobile mapping images may be deteriorated due to GNSS-induced positioning errors but strategies employing search constraints can be useful to decrease the amount of possible matching candidates.

3. Development of an accurate registration method for mobile mapping and aerial oblique imagery

Similar to the aerial nadir case, this objective investigates particularities of the registration between the aerial oblique and mobile mapping images. In contrast to aerial nadir images, aerial oblique images have a slanted view on the scene. Consequently, overlap with aerial oblique images may be present at vertical surfaces, such as façades. Techniques to extract these surfaces as well as strategies to homogenise the image geometries need to be developed in this case. Moreover, aerial oblique images pose a challenge with respect to their visibility. Since a slanted view on a scene may lead to occlusions due to 
vegetation or other buildings, methods need to be found to analyse whether a particular terrestrial and aerial image can be registered.

\section{Design of an adjustment technique for trajectory correction}

There are multiple possibilities to correct mobile mapping data with the registration result obtained from the previous steps. There are two main groups, filtering and bundle adjustment. A filtering approach applies corrections on a trajectory level whereas an adjustment finds a solution for the orientations of the images that are part of the block. Depending on the application and use case, a choice has to be made. Since the procedure ought to be designed for post-processing, this objective focuses on the application of adjustment solutions. As not every mobile mapping image has direct correspondences to the aerial images, another distinction can be made between different strategies to propagate an orientation update, e.g. featurebased or IMU-based. IMU-based updates require inertial sensor readings, feature-based approaches rely solely on images, i.e. visual odometry.

\section{Evaluation and verification of adjusted mobile mapping data}

Whereas the registration accuracy between mobile mapping and aerial data is an important measure, the actual impact on the improved accuracy needs to be evaluated in real world scenarios. The last objective investigates the position accuracy of the mobile mapping images after the registration with the aerial images and a data adjustment. Different test scenarios are to be investigated and evaluated with the support of surveyed ground control points. Certainly, the goal is to improve the accuracy of the mobile mapping data. Another task of the entire procedure is, however, to verify the accuracy of existing mobile mapping data products as well. In other words, does a registration with aerial images enable a cost-efficient alternative to surveyed ground control points to understand whether the original accuracy of acquired mobile mapping data is affected by GNSS-induced positioning issues?

\subsection{Structure of the thesis}

The outline of this thesis comprises the first considerations on how to register aerial with mobile mapping images, the development of the aerial nadir and aerial oblique pipeline as well as a chapter dedicated to the evaluation and the verification of obtained adjustment results. Figure 1.3 gives an overview on the structure of the thesis. 


\section{Chapter 1: Introduction}

\section{Chapter 2: Investigating different feature extraction} methods

Part 1: Low-level tie feature extraction

Part 2: Utilisation of orientation parameters

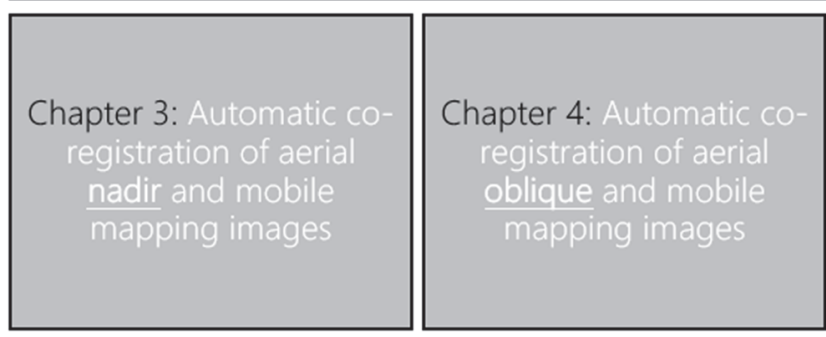

Chapter 5: Data adjustment and evaluation and verification of results

Chapter 6: Synthesis and outlook

Figure 1.3 Schematic overview of the thesis' chapters.

In particular, the thesis is structured in the following way:

Chapter 1 motivates the background for this work, links it to the current state of research, describes the research problem, and states the respective research objectives.

Chapter 2 is separated into two parts. The first part investigates various lowlevel tie feature extraction methods for the registration of mobile mapping and aerial (nadir) images. Moreover, first strategies to homogenise the data sets are described in this part, e.g. an inverse perspective method to increase the resemblance of the terrestrial and aerial data. The second part of this chapter examines multiple methods to increase the robustness of the registration problem in hand, in particular the utilisation of orientation parameters and the combination of corner features and template matching techniques.

Chapter 3 describes the development of a fully automatic pipeline for the registration of mobile mapping and aerial nadir images. Moreover, this chapter introduces an array of different techniques to increase the accuracy of the 
registration, such as an initial transformation and a potent correlation method, which can overcome the strong illumination differences between the data sets. Additionally, exemplary adjustment results are presented in this chapter to examine the achievable accuracy with this registration technique.

Chapter 4 covers the considerations and the development of the registration pipeline between mobile mapping and aerial oblique images. Although similar to the previous registration case with respect to diverging perspectives, a successful registration between mobile mapping and aerial oblique images requires an entirely different strategy. Based on a sparse point cloud derived from mobile mapping images, planes are estimated in object space for the identification of surfaces, which are suitable for the projection of the mobile mapping and aerial oblique image content. This step simplifies the registration considerably as resulting image patches can be registered with template matching techniques, which do not need to account for perspective distortions. Various parameter settings are highlighted and tested for robustness in the experiments section.

Chapter 5 summarises both registration pipelines briefly and explores their potential for the correction of mobile mapping data in the city of Rotterdam, which serves as our test area. In total, four different trajectories with different characteristics and 30 surveyed ground control points are used for this experiment. The adjustment procedure is designed to work with both, the nadir and oblique pipeline. The adjustment results are not only compared with respect to the different trajectories but also between the nadir and oblique aerial data, and a combination of both.

Chapter 6 concludes this dissertation with a synthesis of the results and an outlook.

It has to be mentioned that the chapters 2 to 5 are based on published journal or conference papers. Hence, some information may be repetitive or appear redundant. This may be, however, useful, as every chapter can be treated and regarded individually and does not require the reader to study previous chapters or the entire work to follow a chapter of interest. 


\section{Chapter 2 - Investigating different feature extraction methods ${ }^{13}$}

${ }^{13}$ Part 1 of this chapter is based on:

Jende, P., Hussnain, Z., Peter, M., Oude Elberink, S., Gerke, M., \& Vosselman, G. (2016) 'Low-level tie feature extraction of mobile mapping data (MLS / images) and aerial imagery', ISPRS Archives; Vol. XL-3/W4, https://doi.org/10.5194/isprs-archives-XL-3$\underline{\mathrm{W}} 4-19-2016$

Part 2 of this chapter is based on:

Jende, P., Peter, M., Gerke, M., \& Vosselman, G. (2016) 'Advanced tie feature matching for the registration of mobile mapping imaging data and aerial imagery', ISPRS Archives; Vol. XLI-B1, https://doi.org/10.5194/isprs-archives-XLI-B1-617-2016 


\subsection{Part 1 - Low-level tie feature extraction of mobile mapping data (MLS/images) and aerial imagery ${ }^{14}$}

\subsubsection{Abstract}

Mobile mapping is a technique to obtain geo-information using sensors mounted on a mobile platform or vehicle. The mobile platform's position is provided by the integration of Global Navigation Satellite Systems (GNSS) and Inertial Navigation Systems (INS). However, especially in urban areas, building structures can obstruct a direct line-of-sight between the GNSS receiver and navigation satellites resulting in an erroneous position estimation. Therefore, derived MM data products, such as laser point clouds or images, lack the expected positioning reliability and accuracy. This issue has been addressed by many researchers, whose aim to mitigate these effects mainly concentrates on utilising tertiary reference data. However, current approaches do not consider errors in height, cannot achieve sub-decimetre accuracy and are often not designed to work in a fully automatic fashion. We propose an automatic pipeline to rectify $M M$ data products by employing high resolution aerial nadir and oblique imagery as horizontal and vertical reference, respectively. By exploiting the MM platform's defective, and therefore imprecise but approximate orientation parameters, accurate feature matching techniques can be realised as a pre-processing step to minimise the MM platform's threedimensional positioning error. Subsequently, identified correspondences serve as constraints for an orientation update, which is conducted by an estimation or adjustment technique. Since not all MM systems employ laser scanners and imaging sensors simultaneously, and each system and data demands different approaches, two independent workflows are developed in parallel. Still under development, both workflows will be presented and preliminary results will be shown. The workflows comprise of three steps; feature extraction, feature matching and the orientation update. In this part of the chapter, initial results of low-level image and point cloud feature extraction methods will be discussed as well as an outline of the project and its framework will be given.

\subsubsection{Introduction}

Mobile mapping is on the verge of becoming a substantial addition to the family of geo-data acquisition techniques. Airborne or satellite data cover large areas, but have limited capabilities when it comes to the density of data postings and

\footnotetext{
${ }^{14}$ This is the only contribution in this work that has been co-authored by Zille Hussnain, whose research focused on the utilisation of aerial images for the correction of MLS trajectories. Although this is not the main topic of this research work regarding mobile mapping images, sections mainly authored by Zille Hussnain intentionally remain part of this contribution in order not to interrupt this chapter's coherence.
} 
high accuracy, whereas classical terrestrial techniques are expensive and often impractical. Particularly in urban areas, MM shapes up to be an extraordinarily useful technique not just to complement airborne or satellite coverage, but to enable a completely new array of possibilities. MM imaging systems and laser scanners collect high-resolution data, but have to rely on external georeferencing by GNSS. As GNSS being intermittently available, INS provides relative measures between position fixes and compensates for measurement noise and errors. Although GNSS carrier-phase measurements allow highly accurate positioning, urban areas remain problematic regarding the measurement reliability due to multipath effects and occlusions. When these phenomena persist over longer periods, accurate positioning cannot be maintained, and consequently data accuracy will be diminished (Godha et al., 2005).

This part presents a method to detect and extract low-level image and point cloud features as a prerequisite for the rectification of MM data using aerial imagery. First, a brief outline of the project will be given. In section 2, a literature overview on similar work will be presented, and applied feature detection and extraction methods will be shortly introduced, followed by section 4 addressing low-level feature extraction for images as well as for point clouds. Section 5 discusses initial results of low-level feature extraction methods of both aerial and MM images as well as point cloud data. Lastly, section 6 concludes the work presented in this part of the chapter as well as gives an outlook on future developments.

\subsubsection{Project Overview}

The aim of our research project is to enable a reliable localisation pipeline for MM data obtained in urban areas, and to verify existing data sets according to their localisation accuracy in order to economise the acquisition of ground control. Due to apparent differences in the sensor setup and data, two workflows for Mobile Laser Scanning (MLS) and Mobile Mapping Imaging (MMI) are being developed. The common basis is the utilisation of high-resolution aerial nadir and oblique imagery as an external reference to compensate for vertical as well as for horizontal errors. In a first stage, common features between the ground data and aerial nadir imagery are sought. Based on the imprecise, but approximate exterior orientation of the MM data, more reliable and efficient matching techniques can be employed. For instance, a confined search for correspondences and their verification in the other image can be inferred even from coarse orientation parameters. The next stage will be the integration of oblique images into the pipeline to yield common features on the vertical axis in order to better detect errors in height, and to increase the overall number of tie features considerably. Façades and other vertical objects, such as street lights and traffic signs, are potential objects which can be used 
for that purpose in the future. In a last step, this tie information allows for either a re-computation of the trajectory or, alternatively, an adjustment of the data as such.

\subsubsection{Related Work}

\subsubsection{Previous approaches}

Coping with poor localisation of mobile platforms in urban areas has been addressed by many authors. Mostly by employing tertiary data as an external reference, either the data itself (Jaud et al., 2013; Ji et al., 2015; Tournaire et al., 2006) or the platform's trajectory (Kümmerle et al., 2011; Leung et al., 2008; Levinson and Thrun, 2007) has been corrected. Depending on the data input and type (e.g. aerial imagery, digital maps or ground control points), different registration methods were utilised to impose unaffected, reliable and precise orientation information from external data on MM data sets. Subsequently, yielded correspondences were used as a constraint within a filter or adjustment solution. Even though many authors achieved a successful localisation based on an external reference, errors in height were not corrected, and a consistent sub-decimetre accuracy could not be reached.

\subsubsection{Low-level Feature Extraction}

Both, low- and high-level feature extraction methods, are relevant for this research project. Whereas low-level features allow a great flexibility towards the selection of suitable correspondences, the registration of data originating from different sensors (i.e. Mobile Laser Scanning and aerial imagery) may demand an extension of that concept. Although MLS intensity information enables the derivation of corner features, an abstract representation by identifying common objects in both data sets can facilitate determining thorough and reliable transformation parameters. Hence, high-level feature extraction methods will be highlighted in the future. In this part of the chapter, however, emphasis will be placed on low-level feature extraction which is still an active field of research as real-time applications have been gaining more attention in the last few years. Classic feature detection algorithms, such as the Förstner-Operator (Förstner and Gülch, 1987) or the Harris Corner Detector (Harris and Stephens, 1988) are accompanied by state-of-the-art approaches like AKAZE (Alcanterilla et al., 2013) or FAST (Rosten and Drummond, 2006). Although many improvements have been made in this field, the most important property of a feature detector remains to identify the same keypoints over a set of images.

Once features have been detected in the image, they have to be described unambiguously to increase their distinctiveness among other features in order to match them correctly. Low-level feature description approaches can be 
divided into two categories - binary and float description. Whereas float descriptors, such as SIFT (Lowe, 2004), are based on a Histogram of Oriented Gradients (HoG), binary descriptors (e.g. BRIEF, Calonder et al. (2010)) are analysing the neighbourhood of a feature keypoint with a binary comparison of intensities according to a specific sampling pattern. Float descriptors are typically more expensive to compute, and need more memory to store their output than binary descriptors. However, depending on the application, robustness of these two categories varies (Heinly et al., 2012; Miksik and Mikolajczyk, 2012). In this part of the chapter, different feature detection as well as float and binary description methods will be compared taking the example of aerial nadir, MM panoramic imagery and intensity images derived from MLS data. Feature keypoints across the data sets will be computed with SIFT, KAZE (Alcantarilla et al., 2012), AKAZE and the Förstner Operator.

SIFT detects blobs with a Difference-of-Gaussian method at different scaled instances of the image. KAZE computes a non-linear scale space using an additive operator splitting technique, where keypoints are detected at locations with a maximum response of the determinant of the Hessian matrix. Similarly, AKAZE also relies on keypoint detection based on the Hessian matrix, but computes a non-linear scale space with fast explicit diffusion. Förstner detects corners based on the search for local minima of eigenvalues of a covariance matrix of image gradients. Except for Förstner, all aforementioned procedures allow for an additional feature description. SIFT utilises a HoG in a local neighbourhood to describe a keypoint. KAZE's keypoints are described with the SURF descriptor (Bay et al., 2008) modified to be compatible with the detector's non-linear scale space. AKAZE uses a binary description based on an adapted version of Local Difference Binary (Yang and Cheng, 2012) where sample patches around the keypoint are averaged and then compared in a binary manner. For Förstner keypoints, LATCH (Levi and Hassner, 2015) has been used for a binary feature description. LATCH compares sample-triplets around a keypoint, where the sampling arrangement is learnt. Respective results will be discussed 2.1.6.

\subsubsection{Low-Level Tie Feature Extraction}

\subsubsection{MMI \& Aerial Nadir Images}

Aerial nadir ortho-images with a ground sampling distance of approximately 12 centimetres serve as the reference data set in this project. The MM images are 360*180 degrees panoramic images (Figure 2.1) acquired every 5 metres along the platform's trajectory. For more details and specifications, please see Beers (2011). In order to use the aerial images' exterior orientation for the rectification of MM data successfully, respective tie information has to be reliable and accurate. Although ground and aerial nadir data have a different perspective on the scene, low-level feature correspondences can be identified 
in all data sets. For example, corners of road markings, centres of manholes and building corners resemble each other across all sensors.

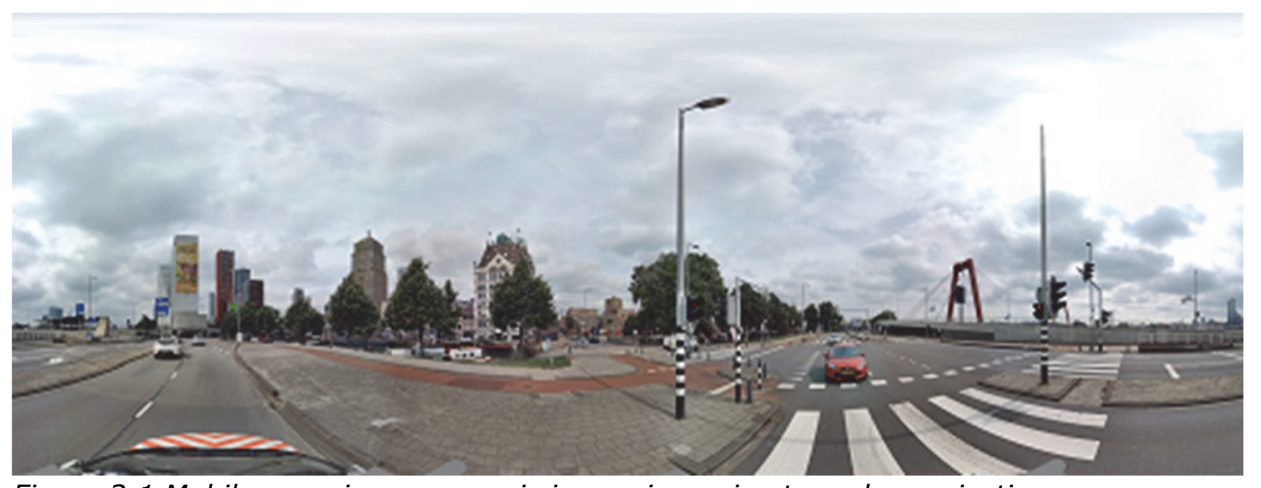

Figure 2.1 Mobile mapping panoramic image in equirectangular projection.

\section{Pre-Processing}

In order to simplify and optimise feature matching, the panoramic images are projected onto an artificial ground plane to increase the resemblance to the aerial images. The ground plane is computed based on the location of the MM imaging sensor and the fixed height of the sensor above ground. Especially in areas where the actual ground is not exactly flat, this approximation can lead to certain distortions (see Figure 2). In the future, the rather reliable relative orientation between two recording locations will be used to compute a more accurate plane. Since this part of the chapter focuses solely on feature detection and description, and the aerial images used are ortho-projected, this fact can be neglected for now.

MM panoramic images are stored in an equirectangular projection, encoding directly spherical coordinates for every image pixel. Therefore, no projection matrix or other intrinsic parameters are needed to reproject the panoramic image. The quadratic ground plane is centred at the dropped perpendicular foot of the respective recording location. Analogue to the aerial imagery's resolution of 12 centimetres, the ground plane is rasterised holding a world coordinate for every cell. Subsequently, each raster cell's coordinate is backprojected into the panoramic image in order to extract the respective RGB value, and transfer the information back onto the ground plane.

Since every back-projected ray will pierce the image plane of the panoramic image, and thus every raster cell will contain an RGB value, an interpolation of the resulting projected image seems dispensable. However, the geometric representation of the pixels of both grids varies, leading to multiple assignments of the same RGB value especially at the edge of the projected image appearing as blur. Hence, a bilinear interpolation of the extracted value according to the pixel neighbourhood of the panoramic image is conducted. 
Consequently, every pixel in the projected image is composed of an individual set of grey values.

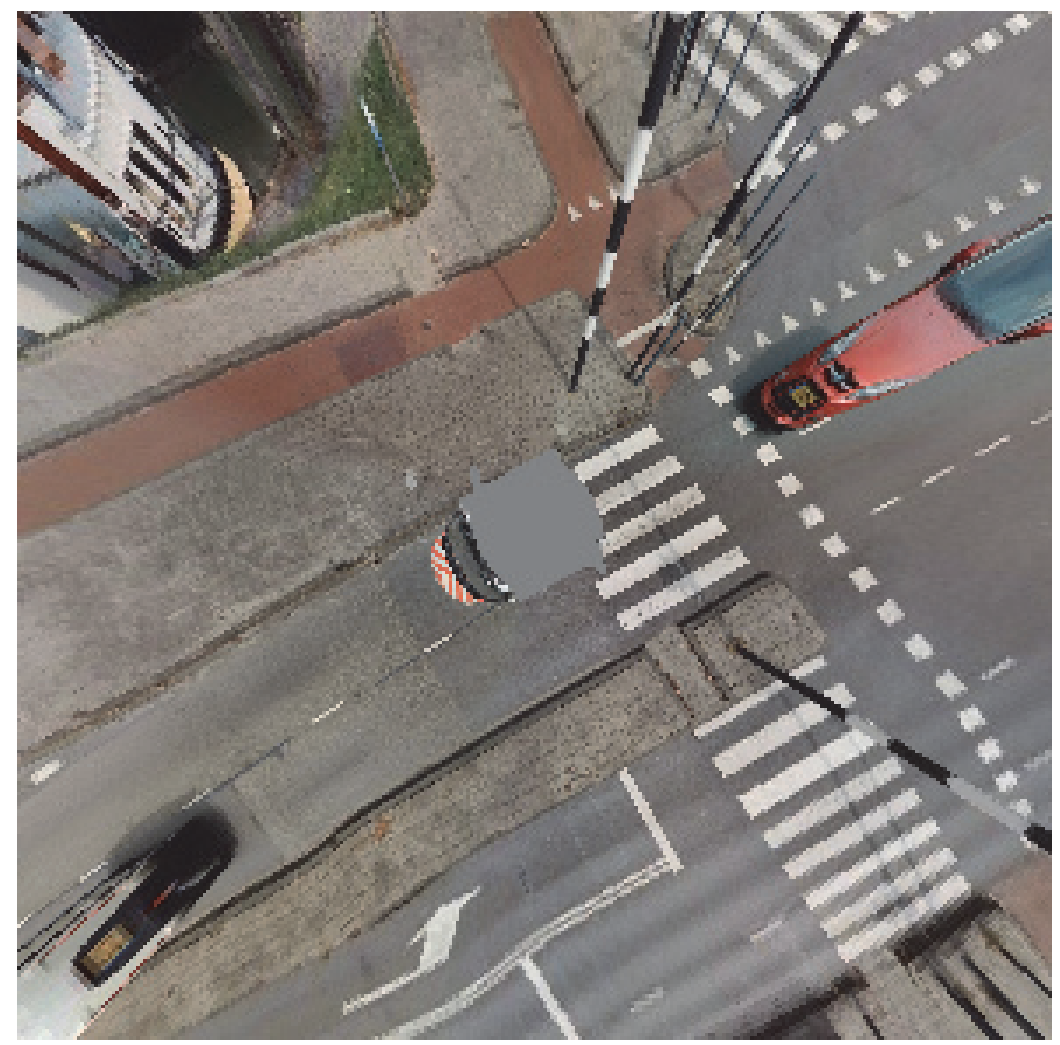

Figure 2.2 Panoramic image projected onto an artificial ground plane.

\section{Feature Extraction}

The only overlapping area for feature detection induced by different original perspectives between aerial ortho-images and MM images is the road surface and its immediate vicinity. Therefore, road markings, such as zebra crossings or centre lines are being targeted on for feature detection. Resulting from atmospheric conditions and motion blur (esp. cameras without forward motion compensation), the image quality of the aerial photographs can be affected. To compensate for these effects, the projected panoramic images might need to be blurred even though sharing the same resolution with the aerial image. In the process of projecting the panoramic images onto the ground, not just the projection but also the approximate scale and rotation of the aerial image have been retrieved simultaneously. In particular, this circumstance simplifies the matching process considerably, but also renders to be useful for the step of feature description as less invariances and therefore fewer ambiguities have to be considered by the descriptor; i.e. the descriptor does not have to account 
for scale and rotational invariance since the panoramic image is north oriented and has got the same resolution.

On the other hand, the images have not been acquired at the same time and with different sensor systems. Consequently, this fact is resulting in another category of a description problem. For instance, changes in illumination and contrast may affect the computation of the descriptor.

Moreover, repetitive patterns of road markings (e.g. zebra crossings) cannot be ignored as they may result in false feature matches. Either this issue has to be tackled on the descriptor level or during the matching stage. Introducing rules, such as ordering constraints (Egels and Kasser (2001), p. 198)or perceptual grouping (Lowe (1985), p.4), to describe a chain or group of adjacent features may prevent misassignment. Additionally, approximate camera parameters can be exploited within the matching procedure. By backprojecting identified keypoints into the other image, a window can be defined to constrain the search for correspondences. These methods are currently under development or labelled future work. Aforementioned feature detection and description procedures will be applied to our data sets and results will be discussed 2.1.6.

\subsubsection{Mobile Laser Scanning}

The Mobile laser scanning point cloud (MLSPC) is acquired from one or more lidar sensors mounted on a moving car. The car's trajectory is estimated by GNSS and IMU, where a GNSS based position is retrieved after one-second intervals. The IMU is used to interpolate all intermediate positions. A particular mobile mapping car moving at a speed of $36 \mathrm{~km} / \mathrm{h}$ covers an area of $10 \mathrm{~m}$ in 1 second. During this one-second interval, the IMU provides relatively accurate positions, which favours to crop MLCPC patch-wise, where the size of each patch is 10 by $10 \mathrm{~m}$. State of the art laser scanning systems claim to achieve a relative accuracy of $10 \mathrm{~mm}$ when a control point is provided within $100 \mathrm{~m}$ of scanning. Thus, even if the scanning is conducted at a slower acquisition speed, the 10 by $10 \mathrm{~m}$ patch would not be affected by (IMU-based) distortions to an extent that would hamper feature extraction. Moreover, the point cloud which has been used in this project, already has an absolute accuracy in sub-metre range for roughly $25 \mathrm{~km}$ of scanning, which means that the relative accuracy of the point cloud is still within a $10 \mathrm{~mm}$ range.

Thereafter, each cropped point cloud patch is converted to an ortho-image by assigning a barycentric interpolation of laser intensities to its corresponding image pixel. A particular point cloud patch and the generated ortho-image is shown in Figure 2.3. 
The proposed method detects low-level features from ortho-image gradients using SIFT, KAZE, AKAZE, and the Förstner detector. The feature point description is obtained from SIFT, KAZE, AKAZE and LATCH.

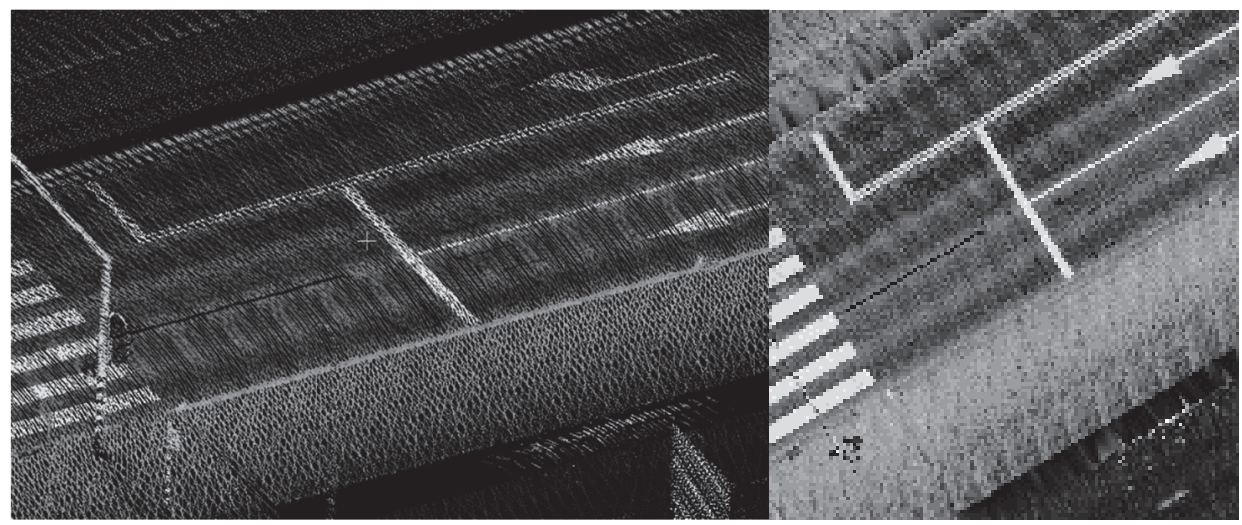

Figure 2.3 Point cloud patch (left) to ortho-image conversion (right).

\subsubsection{Results}

In this section, feature detection and description methods will be compared according to their potential for deriving significant tie features and correspondences between aerial nadir and mobile mapping panoramic images as well as between aerial nadir and MLS intensity images. First, a comparison between SIFT ${ }^{15}$, KAZE, AKAZE and Förstner ${ }^{16}$ on each of the three data sets will be conducted. Subsequently, acquired keypoints will be described with their corresponding method except for Förstner where a LATCH description will be used. Although still under development, feature matching will be utilised to compare the quality of each descriptor. To this end, simple descriptor matching to yield correspondences and a homography estimation to detect outliers will be used. As the focus of this project is on urban areas, four subsets with each $15 \mathrm{~m}$ side length of a typical road scene between two intersections have been selected for this experiment (Figure 2.4).

\footnotetext{
${ }^{15}$ For SIFT, KAZE, AKAZE, and LATCH, their respective OpenCV implementation has been used.

${ }^{16}$ Implementation of the Förstner operator by Marc Luxen, University of Bonn.
} 


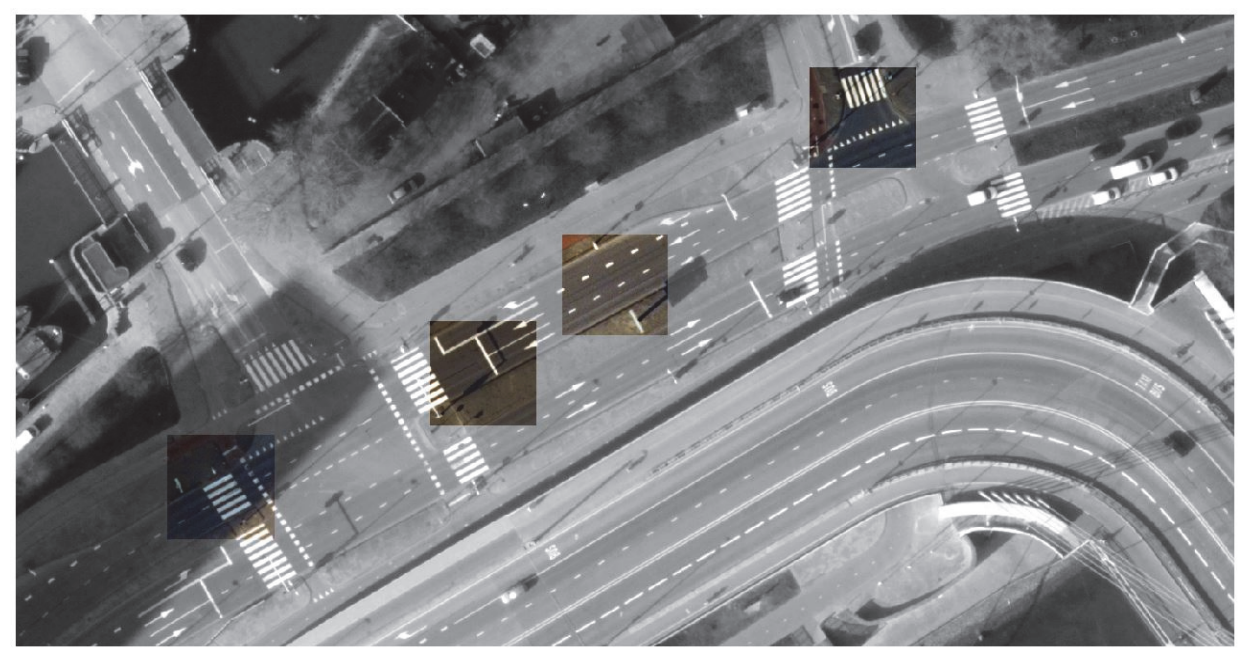

Figure 2.4 Four subsets of a typical urban scene (coloured tiles from scene 1 on the left to scene 4 on the right).

\subsubsection{Feature detection}

In urban areas, road markings and other prominent objects, such as kerbstones or manholes, identifiable among all data sets are favoured for feature detection. However, due to noise and different original perspectives, it is considered a challenging task for the step of feature detection to maintain a comparable detection rate over the entire data set.

Depending on the scene, this detection rate varies. The number of road markings and the detector itself highly influence the results. For instance, due to its scale invariance SIFT detects keypoints on different blurred instances of the same image, and thus yields a lot more potential features than a corner detector, such as Förstner. As it will be shown in section 2.1.6.2, a potent feature detection alone is not sufficient for a successful registration.

Table 2-1 Number of combined keypoints over all subsets per detection method.

\begin{tabular}{lcccc}
\hline & Aerial Image & $\begin{array}{c}\text { Panoramic } \\
\text { Image }\end{array}$ & MLS Intensity & Total \\
\hline \hline SIFT & 234 & 379 & 810 & 1423 \\
KAZE & 119 & 304 & 458 & 881 \\
AKAZE & 29 & 68 & 175 & 272 \\
Förstner & 40 & 75 & 153 & 268 \\
\hline
\end{tabular}

\section{SIFT}

SIFT yields more keypoints than any other method used in the experiments in this part of the chapter (Table 2-1). It detects $60 \%$ keypoints more than KAZE and even 5 times more keypoints than AKAZE or Förstner. Being very sensitive to image noise and detecting keypoints on different image scales, the detected 
features are not always useful. In particular, this comes into effect for both types of MM images as they have a higher original resolution and therefore a higher entropy (Figure 2.5).

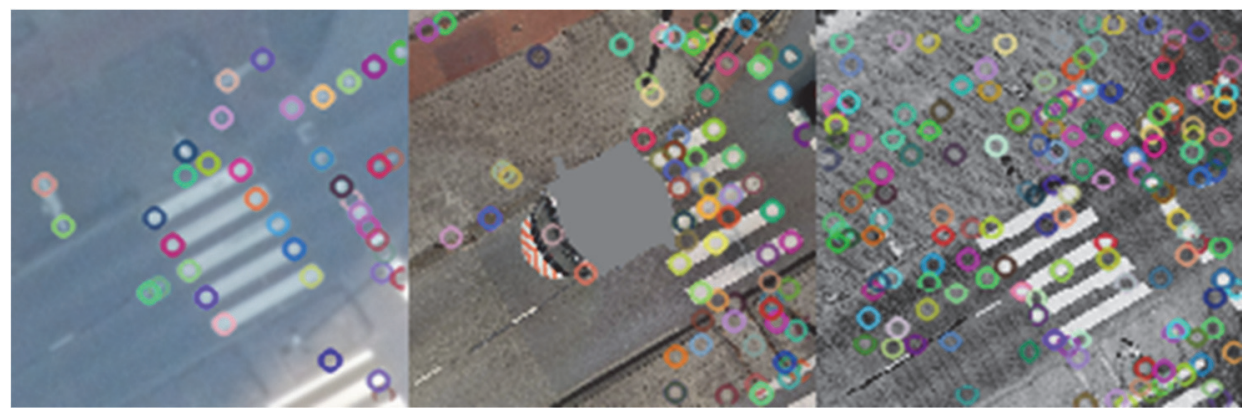

Figure 2.5 SIFT keypoints detected in aerial image (left), panoramic image (centre) and MLS intensity image (right).

\section{KAZE}

KAZE detects fewer keypoints than SIFT, but still considerably more than AKAZE or Förstner. However, road markings are very well preserved, and especially their corner features, which are the most important image entity in our case, were mostly detected (Figure 2.6).

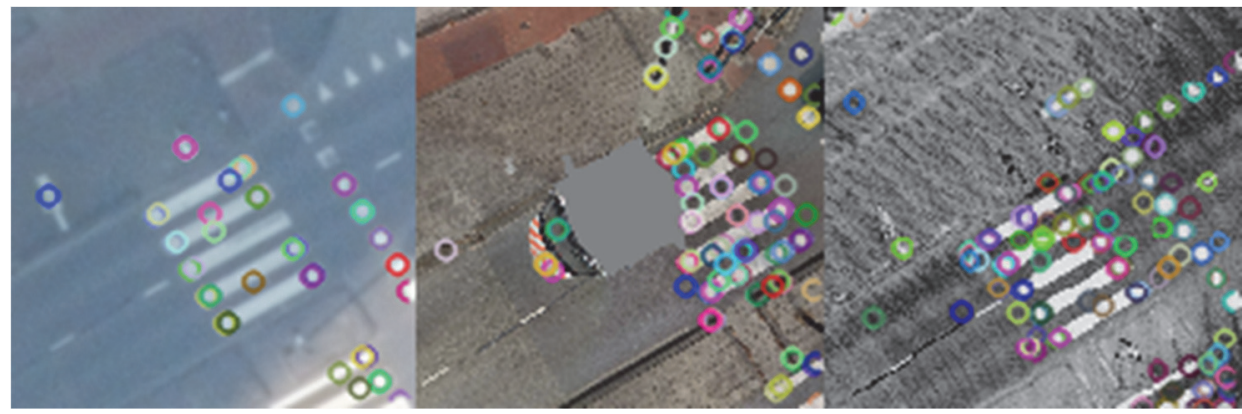

Figure 2.6 KAZE keypoints detected in aerial image (left), panoramic image (centre) and MLS intensity image (right).

\section{AKAZE}

Although, AKAZE and KAZE are quite similar in the way how a feature is detected (determinant of Hessian), their main difference lies in the computation of image pyramids to detect keypoints at different image scales. AKAZE detects fewer keypoints than KAZE, but these keypoints are most often important corners of road markings (Figure 2.7). Nonetheless, in two of four aerial images, AKAZE only detected one single keypoint which turns out to be too few for matching purposes. 


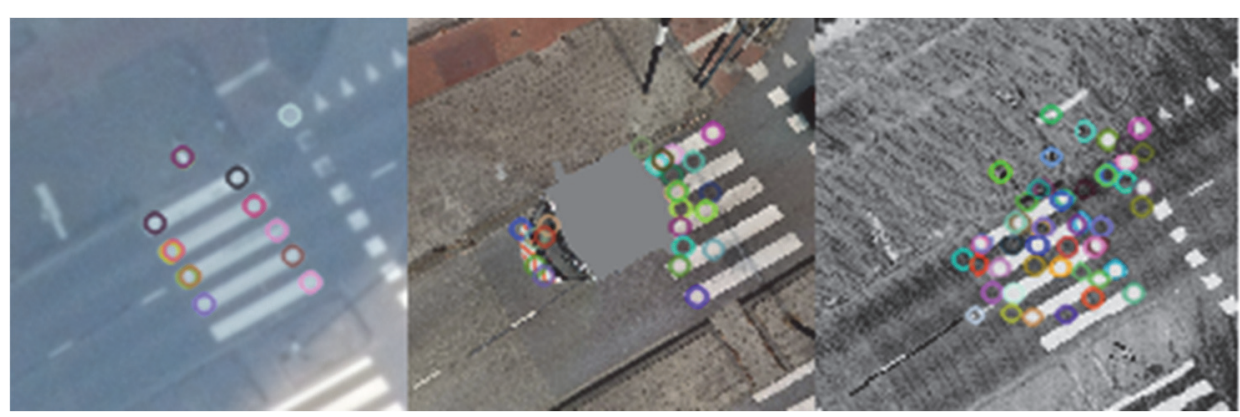

Figure 2.7 AKAZE keypoints detected in aerial image (left), panoramic image (centre) and MLS intensity image (right).

\section{Förstner Operator}

The Förstner Operator is the only feature detector without the consideration of scale. This, and the fact that Förstner detects features solely at corners and centres of small image objects, leads to a very deliberate keypoint detection. However, almost every detected feature can be regarded as significant for the registration process. Due to its capability of sub-pixel localisation of keypoints, the same object point can be represented slightly shifted among different image sources which shapes up as a challenge for feature description (Figure 2.8).

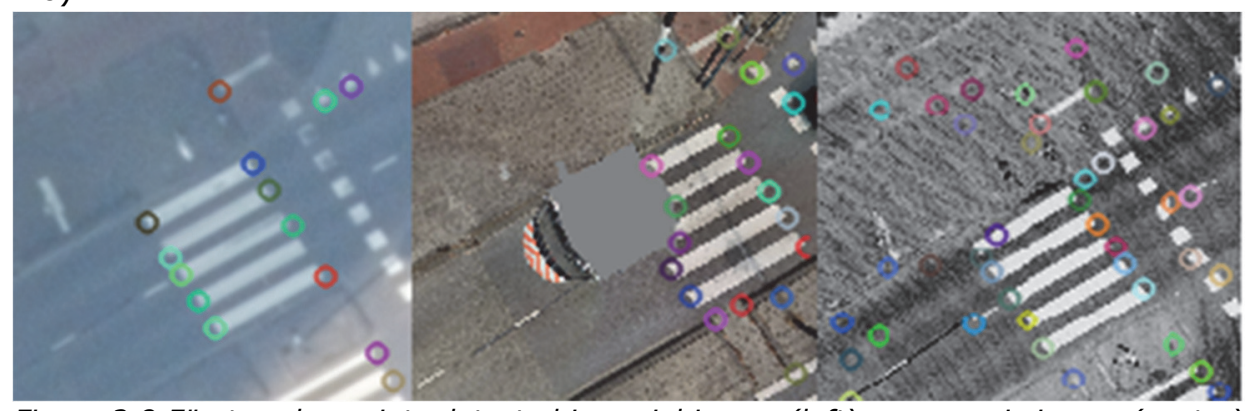

Figure 2.8 Förstner keypoints detected in aerial image (left), panoramic image (centre) and MLS intensity image (right).

\subsubsection{Feature description}

Identified keypoints need to be described unambiguously to enable feature matching between two images. In general, difficulties arise if there is a change in perspective, illumination, coverage, or scale between the images as well as ambiguities resulting from repetitive patterns. In order to obviate apparent difficulties for registering the images, the MM data has been projected onto the ground to increase the resemblance to aerial imagery. As a consequence, scale and perspective are more similar among the data sets, but differences in illumination and coverage cannot be mitigated easily. Thus, different description methods are evaluated with regard to their ability to cope not just with the aforementioned changes but also to their performance to bridge sensor-induced differences. 
SIFT, KAZE, AKAZE and LATCH will be used for feature description. As mentioned earlier, LATCH will be used for keypoints detected with the FörstnerOperator. To measure the descriptor quality of each method, the images have to be matched. The number of matches, inliers classified by RANSAC as well as the actual number of correct correspondences will be compared among different descriptors. Two out of four scenes (scene 1 and scene 2) will be discussed in detail3. Moreover, for every test scene, four different settings have been tested. To this end, MM data has been blurred with a Gaussian filter to increase the resemblance to the aerial data set. Moreover, a resampling of all data sets has been conducted as it has been shown that increasing the sampling size can facilitate a feature's distinctiveness considerably (Köthe, 2003b).

$1^{\text {st }}$ run No modification of source images

$2^{\text {nd }}$ run Gaussian blurring of source images

$3^{\text {rd }}$ run Resampling to $150 \%$ of original size

$4^{\text {th }}$ run Blurring and subsequent resampling of source images

\section{Aerial images and panoramic images}

\section{Scene 1}

The first scene comprises of a zebra crossing and dotted road markings aggravating correct matching due to possible descriptor ambiguity. If enough correct correspondences are found, RANSAC converges to a correct solution. In the first run, however, none of the methods was able to achieve a good result (see e.g. Figure 2.9). The derived keypoint descriptors were not distinct enough to be matched accordingly. By blurring the images with a Gaussian low-pass filter in the second iteration, results slightly improved for KAZE (see Table 2-2). Yet, by resampling the source images to $150 \%$ of their original size, results got significantly better especially for KAZE, but also a bit for SIFT and AKAZE (see Figure 2.10). In the fourth run, a Gaussian blurring followed by a resampling did not have an impact on the matching quality of this scene (see Table 2-3). Furthermore, LATCH yielded very poor results regardless of the iteration. 


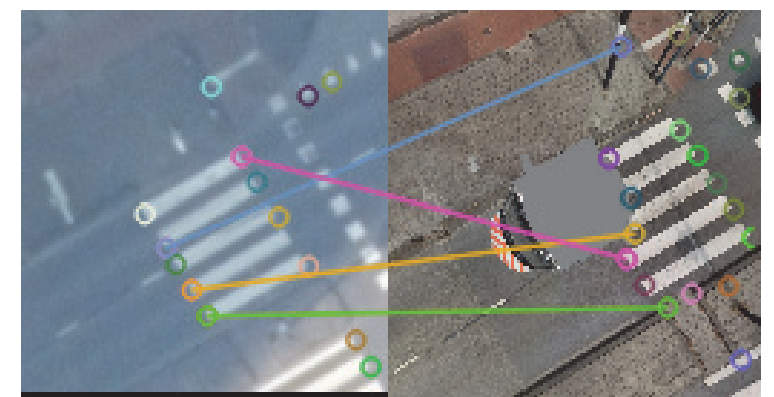

Figure 2.9 Matched LATCH keypoints in the first scene and first iteration.

Table 2-2 Matching results of scene 1 between aerial and panoramic image of the 1st and 2 nd iteration.

\begin{tabular}{lccc|ccc}
\hline & \multicolumn{3}{c|}{$1^{\text {st }}$ run } & \multicolumn{3}{c}{$2^{\text {nd }}$ run } \\
& Matches & Inliers & $\begin{array}{c}\text { Correct } \\
\text { Matches }\end{array}$ & Matches & Inliers & $\begin{array}{c}\text { Correct } \\
\text { Matches }\end{array}$ \\
\hline \hline SIFT & 61 & 15 & 1 & 61 & 13 & 0 \\
KAZE & 41 & 12 & 1 & 41 & 12 & 2 \\
AKAZE & 14 & 6 & 0 & 14 & 6 & 0 \\
LATCH & 9 & 4 & 0 & 9 & 0 & 0 \\
\hline
\end{tabular}




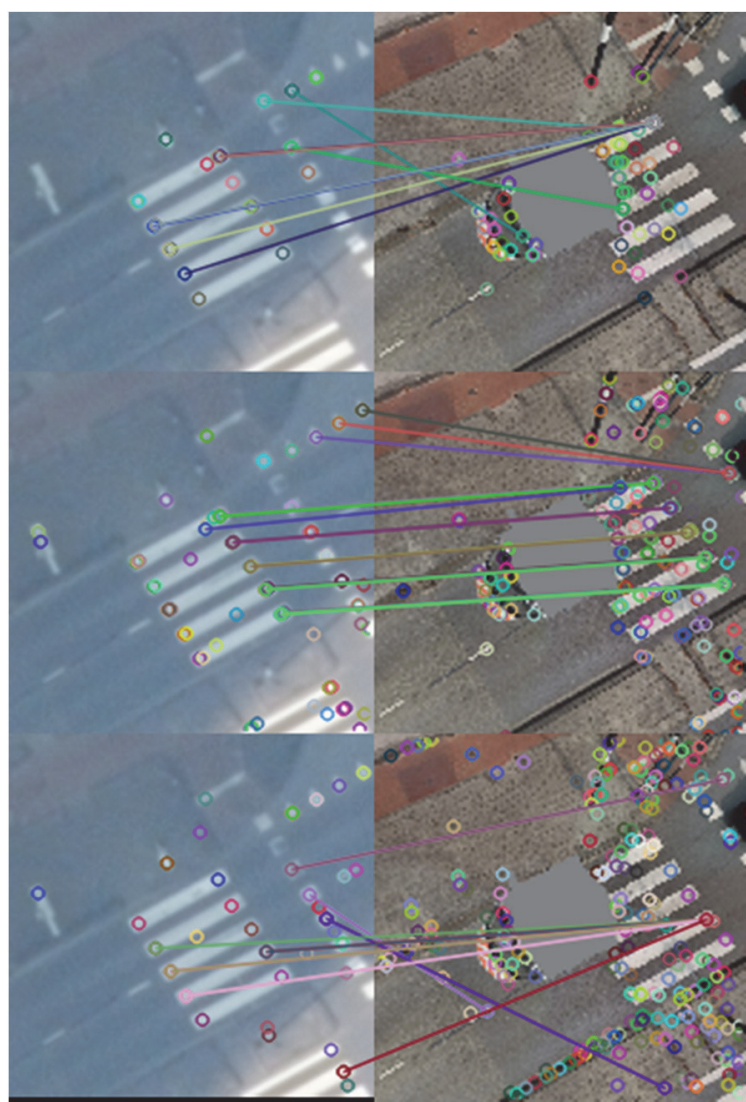

Figure 2.10 Comparison of matching results of AKAZE (top), KAZE (centre) and SIFT (bottom) in 3rd run of the 1st scene.

Table 2-3 Matching results of scene 1 between aerial and panoramic image of the 3rd and 4th iteration.

\begin{tabular}{lccc|ccc}
\hline & & \multicolumn{3}{c|}{$1^{\text {st }}$ run } & \multicolumn{3}{c}{$2^{\text {nd }}$ run } \\
& Matches & Inliers & $\begin{array}{c}\text { Correct } \\
\text { Matches }\end{array}$ & Matches & Inliers & $\begin{array}{c}\text { Correct } \\
\text { Matches }\end{array}$ \\
\hline \hline SIFT & 59 & 15 & 2 & 59 & 16 & 0 \\
KAZE & 78 & 17 & 10 & 78 & 16 & 10 \\
AKAZE & 24 & 9 & 2 & 24 & 9 & 0 \\
LATCH & 7 & 4 & 0 & 7 & 0 & 0 \\
\hline
\end{tabular}

\section{Scene 2}

The second scene shows linear road markings and parts of a zebra crossing. Whereas major parts of the zebra crossing and the dotted road markings were visible in the first scene, large parts of the road markings are covered by the mobile mapping car itself in the second scene which may impede the matching 
process. Similar to the first scene, unmodified imagery was difficult to match and LATCH nor AKAZE found a single correspondence. With SIFT, however, a couple of keypoints could be matched, even though just one correct correspondence has been identified (see Figure 2.11).

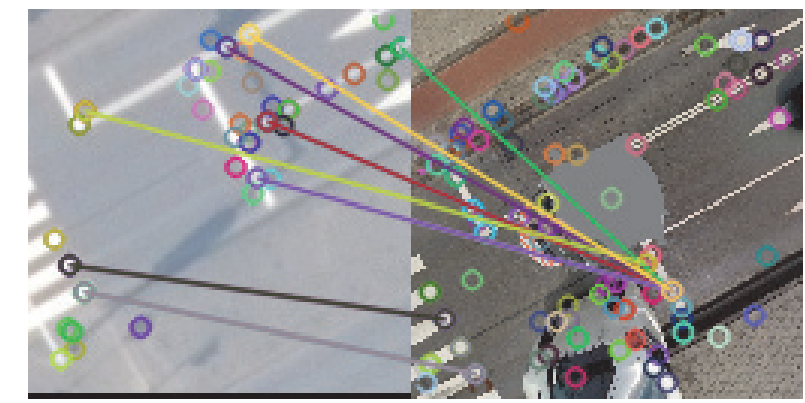

Figure 2.11 Matched SIFT keypoints in the second scene and first iteration (correct correspondence is light purple).

Table 2-4 Matching results of scene 2 between aerial and panoramic image of the 1st and 2 nd iteration.

\begin{tabular}{lccc|ccc}
\hline & & \multicolumn{3}{c|}{$1^{\text {st }}$ run } & \multicolumn{3}{c}{$2^{\text {nd }}$ run } \\
& Matches & Inliers & $\begin{array}{c}\text { Correct } \\
\text { Matches }\end{array}$ & Matches & Inliers & $\begin{array}{c}\text { Correct } \\
\text { Matches }\end{array}$ \\
\hline \hline SIFT & 59 & 15 & 2 & 59 & 16 & 0 \\
KAZE & 78 & 17 & 10 & 78 & 16 & 10 \\
AKAZE & 24 & 9 & 2 & 24 & 9 & 0 \\
LATCH & 7 & 4 & 0 & 7 & 0 & 0 \\
\hline
\end{tabular}

By blurring the images with a Gaussian filter, especially SIFT returns a better result. Albeit only two correct correspondences have been identified, results got considerably better (see Figure 2.12). Apparently, RANSAC removed a couple of outliers, and was able to stabilise the estimation of the homography. Without ground truth, the matched bars of the zebra crossing might appear as correct correspondences. These descriptor ambiguities have to be tackled on another processing level.

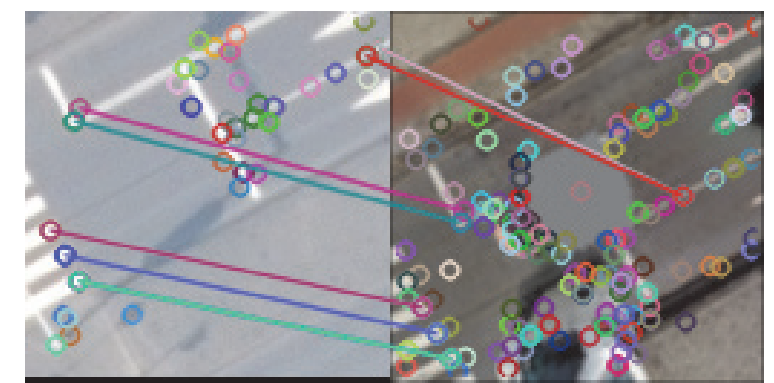

Figure 2.12 Matched SIFT keypoints in the second scene and second iteration. 
By resampling the images to $150 \%$ of their original size in the 3rd iteration, KAZE benefitted the most, although yielding only 4 correct matches out of 14 matches classified as inliers (see Figure 2.13).

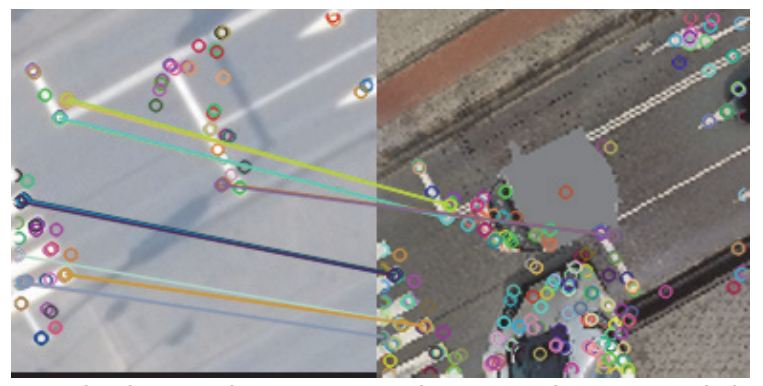

Figure 2.13 Matched KAZE keypoints in the second scene and third iteration.

Blurring the images prior to resampling them further improves the results for KAZE and AKAZE while decreasing the quality of SIFT's output (see Figure 2.14). Again, LATCH did not show any improvement.

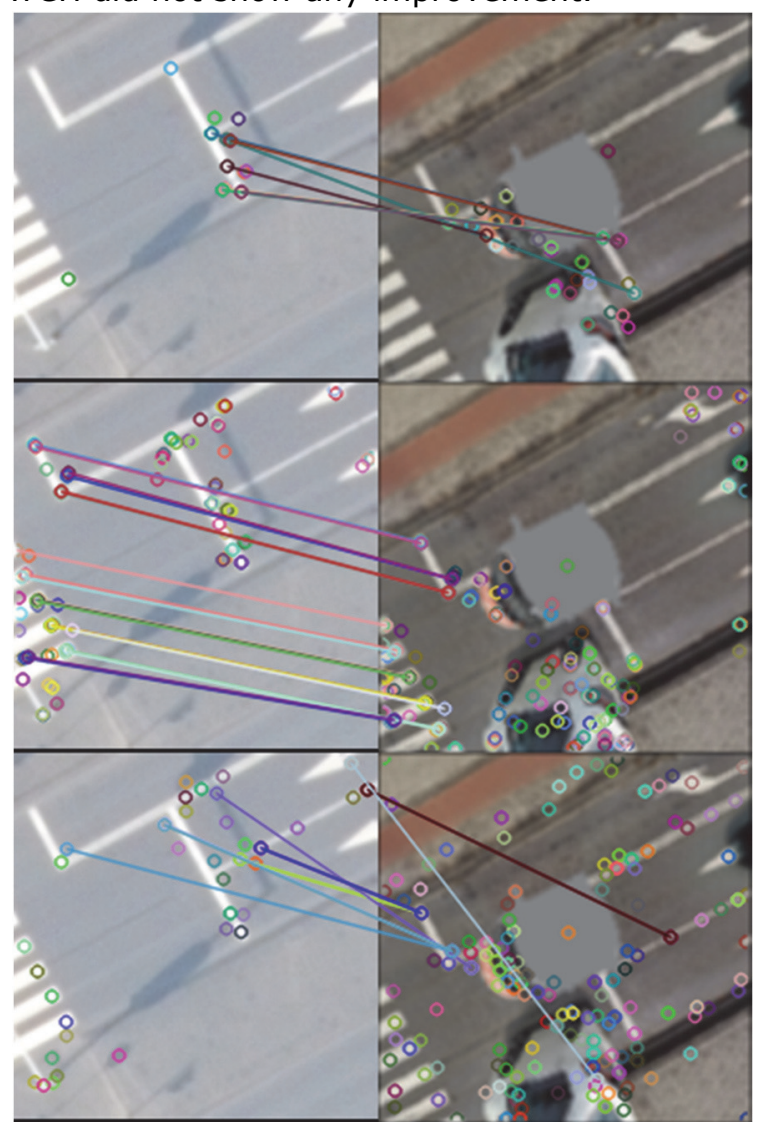

Figure 2.14 Comparison of matching results of AKAZE (top), KAZE (centre) and SIFT (bottom) in 4th run of the 2nd scene. 
Table 2-5 Matching results of scene 2 between aerial and panoramic image of the 3rd and 4th iteration.

\begin{tabular}{lccc|ccc} 
& \multicolumn{3}{c}{$3^{\text {rd }}$ run } & \multicolumn{3}{c}{$4^{\text {th }}$ run } \\
& Matches & Inliers & $\begin{array}{c}\text { Correct } \\
\text { Matches }\end{array}$ & Matches & Inliers & $\begin{array}{c}\text { Correct } \\
\text { Matches }\end{array}$ \\
\hline \hline SIFT & 51 & 12 & 0 & 51 & 11 & 1 \\
KAZE & 79 & 14 & 4 & 79 & 20 & 6 \\
AKAZE & 15 & 6 & 0 & 15 & 8 & 2 \\
LATCH & 3 & 0 & 0 & 3 & 0 & 0 \\
\hline
\end{tabular}

\section{Aerial images and MLS intensity images}

\section{Scene 1}

In the first run (Table 6), KAZE yielded the best results with only few mismatches, and those occurred due to descriptor ambiguity. AKAZE returns competitive results, however obtained fewer matches than KAZE. SIFT and LATCH both equally failed to achieve a reliable number of matches. In the second run, blurring the images dramatically improved the number of correct matches from SIFT descriptor. Similarly, KAZE's result also improved moderately. AKAZE has performed consistently and results did not improve. The image blurring did not have an effect on the poor results of the LATCH descriptor. In the third run (Table 7 ), resizing the images to $150 \%$, results of SIFT and KAZE have improved. Interestingly, all calculated matches are correct and there is no mismatch. The results from AKAZE have improved slightly as well. The fourth run - blurring and resizing the images - increased the number of inliers from KAZE, while there is no mismatch as shown in Figure 2.15. The total number of matches even decreased in case of AKAZE. 


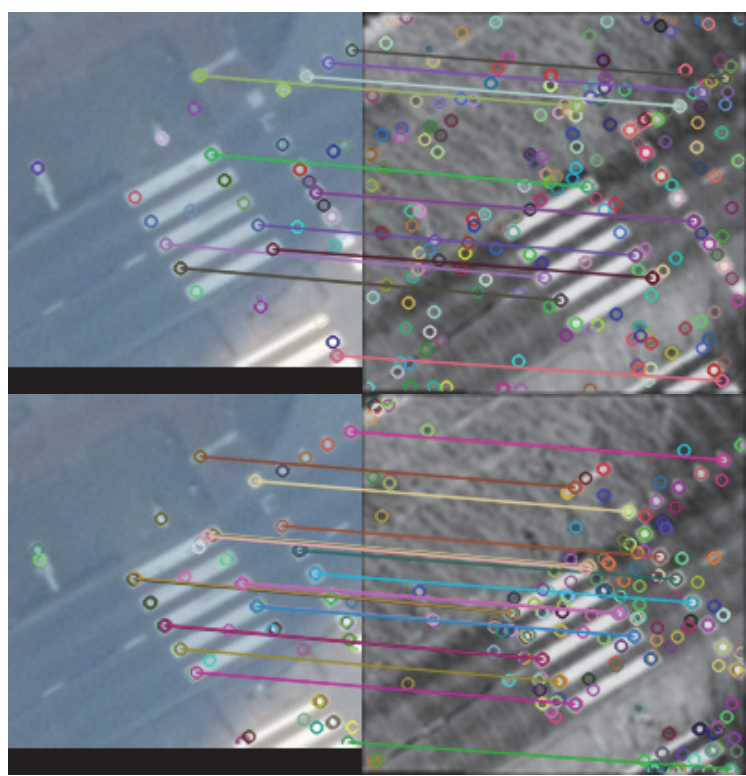

Figure 2.15 Comparison of SIFT (top) and KAZE (bottom) in 4th run on 1st scene.

Table 2-6 Matching results of scene 1 between aerial and MLS ortho-image of the 1st and 2 nd iteration.

\begin{tabular}{lccc|ccc}
\hline & & \multicolumn{3}{c|}{$1^{\text {st }}$ run } & \multicolumn{3}{c}{$2^{\text {nd }}$ run } \\
& Matches & Inliers & $\begin{array}{c}\text { Correct } \\
\text { Matches }\end{array}$ & Matches & Inliers & $\begin{array}{c}\text { Correct } \\
\text { Matches }\end{array}$ \\
\hline \hline SIFT & 61 & 15 & 1 & 61 & 14 & 12 \\
KAZE & 41 & 12 & 8 & 41 & 13 & 12 \\
AKAZE & 14 & 7 & 5 & 14 & 7 & 4 \\
LATCH & 9 & 4 & 0 & 9 & 4 & 0 \\
\hline
\end{tabular}

Table 2-7 Matching results of scene 1 between aerial and MLS ortho-image of the 3rd and 4th iteration.

\begin{tabular}{lccc|ccc}
\hline & \multicolumn{3}{c|}{$3^{\text {rd }}$ run } & \multicolumn{3}{c}{$4^{\text {th }}$ run } \\
& Matches & Inliers & $\begin{array}{c}\text { Correct } \\
\text { Matches }\end{array}$ & Matches & Inliers & $\begin{array}{c}\text { Correct } \\
\text { Matches }\end{array}$ \\
\hline \hline SIFT & 59 & 14 & 14 & 59 & 14 & 14 \\
KAZE & 78 & 19 & 19 & 78 & 20 & 20 \\
AKAZE & 24 & 9 & 6 & 24 & 8 & 3 \\
LATCH & 7 & 4 & 0 & 7 & 0 & 0 \\
\hline
\end{tabular}

\section{Scene 2}

In the first run (Table 2-8), on this difficult scene, all descriptors totally failed except KAZE, which also performed poorly due to descriptor ambiguity. SIFT 
also seemed to struggle with the descriptor ambiguity and therefore yielded no match. Blurring the images did not change anything, except that KAZE's result slightly improved. The third run (Table 2-9) did not lead to any significant improvements. In contrast to the first scene, the total number of matches from KAZE even decreased due to descriptor ambiguity. Although AKAZE was able to derive some matches, it cannot be considered a significant improvement. SIFT and LATCH failed to achieve a single match in the third run as well. In the fourth run, the number of matches from KAZE improved significantly. Interestingly, not a single mismatch could be identified. The respective results are shown in Figure 2.16. The SIFT results, however, did not improve and only a single match was returned.

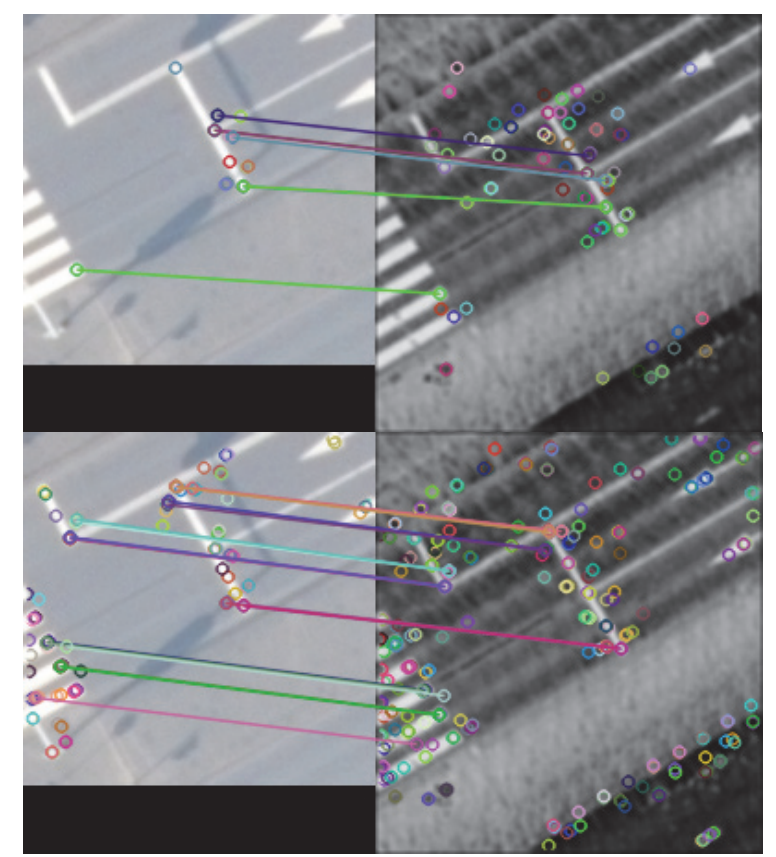

Figure 2.16 Matching results of AKAZE (top) and KAZE (bottom) in 4th run on scene 2.

Table 2-8 Matching results of scene 2 between aerial and MLS ortho-image of the 1st and 2nd iteration

\begin{tabular}{lccc|ccc} 
& \multicolumn{3}{c|}{$1^{\text {st }}$ run } & \multicolumn{3}{c}{$2^{\text {nd }}$ run } \\
& Matches & Inliers & $\begin{array}{c}\text { Correct } \\
\text { Matches }\end{array}$ & Matches & Inliers & $\begin{array}{c}\text { Correct } \\
\text { Matches }\end{array}$ \\
\hline \hline SIFT & 54 & 10 & 0 & 54 & 11 & 0 \\
KAZE & 42 & 10 & 4 & 42 & 11 & 7 \\
AKAZE & 1 & 0 & 0 & 1 & 0 & 0 \\
LATCH & 1 & 0 & 0 & 1 & 0 & 0 \\
\hline
\end{tabular}


Table 2-9 Matching results of scene 2 between aerial and MLS ortho-image of the 3rd and 4th iteration

\begin{tabular}{lccc|ccc} 
& & \multicolumn{3}{c|}{$3^{\text {rd }}$ run } & \multicolumn{3}{c}{$4^{\text {th }}$ run } \\
& Matches & Inliers & $\begin{array}{c}\text { Correct } \\
\text { Matches }\end{array}$ & Matches & Inliers & $\begin{array}{c}\text { Correct } \\
\text { Matches }\end{array}$ \\
\hline \hline SIFT & 51 & 9 & 0 & 51 & 9 & 1 \\
KAZE & 79 & 17 & 5 & 79 & 21 & 21 \\
AKAZE & 15 & 6 & 3 & 15 & 8 & 5 \\
LATCH & 3 & 0 & 0 & 3 & 0 & 0 \\
\hline
\end{tabular}

\subsubsection{Discussion}

\subsubsection{Conclusion}

This part of the chapter addressed the topic of tie feature extraction within the framework of the registration of aerial nadir images, mobile mapping panoramic images and MLS data. The aim of the overall project is to develop an automatic pipeline to correct the trajectory of mobile mapping platforms, especially in urban areas where reliable GNSS localisation is scarce. As a prerequisite for an orientation update of the platform's trajectory, precise tie information is needed. In this part, feature-based extraction techniques have been evaluated. It could be shown that the outcome highly depends on the algorithm itself and data pre-processing. KAZE seems to be the most reliable feature extraction method in both cases - mobile laser scanning intensity and panoramic imagery. SIFT and AKAZE only yield mediocre results, and do not benefit from resampling and blurring the images to the same extent as KAZE does. Although the Förstner-Operator detects good and significant features, LATCH failed to describe them accordingly to allow for a successful matching. In our scenario, binary descriptors are not as powerful coping with changes in illumination and contrast as float descriptors, and cannot manage to handle features originating from different sensors that well. However, due to this specific setup, and a very generic feature matching, further tests have to be conducted to draw a thorough conclusion on binary descriptors' performance. Interestingly, the discussed techniques show a better performance in conjunction with MLS and aerial data than with MMI data.

\subsubsection{Outlook}

Evidently, repetitive patterns of road markings are the biggest obstacle for a successful registration of the data sets. To tackle this issue efficiently, spatial information has to be introduced. Although the positioning accuracy of mobile platforms may be diminished in urban areas, their exterior orientation could support feature matching. In other words, they can be utilised to introduce search constraints as they allow for the localisation of individual keypoints. Besides that, using contextual information and shape knowledge can augment 
feature description to prevent mismatches. Additionally, nonessential descriptor invariances or capabilities could be removed from the respective original implementations (e.g. rotational \& scale invariance, sub-pixel localisation etc.). As far as MLS data is concerned, utilising high-level feature extraction methods could further facilitate the registration process also in areas with a lower point density and therefore fewer distinct keypoints. For this reason, entities, such as kerbstones or entire zebra crossings, can be utilised to accomplish this task.

\subsection{Part 2 - Advanced tie feature matching for the registration of mobile mapping imaging data and aerial imagery}

\subsubsection{Abstract}

Mobile mapping's ability to acquire high-resolution ground data is opposing unreliable localisation capabilities of satellite-based positioning systems in urban areas. Buildings shape canyons impeding a direct line-of-sight to navigation satellites resulting in a deficiency to accurately estimate the mobile platform's position. Consequently, acquired data products' positioning quality is considerably diminished. This issue has been widely addressed in the literature and research projects. However, a consistent compliance of subdecimetre accuracy as well as a correction of errors in height remain unsolved.

We propose a novel approach to enhance Mobile mapping (MM) image orientation based on the utilisation of highly accurate orientation parameters derived from aerial imagery. In addition to that, the diminished exterior orientation parameters of the MM platform will be utilised as they enable the application of accurate matching techniques needed to derive reliable tie information. This tie information will then be used within an adjustment solution to correct affected MM data.

This part of the chapter presents an advanced feature matching procedure as a prerequisite to the aforementioned orientation update. MM data is orthoprojected to gain a higher resemblance to aerial nadir data simplifying the images' geometry for matching. By utilising MM exterior orientation parameters, search windows may be used in conjunction with a selective keypoint detection and template matching. Originating from different sensor systems, however, difficulties arise with respect to changes in illumination, radiometry and a different original perspective. To respond to these challenges for feature detection, the procedure relies on detecting keypoints in only one image. 
Initial tests indicate a considerable improvement in comparison to classic detector/descriptor approaches in this particular matching scenario. This method leads to a significant reduction of outliers due to the limited availability of putative matches and the utilisation of templates instead of feature descriptors. In our experiments discussed in this part, typical urban scenes have been used for evaluating the proposed method. Even though no additional outlier removal techniques have been used, our method yields almost $90 \%$ of correct correspondences. However, repetitive image patterns may still induce ambiguities which cannot be fully averted by this technique. Hence and besides, possible advancements will be briefly presented.

\subsubsection{Introduction}

Mobile mapping data products are a valuable, additional source of geoinformation especially to extend coverage and enhance detail in urban areas. However, these areas in particular cause difficulties for satellite-based direct georeferencing techniques, if buildings or other tall structures obstruct the necessary line-of-sight between the MM platform and the respective navigation satellites. Hence, MM data products' absolute accuracy may be impaired.

To tackle that problem, many authors rely on other sources of exterior orientation information, such as digital maps, aerial imagery or ground control points (Ji et al. (2015); Jaud et al. (2013); Kümmerle et al. (2011); Levinson and Thrun (2007)). A similarity of available approaches is a registration between the MM data and the reference data to yield enough correspondences which are utilised as constraints in an adjustment or filter solution, respectively. The majority of these methods utilises mobile laser scanning data, and approaches relying on MM images, such as Ji et al. (2015) compensate for matching errors within the filtering stage rendering a reliable registration unnecessary by accepting correct but mediocre correspondences. Moreover, these methods do not compensate for vertical errors, and cannot comply with a consistent decimetre accuracy.

In our research project, high-resolution aerial nadir and oblique images will be used to provide the required exterior orientation parameters. Although this part of the chapter will focus solely on the registration between MM and orthoimages computed from aerial nadir images, it constitutes the basis for further developments with respect to the registration of $\mathrm{MM}$ and oblique images. A registration of images with a non-standard geometry and different sensor setup is not a trivial task. Either these differences have to be accounted for by feature detectors and descriptors, or by pre-processing to converge the images. State-of-the-art algorithms for the extraction of feature keypoints can account for differences in scale, rotation, illumination and perspective all to a certain degree (Alcanterilla et al. (2013); Rublee et al. (2011); Levi and 
Hassner (2015)), but bridging great overall variations e.g. between MM and aerial images has not been achieved yet.

Thus, this part will discuss a matching strategy based on a pre-processing step to approximate the MM data to the aerial nadir image by performing an orthoprojection of the panoramic MM image. Moreover, the MM image's resolution and sharpness is also adjusted to increase the resemblance to the aerial reference data. Although the exterior orientation of the MM data may be imprecise, it is used to confine the matching procedure. However, the similarity between aerial nadir and MM images is still limited due to variations of contrast, illumination, image content, original perspective etc., reducing the efficiency of feature-based matching considerably. Road markings, however, remain a common element for image registration in urban areas with the intrinsic disadvantage to be repetitive. Consequently, a feature-based matching may fail to find the correct correspondences due to the ambiguity of the computed feature description.

Our experiments in the past have shown (Jende et al., 2016a), that repetitive road markings can prevent a correct registration regardless of the employed detector/descriptor combination or the exclusion of various invariances. In conclusion, a feature matching relying on a separate detection and description phase in both input images is immanently sensitive to the overall image differences in this non-standard scenario. Therefore, the strategy presented in this part of the chapter comprises a feature detection only in the aerial image, a kd-tree neighbourhood search for areas with sparse keypoints and a crosscorrelation based template matching. Using an area-based approach is highly facilitated by the images' similar perspective as the integration of invariances, such as change in perspective, large scale differences or rotation is nonessential. In addition to that, the procedure can be designed independently from a feature detection in two images which enables the definition of the size of the moving window. Moreover, a template may alleviate differences in image details to a better extent than descriptor-based approaches, and consequently allows for a bridging of sensor differences. Even though this method yields only a few, but reliable correspondences, it allows for the determination of initial transformation parameters. Depending on the distribution and the number of correspondences, they may serve either as an outlier mask for a subsequent feature matching or already as an input for an adjustment to rectify the MM data set. 


\subsubsection{Method}

\subsubsection{Pre-processing \\ Pre-processing of aerial images}

Our test data set comprises 15 aerial nadir images of Rotterdam (NL) with a maximum overlap of 6 images. These images were all combined to an orthomosaic with a ground sampling distance of 12 centimetres.

\section{Ortho-projection and blurring of MM image}

As a necessary pre-processing step, the MM data is ortho-projected in order to decrease the perspective differences between the input images. A horizontal plane representing the actual ground is computed based on the location of the MM sensor and its fixed height above the ground. A grid spacing that equals the aerial ortho-image's resolution is defined, and the corresponding MM panoramic image's pixels are projected onto the ground plane. Due to the definite description of the plane, all pixels contain approximated world coordinates. However, if the surrounding surface around the MM recording location is non-planar, this projection may lead to distortions. Although the deviation of the computed plane from the actual surface is minor especially in the immediate vicinity of the MM platform, a strategy has been developed to account for this issue. Belonging to the task of adjustment, however, this method will be presented in the future. After the ortho-projection, the MM image is blurred using a Gaussian filter to increase the resemblance to the aerial image for the step of template matching.

\subsubsection{Matching and estimation of transformation}

\section{Feature detection}

Feature keypoints are only detected in the aerial ortho-image using a modified version of the Förstner operator (Förstner and Gülch, 1987) proposed by Köthe (2003a). The major advancement is a revision of the structure tensor to extract corners at twice the resolution of the original image. This results in a higher accuracy and quality of the identified corners.

Corner features are suitable to represent road markings and other geometrical structures on the ground. To circumvent individual parametrisations as well as the risk of overfitting feature detection for a specific data set and first of foremost as a consistent and reliable keypoint detection in two disparate images cannot be guaranteed, feature keypoints are only detected in the aerial image. Back-projecting the identified keypoints into the MM image in a later 
step and using a template for matching, renders feature detection in the second image dispensable.

\section{Avoiding repetitive patterns}

Subsequently to feature detection, a kd-tree is computed to organise the detected keypoints and to allow for neighbourhood search. Repetitive road markings (see e.g. Figure 2.17) consist of many corners, thus returning many keypoints. Therefore, a kd-tree is used to identify isolated keypoints with fewer neighbours (i.e. 7 keypoints) in its extended surrounding of 50 by 50 pixels or 6 by 6 metres. This strategy simply avoids employing keypoints detected at corners of repetitive road markings for matching. In the future, however, these difficult features should be also utilised for a registration. A possible approach will be discussed in the last section of this part of the chapter.

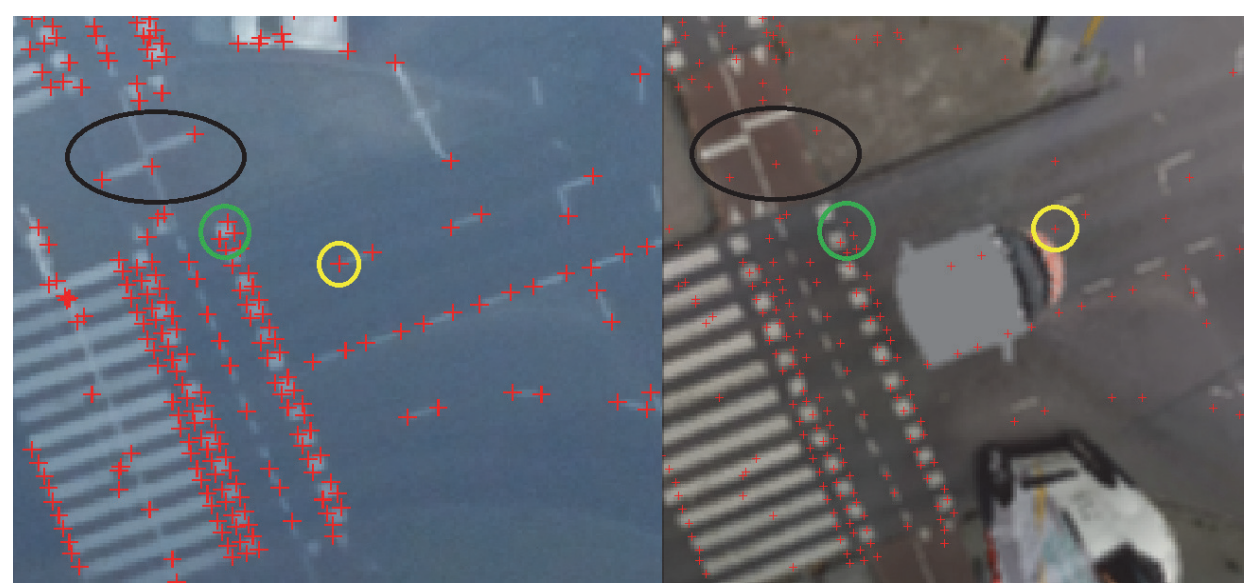

Figure 2.17 Left: aerial image with extracted Förstner keypoints; Right: aerial image's keypoints back-projected into MM image. Coloured circles illustrate the horizontal error. Moreover, the ambiguity problem with repetitive road markings becomes apparent using the example of the green circle; three corners have been identified in the aerial image. The back-projected keypoints, however, are now closer to the adjacent square-shaped road marking which may lead to a wrong correspondence.

\section{Template Matching}

By back-projecting the aerial image's keypoints into the MM image, the horizontal offset between the data sets becomes visible (Figure 2.17). Subsequently, for every keypoint in the aerial image, a template with 16 by 16 pixels is defined. To constrain the search space by still taking enough error margin into account, a 40 by 40 pixel neighbourhood around the backprojected keypoint in the MM ortho-image is used. Future work will, however, allow for an automatic and flexible definition of this window to account for different magnitudes of error in the MM image. Moreover, another feasible solution for the future would involve using parts of the MM image as a template, 
thus designing the process vice versa. Corresponding parts of the image are now cross-correlated. A threshold determines whether the peak of the normalised values in the cross-correlation matrix indicates a valid match. Moreover, the location of the peak is assumed to correspond to the extracted keypoint in the aerial image, thus the back-projected keypoint is moved to the peak's position allowing for a pixel-to-pixel accurate correspondence between the two images.

\section{Estimation of transformation}

The aerial and the MM image share approximately the same projection reducing the degrees of freedom to translation, uniform scaling and rotation. Scale is induced by an estimated ground sampling distance of the aerial orthoimage and rotation by a little deviation of the yaw axis of the MM platform from the north orientation. Thus, translation is the most crucial parameter.

As a similarity transform with only four degrees of freedom, two point pairs are needed to compute the parameters. The resulting transformation can be then employed as an outlier mask for a subsequent feature-based matching approach using e.g. state-of-the-art detector/descriptor combinations, such as those mentioned in the introduction. Since this approach finds an optimal solution with a least squares estimation, more than two non-collinear point correspondences can be used with the disadvantage of being not very robust against outliers. However, further experiments will show if the computed transform is reliable enough to directly serve as an input for the orientation update to rectify the MM data.

\subsubsection{Experimental Study}

\subsubsection{Experimental Setup}

For this research project, the entire city of Rotterdam (NL) is being used as a test area as it offers a typical urban canyon scenario with a great number of high-rise buildings. For this specific test case, however, 14 tiles in Rotterdam's city centre cropped from the aerial ortho-image have been selected (Figure 2.18). These 14 tiles include difficult lighting and contrast conditions, repetitive road markings as well as great differences in the image content due to bustling traffic and vegetation. For every aerial image tile its centre coordinate is used to retrieve the corresponding MM ortho-image with an extent of approximately 21 metres side length. The presented approach has been applied to every tile of the test case, and no additional outlier removal technique has been conducted nor has the parametrisation been changed. In order to evaluate the performance of the proposed method, visual checks as well as a comparison with feature-based approaches were conducted. For that purpose, a combination of the AGAST corner detector (Mair et al., 2010a) and SURF will 
be used (Bay et al., 2008). AGAST is a corner detector which is not scale nor rotational invariant. This is intended as the rotation and scale of keypoints at a local level in this setup is negligible, and would introduce unnecessary ambiguities to the feature description. SURF is a widely-used float descriptor, which is - as default - scale and rotational invariant, but both parameters are estimated in the detector phase. Thus, by having no such information available from AGAST, SURF cannot account for these invariances. Alternatively, rotational invariance could be achieved by using Upright-SIFT (ibid). Moreover, coarse orientation information is also provided for the matching step by defining a search window around a back-projected aerial image keypoint, reducing the number of possible matches accordingly.

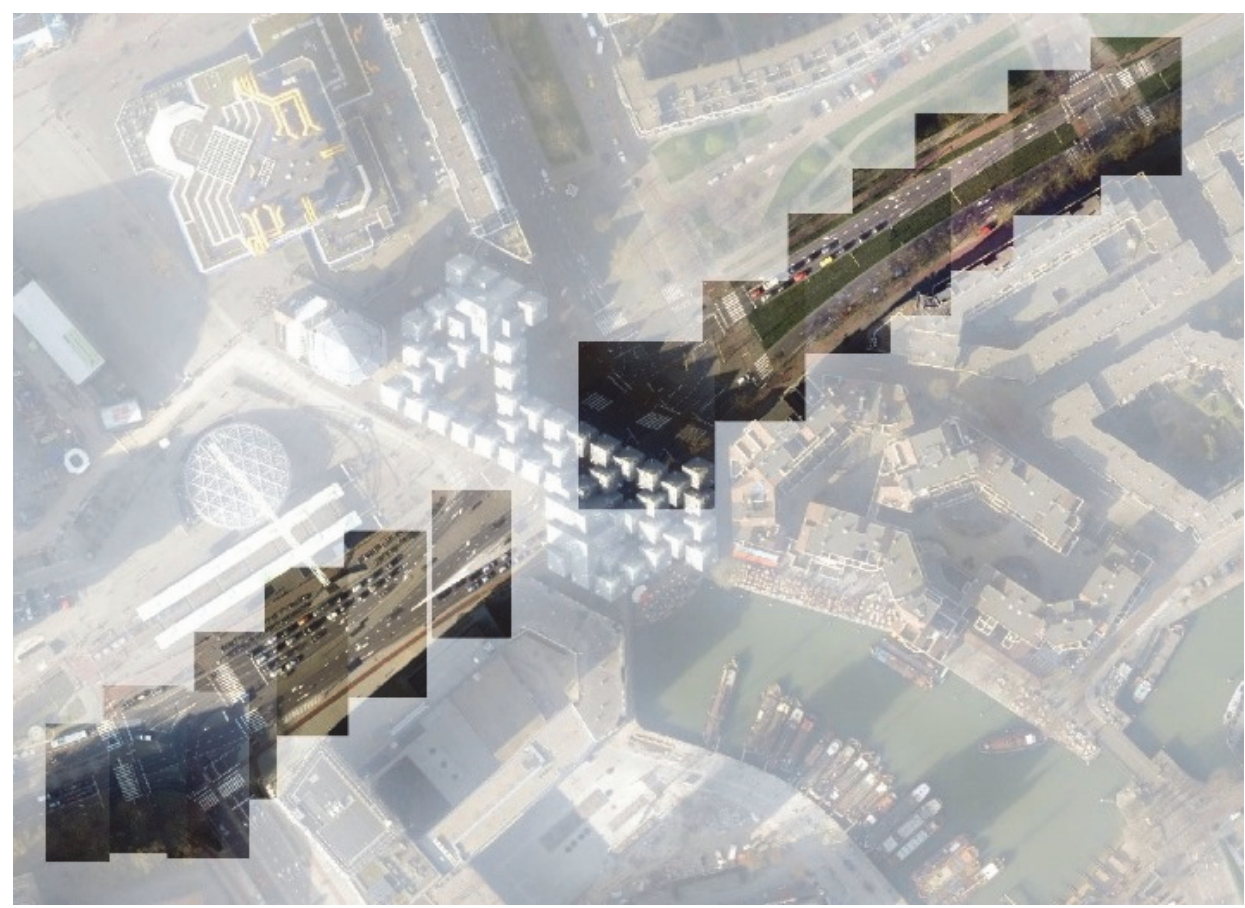

Figure 2.18 Test site in Rotterdam (the gap is caused by a building spanning the road).

Additionally, a second feature-based matching approach with Förstner keypoint detection (Köthe, 2003a) and SURF description will be used for the comparison. For both methods, their default detector and descriptor parameter sets and a RANSAC-based outlier removal have been used. The inliers are then compared against the matching result of the proposed method. It is evident, that the remaining matches might be wrong, if RANSAC converged to a wrong solution. Involving all matches, however, would make a comparison difficult and insignificant as these two methods return far more matches than the proposed method. 
To interpret the results correctly, it has to be mentioned, that the aim of the proposed method is to be as reliable as possible to find an initial estimate of the transformation between the aerial nadir and MM ortho-image. Thus, if a good ratio of matches versus inliers is available, the estimated transformation is more likely to be correct. Consequently, a few good matches are favoured over a high number of mediocre matches. To this end, the visual check has been quite strict, only labelling correct corner-to-corner or rather point-topoint correspondences as valid. However, a registration between MM and aerial data might not always be possible due to the lack of common features or different image content etc. Since the entire workflow includes a registration among adjacent MM images, this issue is not very crucial as a MM tile missing a direct correspondence to the aerial reference can be bridged accordingly.

\subsubsection{Overview of experimental results}

The three charts below (Figure 2.19, Figure 2.20 \& Figure 2.21) show the overall results of the conducted experiment. In general, the number of matches varies among the three methods. But more importantly, the number of correct correspondences diverges significantly (see Table 2-10).

Table 2-10 Summary of matches, inliers and averages of all test tiles.

\begin{tabular}{lcccc|c}
\hline & $\begin{array}{c}\text { Number of } \\
\text { matches }\end{array}$ & $\begin{array}{c}\text { Number } \\
\text { of inliers }\end{array}$ & $\begin{array}{c}\text { Average } \\
\text { matches/tile }\end{array}$ & $\begin{array}{c}\text { Average } \\
\text { inliers/tile }\end{array}$ & $\begin{array}{c}\text { Ratio } \\
\text { (in \%) }\end{array}$ \\
\hline \hline Förstner/CC & 86 & 75 & 6,1 & 5,4 & 87.2 \\
AGAST /SURF & 243 & 58 & 17,4 & 4,1 & 23.9 \\
Förstner/SURF & 172 & 9 & 12,3 & 0,6 & 5.2 \\
\hline
\end{tabular}

With almost $90 \%$ of correct correspondences of the total number of identified matches, the presented method clearly outperforms the other methods which rely on a classic feature detector/descriptor combination. Matching images with a non-standard geometry is indeed quite difficult, even though coarse orientation parameters could be utilised. The aim, however, is to develop a method to be very reliable with respect to deriving an initial transformation between the aerial nadir and MM ortho-image.

In case of the presented method, a transformation estimate could be derived in 9 out of 14 tiles, resulting in a success rate of $64 \%$. There were, admittedly, two tiles ( 5 \& 13) without any correspondences making an estimation impossible. 


\section{Förstner/cross-correlation}

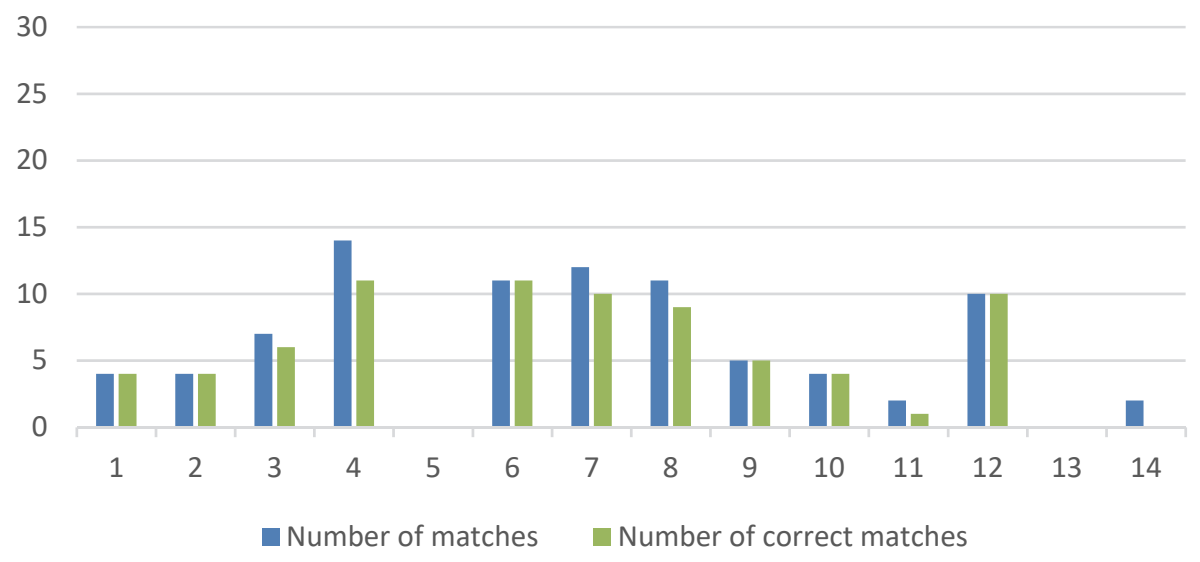

Figure 2.19 Matching results of proposed method across all 14 tiles.

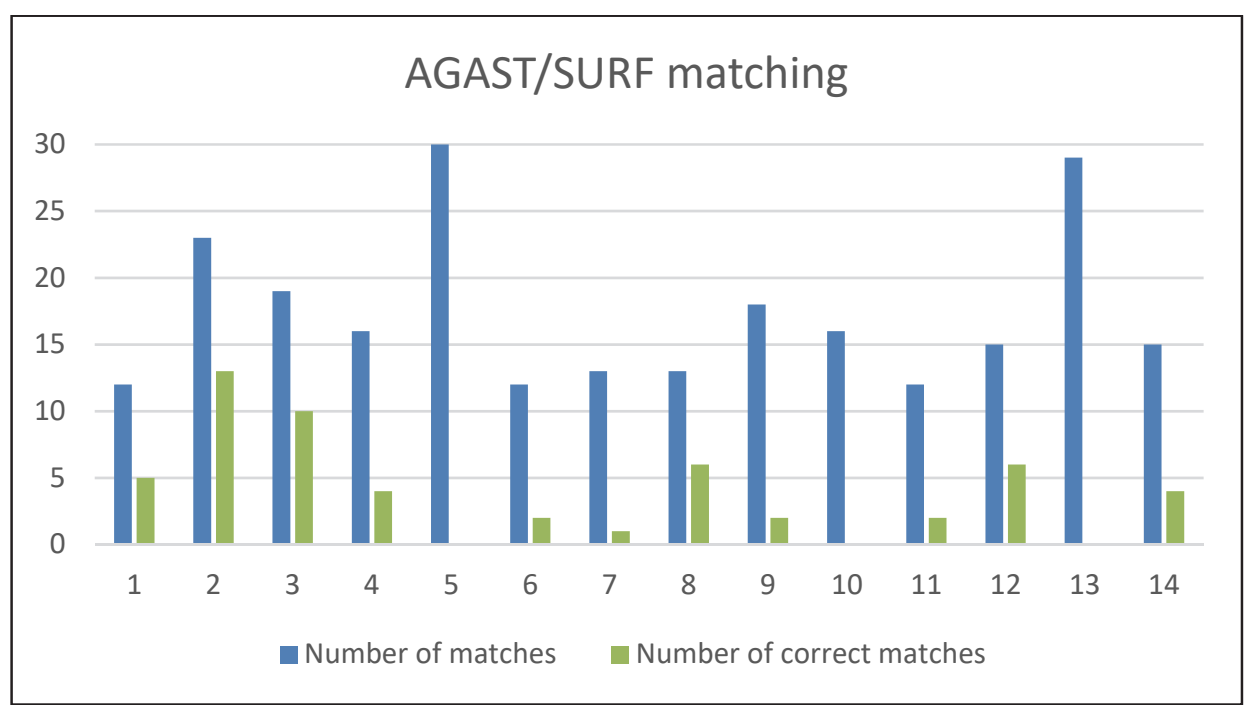

Figure 2.20 Matching results of AGAST detection and SURF description across all 14 tiles. 


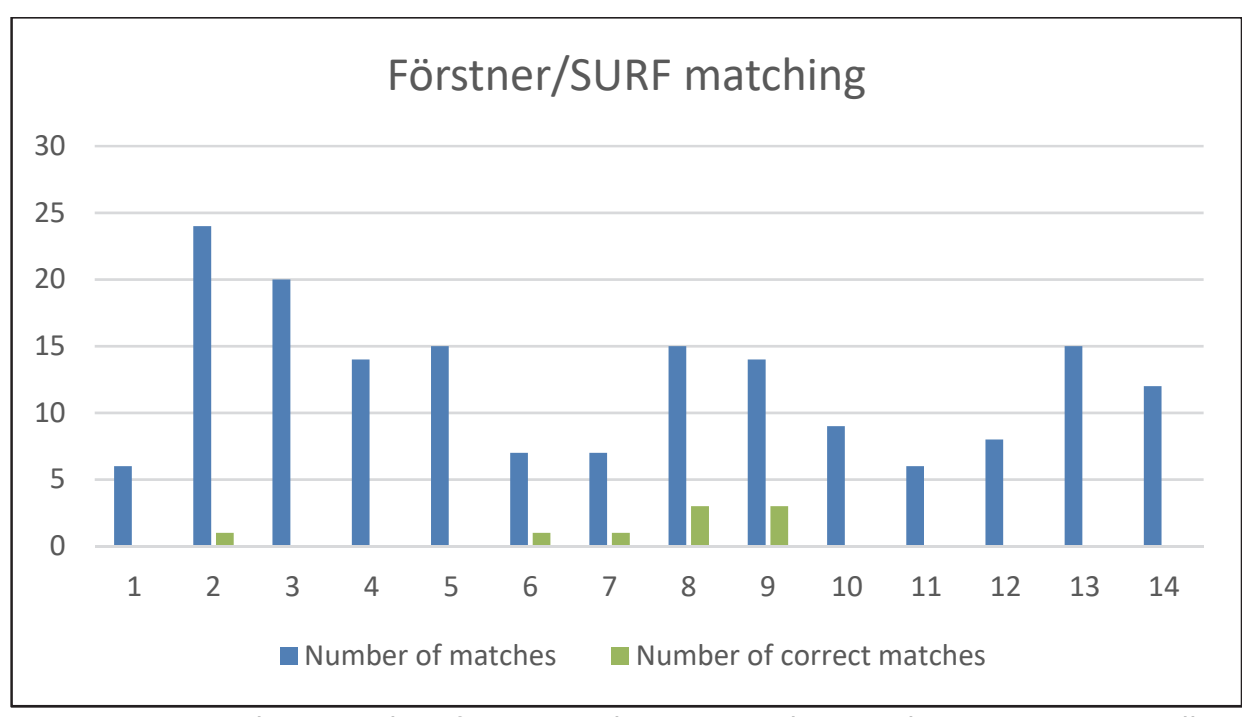

Figure 2.21 Matching results of Förstner detection and SURF description across all 14 tiles.

\subsubsection{Discussion of experimental results}

In this section, some examples will be shown and discussed with regard to the individual performance of the respective method. In the figures shown below, red indicates a wrong and green a correct match.

\section{Tile 2}

Tile 2 shows a typical urban scenario with a zebra crossing. These repetitive road markings hamper the computation of distinct keypoint descriptors considerably. Figure 2.22 shows the matching result achieved with an AGAST detection and a SURF description. The result is good with only 10 mismatches out of 23 correspondences found in total. On the other hand, the result obtained by the method presented in this part shown in Figure 2.23 only found 4 , but therefore only correct correspondences. Figure 2.24 depicts the results achieved with a Förstner corner detection and a SURF description. It is clear, that the repetitive appearance of the road markings hinders a successful registration. Among 24 matches, there is not a single correct correspondence. 


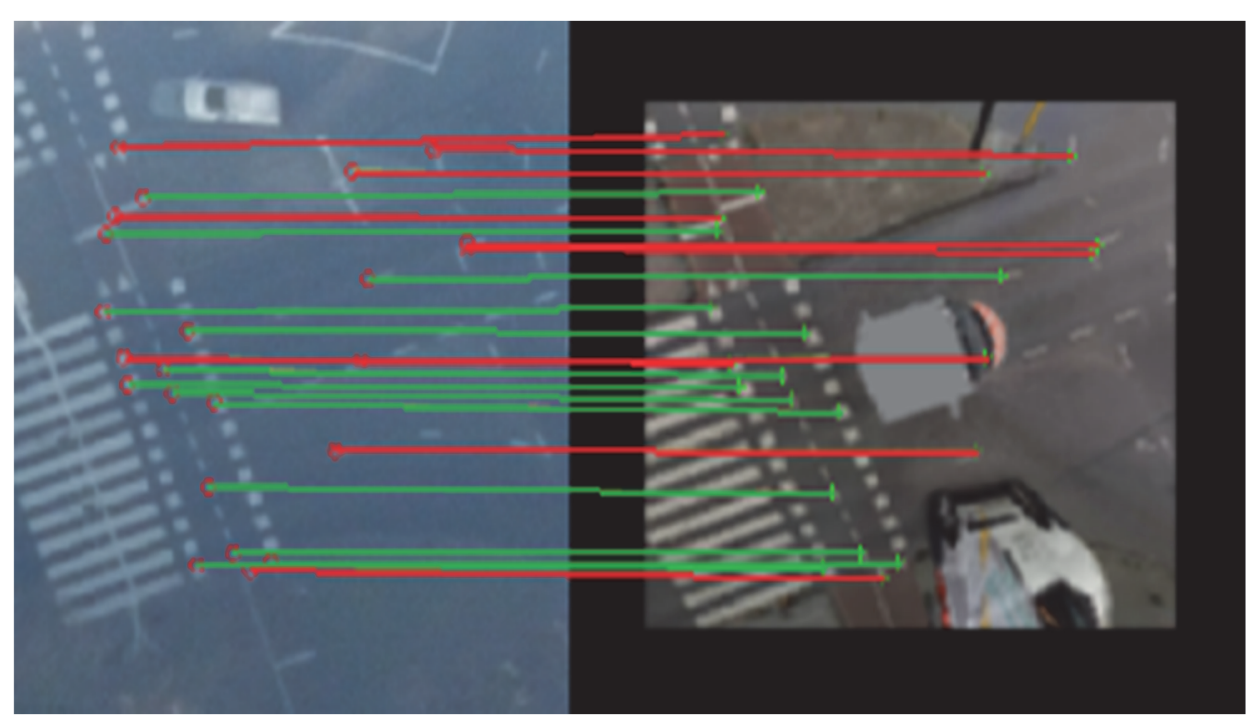

Figure 2.22 AGAST/SURF matching result of 2 nd tile.

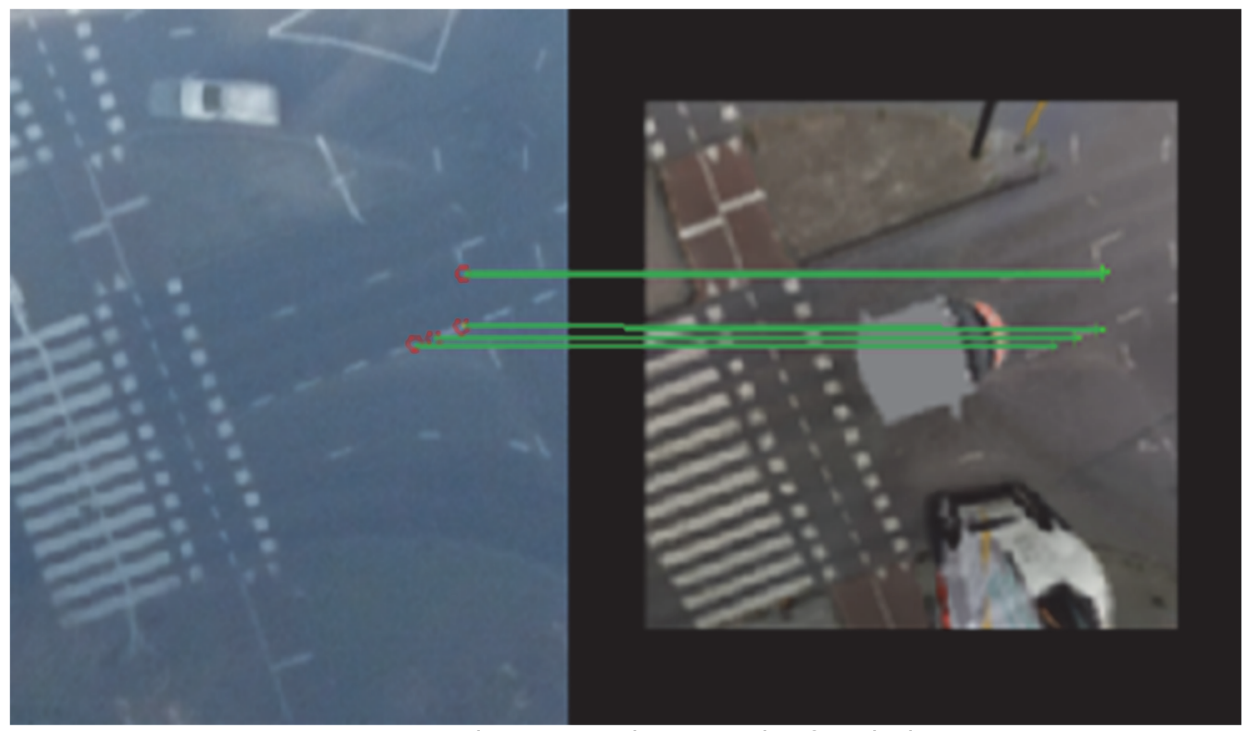

Figure 2.23 Förstner Cross-Correlation matching result of 2nd tile. 


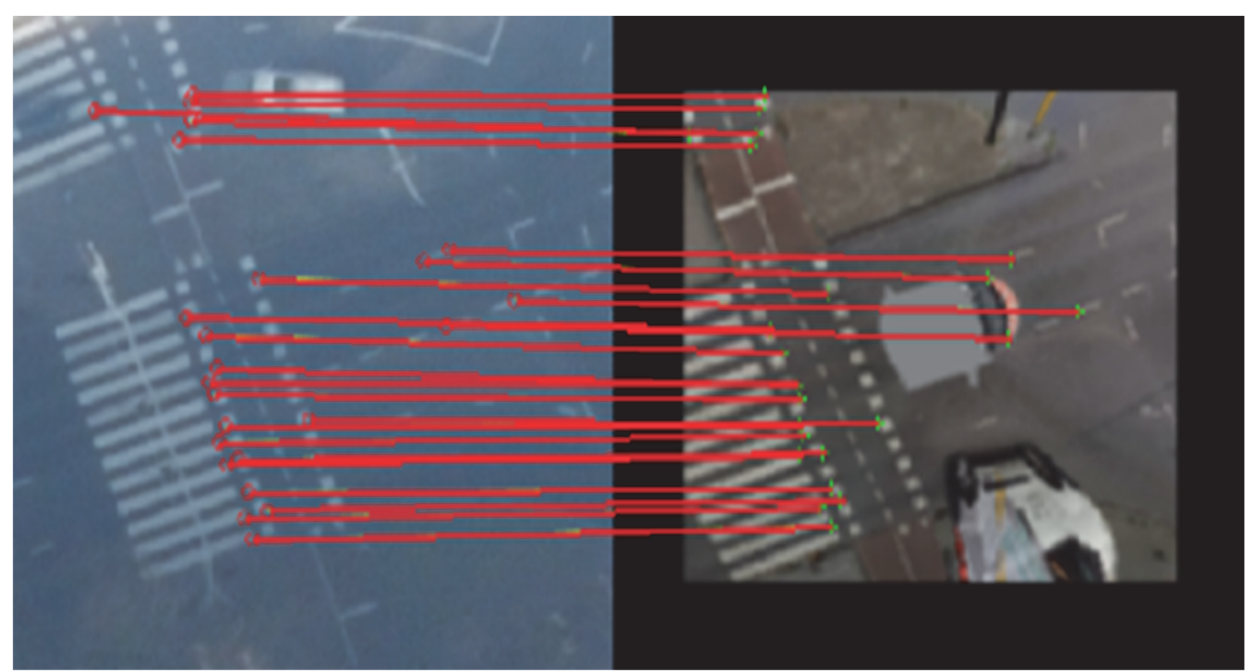

Figure 2.24 Förstner/SURF matching result of 2nd tile.

\section{Tile 6}

The result shown in Figure 2.25 is quite interesting as it ideally illustrates the problem with feature detection in two images from different sensors. Due to the overall differences, but the same parametrisation of the keypoint detection method, different keypoints are returned (see Figure 2.26) resulting in wrong correspondences. In this case, only 2 out of 12 matches were correct. Resolving this issue by adapting the feature detector's parameters to each image data set, however, may involve the risk of overfitting.

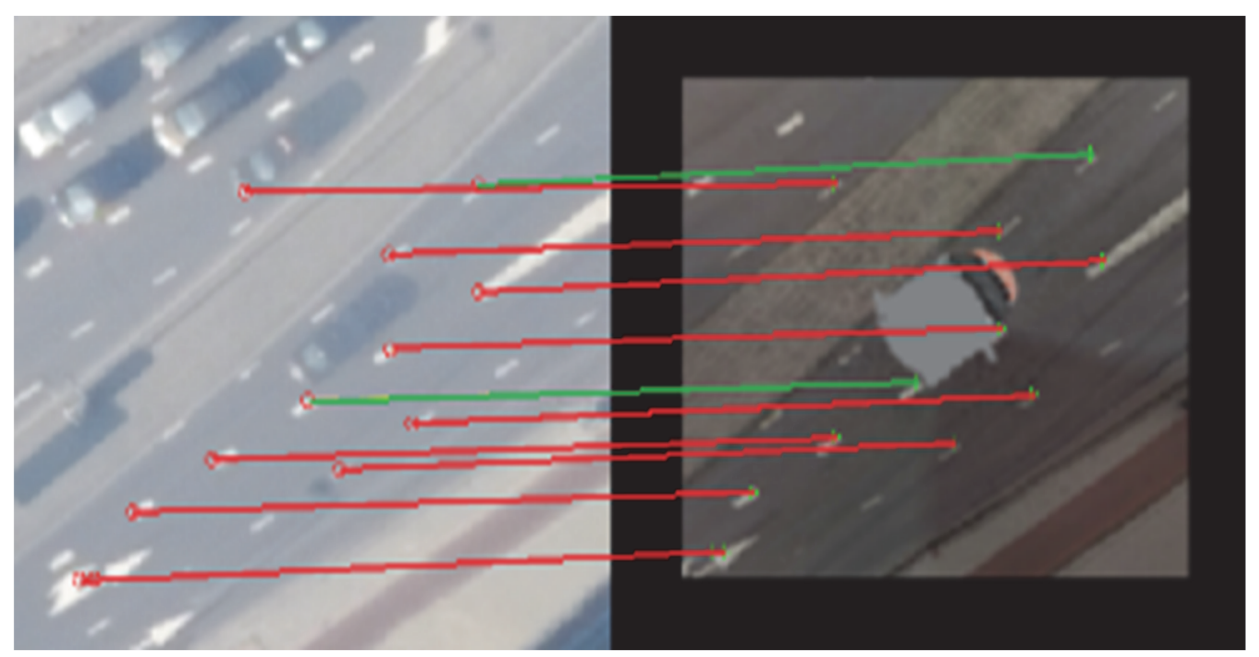

Figure 2.25 AGAST/SURF matching result of 6 th tile. 

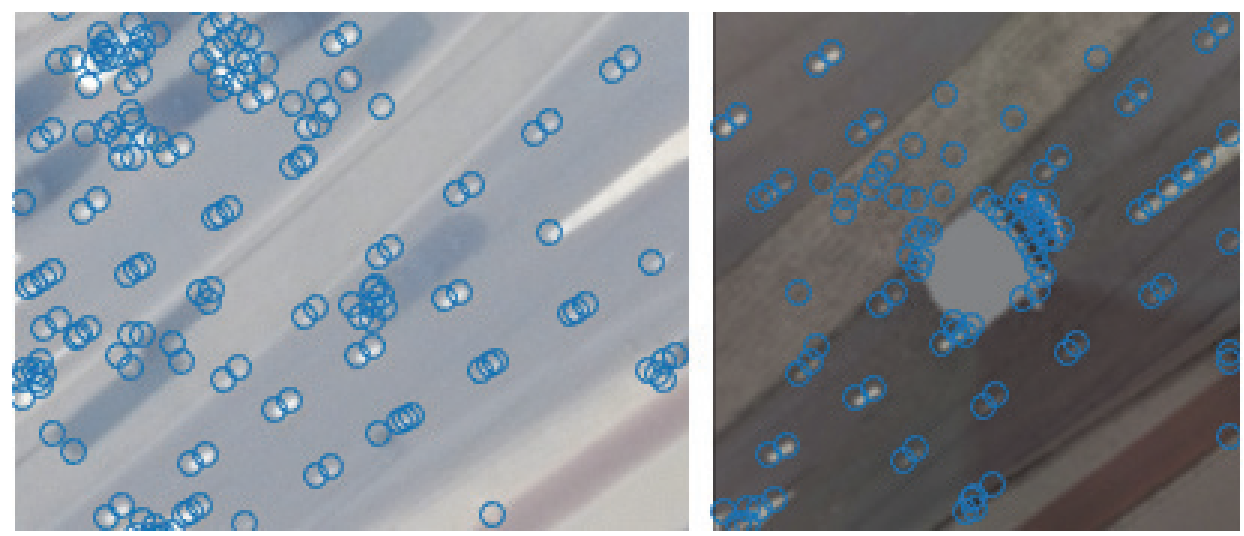

Figure 2.26 AGAST keypoints in aerial nadir and MM ortho-image.

The presented method can compensate for these kind of problems by having a keypoint detection in only one image leading to a substantially better result of 11 correct correspondences (Figure 2.27).

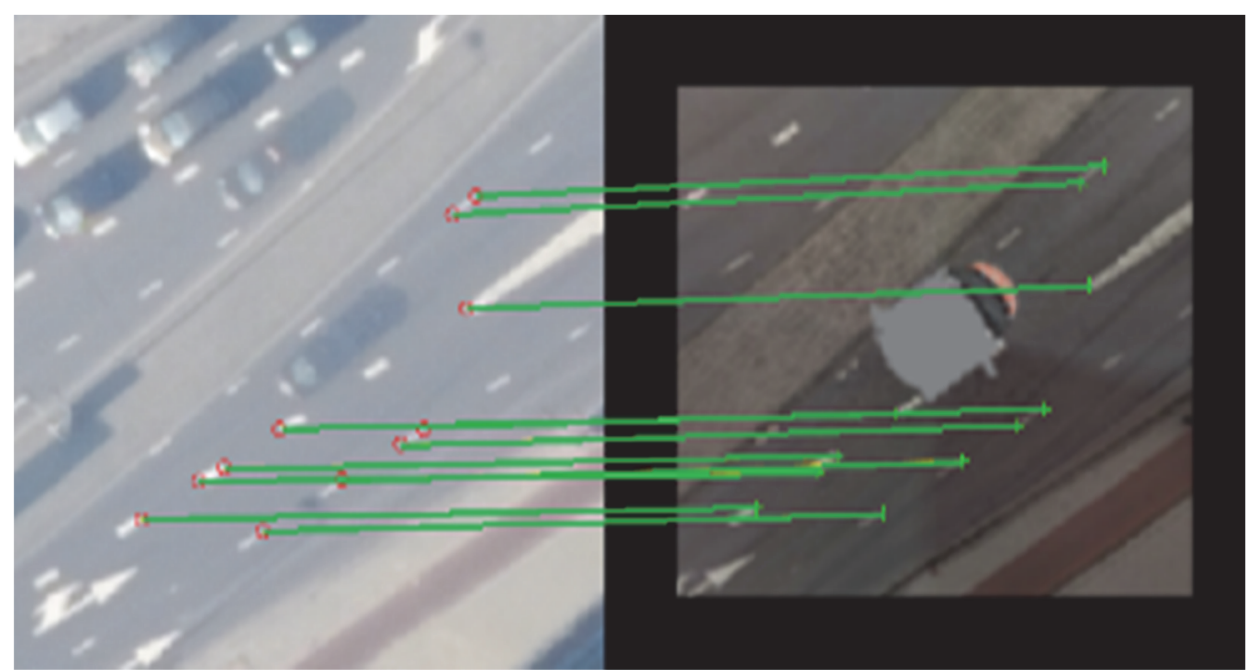

Figure 2.27 Förstner Cross-Correlation matching result of 6th tile.

\section{Tile 8}

Not only different sensor systems lead to difficulties in matching, but also changing image content. A match is either valid, if the correlation value is above a certain threshold or below a defined descriptor distance. Since all three methods utilise some sort of search window, the number of matching candidates is already reduced to a high degree. If however, the image content changes - in that case - the MM car covering the zebra crossing, the closest and highest correlation value are the neighbouring strips of the zebra crossing (Figure 2.28). This can be tackled by either adjusting the correlation threshold 
or introducing a constraint preventing the assignment of a match which is below a certain clearance.

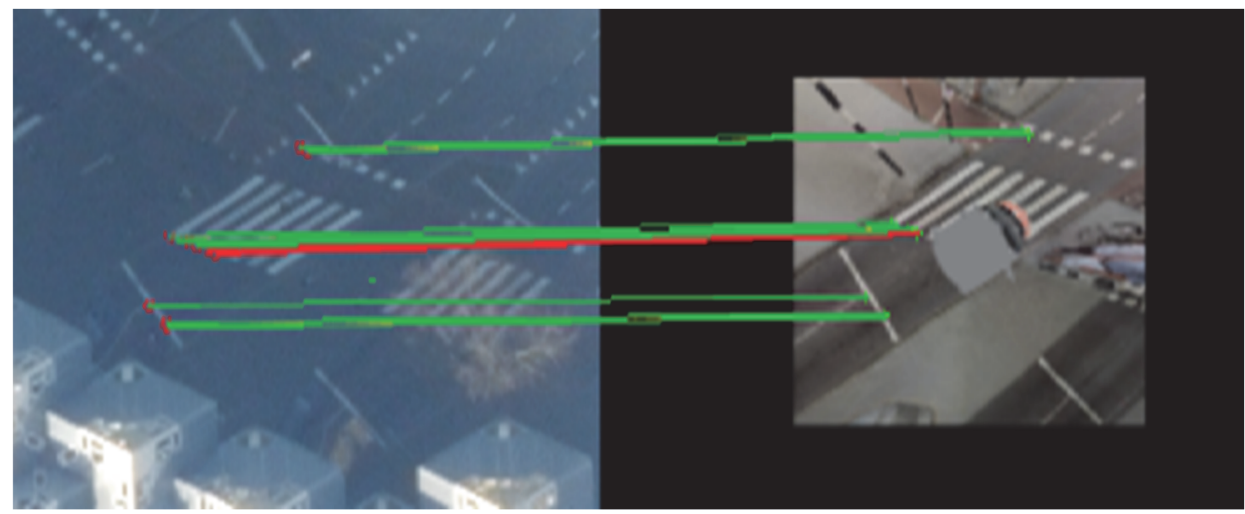

Figure 2.28 Förstner Cross-Correlation matching result of 8th tile.

\section{Tile 9}

Tile 9 comprises of many elements impeding a successful matching. Vegetation overgrows certain parts in the aerial nadir image, repetitive road markings and a great difference in contrast. Figure 2.29 shows the result of the Förstner detection in combination with the SURF description. Out of 14 matches in total, just 3 are valid correspondences.

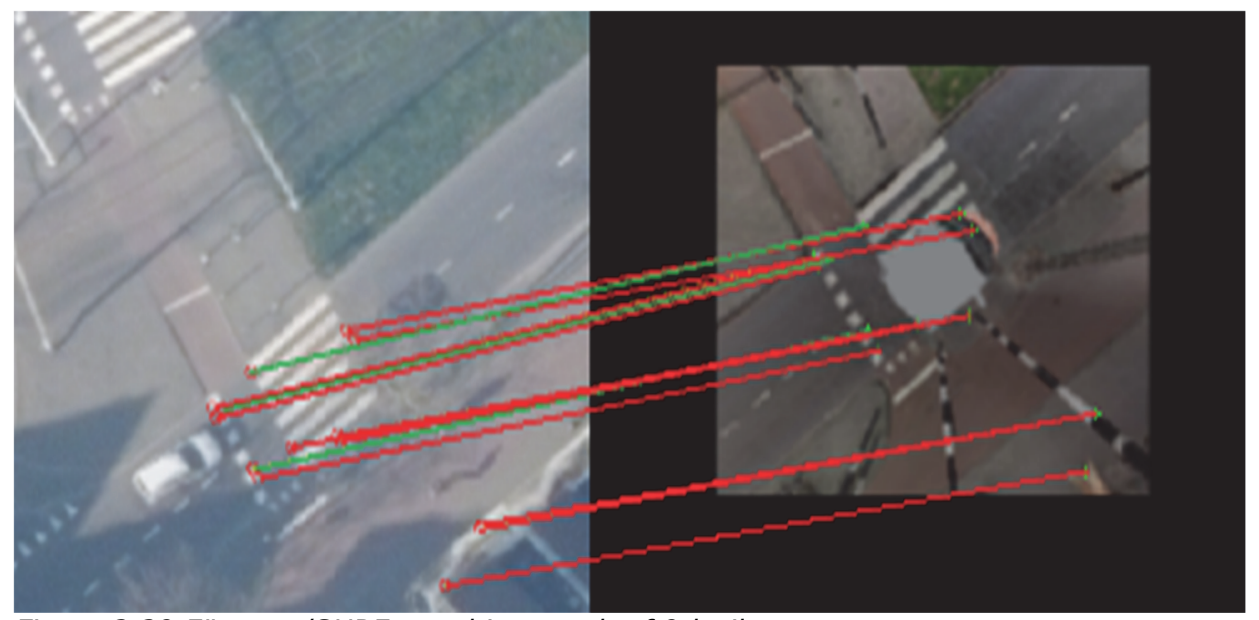

Figure 2.29 Förstner/SURF matching result of 9th tile.

With the introduction of a keypoint neighbourhood constraint preventing a template matching in an area with a high keypoint density, the proposed method is able to identify only correct correspondences between these two images (Figure 2.30). 


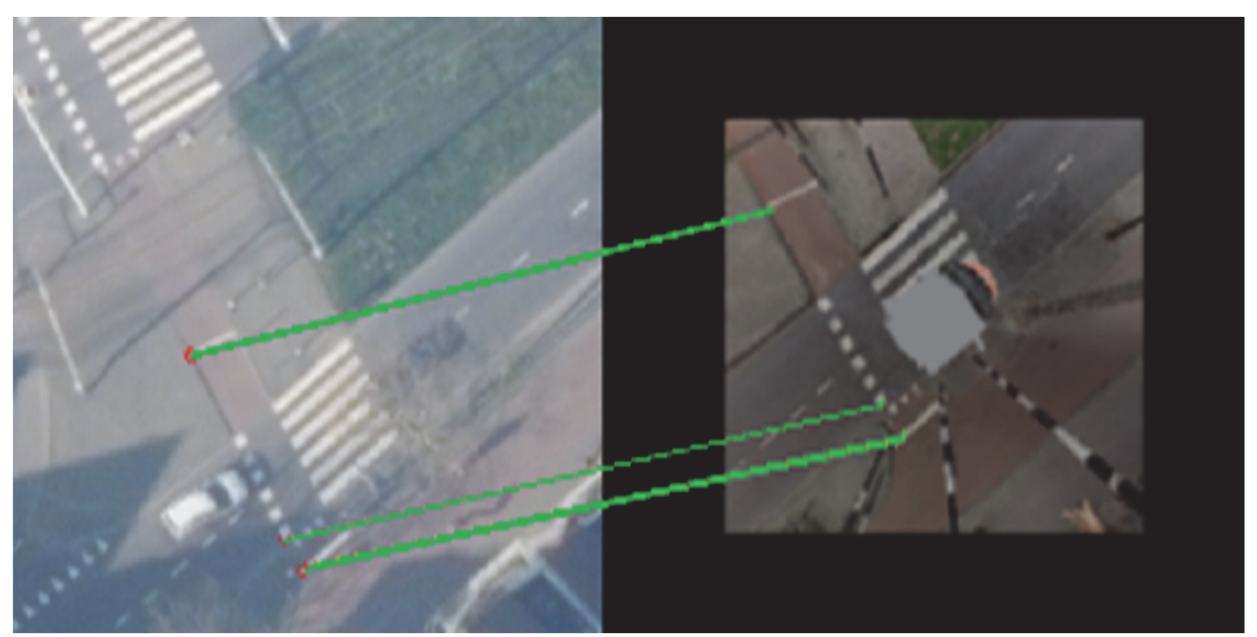

Figure 2.30 Förstner Cross-Correlation matching result of 9th tile.

\section{Tile 12}

This example is quite similar to tile 6 where a successful registration has to rely mainly on sparse road markings. Classic detector/descriptor approaches do most likely fail in such a scenario since computed descriptors are not unique, and keypoints detected across two images originating from different sensors may not be the identical.

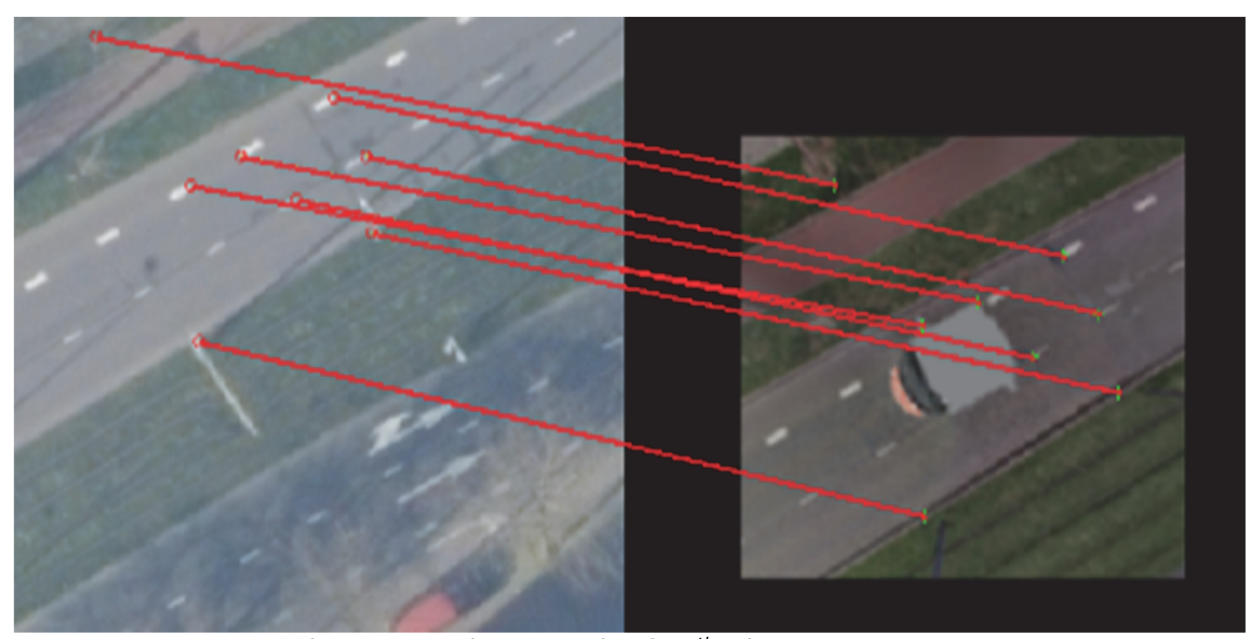

Figure 2.31 Förstner/SURF matching result of $12^{\text {th }}$ tile.

Comparing Figure 2.31 and Figure 2.32, it becomes evident that a crosscorrelation based matching in combination with a feature detection in only one image is more reliable and robust than a descriptor-based approach. 


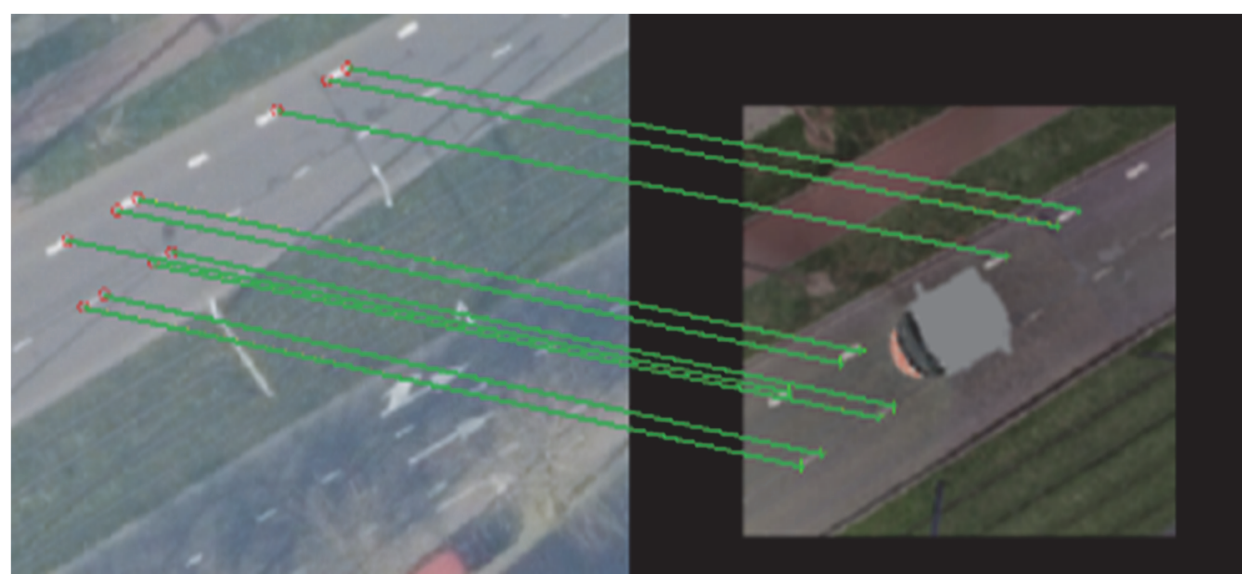

Figure 2.32 Förstner Cross-Correlation matching result of $12^{\text {th }}$ tile.

\section{Tile 13}

The method proposed in this part of the chapter did not return any matches with the data from tile 13, but the other two methods did not succeed either, even though a lot of matches but no correct correspondences have been identified. Again repetitive road markings, overgrowing vegetation and different image content prevent a successful registration (Figure 2.33).

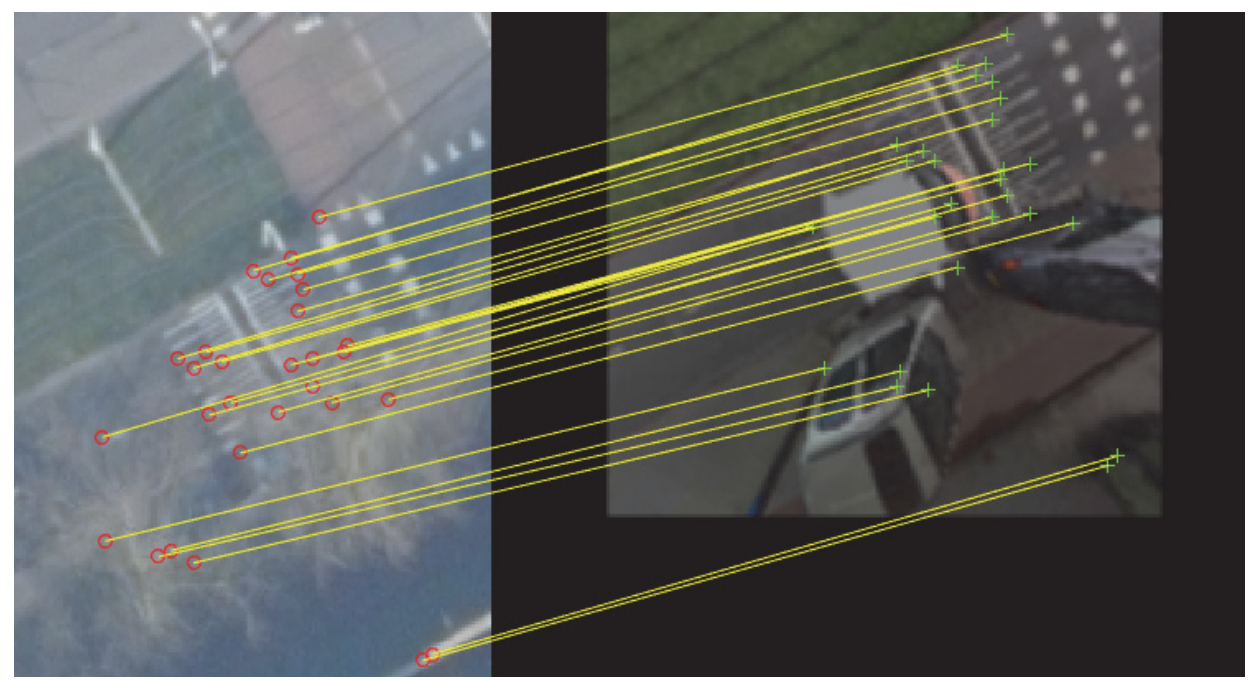

Figure 2.33 AGAST/SURF matching result of 13th tile with not a single correct correspondence.

\subsubsection{Conclusion}

A substantial part within this research project is a reliable registration between MM images and the reference data set. In order to solve a matching problem 
involving this non-standard geometry, standard approaches are likely to fail or do not yield reliable nor consistent results. Converging the images by orthoprojecting the panoramic MM image was a first important step to simplify the images' geometry for matching. The second important strategy was to exploit the images' orientation parameters which enable the use of search windows to reduce the number of matching candidates. In this part of the chapter, the emphasis has been laid on the third step to detect features in only one image, on the one hand, and to use a template matching approach instead of a feature description on the other hand. The presented results and their comparison with descriptor-based methods could show that not just the quality of individual correspondences, but also the reliability and robustness could be increased. Moreover, in 9 out of 12 cases where matches have been identified, a transformation could be estimated.

Currently, a kd-tree is being used to identify areas with a low keypoint density. Certainly, this a fast solution to count keypoints in a window but will actually serve as the basis for further developments. A problem that has not been tackled yet is how to cope efficiently with repetitive road markings. In most of the cases, they can be simply avoided by this very neighbourhood search, but future developments should also exploit their geometric properties for registering the images. In the past, there were certain endeavours to determine which parts of an image belong together, such as perceptual grouping (Lowe, 1985). Even though the method should be kept as simple as possible, and high-level feature approaches are likely to be immoderate also regarding their computational costs (Tournaire et al., 2006), the geometric interrelation between neighbouring keypoints will be utilised to determine whether certain road markings are repetitive. To this end, a check may be introduced, if a tuple of keypoints is collinear and complies with a set of rules.

Additionally, a network of correspondences between aerial to MM image matches and matches among MM images will be designed. Firstly, correspondences between MM images could contribute to the adjustment as they allow for the correction of relative errors along the MM platform's trajectory, and secondly, they may recover the orientation of individual MM tiles which do not have a direct correspondence to the aerial image. 


\section{Chapter 3 - A fully automatic approach to register mobile mapping and airborne imagery to support the correction of platform trajectories in GNSS-denied urban areas $^{17}$}

\footnotetext{
${ }^{17}$ This chapter is based on:

Jende, P., Nex, F., Gerke, M., \& Vosselman, G. (2016) 'A fully automatic approach to register mobile mapping and airborne imagery to support the correction of platform trajectories in GNSS-denied urban areas', ISPRS Journal of Photogrammetry and Remote Sensing, Vol. 141, pp. 86-99, https://doi.org/10.1016/j.isprsjprs.2018.04.017
} 


\subsection{Abstract}

Mobile mapping solutions have become a significant extension to traditional data acquisition methods over the last years. Independently from the sensor carried by a platform, may it be laser scanners or cameras, high-resolution data postings are opposing a poor absolute localisation accuracy in urban areas due to GNSS occlusions and multipath effects. Potentially inaccurate position estimations are propagated by IMUs which are furthermore prone to drift effects.

Thus, reliable and accurate absolute positioning on a par with MM's high-quality data remains an open issue. Multiple and diverse approaches have shown promising potential to mitigate GNSS errors in urban areas, but cannot achieve decimetre accuracy, require manual effort, or have limitations with respect to costs and availability.

This chapter presents a fully automatic approach to support the correction of MM imaging data based on correspondences with airborne nadir images. These correspondences can be employed to correct the MM platform's orientation by an adjustment solution. Unlike $M M$ as such, aerial images do not suffer from GNSS occlusions, and their accuracy is usually verified by employing wellestablished methods using ground control points.

However, a registration between $M M$ and aerial images is a non-standard matching scenario, and requires several strategies to yield reliable and accurate correspondences. Scale, perspective and content strongly vary between both image sources, thus traditional feature matching methods may fail. To this end, the registration process is designed to focus on common and clearly distinguishable elements, such as road markings, manholes, or kerbstones. With a registration accuracy of about $98 \%$, reliable tie information between MM and aerial data can be derived. Even though, the adjustment strategy is not covered in its entirety in this chapter, accuracy results after adjustment will be presented. It will be shown that a decimetre accuracy is well achievable in a real data test scenario. 


\subsection{Introduction}

Mobile mapping is an intriguing technology, and augments other image sources in the field of photogrammetry, such as aerial photography, UAV imagery, or satellite images. It complements the photogrammetric portfolio of different resolutions, availability, and applications while facilitating the completeness of respective data products. Especially in urban areas, this key advantage comes into effect by enabling the acquisition of high-resolution images or laser points which may be otherwise not visible or accessible.

In general, MM platforms rely on direct sensor orientation provided by a combination of GNSS, inertial measurement units (IMU), and in some cases odometry information. Depending on the workflow, an integrated sensor orientation or direct geo-referencing is used. In both cases, however, this entails a direct dependency on the accuracy of the position postings obtained by aforementioned instruments, as other independent references are usually missing, e.g. ground control points (GCP).

Whereas GNSS provides an absolute positioning usually every second, IMU and odometer are used to relatively position the platform within this interval. Thus, there are different error sources and types (see Figure 3.1):

1. In case the direct line-of-sight between the receiver and multiple navigation satellites is obstructed, no accurate position fix can be determined - non-line-of-sight (NLOS). In these events, only reflected or no signals are received, IMU/odometer propagate a position with an unknown error relatively until the direct line-of-sight can be restored. In case of a total GNSS outage, position errors increase over time depending on the IMU's gyro bias stability, accelerometer bias, accelerometer scale factor uncertainty, and many other parameters (Novatel, 2014).

2. Another type of position error originates from multipath interferences, where signals reflected from vegetation, the ground, buildings, and other objects are received in combination with signals received directly from the satellite (multiple paths). Multipath effects are considered undesirable, as they affect the time delay function and result in wrong position fixes.

Although both issues are common in urban areas and result in deteriorated position accuracy, their characteristics and error profile are different, and they may occur in parallel. There are multiple mitigation strategies, but no approach is sufficiently effective at detecting and eliminating resulting measurement errors (Groves et al., 2013). Hence, external references have to be introduced to increase the positioning accuracy. 

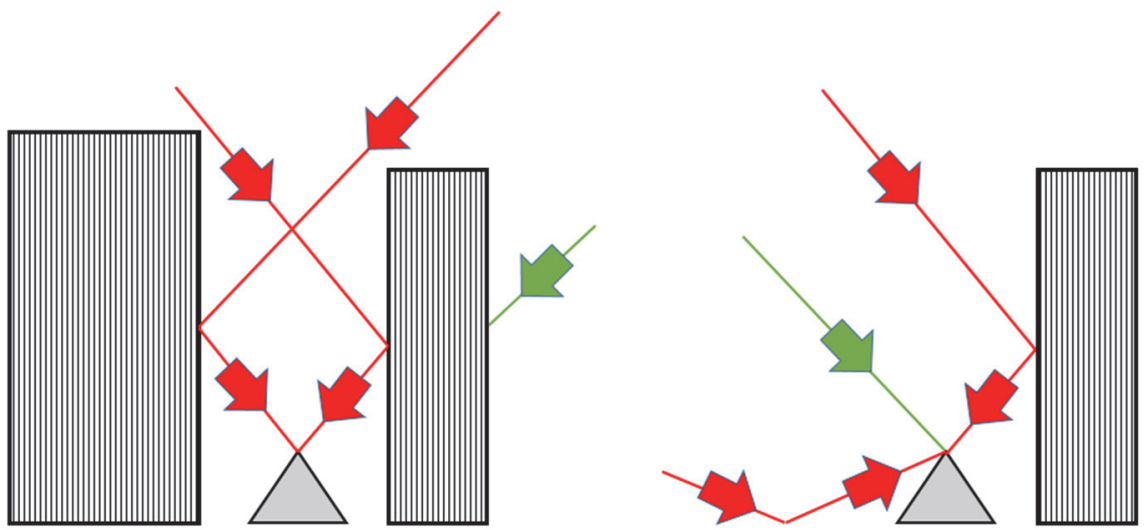

Figure 3.1 Left: Non-line-of-sight problem; Right: Multipath interference problem.

The approach presented in this chapter utilises airborne nadir images as the reference for correct orientation information. With a ground resolution of the aerial data set of approximately $10 \mathrm{~cm}$ (in our test scenarios) and highly accurate correspondences between aerial and MM image data set, the aim of our procedure is to achieve decimetre-level accuracy of the MM data. Another noteworthy property of this method is the possibility for an independent verification of MM data's accuracy without conducting an adjustment of the data itself.

The structure of this chapter is as follows. Section 3.3 discusses related work, thus alternative strategies and an overview on previous work. Section 3.4 comprises the workflow with data preparation and handling as well as the registration strategy. In section 3.5 registration and adjustment results will be presented and discussed.

\subsection{Related Work}

\subsubsection{Alternative strategies}

Predominantly in the field of navigation, shadow matching is a favoured technique where 3D building models are used to estimate which satellites have a direct line-of-sight at a certain moment in time (Groves, 2011). Signals from satellites without a direct line-of-sight are discarded or filtered, respectively. Approaches using shadow matching in conjunction with Kalman filter solutions, Bayesian networks or probabilistic models are real-time capable and reach metre-order accuracy (Gu et al., 2016). These approaches yield promising results, and are of significant interest for real-time applications by being able to achieve lane-level accuracy (Bétaille et al., 2016). Similarly, Strode and Groves (2016) suggest to detect multipath measurements by analysing the 
signal-to-noise ratio. This technique can reduce positioning errors considerably, and the authors propose to exploit shadow-matching techniques as well to reach better reliability and accuracy. The authors, however, state that multipath detection always involves some uncertainty.

In the field of robotics, simultaneous localisation and mapping (SLAM) is an established and well understood technique to navigate a robot through unknown terrain whilst mapping its surroundings. To support these tasks, prior information, such as digital maps or aerial images can be integrated into an estimation procedure. Kümmerle et al. (2011) used building structures and edges directly extracted from aerial images to derive a reference map to support the localisation of an autonomous robot in a SLAM approach. Pink and Stiller (2010) propose a method to automatically create maps containing lanemarkings from aerial images as a prior for camera-based localisation tasks.

Generally, many approaches to support the localisation of autonomous cars rely on lane markings derived from digital maps. Gruyer et al. (2014) employs cameras to detect lane markings, and treats this information as an additional sensor within a Kalman Filter estimation. Similarly, Schindler (2013) combines a laser scanner with a camera to detect lane markings more reliably to enable self-Iocalisation based on Monte-Carlo Localisation. Roh et al. (2016) developed a SLAM-based method integrating publicly available digital maps comprising buildings, roads, and corresponding elevation as an additional measurement complementary to GNSS.

A related idea to SLAM is Visual Odometry (VO), where the relative localisation of a vehicle or platform is determined by tracking image information from an on-board camera or laser scanning system. In conjunction with an IMU, this sensor combination allows for robust positioning, as motion blur can be reduced, feature tracking does not have to be continuous, and even monocular camera systems receive reliable scale estimation. Badino et al. (2013) rely on a stereo camera setup where features are not only tracked to subsequent frames of the camera, but across the entire trajectory which is improving the accuracy of the position estimate. Zhang and Singh (2015) developed a method using a laser scanner/camera combination which initialises the position estimate with VO, but refines the platform's motion utilising the laser scanner for scan matching. Gupta et al. (2016) combine digital map information with VO to localise a vehicle without any GNSS positioning, and achieve metrerange accuracy. $V O$ is a technology of significant importance for autonomous driving and many different approaches are compared using the KITTI dataset (Geiger et al., 2013).

Other approaches for real-time localisation make use of Wi-Fi signals to mitigate GNSS inaccuracies (Ramakrishnan et al., 2013; Wilson, 2016). 
According to Ramakrishnan et al. (2013), the most practical method is to use a database of access points with their exact locations and convert the respective received signal strength to a distance measurement. Even though Wi-Fi networks are abundant in urban areas, the underlying database could have wrong entries, and Wi-Fi signal strength can strongly fluctuate due to attenuation. Moreover, the best positioning possible is in the metre-range.

All methods described above are designed to work in real-time, or support realtime localisation as such, entailing a different goal than our approach, as the accuracy of the data can be considered a means to an end. Contrary to these approaches are those which aim to fix the acquired data, thus offline or postprocessing renders feasible.

Shan et al. (2014) propose a fully automatic pipeline to accurately georeference 3D models based on ground images using aerial images as a reference. The approach registers synthesised views of ground imagery created from dense depth maps with aerial oblique images. This technique supports overcoming strong variations in perspective and scale. The authors report a success rate of about $70 \%$. The approach, however, requires a dense point cloud, which is expensive to compute, and does not involve visibility hypotheses, which may prove impractical in particular in an urban setting.

Ji et al. (2015) propose a method to correct the orientation of MM images with support of aerial images and particle filtering. To this end, a probabilistic location model utilising geometric and radiometric constraints for matching and localisation has been designed. However, a Markov Chain approach has been chosen where just the last position is incorporated, thus omitting one correct measurement may lead to the deviation from the correct trajectory. Moreover, the procedure assumes a certain GNSS error to simplify the transformation model.

Jaud et al. (2013) developed a technique to georeferenced mobile radar data using image-to-image matching based on a Fourier-Mellin transform. This procedure relies on image processing methods, such as colour thresholding, edge extraction, and filtering to register the mobile radar images with an aerial ortho image. As a global registration strategy was selected, good local accuracy cannot be achieved.

Javanmardi et al. (2017) present an approach to correct mobile laser scanning (MLS) data by employing aerial images. Comparable to our procedure, their method predominantly relies on the registration of road markings. In order to achieve a reliable registration result, aerial laser scanning data as well as digital maps were used to prepare the MLS data accordingly. Buildings, vegetation, and other objects are masked prior to the extraction of road markings. Although high planimetric accuracies in the low decimetre-range were 
reported, the procedure requires additional data (aerial laser scan data \& digital maps) and the MLS data is adjusted in a patch-based manner utilising a 2D transformation. The latter may impose a limitation on the accuracy, as the adjustment has a limited number of degrees of freedom and is based on groups of scan lines instead of the trajectory itself.

Cavegn et al. (2016) compare direct and image-based georeferencing techniques for MM data in GNSS-denied areas. In the latter case, external georeferencing has been provided by surveyed GCPs in conjunction with a bundle adjustment to orient the images accordingly. This accords with the standard photogrammetric procedure to improve image orientation. Using GCPs is common practice to increase the accuracy of MM data (Han and Lo, 2016; Hofmann and Brenner, 2016), but is comparatively expensive and labour-intensive.

\subsubsection{Previous work}

One part of this research project carries out updating the positioning of Mobile Laser Scanning platforms (Hussnain et al., 2016), whereas this part focusses on MM imaging data. Since our approach is utilising aerial images as the source of georeferencing, first endeavours have been undertaken with respect to the registration strategy (Jende et al., 2016a). It has been found, that low-level tie feature correspondences, especially corner points, yield the most useful features for a successful registration with aerial images. This is due to the fact, that not just the perspective, but also scale and rotation of the MM data set can be adjusted to the properties of the aerial imagery, and thus reducing unnecessary degrees of freedom. By ortho-projecting MM images to the ground similar to Inverse Perspective Mapping (Muad et al., 2004), the resemblance to the aerial imagery can be increased.

Even though the images share similarities, the original perspective and their recording date is still different entailing divergent image content. To support the registration of both data sets, MM orientation parameters can be used. Although their accuracy depends on the influence of GNSS errors, they enable restricting the search for correspondences to a geometric threshold instead of sifting through the entire image. In general, the most prominent features visible in both, the aerial and the MM data set, are road markings which can be repeated within the geometric search window. This may lead to wrong correspondences due to an ambiguous appearance. In order to obviate wrong feature correspondences, an initial, but reliable transformation between both images has to be found. This technique has been first introduced in Jende et al. (2017) and showed promising results. 
The contributions in this chapter extend this technique to further its robustness and accuracy as well as presents the entire workflow from the raw data set to correspondences which can be used within an adjustment solution.

\subsection{Methodology of the registration}

\subsubsection{Overview}

This section comprises the workflow and its methodology in detail (see Figure 3.2). The processing is set up to work in an automatic fashion, and processes MM images according to their location in the platform's trajectory - from start to finish. Even though, the procedure is designed for post-processing, this architecture potentially allows for real-time processing as well. On the other hand, it could be extended towards a parallel design to reduce computational time. Starting from the raw MM data, the aerial nadir data and their respective meta data, the final output are correspondences between the data sets in image as well as in object space. This property is a requirement by the adjustment procedure, which had to be outsourced, as our own approach is currently under development. The registration of the data relies on an array of techniques to overcome the differences between both image data sets. The details on the specific components are discussed in the next sections. 


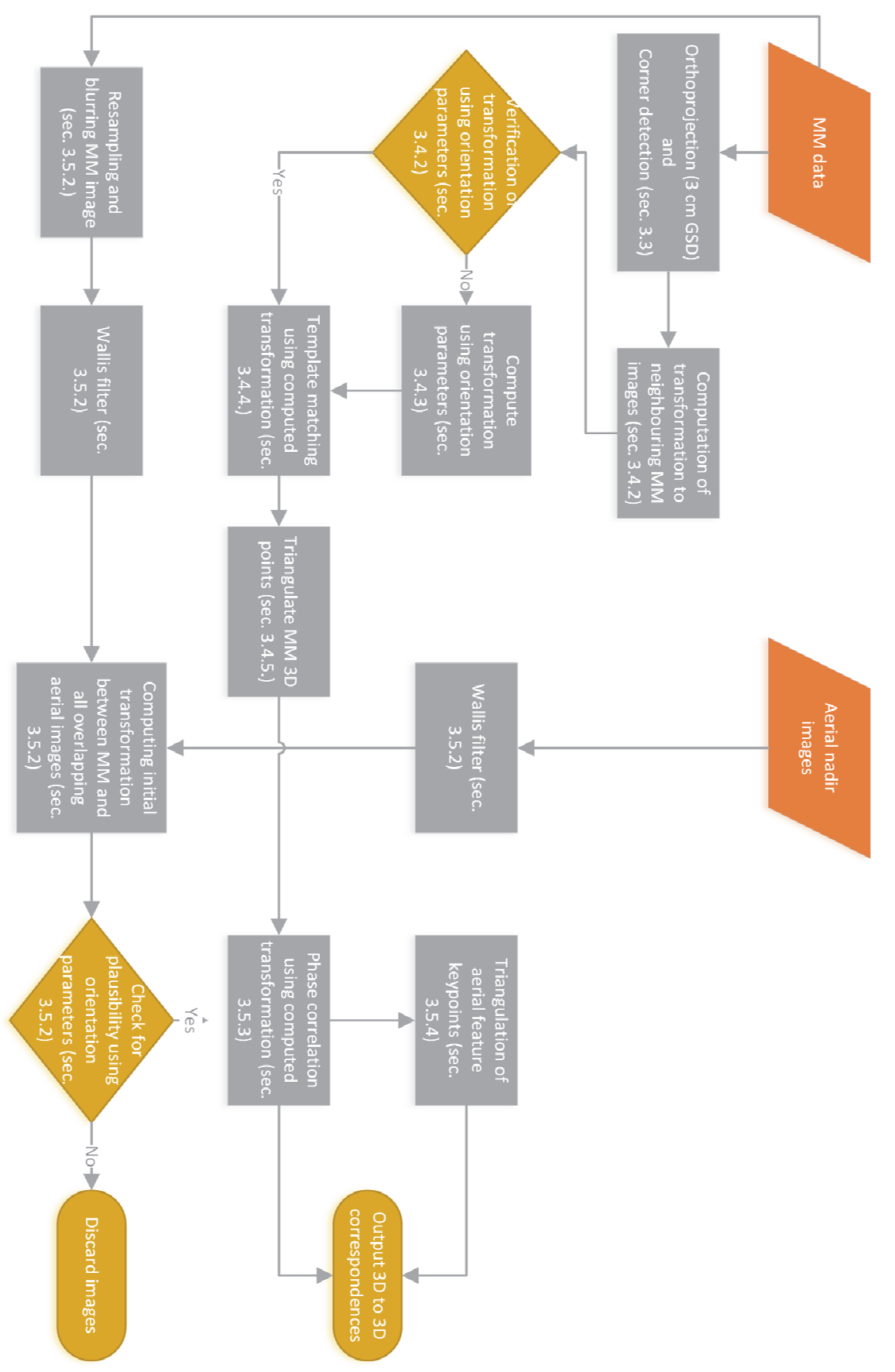

Figure 3.2 Design of the registration procedure [reference to respective section in brackets]. 


\subsubsection{Processing along the trajectory}

MM data is acquired in a sequential manner. Usually, the operator of a MM platform records data according to a predefined route. Consequently, MM images are chronologically ordered and shape a trajectory. Due to GNSS and IMU-based positioning, adjacent MM images share a similar error pattern due to spatial correlation. Regardless of the absolute error of the platform, adjacent recording locations retain a high relative accuracy. Tactical-grade IMUs ${ }^{18} \mathrm{drift}$ effects are insignificant over short distances, and filtering techniques (e.g. Kalman or particle filter) used for computing the trajectory smoothen position fixes.

Hence, the registration procedure is designed in a sequential fashion to ideally benefit from the high relative accuracy along the trajectory. This simplifies the registration procedure of $M M$ images while enabling its verification by $M M$ orientation parameters. MM images are registered to yield observations in object space as well as to link the images for the adjustment by employing relative constraints.

\subsubsection{Ortho-projection / Inverse perspective mapping}

Aerial nadir cameras capture scenes from far above in a vertical perspective unlike MM platforms which record a 360 by 180 degrees panoramic image from a terrestrial viewpoint. Since the process relies on aerial images as the source of correct orientation parameters, and $\mathrm{MM}$ data has a higher resolution, $\mathrm{MM}$ images are transformed to correspond to the aerial reference. MM images are encoded in an equirectangular projection, i.e. a sphere is mapped onto a raster grid with $360 * 180$ degrees with a pixel representing 0.075 degrees. Thus, a pixel translates into a measurement of an azimuthal and a polar angle.

MM images can be ortho-projected using various methods. The technique that is used in our case utilises a horizontal plane (30 m diagonally) defined in space centred at the perpendicular foot of the camera location. By discretising the plane's world coordinates into a pixel grid with an arbitrarily set pixel spacing, the corresponding MM image's spherical coordinates can be related to the plane by a back-projection mechanic (see Figure 3.3). This entails that an interpolation of grey values in the ortho-projected image is not required, since every ray from the ground plane to the camera's projection centre will always pierce the panoramic image plane. Certainly, multiple assignments of the same panoramic image's pixel in the ortho-projected image occur depending on the distance to the projection centre. If the procedure were designed the other way around - a forward projection from the panoramic image onto the ground

\footnotetext{
${ }^{18}$ Accelerometer bias of about $1 \mathrm{mg}$ and gyro bias of 0.75 degrees/h
} 
plane - data gaps would occur the larger the distance from the ground point to the projection centre is.

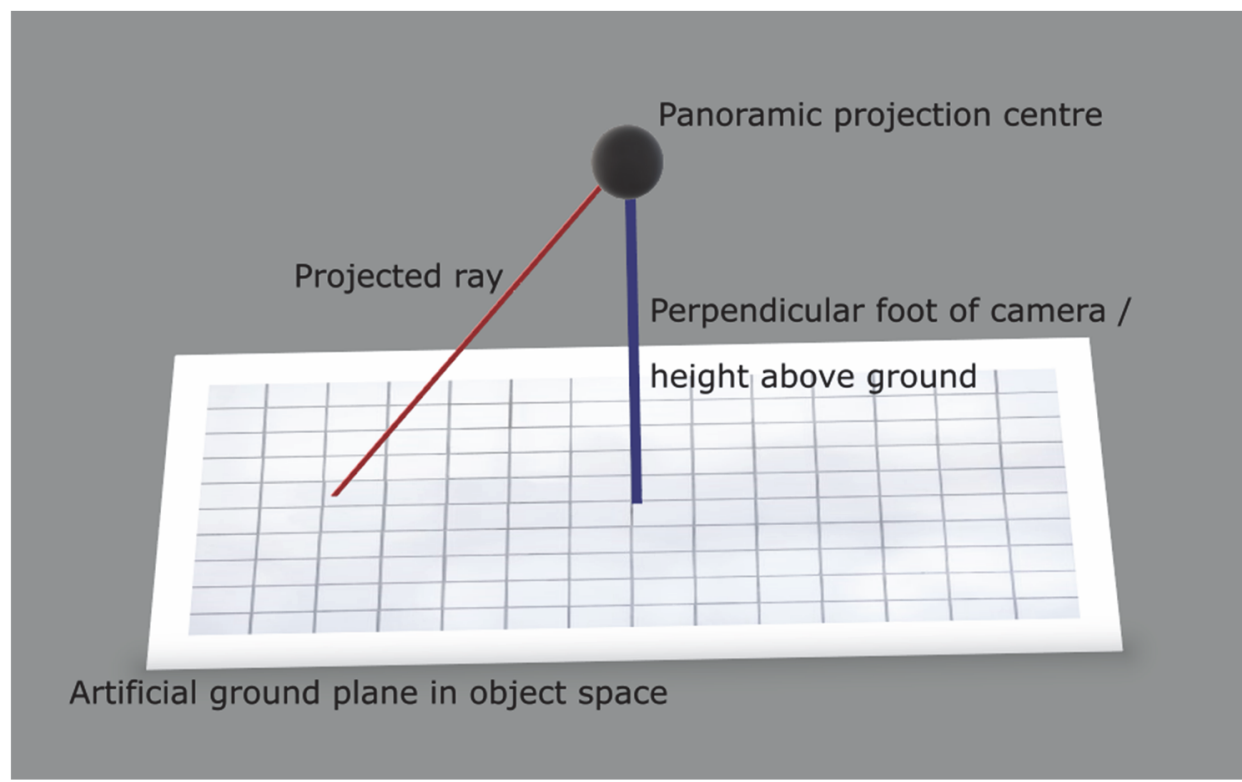

Figure 3.3 Principle of ortho-projection.

As only points on the ground up to a defined distance to the camera location are projected into the image, the resulting ortho-image represents a perspective on the scene resembling an aerial nadir view. Objects, such as cars, lamp posts or traffic lights can obstruct the visibility to the ground and can thus hamper the registration process with the aerial images. Additionally, the projection allows for mapping the image content to an arbitrary orientation, e.g. to align with the mapping reference frame. This simplifies the registration with other images, be it MM or airborne. For instance, as the aerial images are oriented in flight direction, the MM patch is rotated to align with their respective yaw orientation. For the registration of $M M$ images along the trajectory (see 3.4.4), the resolution of the ortho-projection images is set to $3 \mathrm{~cm}$ to benefit from a more image detail. The registration procedure between aerial nadir and $\mathrm{MM}$ images requires a lower resolution for a better result. Hence, the MM are ortho-projected again for this step, but with a resolution of $10 \mathrm{~cm}$.

Another noteworthy property is masking the bonnet of the car (see Figure 3.4). The MM car's roof is masked by default. As the MM images' vertical centre is always oriented north, the platform's bonnet may be located arbitrarily in the raw panoramic image. By determining the orientation of the platform, the bonnet can be masked in the ortho-projection procedure to reduce the risk of false correspondences. 
The ortho-projected image may entail distortions due to the hypothesis of a horizontal ground surface. To avoid potential errors, ortho-projected images are used for image registration only, and actual measurements (i.e. 3D triangulation) are conducted in the original spherical geometry after applying the inverse of the projection to identified correspondences.
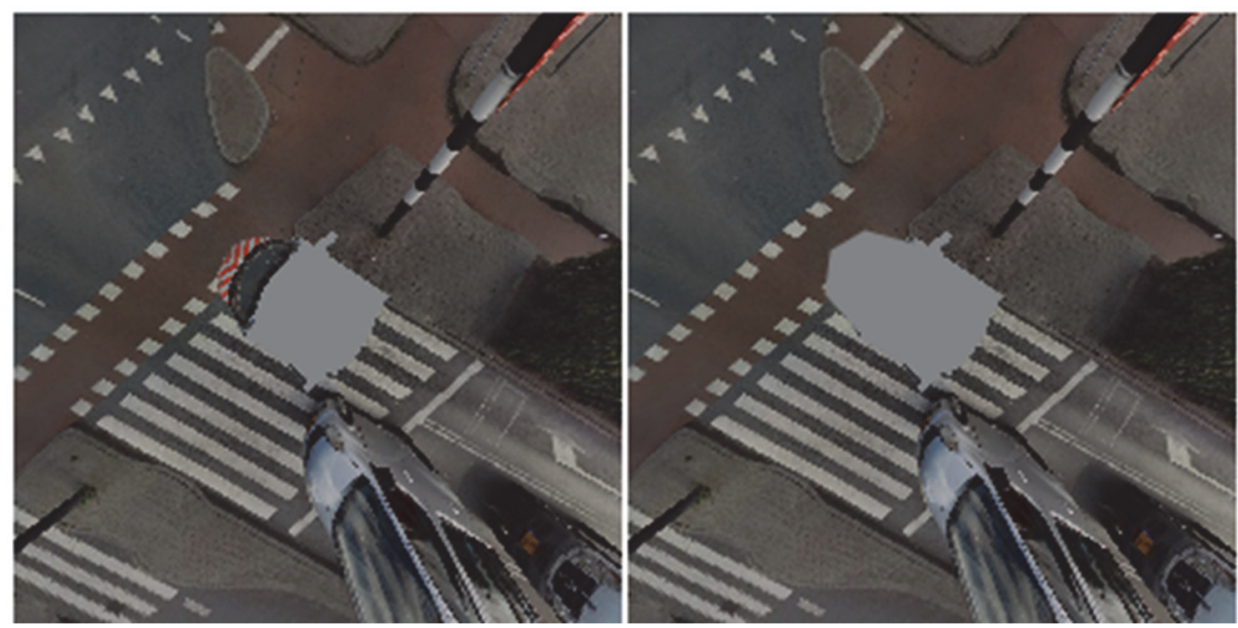

Figure 3.4 Ortho-projected MM image with masked bonnet. Please note the highly distorted silver car in the bottom half of the image or the traffic light's post. These distortions are unfavourable side effects of the ortho-projection.

\subsubsection{Matching panoramic images to obtain 3D points}

\subsubsection{Overview}

Following the projection of MM images to an artificial ground plane, adjacent MM images are registered with each other. Depending on the set properties of the procedure, either two or three adjacent MM images are matched. In the latter case, registered features from two MM images are tracked and matched with a third MM image.

The registration of adjacent MM images is performed in a two-step procedure. The first step is finding the appropriate transformation relating one image to another, the second step is detecting and matching corner features across MM images. Corner features are favoured over e.g. blob features, as they are more likely to be identified in the aerial images as well.

After the registration of the respective MM images, features are triangulated to obtain their object coordinate. This in turn is required by the adjustment procedure which relies on 3D-to-3D correspondences with the aerial reference data set. Thus, all common features identified in the MM images are to be sought for in the aerial images as well. 


\subsubsection{Initial transformation and its verification}

In order to retrieve a reliable registration of MM images, relative orientation parameters could be used to support the matching task. The relative orientation between two adjacent MM images is accurate, however, a projection to an artificial ground plane may affect this accuracy in particular if the surface is not exactly flat. To this end, a transformation between adjacent ortho-projected MM images is computed instead.

Eventually, corner points in the MM images have to be detected and matched. Yet, these features are not as abundant and may not be as well distributed as blob features are. Thus, KAZE features (Alcantarilla et al., 2012) are used for determining the initial transformation between adjacent MM images. Our previous efforts with respect to finding a suitable feature detector/descriptor combination have been published in Jende et al. (2016a).

The MM images share the same rotation as well as to some extent the same scale - depending on the smoothness of the actual ground surface. Hence, the KAZE detector can operate using an upright-constraint and one octave only. Due to the small temporal offset and since the same camera was employed while having a large overlap between both images, illumination and contrast differences are negligible.

After KAZE feature correspondences have been found, RANSAC is used to filter outliers as well as compute a homography transformation between the MM images. In order to verify the computed transformation, the image's centre of the first MM image is projected into the second one. By computing the distance from the projected first image centre to the second image centre, it can be compared to the actual metric distance of the image poses. Hence, an empiric threshold set to $30 \mathrm{~cm}$ determines whether a transformation is valid.

The advantage of that procedure is mapping in discrete pixel coordinates instead of utilising orientation parameters which in turn allows for a more precise transformation - also if the ground is not exactly flat and the orthoprojected images are thus distorted. Only if the ground surface is strongly slanted (i.e. slope of more than 5 degrees), this procedure will likely fail.

\subsubsection{Alternative solution based on orientation parameters}

Although finding a transformation based on KAZE features is the more accurate technique and works to some extent on distorted images, it may fail if there are not enough features, too many mismatches occur, or RANSAC converges to a wrong solution. Since the transformation can be verified and thus be proven wrong, orientation parameters also serve as an alternative solution to registering $\mathrm{MM}$ images. 
If this is the case, MM image are registered with support of their orientation parameters. To this end, corner points are identified in one ortho-projected MM image and projected into the other. In this case, the features' pixel coordinates can be simply translated to world coordinates without resection, as they are located on the artificial ground plane defined earlier for the ortho-projection.

\subsubsection{Matching with corner features and templates}

The actual registration of MM images relies on corner features at subpixel accuracy (Förstner and Gülch, 1987) and template matching. As mentioned earlier, these features are re-used for the registration with the aerial data, but can be also tracked to other MM images.

Corner features are only detected in one ortho-projected MM image and projected into the second image. Depending on either of the two methods described earlier, the projection is performed using the transformation based on KAZE-matching or utilising MM orientation parameters. If the latter method is applied, corner features are detected and directly projected into the second MM image.

Template matching is used for locating the projected corner point in the second MM image at the same location than in the first MM image. Since the images share the same rotation, approximately the same scale, and similar illumination and contrast properties, it is mostly varying image content or occlusions due to small temporal offsets or differences in the image poses, which could hamper a successful registration.

Lastly, the registration is verified again similarly to the approach described earlier finding the transformation using KAZE.

\subsubsection{Triangulation of image observations}

Corner points in the ortho-projected MM image can be translated into equirectangular coordinates using the inverse of the ortho-projection technique. Thus, they coincide with the original panoramic perspective of the MM images. After converting these coordinates into directional vectors and relating them to the individual camera positions, these image observations can be triangulated to obtain their respective object coordinate. If feature points are visible in three or more images, the accuracy of the reconstructed point in object space is higher. However, in order to retain a higher number of observations, two-view matches are accepted as well. 


\subsubsection{Matching panoramic images with aerial reference data}

\subsubsection{Overview}

Subsequently to registering MM images along the trajectory of the platform, direct correspondences to the aerial nadir images need to be found for enabling an adjustment of the MM images. Corner features that have been identified, matched, and triangulated in the previous steps are re-used for this task.

Hence, respective MM image recording locations are projected in every overlapping aerial image. Since the recording location may be inaccurate due to a difficult positioning scenario, a cropping of an aerial image counterpart has to account for this issue by a larger extent of the resulting patch. This will ensure that correspondences can be found, as the aerial image patch will overlap the MM image to the extent of the expected localisation error of the MM platform. Subsequently, a transformation based on the respective orientation parameters for every related aerial and MM image patch is computed.

The corner features belonging to a respective MM image are then projected into each overlapping aerial image using the respective transformation. Similar to the template-based registration between MM images, an area-based method is used to locate the corner feature precisely in the aerial image. As the aerial and MM image may differ in their radiometric properties, the correlation value is derived in the frequency-domain to achieve a better invariance of the registration. After identifying correspondences with at least two aerial images, these points can be triangulated using the aerial images' orientation elements. As a result, a point in object space is observed by at least two MM and two aerial images.

\subsubsection{Finding a transformation between $M M$ and aerial image patch}

Matching images with non-standard geometries is a challenging task. As mentioned earlier, MM images are thus projected to an artificial ground plane to increase the resemblance with aerial nadir data. The resolution of the projected MM images is set to match the aerial images' resolution, i.e. to a resolution of $10 \mathrm{~cm}$. Rotation, scale and perspective between both images is thus similar. However, the original image perspective entails differing image content, and the images may vary strongly in illumination and contrast due to different camera properties and acquisition time.

Subsequently to the retrieval of overlapping aerial image patches, a transformation between the respective MM and all individual aerial image patches is computed. This essential step increases the robustness of the entire procedure. Since mostly street scenes are to be registered, road markings are 
prominent features and ideal for this purpose. Zebra crossings and other repetitive patterns, however, introduce ambiguity to the description of feature points regardless of the algorithm - be it an area-based or a feature-based approach.

An intuitive idea is exploiting the uniqueness of certain features to find a few, but reliable correspondences, which are then used to retrieve a transformation between both images. As MM orientation parameters can be used, feature ambiguity is not subject to the entire image patch rather than search windows with the size of the expected localisation error. Thorough experiments have been carried out to understand the feasibility of this approach (Jende et al., 2016b). Even though this technique can work under certain conditions, employed data sets vary in too many aspects in order to detect the same unique features in a reliable and reproducible fashion. In particular, the original perspective entailing different image content leads to a different classification of uniqueness.

In contrast, using a highly constrained feature matching approach based on KAZE features, Wallis filtering (Wallis, 1976), and plausibility checks has shown promising results. The motivation to performing a new iteration of feature detection and description using KAZE is to achieve full independence for finding a reliable transformation between every MM and aerial image patch - a condition which cannot be met with existing corner features. To this end, a Wallis filter is applied to both, MM and aerial image patch. A Wallis filter enhances image contrast locally while reducing the overall contrast in the image. Especially in urban scenarios, shadows cast by buildings affect feature matching, as illumination changes within one image are unlikely to be repeated in the other image. Hence, filtering the images reduces illumination differences within the images, and allows for a higher resemblance between the MM and aerial image (see Figure 3.5). The activation threshold of the KAZE detector is set to account for the differences between MM and aerial image, thus two different instances of the detector are initialised. The KAZE descriptor, however, adheres to one parameter set for both image types with an extended description of 128 bytes per keypoint, an upright-only description to disable rotational invariance, and a single octave, as the images share a similar scale.

Keypoints are first detected in the MM image. Since the artificial ground plane is known in object space, detected MM keypoints are converted into world coordinates and projected into the aerial image. To account for potentially imprecise MM orientation parameters, a window of the expected maximum localisation error [in px] is defined at the location of a back-projected keypoint. Thus, the corresponding keypoint in the aerial image is expected to be within the defined window. Within this window, the second instance of the keypoint detector - parametrised for aerial imagery - is used to identify salient features. 
Subsequently, a one-to-many descriptor matching is performed between the projected MM keypoint and the entire set of identified features within the window.
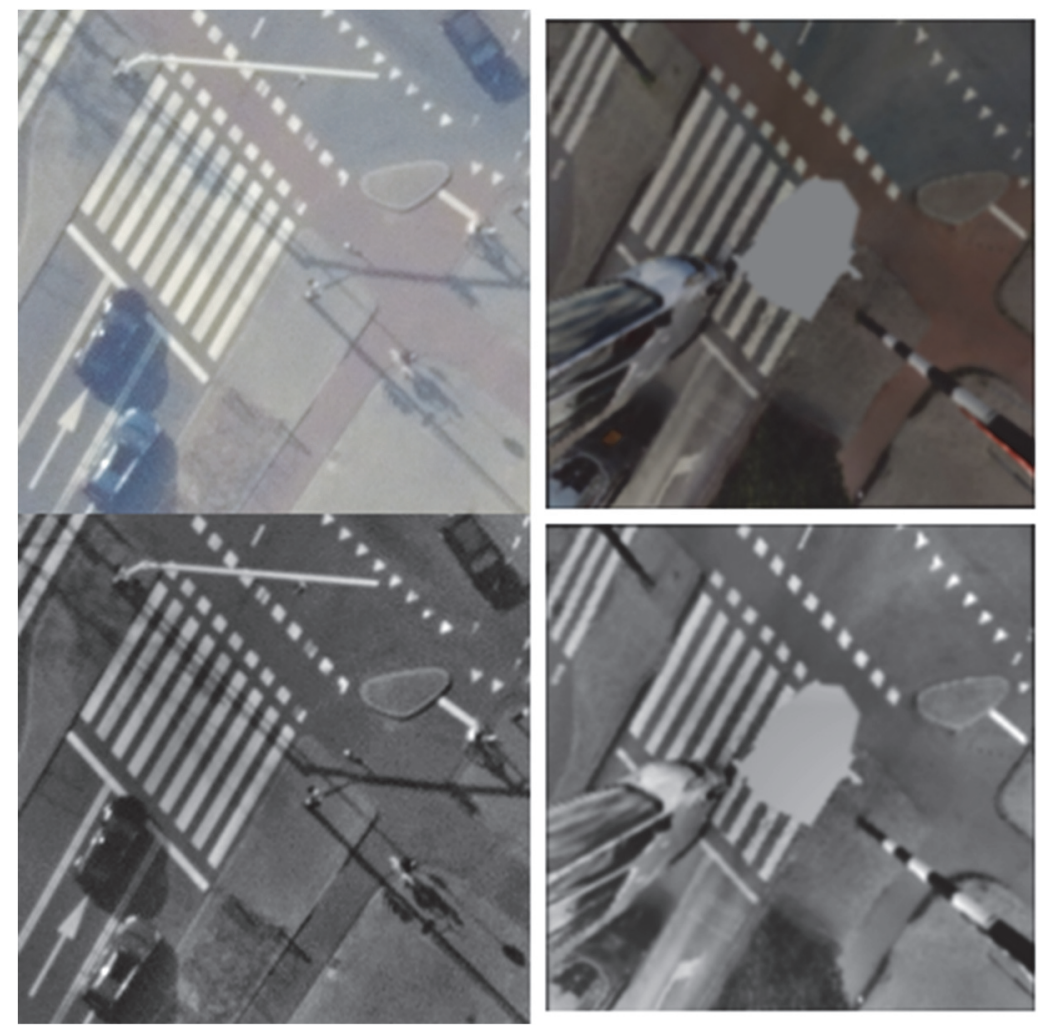

Figure 3.5 Aerial and MM image patches; bottom: Aerial and MM image patch after Wallis filtering - the output is always a greyscale image.

The identified correspondence in the aerial image is then related to the image patch coordinate frame. This procedure is repeated for all MM keypoints. After the registration, a similarity transformation is fit to the set of correspondences using RANSAC. This transformation is checked for plausibility by projecting the MM image's centre coordinate into the aerial image. If the offset is larger than the expected localisation error, the transformation is presumably wrong, and will thus be discarded.

This transformation is computed for each aerial image patch overlapping a respective $\mathrm{MM}$ image. As an additional option, the transformation can be saved and re-used for adjacent $\mathrm{MM}$ images. Since the relative orientation along the trajectory is accurate, the transformation parameters do not change significantly between neighbouring images. 


\subsubsection{Accurate registration of MM and aerial image}

Earlier in the process, corner features have been identified in MM images by using the Förstner detector. These corner points can now be projected into an aerial image patch if a transformation could be computed and passed the verification process.

As the accuracy of the computed transformation may vary due to distortions in the ortho-projected MM image or imprecise feature correspondences, the registration process is based on template matching. To find the exact corresponding point in the aerial image, a template in the MM image as well as a window in the aerial image is defined.

Both image regions are compared in their frequency domain by phase correlation. Although edges are per se illumination-invariant or state-of-theart feature matching techniques employ normalisation techniques, strong variations in illumination and contrast caused by e.g. a changing origin of the light source can affect the local grey value distribution in the image. Since feature-based approaches rely on the consistency of this representation, descriptions based on grey values may fail under these circumstances (Wan et al., 2015).
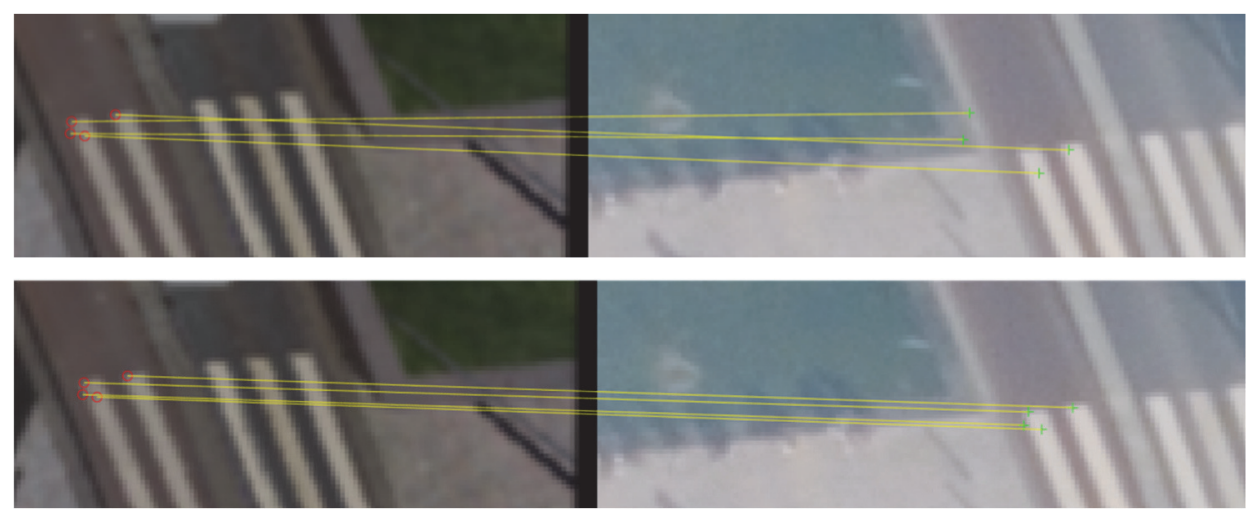

Figure 3.6 Top: Exemplary registration results: Normalised cross-correlation template registration of a MM ortho-image (left) and an aerial image (right). Bottom: Phasecorrelation registration. Please note: this is a result without outlier removal.

Figure 3.6 shows a registration example of phase correlation compared to normalised cross-correlation. The example depicts a difficult matching scenario which is typical in our case when coping with two different sensors, perspectives, and acquisition times. Normalised cross-correlation does not perform that well under these conditions. Phase correlation, however, is invariant to noise and strong illumination differences. The cross-power spectrum of two images in their corresponding Fourier transform $F_{1}$ and $F_{2}$ is defined as 


$$
\frac{F_{1}(u, v) F_{2}^{*}(u, v)}{\left|F_{1}(u, v) F_{2}^{*}(u, v)\right|}=Q(u, v)
$$

with $F_{2}{ }^{*}$ being the complex conjugate of $F_{2}$ (Reddy and Chatterji, 1996). The interference fringes in the cross-power spectrum retain their orientation and density regardless of illumination changes, but might be shifted vertically and/or horizontally. By computing the inverse of the cross-power spectrum, a correlation matrix is obtained whose peak determines the displacement between the two images. Additionally, the peak's subpixel location is determined by fitting a second order polynomial to the peak's neighbourhood. Since distortion and other artefacts are locally negligible, only the translation elements are identified to locate the MM corner point precisely in the aerial image.

\subsubsection{Optimising point locations across all aerial images and triangulation}

Aerial images do not have to be registered again, as the correspondences between a specific MM image and an arbitrary number of overlapping aerial images originate from the same set of corner points, and have been thus registered indirectly with each other.

However, their locations across overlapping aerial images need to be verified. Even though phase correlation returns an accurate registration result, local image properties may affect the accuracy of image observations. This is an essential step, as deviations from the exact location will have an impact on the triangulation accuracy.

To this end, point locations are verified using a multi-image template matching approach. If a MM corner point can be identified in at least two aerial images, its object coordinate can be determined by triangulation. That way, the observation in object space is independent from an uncertain MM localisation quality, and MM and aerial data is only linked by image observations. The triangulation itself is based on a least-squares estimation, which returns a covariance matrix to weight an observation in the bundle adjustment.

\subsection{Results and discussion}

This section discusses the results achieved by the registration procedure, and presents exemplary results of the outsourced adjustment procedure to demonstrate the feasibility of our approach. Currently, our own adjustment method is under development and will be covered in detail in a future publication. 
This approach has been developed with an actual application in the background using real world data. The city centre of Rotterdam in the Netherlands has been selected as our primary test area, as it features high-rise buildings, urban canyons, and thus depicts the ideal scenario for localisation problems of terrestrial mobile mapping. An artificial offset in the horizontal dimension to the MM data set had to be introduced, as the original accuracy is not subject to be disclosed due to proprietary knowledge of the data provider. Yet, in order to ascertain the feasibility of the approach, the accuracy of the adjusted MM data will be compared to surveyed GCPs.

\subsubsection{Test data}

\subsubsection{Aerial nadir images}

The aerial data set consists of 15 individual images, and offers a nadir perspective only with a forward overlap of $60 \%$ and $40 \%$ across-track. Since the city of Rotterdam is in close vicinity to the international airport of Amsterdam, the flying altitude for airplanes is strictly regulated. Thus, the data used in this study has been acquired at an altitude of about 4500 metres over ground. Since Rotterdam is located at about sea level, this high altitude leads to an unfavourable geometry with glancing intersections, and thus to inaccurate measurements particularly in height. These effects, however, are not that pronounced with respect to horizontal measurements.

\subsubsection{Mobile mapping images}

As far as MM data is concerned, three test areas depicting major roads as well as byroads in the city of Rotterdam have been selected (Figure 3.7). MM images are captured every 5 metres along the trajectory, and are available in an equirectangular projection with respective meta data, such as camera location, camera height over ground, and acquisition date and time. As stated earlier, MM images' positions have been artificially deteriorated. This has been done by introducing a systematic as well as a random error $(80 \mathrm{~cm}$ and about 10 $\mathrm{cm}$, respectively) to the original positions of the platform. That mechanic simulates considerable GNSS positioning problems. Each test area consists of multiple trajectories, which are interrupted or may overlap. As mentioned earlier, the registration procedure takes the acquisition sequence into account in order to utilise a high relative orientation. 


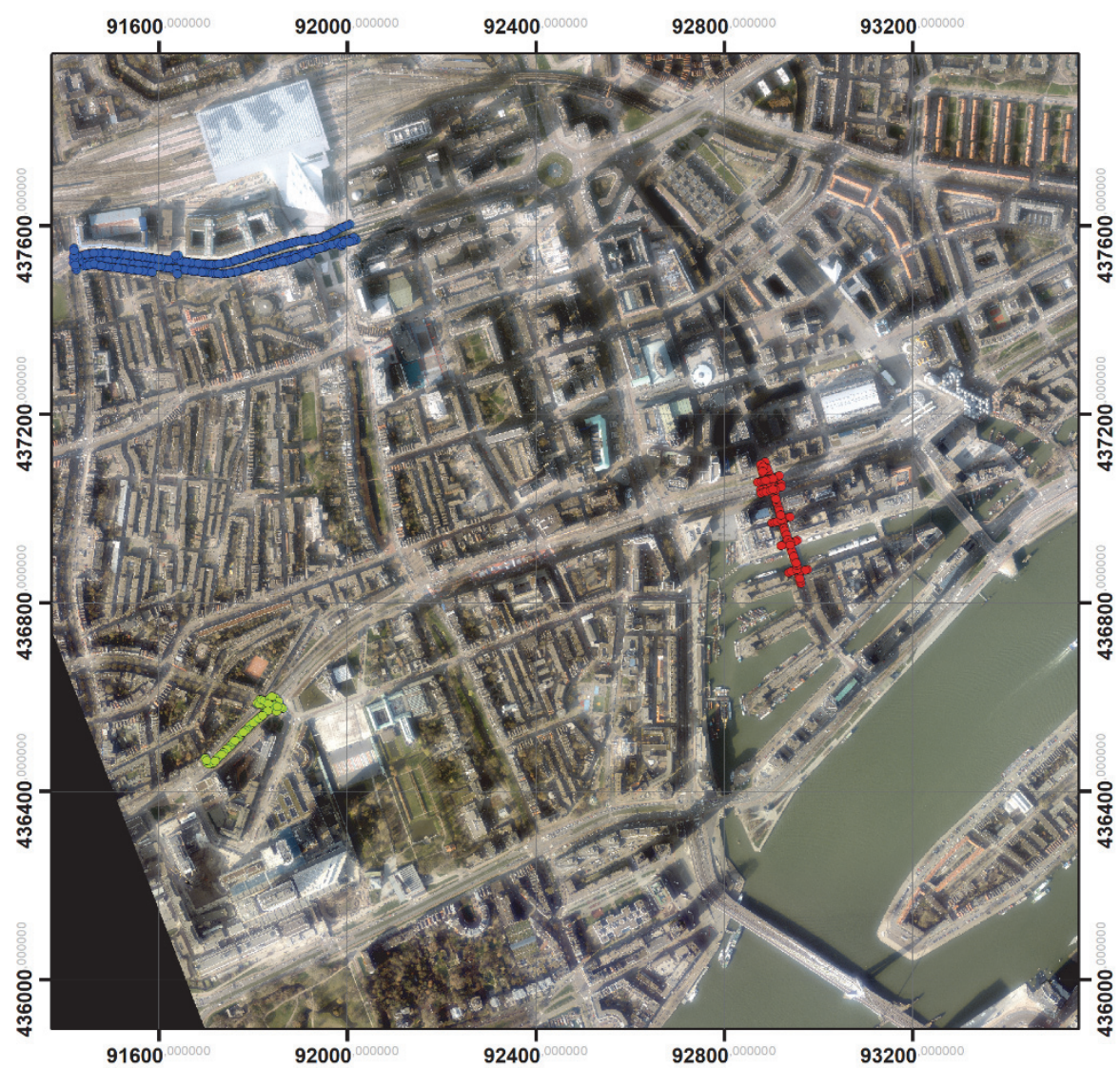

Figure 3.7 Mobile mapping test areas in Rotterdam with coordinate axes in the RD New system. $[$ Red $=$ Area 1; Green = Area 2; Blue = Area 3]

\subsubsection{Registration results}

In the following section, registration results will be discussed. Table 3-1 gives an overview on the three trajectories, which serve as an example for the registration and adjustment procedure. The overall inlier ratio is fairly high. Remaining outliers can be removed during the adjustment procedure by e.g. exploiting differing back-projection errors. Both entries, number of tie points with individual aerial images and average number of aerial images matched with MM image, give an idea how many 3D tie points can be derived, and contribute to the adjustment. 
Table 3-1 Overview of registration results of test areas in Rotterdam.

\begin{tabular}{lccc} 
Area & Area 1 & Area 2 & Area3 \\
\hline \hline $\begin{array}{l}\text { Number of MM images } \\
\text { Number of MM images with direct } \\
\text { correspondences to aerial images }\end{array}$ & 116 & 69 & 317 \\
$\begin{array}{l}\text { Number of tie points } \\
\text { with individual aerial images }\end{array}$ & 28 & 22 & 47 \\
$\begin{array}{l}\text { Number of correct } \\
\text { tie points }\end{array}$ & 928 & 687 & 1583 \\
$\begin{array}{l}\text { Inlier ratio compared to total } \\
\text { number of corr. }\end{array}$ & 909 & 682 & 1538 \\
$\begin{array}{l}\text { Average number of aerial images } \\
\text { matched with MM image }\end{array}$ & $97,95 \%$ & $99,27 \%$ & $97,16 \%$ \\
\hline
\end{tabular}

\subsubsection{Distribution of correspondences}

About 15 to $25 \%$ of the MM images within a trajectory have direct correspondences to the aerial reference data. As mentioned earlier, the registration procedure relies on road markings and other distinct ground features, which are sparse or non-existent in small alleyways. Consequently, most of the tie points supporting the adjustment of MM data will be found around junctions, zebra crossings, or areas with salient road markings. Since junctions are abundant, well and more or less equally distributed in our test area, this limitation of our approach is of minor importance in this case, as relative constraints can propagate an orientation update.

However, there are likely to be settings of sparse suitable features in which our registration and adjustment technique cannot return reliable results. Furthermore, MM images are relatively linked in an adjustment, thus it depends on the location or rather distribution of MM images with direct correspondences in the trajectory, if high accuracy can be achieved. In this chapter, the adjustment serves the purpose to demonstrate the feasibility of our approach. In an upcoming study, the effects of the distribution of MM images with direct correspondences to the aerial reference data will be analysed. 


\subsubsection{Quality of correspondences}

Even though the inlier rate of correspondences between MM and aerial images is high, the accuracy and thus quality of individual correspondences differs. High accuracy translates to the identification of the very same image point (at subpixel accuracy) across all images involved, be it MM to MM image, MM to aerial image, or aerial to aerial image. This is, however, not realistic to ascertain. Thus, correspondences were manually classified as inliers if they represent the same correspondence within a pixel.

There are multiple possible reasons for a variation in accuracy, such as differing image detail, an imprecise transformation (see 3.4.5.2 ) mapping features from one image to another, which is still within the thresholds, or the corresponding point cannot be precisely located in the other image due to limitations of the algorithm.

The first one is the most common reason for deviating feature correspondences. Figure 3.8 depicts the problem of image differences hampering the exact localisation of the corresponding point in the aerial image. To overcome this problem, MM images need to have a higher resemblance to the aerial images. For instance, instead of simulating the optical transfer function by Gaussian blurring, it could be properly derived by leading to a more similar image detail.
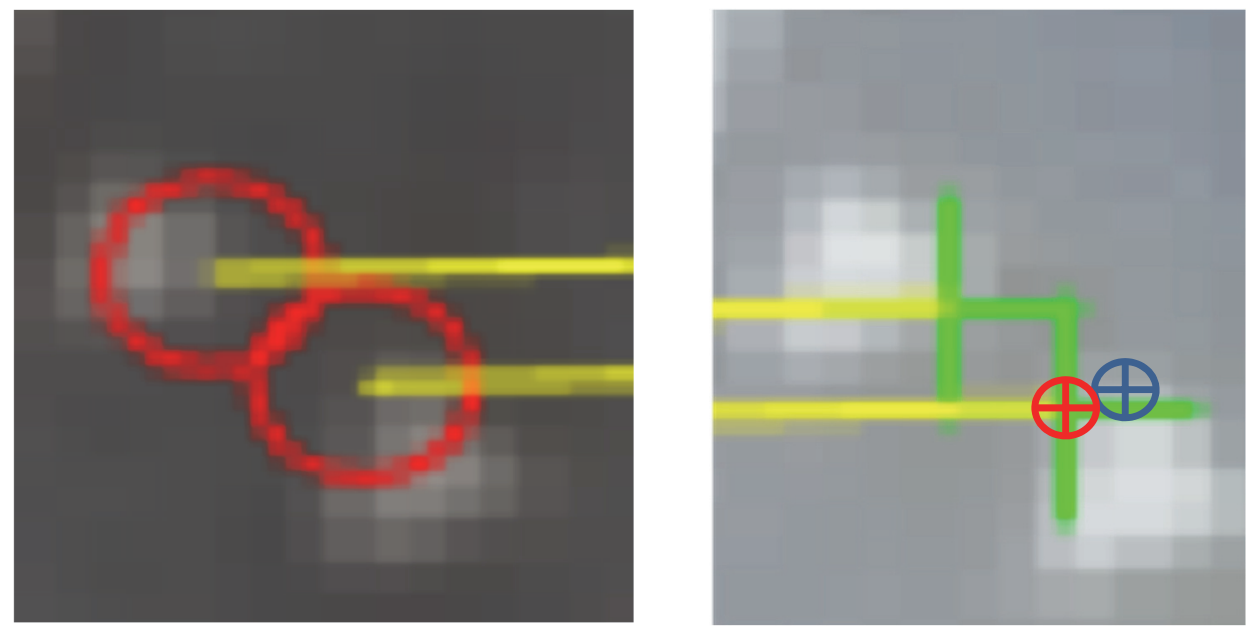

Figure 3.8 Localisation limitations due to differing image properties; Left: MM image, right: Aerial image; Overlay: the red mark indicates the identified correspondence, the blue mark the presumed correct position. Thus, the correspondence has an offset of more than a pixel.

Similarly, correspondences across aerial images are refined by template matching again. In some cases, this may lead to little offsets (see Figure 3.9). 
This is due to the original orientation of the aerial images, as templates are rotated for matching. When correspondences have been identified, their coordinates are rotated into the original orientation again which may lead to rounding errors.
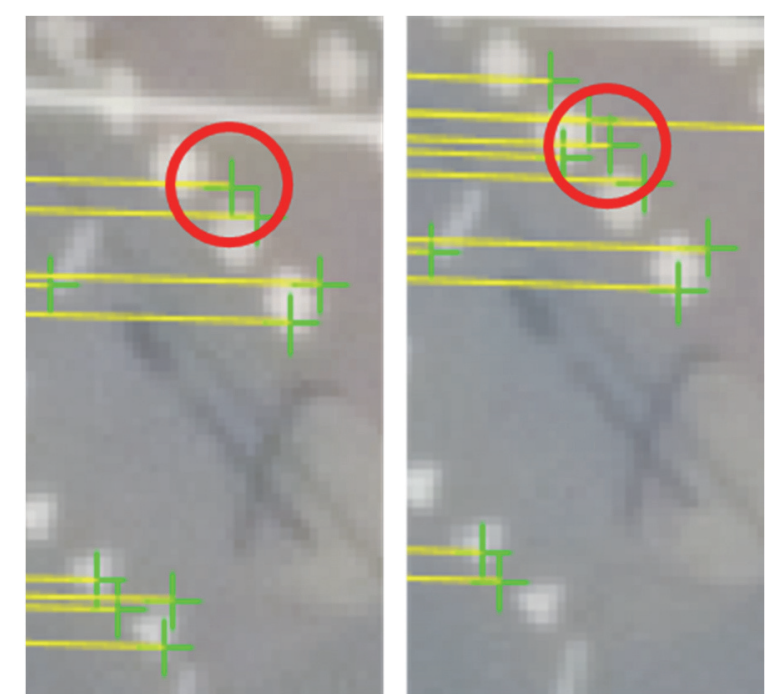

Figure 3.9 Registration result of two different aerial images. Red circle depicts correspondence which is not at the same location with an offset of about a pixel. Remaining correspondences are accurate.

Certainly, MM and aerial images are very different, yet corner features falsely identified at linear segments cannot be determined reliably in the other image (see Figure 3.10).
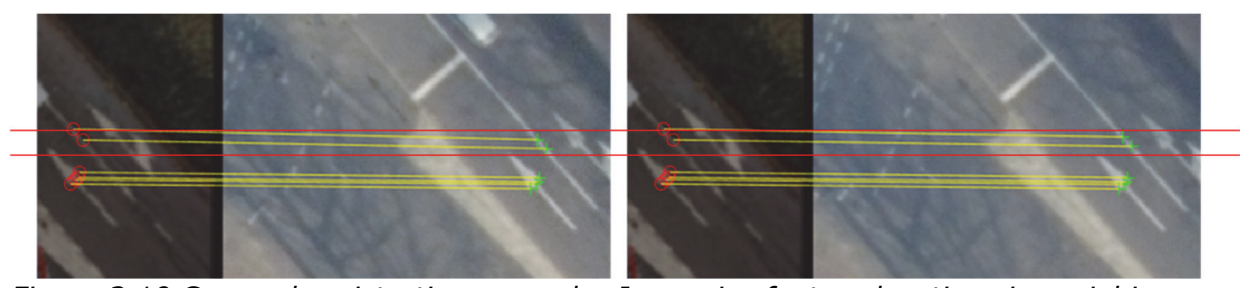

Figure 3.10 Cropped registration example: Imprecise feature locations in aerial images; from left to right: $M M$ image, $1^{\text {st }}$ aerial image, same $M M$ image, $2^{\text {nd }}$ aerial image; Please note: the horizontal red lines in the figure have been manually added to make a comparison easier.

These issues may impact the triangulation accuracy of aerial correspondences in a later stage. In particular in combination with an unfavourable intersection geometry, errors in height can be strongly pronounced. Thus, an outlier removal after the registration phase is recommended, although the accuracy of the identified correspondences is high. 
On the other hand, the registration procedure is able to handle illumination differences and repeated patterns well (Figure 3.11). The approach to find an initial transformation overcomes illumination and contrast differences by Wallis filtering, a different parameter set for each image type, and plausibility checks. This enables a mapping of the entire corner feature data set from the MM into the aerial image while finding correspondences at subpixel accuracy using a phase-correlation area-based matching.

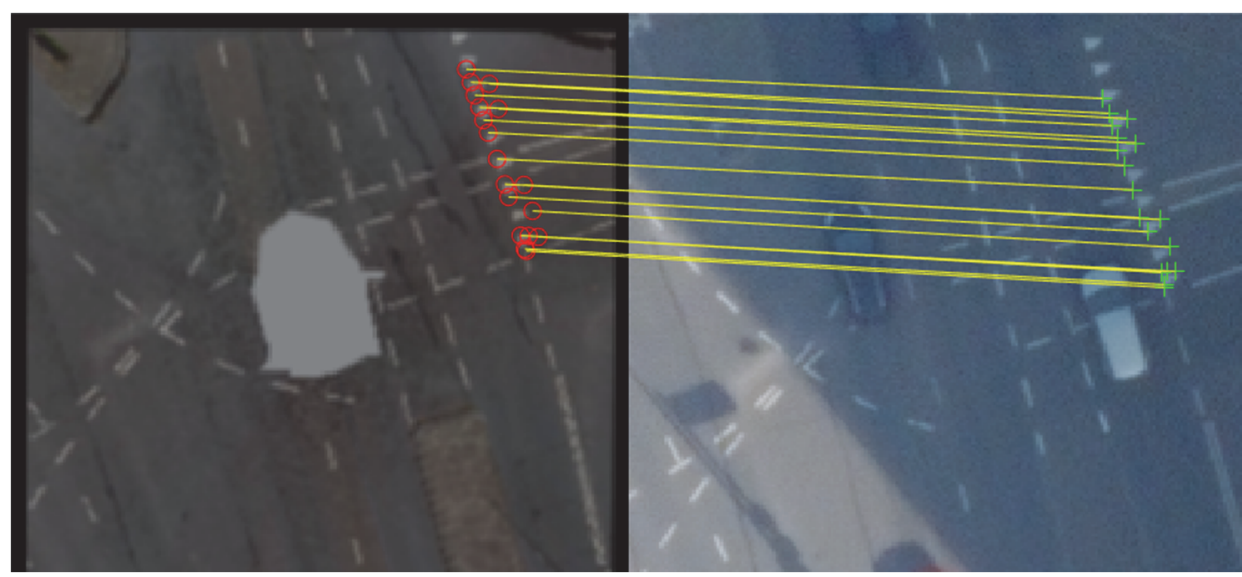

Figure 3.11 Successful registration result between MM and aerial image, although strong illumination differences are present. Please note: Correspondences are initially identified between two MM images, thus other road markings other than the triangular ones in this example were not in the overlapping area of the source MM images.

In some rare cases (one occurrence in all three data sets combined), repeated patterns lead to a wrong computation of the initial transformation (Figure 3.12 on the left) and consequently to a wrong registration result. As at least two aerial images need to be registered with a MM patch, the triangulation procedure will return high variances, and can be thus sorted out.
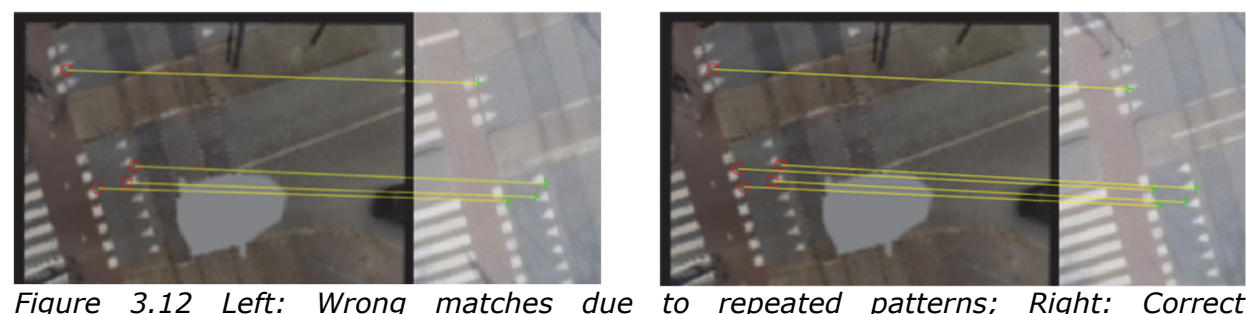

Figure 3.12 Left: Wrong matches due correspondences with a different aerial image.

\subsubsection{Adjustment results}

This section presents adjustment results of the Rotterdam test data set. Table 3-2 shows a comparison of the position updates in the $X, Y$ and $Z$ dimension for the respective test areas. It is evident, that the largest shifts can be 
expected in the horizontal dimension, as these elements have been artificially deteriorated. The Z-component has been left untouched. Hence, updates in the $Z$-dimension may introduce an error due to the unfavourable intersection geometry. The theoretical triangulation accuracy of the aerial data set is about 0.9 metres in height. As recommended earlier, imprecise or wrong correspondences were removed. Nonetheless, a comparison with surveyed GCPs (see Table 3-3, Table 3-4, and Table 3-5) ascertains a larger error in Z in some cases after the adjustment.

Table 3-2 Position updates after adjustment of all three MM test trajectories.

\begin{tabular}{ccccccccccc}
\hline & \multicolumn{3}{c}{ Area 1 } & \multicolumn{3}{c}{ Area 2 } & \multicolumn{3}{c}{ Area 3 } \\
& $X[\mathrm{~m}]$ & $\mathrm{Y}[\mathrm{m}]$ & $\mathrm{Z}[\mathrm{m}]$ & $\mathrm{X}[\mathrm{m}]$ & $\mathrm{Y}[\mathrm{m}]$ & $\mathrm{Z}[\mathrm{m}]$ & $\mathrm{X}[\mathrm{m}]$ & $\mathrm{Y}[\mathrm{m}]$ & $\mathrm{Z}[\mathrm{m}]$ \\
\hline \hline Mean & -0.84 & -0.76 & 0.5 & -0.53 & -0.63 & 0.09 & -0.32 & -0.66 & -0.04 \\
$\begin{array}{c}\text { Standard } \\
\text { deviation }\end{array}$ & 0.14 & 0.32 & 0.03 & 0.438 & 0.516 & 0.125 & 0.376 & 0.359 & 0.062 \\
Max & -0.14 & -0.17 & 0.66 & 0.63 & 0.69 & 0.34 & 0.32 & 0.32 & 0.05 \\
Min & -1.09 & -1.28 & 0.36 & -1.1 & -1.56 & 0.0 & -1.66 & -1.72 & -0.53 \\
\hline
\end{tabular}

In total, 7 GCPs can be observed from the MM images in three test trajectories. GCPs are used as checkpoints, and their respective image observations are manually measured in the MM images, triangulated, and compared with the corresponding GCP's object coordinate (see Figure 3.13 for example). The triangulation has been performed in two different ways. A simple two-view triangulation with the MM images closest to the GCP and a multi-view triangulation (Alsadik, 2016) with all MM images up to a distance of $15 \mathrm{~m}$. 


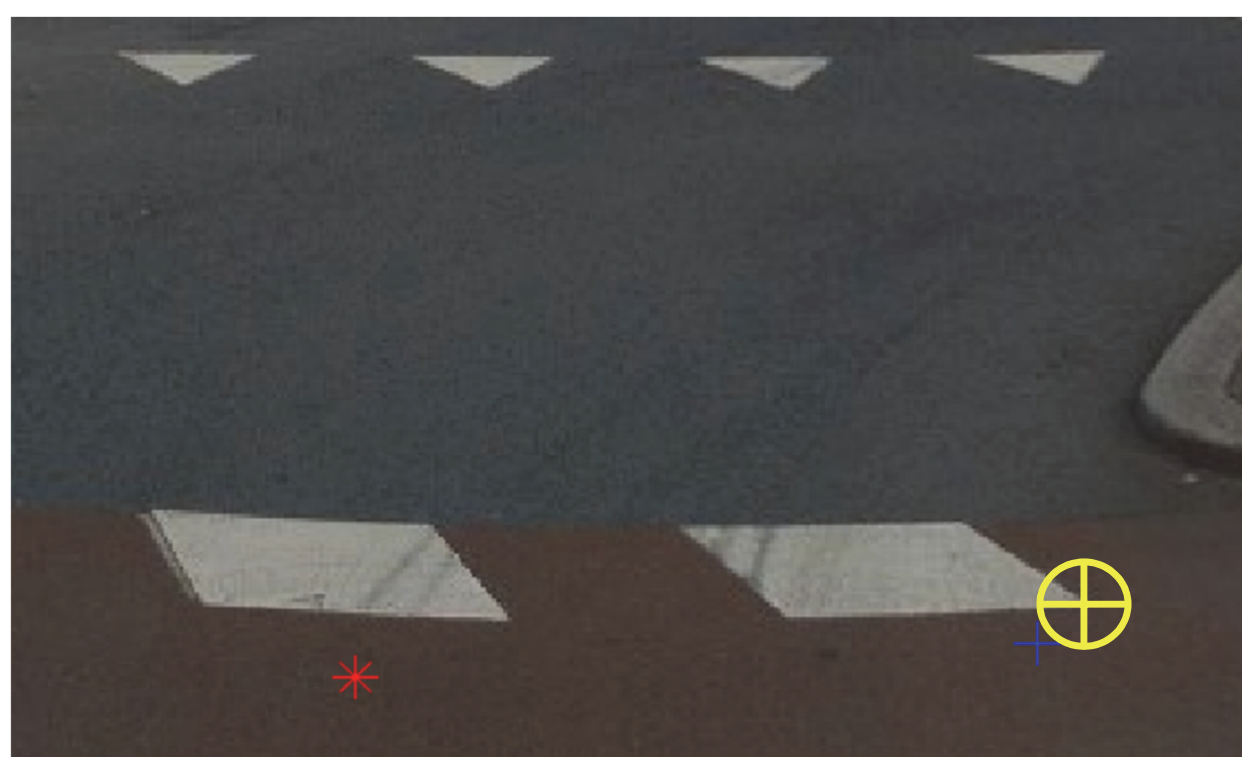

Figure 3.13 GCP measurement example. Red: GCP projected into MM image using original orientation; blue: GCP projected into MM image using updated orientation; Yellow: Actual position of GCP measured manually.

Table 3-3 Error comparison of original and adjusted Area 1 compared to one GCP (please note: no RMSE, as only one GCP available).

Error Multi-View Triangulation Two-View Triangulation

Before After Before After

\begin{tabular}{lcccc}
\hline \hline$X[\mathrm{~m}]$ & 0.574 & 0.143 & 0.568 & 0.15 \\
$Y[\mathrm{~m}]$ & 0.704 & 0.18 & 0.699 & 0.176 \\
$Z[\mathrm{~m}]$ & 0.044 & 0.547 & 0.051 & 0.556 \\
3D $[\mathrm{m}]$ & 0.525 & 0.343 & 0.521 & 0.347
\end{tabular}




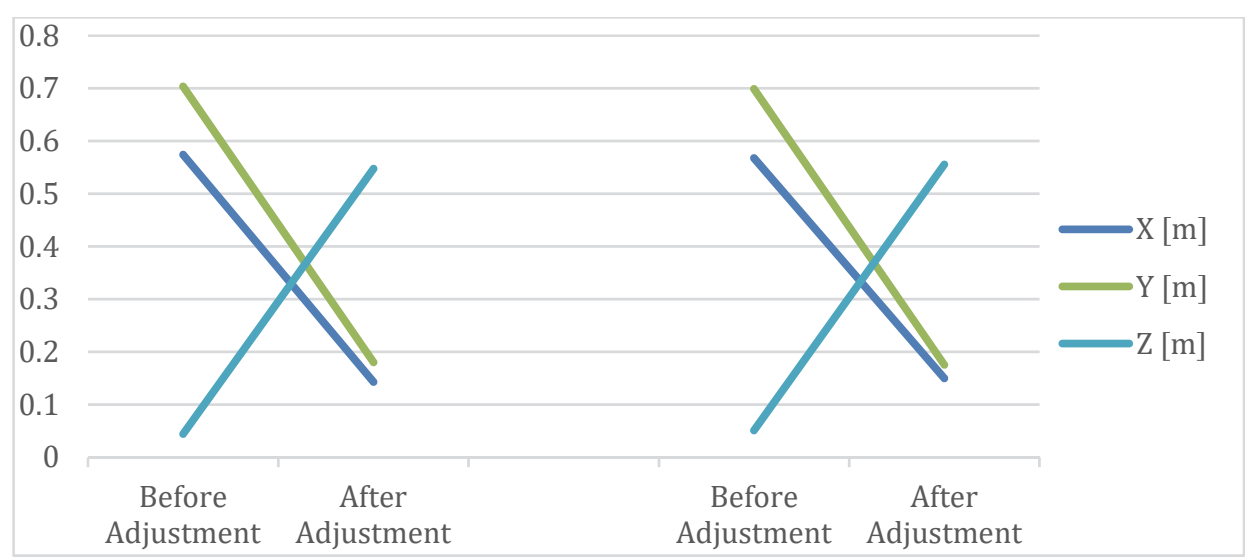

Figure 3.14 Error comparison of original and adjusted Area 1 compared to one GCP; left part: multi-view triangulation; right part: two-view triangulation.

Table 3-4 RMSE comparison of original and adjusted Area 2 compared to three GCPS.

Error Multi-View Triangulation Two-View Triangulation

\begin{tabular}{lcccc} 
& Before & After & Before & After \\
\hline \hline$X[\mathrm{~m}]$ & 1.1 & 0.107 & 1.068 & 0.092 \\
$\mathrm{Y}[\mathrm{m}]$ & 0.946 & 0.134 & 0.984 & 0.096 \\
$\mathrm{Z}[\mathrm{m}]$ & 0.059 & 0.344 & 0.069 & 0.344 \\
3D $[\mathrm{m}]$ & 0.839 & 0.219 & 0.839 & 0.213
\end{tabular}

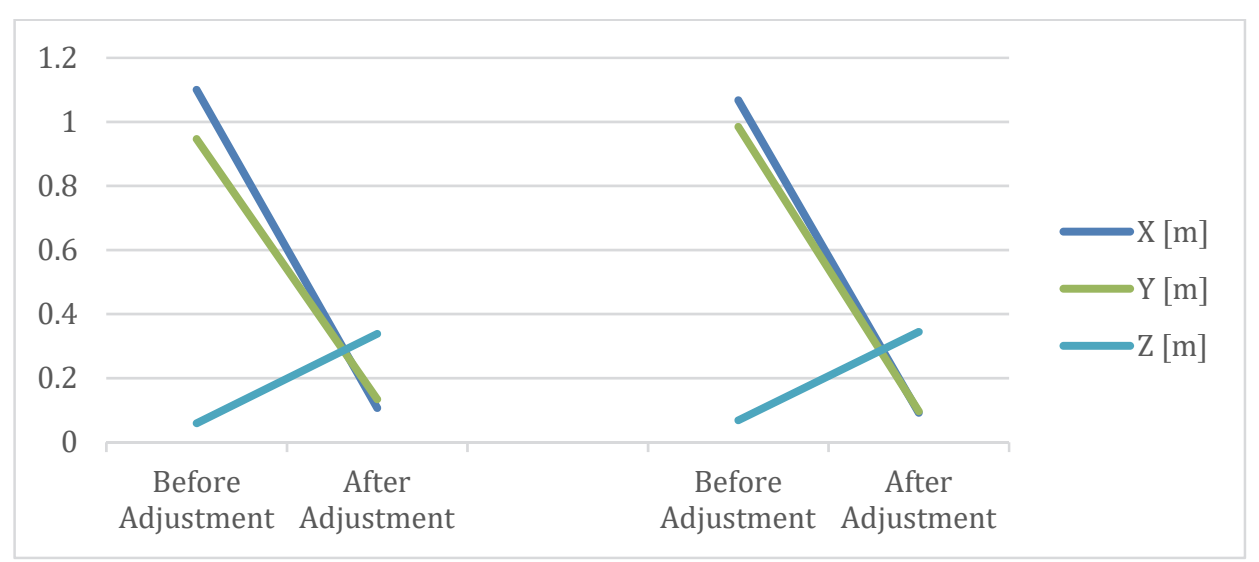

Figure 3.15 RMSE comparison of original and adjusted Area 2 compared to three GCPs; left part: multi-view triangulation; right part: two-view triangulation. 
Table 3-5 RMSE comparison of original and adjusted Area 3 compared to three GCPS.

Error Multi-View Triangulation Two-View Triangulation

Before After Before

\begin{tabular}{lllll}
\hline \hline$X[\mathrm{~m}]$ & 0.822 & 0.082 & 0.736 & 0.397 \\
$\mathrm{Y}[\mathrm{m}]$ & 0.904 & 0.058 & 0.827 & 0.045 \\
$\mathrm{Z}[\mathrm{m}]$ & 0.061 & 0.006 & 0.112 & 0.029 \\
3D [m] & 0.706 & 0.058 & 0.647 & 0.231
\end{tabular}

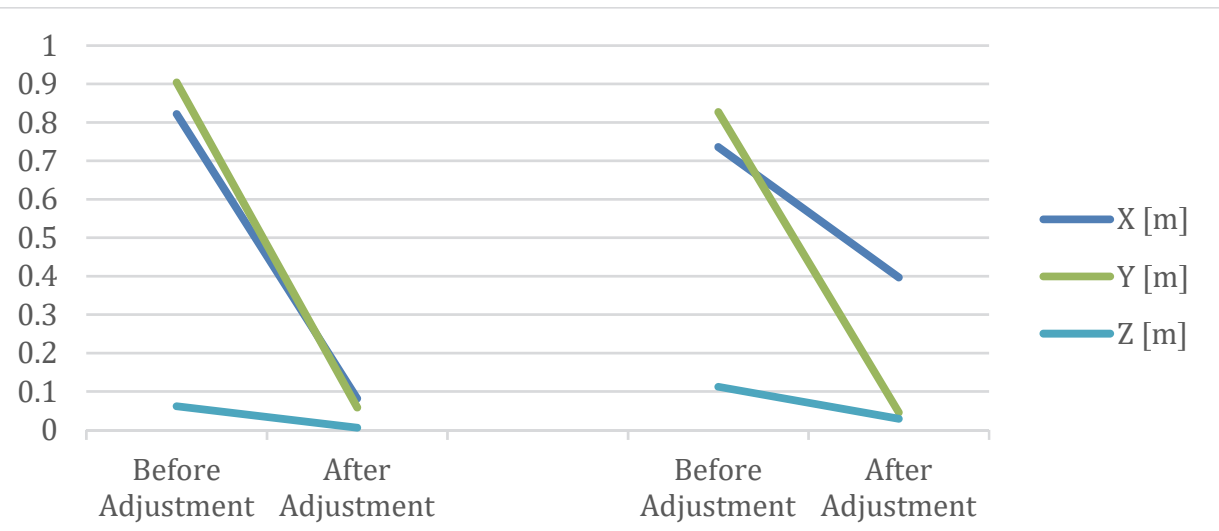

Figure 3.16 RMSE comparison of original and adjusted Area 3 compared to three GCPs; left part: multi-view triangulation; right part: two-view triangulation.

In all three test areas, the accuracy in the horizontal dimension could be highly increased. Location offsets of the platform of about 1 metre could be reduced to be in the low decimetre range. Area 1 features the lowest average accuracy compared to ground truth with an error of $14.3 \mathrm{~cm}$ in $X$ and $18 \mathrm{~cm}$ in $Y$ using multi-image triangulation. Area 2 and 3 ascertain an even better accuracy with Area 3 providing accuracy well below 10 centimetres in all three dimensions. It is noteworthy, however, that the disadvantageous intersection geometry does introduce large errors in Z. Thus, the goal of decimetre accuracy with respect to a three-dimensional adjustment employing an aerial nadir data set with a high acquisition altitude cannot be maintained at all times. On the other hand, a two-dimensional adjustment with the aim to comply with decimetre accuracy is well feasible. It is thus debatable whether the height component should be adjusted as well if the aerial image data had been acquired at a high altitude. 


\subsection{Conclusion}

This chapter outlined a fully automatic registration procedure between MM and aerial nadir data to improve the localisation quality of MM platforms in GNSSdenied areas. An array of techniques has been presented, which is necessary to solve this non-standard registration scenario. With an inlier rate of about $98 \%$ in average, accurate as well as reliable correspondences could be derived. These correspondences enabled an adjustment of MM data, which could demonstrate the feasibility to achieve decimetre-accuracy in GNSS-denied environments.

Currently, the procedure is limited to aerial nadir images and salient features, such as road markings. This may limit the approach to certain areas, although relative constraints, e.g. introduced by $\mathrm{VO}$, can bridge areas without direct correspondences to the reference data set. Moreover, aerial nadir images acquired at a height of about 4500 metres may introduce errors in height, thus a two-dimensional or rather horizontal adjustment is arguably an option in this case.

Future endeavours will focus on the utilisation of oblique images to increase the number of observations as well as to enable a better intersection geometry. Furthermore, the utilisation of deep learned registration approaches may better overcome inter-image differences and thus increase the overall matching performance. Especially within the context of oblique images, not only ground features will become available (but e.g. complex façade structures), and thus learning approaches can be considered a viable alternative to template matching. 


\section{Chapter 4-Co-registration of panoramic mobile mapping images and oblique aerial images $^{19}$}

\footnotetext{
19 This chapter is based on:

Fanta-Jende, P., Nex, F., Vosselman, G. \& Gerke, M. (2016) 'Co-registration of panoramic mobile mapping images and oblique aerial images', The Photogrammetric Record, Vol.
} 35, https://doi.org/10.1111/phor.12276 


\subsection{Abstract}

Mobile mapping relies on satellite-based positioning, which suffers from line-ofsight and multipath issues. As an alternative, this chapter presents a fully automatic approach for the co-registration of mobile mapping and oblique aerial images to introduce highly accurate and reliable ground control for mobile mapping data adjustment. An oblique view of a scene introduces similarities as well as challenges regarding co-registration with mobile mapping images, which is supported by mutual planes in both datasets. Façade planes from a sparse point cloud are used as projection surfaces for the mobile mapping and aerial datasets, overcoming large perspective differences between them to simplify the registration. The performance of the procedure indicates an inlier rate of around $80 \%$. 


\subsection{Introduction}

Mobile mapping has become an established technique to acquire large, highresolution datasets, predominantly in urban areas, at relatively low cost. As MM can be regarded as a complementary acquisition technique to aerial photogrammetry, it enables intriguing applications from a terrestrial point of view in computer vision, autonomous driving and robotics. With high-precision positioning equipment for absolute and relative localisation, MM accuracy is comparable to survey-grade technologies.

Since the absolute position of these platforms is provided by Global Navigation Satellite Systems (GNSS), such as GPS or GLONASS, MM campaigns are directly affected by typical implications of these systems. Multipath and lineof-sight deficiencies impede fixing an accurate absolute position by GNSS, particularly in urban areas where, for instance, high-rise buildings may obstruct the direct line of sight to GNSS satellites. Consequently, this leads to wrong positional estimations of the MM platform and thus an unknown error in the data product. In order to correct or compensate for this issue, various techniques have been used. For instance, surveyed ground control points (GCPs) (Cavegn et al., 2016) or digital maps (Levinson and Thrun, 2007) have been introduced into the adjustment of MM data products. In any case, external references are required to either verify, or increase the accuracy of, the dataset.

The method presented in this chapter utilises oblique aerial imagery as a tertiary dataset. This can be regarded as a logical continuation of previous efforts, where aerial nadir images were used within the same context (Jende et al., 2018a). Exclusively utilising aerial nadir images is limited to groundbased correspondences only, which has certain disadvantages with respect to the availability of correspondences and an adverse geometry within the adjustment solution. Utilising aerial oblique images, however, enables finding correspondences in narrow alleyways on façades or other vertical objects, and hence increases the geometrical stability within an adjustment due to a higher number of correspondences.

Although, a registration of MM perspective and oblique aerial images poses similar challenges, especially with respect to overcoming perspective differences, the approach discussed in this chapter is different from the vertical aerial registration approach in many instances. For example, a registration with vertical aerial images is based on the assumption of a flat ground plane, which allows for a remapping of the MM panoramic image. In the present case of oblique aerial images, however, mutual planes between the image datasets need to be identified first. 
The structure of this chapter is as follows. First, an overview of related and previous work is given. The next section discusses all relevant strategies and methods to register MM perspective and oblique aerial images. The following section comprises an extensive set of experiments. Lastly, special cases and possible advancements are discussed.

\subsection{Related Work}

In this work, MM data are registered with aerial image data to introduce independent ground control for data adjustment. Two areas of research overlap in such an approach, namely wide-baseline/non-standard geometry image registration as well as localisation and positioning. Image registration is a research topic that is widely covered in the literature. Although the field is moving towards machine learning and is able to cope with wide-baseline problems (Lin et al., 2015; Melekhov et al., 2017; Shan et al., 2014; Yicong Tian, 2017; Yixiang Tian, 2014; Zagoruyko and Komodakis, 2015), these approaches are still very experimental and cannot provide the reliability and registration accuracy required for the task at hand. Alternatively, authors rely on synthesised views to homogenise the datasets to be registered (Morel and Yu, 2009; Roth et al., 2017).

Similarly, in the authors' previous work, MM panoramic images were reprojected onto an artificial ground plane to achieve a higher resemblance to a vertical aerial (nadir) dataset (Jende et al., 2018a). The image registration problem can be constrained using guided matching strategies, as the absolute orientation (and thus the positioning error) is within an expected margin of error. Consequently, the challenge is identifying accurate and salient features in both datasets rather than sensor-specific correspondences. This translates the problem to finding salient, repeatable corner features by feature detection algorithms, such as the adaptive and generic accelerated segment test (AGAST) proposed by Mair et al. (2010b). Since these corners are used to register MM images with each other to obtain a sparse point cloud, as well as serving as the basis for a registration with oblique aerial images, repeatable recognition of distinctive façade elements, such as window frames or gutters, is the most important property.

Regarding the localisation problem in GNSS-denied areas, available approaches can be coarsely separated in two different categories:

1. methods which focus on the correction of the data product itself (as in the current approach); and

2. methods which aim for the correction of the platform's trajectory for the purpose of correct localisation. 
Whereas, the latter category is mainly suitable for real-time applications, correcting the data product in (1) allows for post-processing. This difference has an influence on the methodology. Although a method could be designed to correct data directly during acquisition, post-processing enables the factoring out of GNSS effects on the positioning to a greater extent.

Real-time capable applications, such as simultaneous localisation and mapping (SLAM) or visual odometry, allow for the introduction of external references by map-aiding (Gruyer et al., 2014; Gu et al., 2016; Roh et al., 2016; Schindler, 2013) or shadow matching (Groves et al., 2013; Irish et al., 2015; Strode and Groves, 2016); the latter classifies GNSS signals with respect to their angle of incidence. It should be noted that there are a multitude of approaches which do not utilise external data, but rather optimise the trajectory using onboard sensor systems (Balazadegan Sarvrood et al., 2016; Carlone and Karaman, 2017; Zhang and Singh, 2015). These approaches, however, cannot achieve the desired accuracy.

On the other hand, post-processing approaches traditionally rely on GCPs, which are introduced into the adjustment (Cavegn et al., 2016; Han and Lo, 2016; Hofmann and Brenner, 2016; Molina et al., 2017). This proved to be accurate but is labour intensive and thus costly. Javanmardi et al. (2017) and Ji et al. (2015), for instance, also utilised aerial images to introduce an external reference into the solution. These approaches only utilise vertical aerial images, and thus have similar limitations to the current authors' previous approach as far as the availability of potential correspondences is concerned. Merging oblique aerial images, as well as terrestrial images, for creating complete representations of a scene has been pursued by Wu et al. (2018). Although this is a different task with the image data already co-registered with respect to their orientation, a method similar to the one used in the Wu paper is used, as those authors relied on plane priors extracted from 3D meshes. Furthermore, the necessary point cloud was derived from an aerial dataset. In contrast to this, the approach in this chapter relies on a point cloud derived from mobile mapping images, which is cheaper to compute and potentially yields a more accurate representation of the scene and thus a more reliable registration result. Among other major differences, feature matching between rectified images is utilised rather than template matching using image patches.

\subsection{Methodology}

This section outlines the registration algorithm in detail (see Figure 4.1 for an overview). It is designed to work in a fully automatic fashion, where MM images are processed sequentially with respect to their location in the trajectory. There are four main steps: 
1. MM images are registered with each other to yield a sparse point cloud. This step is also a requirement for the adjustment procedure, as it allows for propagating a position update across multiple MM recording locations, and updating of positions of recording locations without direct correspondences with the aerial dataset.

2. The sparse point cloud is used for identifying planar surfaces along the trajectory, particularly building façades. If predefined criteria for a plane are met, such as visibility from both datasets and orientation, the respective oblique aerial and $\mathrm{MM}$ images are projected onto patches coinciding with that plane.

3. Patch-based registration is conducted, determining the translation between both patch centres.

4. A consensus based on the median translation between the MM and oblique aerial patches is computed to remove outliers.

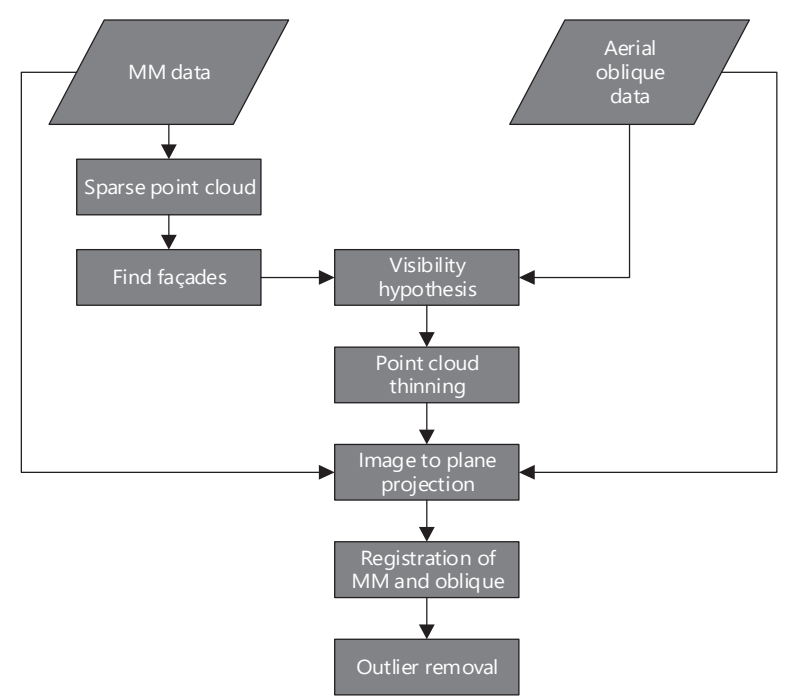

Figure 4.1 Mobile mapping to aerial oblique image registration pipeline.

\subsubsection{Sparse Point Cloud from Mobile Mapping Images}

A prerequisite to the MM-to-oblique-aerial registration procedure, as well as the adjustment of the MM dataset, is the generation of a sparse point cloud. The procedure is designed to identify correspondences, particularly on building façades, which are assumed to be approximately parallel to the trajectory of the MM platform. A subsequent plane fitting identifies those points of the 
sparse point cloud, which coincide with a putative façade. Moreover, inlying points are used for patch creation, which is required for the actual registration between the MM and oblique datasets. MM panoramic images are encoded in a spherical equirectangular projection. This projection entails strong distortions in comparison to common perspective images (see Figure 4.2).

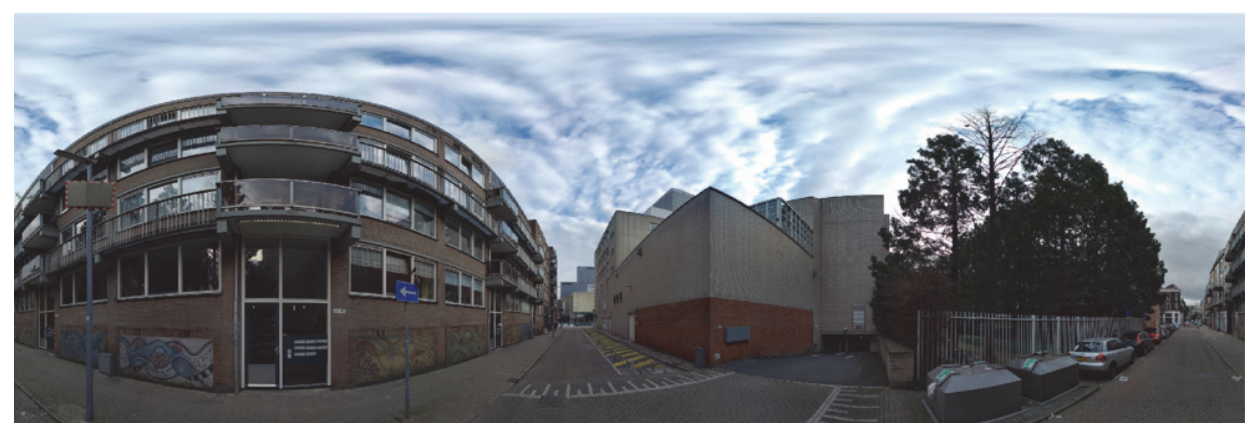

Figure 4.2 Equirectangular panoramic image.

To enable reliable feature matching across multiple panoramic images while ensuring a similar coverage of the scene, panoramic images are transformed to perspective images with specific yaw deviations from the driving direction. For each iteration, three perspective images for each side (left/right of the trajectory) are derived from three panoramic images. The principle of differing yaw deviations is depicted in Figure 4.3 (a). For the left-hand side of the trajectory, for instance, the first perspective image in the trajectory is created with a yaw deviation of $300^{\circ}$, the second one with $270^{\circ}$ and the third one with a yaw deviation of $240^{\circ}$. Employing that configuration, the orientation of the perspective images coincides with the position of recordings in the trajectory in such a way that the same scene in object space can be depicted from different angles (Figure 4.3 (b)). In order to capture building façades, perspective images are generated with a fixed pitch angle of $30^{\circ}$ above the horizon (Figure 4.4).

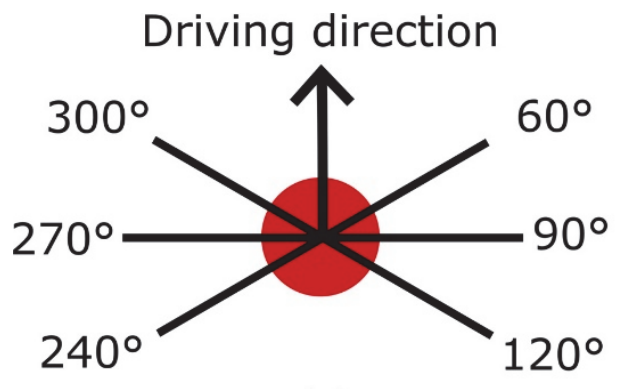

(a)

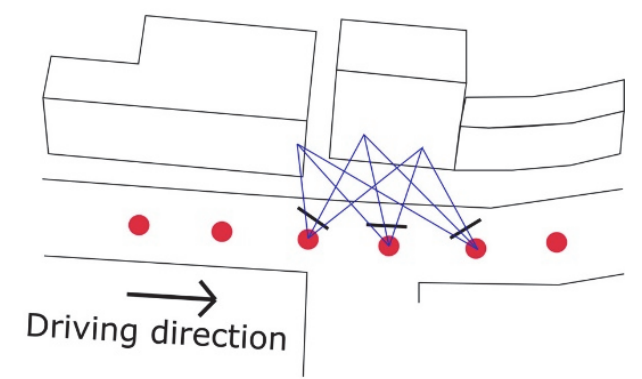

(b) 
Figure 4.3 (a) Yaw deviations for perspective image creation. (b) Principle of sparse point cloud generation based on image triplets.

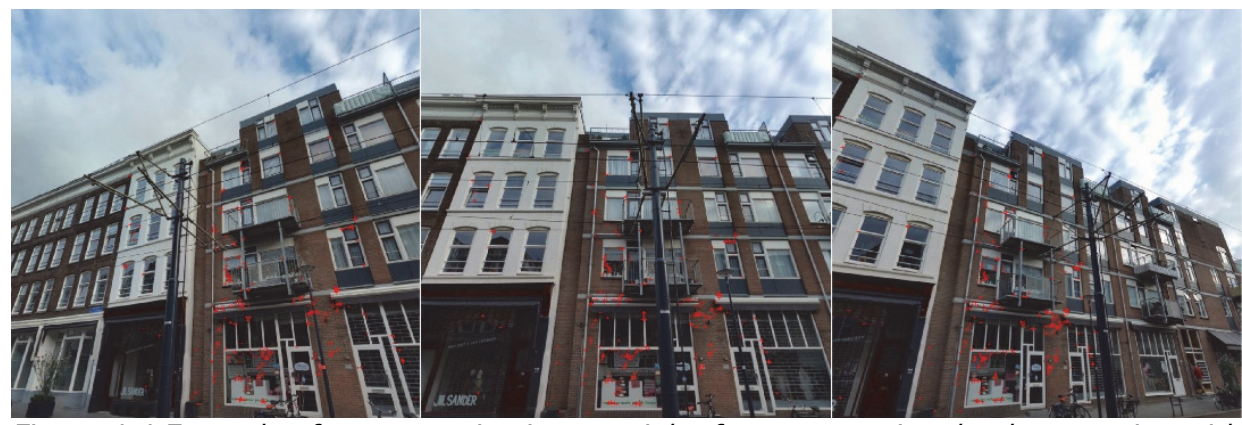

Figure 4.4 Example of a perspective image triplet for sparse point cloud generation with respective correspondences. From left to right, images 1, 2 and 3.

Feature matching is conducted using a combination of the AGAST detector (Mair et al., 2010a) and the DAISY descriptor (Tola et al., 2010). As a corner detector, AGAST proved to be useful for façade matching since it identifies features where image gradients intersect, rather than distinctive image areas by blob detection. Because these correspondences are re-used in a later step for patch creation and for the registration with oblique images, these image patches are, for instance, derived at and around window frames, which potentially returns more salient features than patches from arbitrary image areas.

The registration procedure is based on perspective image triplets, such as images 1,2 and 3 in Figure 4.4. Feature matching is conducted between images 1 and 2, and between images 2 and 3. The feature set of image 2 is used to link images 1 and 3 as well. In contrast to panoramic images, these artificial images employ a pinhole camera model. In combination with known extrinsic (exterior) elements and rotations, it allows for the derivation of projection matrices and hence a trifocal tensor (three-photo relative orientation). Employing the trifocal point-transfer constraint enables reliable outlier removal. First, the focal length (principal distance) $f$ for artificial perspective views needs to be determined, starting from an ideal opening angle (field of view):

$$
f=\tan \left(90^{\circ}-\frac{v f o v}{2}\right) * w / 2
$$

where $v f o v$ is the vertical field of view and $w$ is the image width.

The camera matrix $\mathbf{K}$ only has to be determined once, as the intrinsic (interior orientation) parameters of artificial views do not change: 


$$
\mathbf{K}=\left[\begin{array}{ccc}
f & 0 & w / 2 \\
0 & f & w / 2 \\
0 & 0 & 1
\end{array}\right]
$$

Subsequently, respective rotations $\mathbf{R}_{\boldsymbol{i}}$ for every view of the triplet need to be computed. The rotations into the camera frame are already included. (Note the sequence of the rotations.)

$$
\begin{aligned}
& \mathbf{R}_{\mathbf{i}} \\
& =\left(\begin{array}{ccc}
1 & 0 & 0 \\
0 & \cos \left(90^{\circ}\right) & -\sin \left(90^{\circ}\right) \\
0 & \sin \left(90^{\circ}\right) & \cos \left(90^{\circ}\right)
\end{array}\right)\left(\begin{array}{ccc}
\cos (\omega) & 0 & \sin (\omega) \\
0 & 1 & 0 \\
-\sin (\omega) & 0 & \cos (\omega)
\end{array}\right)\left(\begin{array}{ccc}
1 & 0 & 0 \\
0 & \cos (-\varphi) & -\sin (-\varphi) \\
0 & \sin (-\varphi) & \cos (-\varphi)
\end{array}\right)\left(\begin{array}{ccc}
\cos (\kappa) & -\sin (\kappa) & 0 \\
\sin (\kappa) & \cos (\kappa) & 0 \\
0 & 0 & 1
\end{array}\right)
\end{aligned}
$$

where $\varphi$ is the pitch angle for camera (fixed to $30^{\circ}$ to capture façades), $k$ is the yaw rotation including defined yaw deviations (see Fig. 3(a)), and $\omega$ is the roll angle (fixed to $0^{\circ}$ ).

Now, the projection matrices $\mathbf{M}_{i}$ can be determined using:

$$
\mathbf{M}_{i}=\mathbf{K}\left[\mathbf{R}_{i} \mid \mathbf{t}_{c}\right]
$$

where $\mathbf{t}_{c}$ are the world coordinates (object coordinates) of the respective panoramic image. After the projection matrices for each perspective view of the two triplets have been obtained, two trifocal tensors can be computed, one for each side of the trajectory. The computation and further details can be found in Hartley and Zisserman (2004). In order to remove outliers, the trifocal point transfer (equation (8) in Hartley (1997)) is used. By selecting a putative correspondence in two views, the trifocal tensor is used to determine the correspondence's location in the third image. After comparing the actual image coordinate from feature matching to the computed one, a wrong correspondence can be removed unless the configuration is degenerate, thus ensuring points lie on the same epipolar line.

Subsequent to feature matching and outlier removal, the remaining correspondences are translated into the original equirectangular projection for the triangulation (intersection) of object points. Although intersection would be possible using the perspective geometry, for subsequent steps the equirectangular image coordinates are required.

\subsubsection{Plane Fitting to Identify Façades}

Plane fitting is used to determine whether object points coincide with a building façade. Other objects, such as trees, cars and traffic lights, are unfit for registration and can be thus rejected. In this approach, only façades that are parallel to the trajectory are considered (thus building frontages facing the street along which the MM vehicle is travelling). To prevent instabilities of the process at the expense of flexibility, a collinearity criterion with respect to the recording locations is employed. Subsequently, a vector perpendicular to the 
driving direction is defined as a reference vector for plane fitting. Thus, an angular threshold determines the maximum offset between the reference vector and the plane's normal vector. In the "Experiments" section, it will become evident that this threshold has a great impact on the number of inlying object points, and hence possible correspondences.

As façades may have some projections, such as balconies or oriels, a plane can only approximate its geometry. To account for this, a maximum deviation of $0.5 \mathrm{~m}$ for object points is defined. Plane fitting is based on maximum likelihood estimation sample consensus (MLESAC; Torr and Zisserman (2000)). After a plane is identified, the number of object points between the plane and the recording location is used to determine whether the plane is occluded. Although, a plane may have been identified properly, occluded planes may impede the remapping of image information on a façade later, and consequently complicate the registration task (see Figure 4.5).

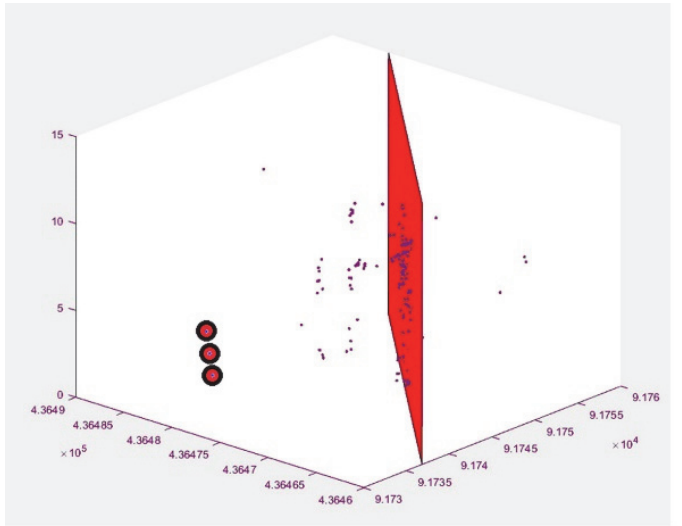

(a)

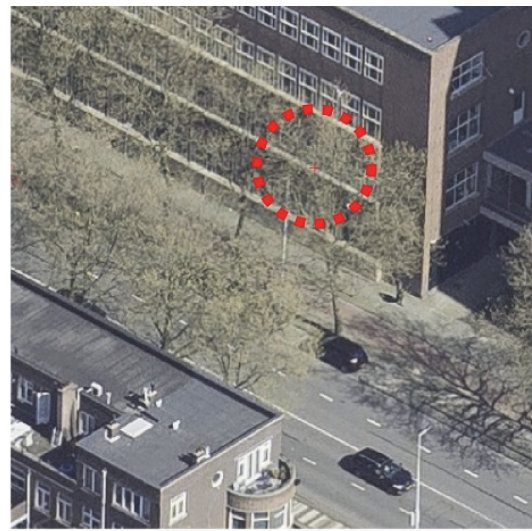

(b)

Figure 4.5 A façade occluded by vegetation. (a) Point cloud (purple points), recording locations (red circles edged in black), fitted plane (red). (b) Oblique aerial image of the scene, where the red circle indicates the plane centre in the scene.

\subsubsection{Visibility Hypothesis}

Visibility of a façade is an important prerequisite for the registration between MM and oblique aerial images. Especially in urban areas, high-rise buildings may lead to occlusions with respect to oblique aerial images. Although, no surface or building model is used in the procedure, a series of methods can be employed to determine if a set of inlying points is visible from respective oblique images. Three criteria have to be fulfilled:

1. Two angles, $\varphi$ and $\theta$, are computed to ascertain the orientation of a façade with respect to an individual oblique aerial camera (Figure 4.6). Therefore, inlying points are averaged to determine their centre (centroid) coordinate (pavg) to obtain the perpendicular foot $\left(\mathbf{c}_{\mathbf{f}}\right)$ of the oblique camera ( $\mathbf{c o b l}_{\mathbf{l}}$ ). 
Subsequently, $\theta$ can be computed as the angle between the plane's normal vector $\mathbf{n}$ and the vector $\overrightarrow{\mathbf{c}_{\mathrm{f}} \mathbf{p}_{\text {avg }}}$ as well as $\varphi$ between $\overrightarrow{\mathbf{c}_{f} \mathbf{p}_{\text {avg }}}$ and $\overrightarrow{\mathbf{c}_{\text {obl }} \mathbf{p}_{\text {avg }}}$.

$$
\begin{gathered}
\theta=\arctan 2\left(\left\|\overrightarrow{\mathbf{c}_{o b l} \mathbf{p}_{a v g}} \times \overrightarrow{\mathbf{c}_{f} \mathbf{p}_{\text {avg }}}\right\|, \overrightarrow{\mathbf{c}_{\text {obl }} \mathbf{p}_{\text {avg }}} \cdot \overrightarrow{\mathbf{c}_{f} \mathbf{p}_{\text {avg }}}\right) \\
\varphi=\arctan 2\left(\left\|\overrightarrow{\mathbf{c}_{f} \mathbf{p}_{\text {avg }}} \times \overrightarrow{\mathbf{n}}\right\|, \overrightarrow{\mathbf{c}_{f} \mathbf{p}_{\text {avg }}} \cdot \overrightarrow{\mathbf{n}}\right)
\end{gathered}
$$

A threshold of $70^{\circ}$ for both angles has been set. For instance, if $\theta$ were larger than $70^{\circ}$, the plane may be still visible, but its representation in the oblique image would be too low for patch creation.

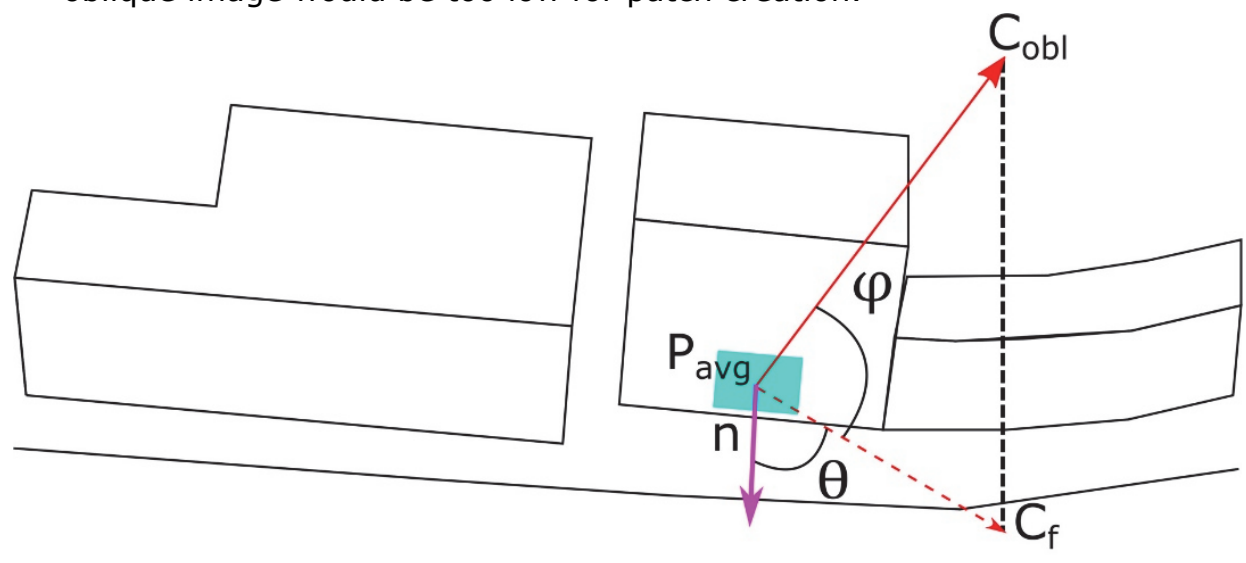

Figure 4.6 Principle of façade visibility for oblique aerial images.

2. The theoretical resolution $(t)$ at the centroid (pavg) with respect to an individual oblique aerial image, based on its focal length, is determined. A threshold of $8 \mathrm{~cm}$ is defined as minimum admissible spacing between the oblique image's pixels, projected onto the wall plane. If the resolution is too low and may impede a solution, the registration is determined later in the patch-creation step.

3. The centroid Pavg is back-projected into the candidate oblique aerial images, to check if the point lies within the aerial image's extent. Although the created image patch will be incomplete in cases where its border does not lie within an oblique image's extent, a registration may be still successful as the registration is conducted with respect to the patch's centre.

These methods can reduce the number of points considered for registration considerably. However, due to the lack of a 3D model, ray tracing or similar 
techniques are not possible, and thus false positives may occur. In most of these cases, a registration will fail. To analyse the actual registration performance properly in the Experiments section, however, these false positives will be labelled 'not matchable' (see Figure 4.7 for an example). In a subsequent adjustment these observations would, most likely, be identified as outliers, given the model is strong enough. A detailed evaluation of this problem is subject to future work.
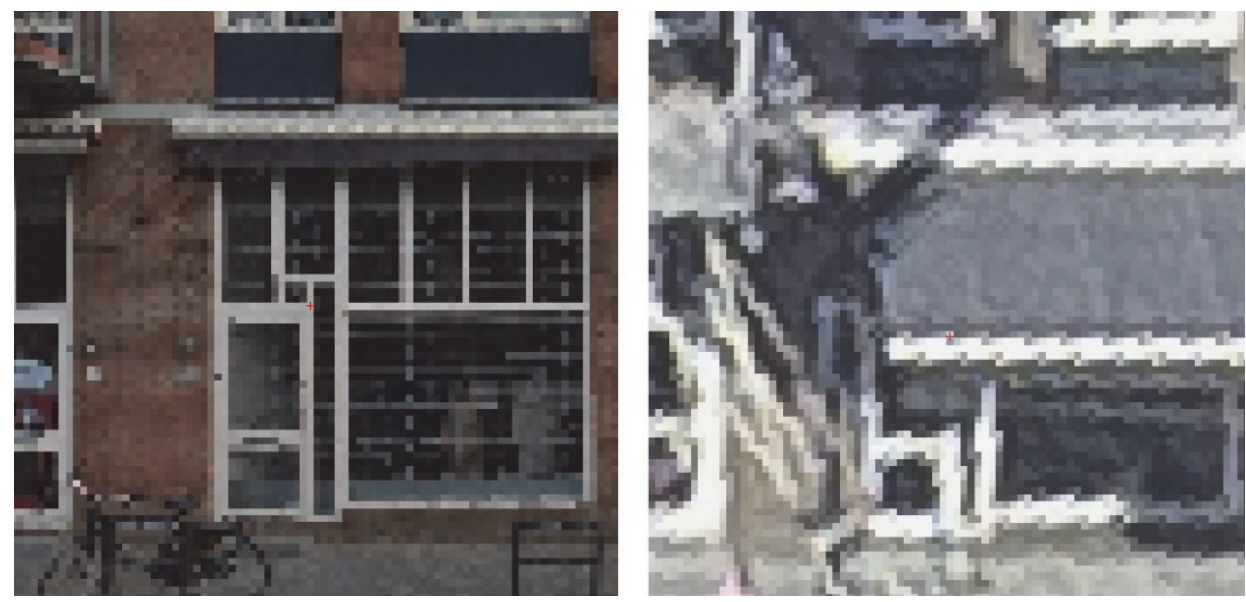

Figure 4.7 An example of a façade which is not matchable, since the awning above the windows in the left image is occluding most of the patch.

\subsubsection{Point Cloud Thinning}

The algorithm to derive a sparse point cloud is based on feature matching techniques. Non-maximal suppression is used during feature detection, which prevents detection of adjacent corners. Since this is a local operation, corners can still be detected in close proximity to one another. Consequently, parts of the point cloud may be dense. This may lead to the generation of almost identical image patches, which only differ a little in translation. This affects both MM and oblique aerial patches to the same extent. On the other hand, more object points lying on a potential façade lead to better and more reliable plane fitting. To this end, thinning of the point cloud is only applied after a plane has been identified. The input of the thinning process is all object points which coincide with a plane (inlying points). A non-uniform box grid filter is used to reduce the number of points (Pomerleau et al., 2013). A maximum number of points per box is defined, from which one point is randomly retained. This process is adaptive, as points with high proximity, rather than isolated points, will be removed. This is a useful property, since the general distribution of the original inlying points can be maintained while cluttered points are removed. 


\subsubsection{Image to Plane Projection}

Panoramic MM and oblique aerial images depict a scene from different perspectives. In order to simplify the registration problem, mutual planes that are potentially visible from both datasets have been identified. In order to register both datasets with each other, image patches are created. As mentioned earlier, a plane does not necessarily comply with the real geometry of a façade. As already noted, object points with a maximum distance from the plane of up to 0.5 metres are still labelled as inlying to compensate for deviations from a true plane in real-world façades. In order to account for these offsets during registration, image patches are created with an object point as its centre (centroid). To discretise an image patch in 3D space, a local coordinate system constituting the respective patch is defined. The corresponding object point is defined as its origin. The coordinate system is derived as follows. All inlying points (pabs) are normalised with respect to their centroid $\mathbf{p a v g}$ to obtain a covariance matrix $\mathbf{C}$ :

$$
\mathbf{C}=\sum_{i=1}^{m}\left(\mathbf{p}_{i}-\mathbf{p}_{a v g}\right) \cdot\left(\mathbf{p}_{i}-\mathbf{p}_{a v g}\right)^{\mathrm{T}}, \quad \mathbf{p}_{i} \in \mathbf{p}_{a b s}
$$

where $m$ is the entire set of inlying points $\mathbf{P a b s}_{\text {abs }}$ Since a covariance matrix is symmetric, its eigenvectors are orthogonal to each other and can be used to define a coordinate system in conjunction with the plane's normal vector (Figure $4.8(a)$ ). In order to discretise a grid the sampling rate, as well as the extent of the image patch, are used. The sampling rate corresponds to the theoretical resolution $t$ of centroid $\mathbf{P a v g}_{\text {avg }}$ with regard to an individual oblique image. The extent of the image patch is, by default, defined as 8 by 8 metres. In the case of hierarchical matching (discussed in the next section), the extent is reduced to 6 by 6 metres in the second iteration. 


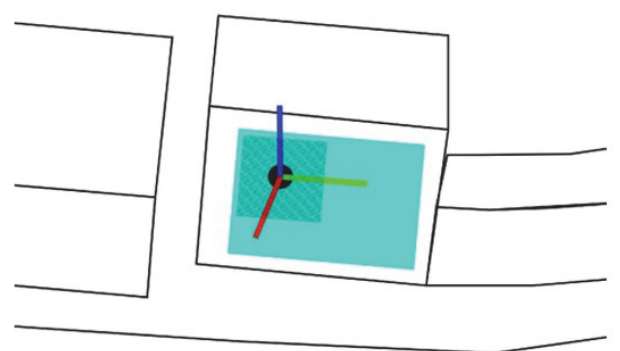

(a)

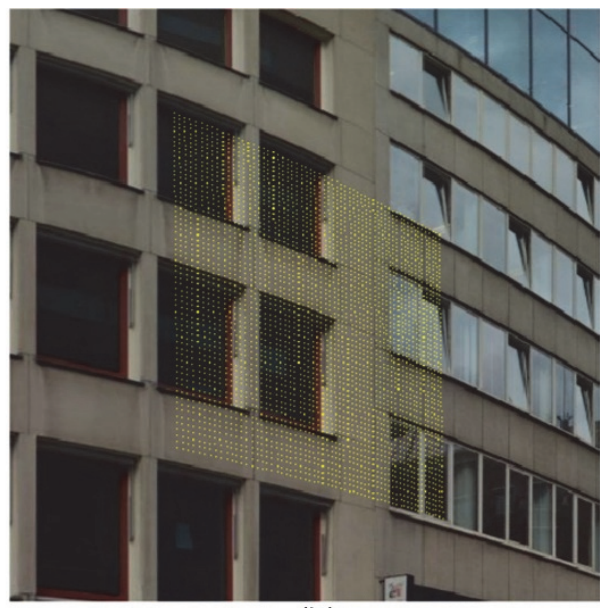

(b)

Figure 4.8 (a) Schematics of patch creation. The eigenvectors (blue and green) and the plane's normal vector (red) constitute the local coordinate system, where the object point (black) on the plane (cyan) is defined as the centre of the discretised grid (grey). (b) Example of a grid (central area in yellow) projected into a panoramic image.

Subsequently, the resulting discretised grid in object space is filled with $\mathrm{red} / \mathrm{green} / \mathrm{blue}(\mathrm{RGB})$ values from both image sources via a back-projection mechanism. This results in two image patches per inlying object point (Figure 4.9). Although the theoretical resolution has been computed earlier, it does not necessarily correspond to the real resolution. This would only be the case if the grid was parallel to the oblique aerial image's image plane. In the scenario in this research, a grid is usually coplanar with the building façades. In order to obtain the real resolution of a grid in a certain oblique image, an intuitive solution would be a projection of the grid into the image itself. However, a simpler solution in this context is to relate the number of assigned individual oblique image's RGB values to the pixels in the grid. In other words, if the theoretical resolution corresponded to the real resolution while the grid is parallel to an oblique image's image plane, the ratio between assigned RGB values and the number of pixels in the grid would be one, since a one-to-one assignment would be possible. In a real-world scenario, however, a single RGB value is retrieved, more often by the back-projection mechanism reducing the ratio and thus the real resolution of the image patch. A threshold for the ratio of 0.6 prevents a low real resolution and hence blur in the oblique image patch, which may impede the registration (Figure 4.10). 

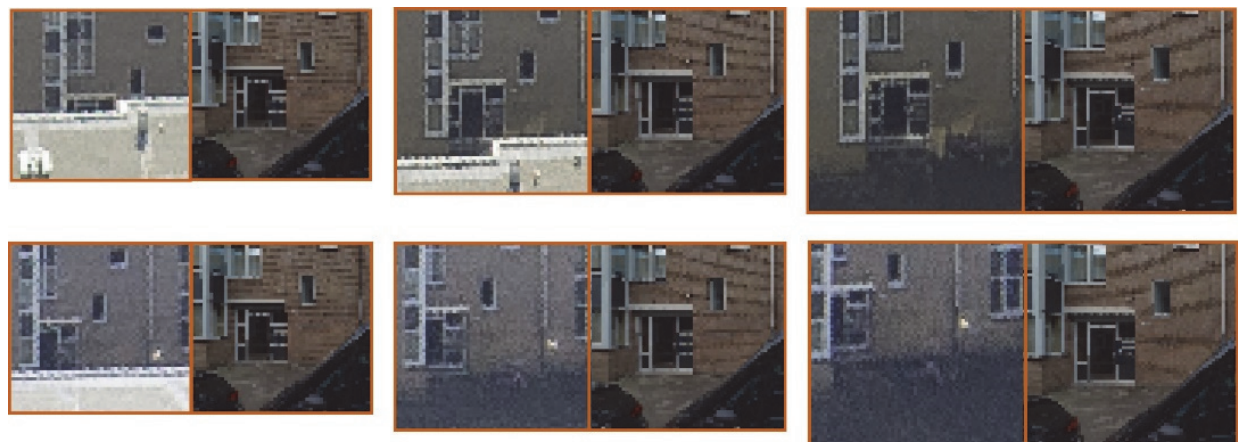

Figure 4.9 Six pairs of oblique aerial (left side of every pair) and panoramic (right side) image patches of the same grid. The oblique aerial image patches are retrieved from different oblique aerial images, hence the partially occluded part. Note the different resolutions of the oblique aerial image; however, the panoramic image in a pair shares the same sampling.
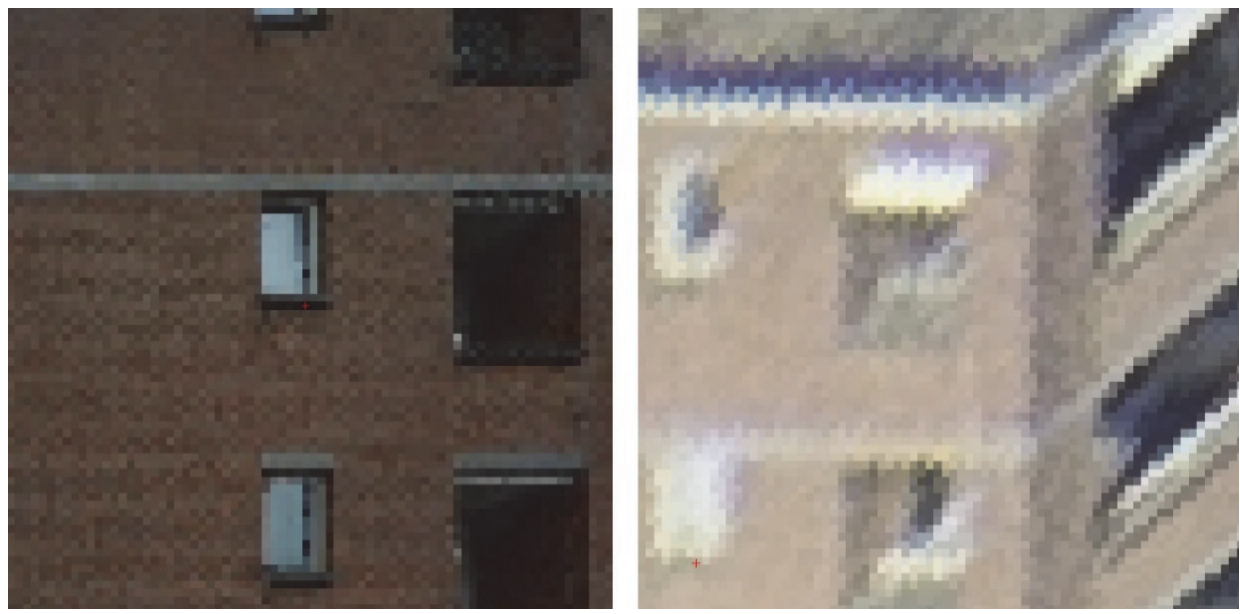

Figure 4.10 MM image patch (a) with corresponding oblique aerial patch (b). Due to the low resolution of the oblique aerial patch, this pair cannot be used for registration.

\subsubsection{Registration}

Image patches simplify the registration scenario considerably, as variance between both datasets can be overcome. For instance, the image patches share a similar (but not exactly the same) scale, perspective and rotation. Hence, the registration problem is simplified to finding the correct translation between a MM and an oblique aerial image patch. As the registration is based directly on image patches, the translation is interpreted as the shift between the patch centres, which are the respective image observations of the object point previously labelled as an inlier in the plane fitting procedure.

Although the image patches share a number of similarities, a patch-based registration requires context to find the correct transformation. To this end, an 
optional hierarchical registration approach was implemented which firstly finds an initial, but coarse, transformation between larger patches with a lower resolution. In a second iteration, the initial transformation is used to constrain the registration for a more accurate result. Besides minor computational speed improvements, the main benefit of hierarchical matching is a higher inlier rate. More details comparing hierarchical and non-hierarchical matching are analysed in the next section. In both cases, template matching techniques are employed. A coarse registration is conducted using normalised cross correlation, whereas a fine registration is performed using mutual information as a similarity criterion (Mattes et al., 2001). For mutual information, a oneplus-one evolutionary algorithm is used to find the best parametrisation (Droste et al., 2002). This algorithm modifies its set of parameters every iteration until it converges to the best registration result.

In general, utilising mutual information proved to be a powerful method for the scenario highlighted in this chapter, as it can overcome illumination details while ignoring differing image content. Even though outliers may be still present, it performed better for fine registration than, for example, normalised cross correlation, which is more sensitive to noise (see experiment 8/9 in the next section).

\subsubsection{Outlier Removal}

Since a correspondence between a pair of image patches can be regarded as either the translation between image observations or a translation in object space, two outlier removal techniques have been devised: a 2D and a 3D case. A set of correspondences is defined as all correspondences between one panoramic image and one oblique aerial image. The difference between the two outlier removal techniques is their dimension. In the 2D case, image observations (row/column) are fed into the procedure. In the 3D case, a MM image correspondence coincides with an inlying object point (pabs), whereas an oblique aerial image correspondence is converted into 3D by relating it to the grid in object space. In both cases, a consensus based on the median translation is used. If a correspondence deviates from the median value by a certain threshold, it is labelled as an outlier. This technique is based on the assumption that most correspondences are inliers and follow the same pattern.

The $2 \mathrm{D}$ case is only used when using hierarchical matching to reject outliers after the first iteration, whereas the 3D case is used after fine registration and consensus tracking. Since image patch generation can account for minor deviations in the façade plane, such as small building projections, a 3D-based outlier removal is more robust in such a case. For hierarchical matching, image patches have a lower resolution and a translation between image patches is only approximated. Therefore, 2D-based outlier removal is sufficient. 
Moreover, a technique to track a consensus across multiple panoramic images along the trajectory was implemented. The requirement for this technique, however, is that adjacent MM images share correspondences with the same oblique image. Certainly, this tracking technique requires a 3D-based translation to relate registration results of adjacent MM images due to different scenes and thus façades. Experiments are presented in the next section.

\subsection{Experiments}

This section presents results and discusses multiple experiments with respect to various aspects of the registration algorithm. The MM dataset used in this section consisted of 108 panoramic images and depicts a narrow road in the city centre of Rotterdam, the Netherlands (Figure 4.11).

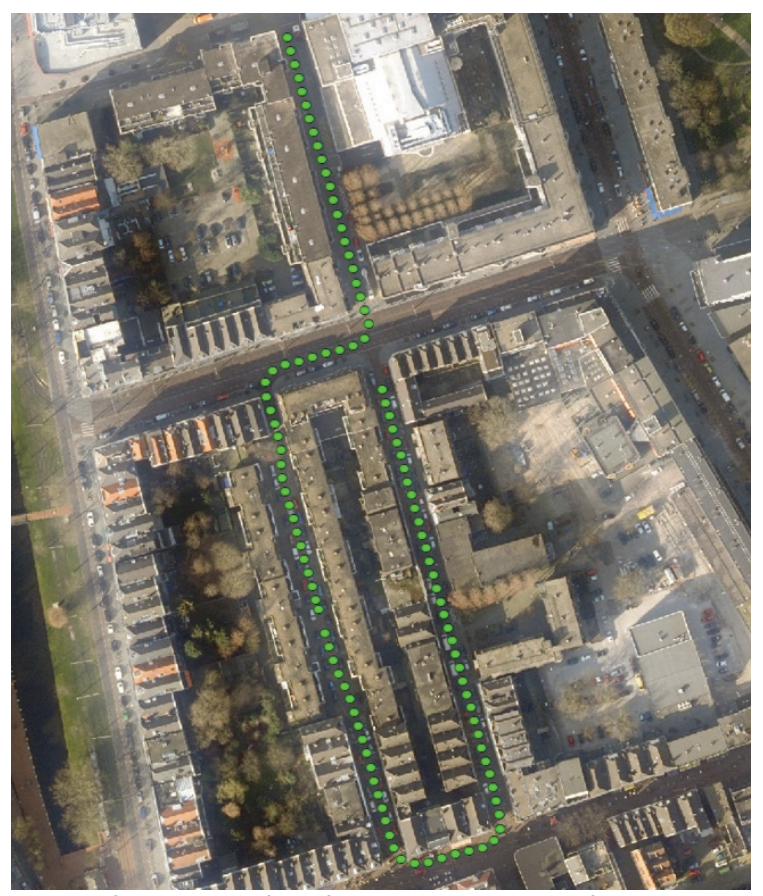

Figure 4.11 Trajectory of MM recording locations in Rotterdam.

The majority of the buildings have three to four storeys and range from about 10 to $20 \mathrm{~m}$ in height. The width of the road is mostly around $10 \mathrm{~m}$, though in some places narrows to $7 \mathrm{~m}$ and widens up to $20 \mathrm{~m}$. The absolute accuracy of the dataset cannot be disclosed due to proprietary knowledge of the data provider. MM panoramic images were recorded every $5 \mathrm{~m}$ along the trajectory. A panoramic image was encoded in a spherical equirectangular projection, where each pixel corresponded to the same angular resolution. An image covered $360^{\circ} \times 180^{\circ}$ degrees with a resolution of $0.075^{\circ} / \mathrm{pixel}$, resulting in an image dimension of $4800 \times 2400$ pixels. 
The entire test area was covered by oblique aerial images, which have been acquired in a pentacam fashion (four oblique plus one nadir images) at an altitude of $450 \mathrm{~m}$ with a ground sample distance (GSD) of 5 to $15 \mathrm{~cm}$. For the procedure presented in this chapter, the nadir images of the pentacam were not used. Depending on the individual MM recording location, two to six oblique aerial images qualified for registration.

The processing was implemented to work in a sequential fashion, treating the left and right parts of the trajectory independently (Figure 4.3 (b)). Later in the adjustment, when correspondences are converted into the original image geometries, both sides of the trajectory are combined. In total, 14 different experiments with different processing parameters were conducted (Table 4-1). Entries in bold in Table 4-1 depict differences from the default setting.

For all the other tables (Table 4-2 to Table 4-8), bold entries represent the best result. In the experiments, four different inlier ratios are provided. The inlier ratios were distinguished in two ways: first, before/after outlier removal; and second, with and without considering non-matchable correspondences.

Table 4-1 Parameters for different experiments. NCC is normalised cross correlation; MI is mutual information. Entries in bold represent differences from the default setting.

\begin{tabular}{cccccc}
\hline Experiment & $\begin{array}{c}\text { Maximum } \\
\text { angular } \\
\text { distance } \\
\left({ }^{\circ}\right)\end{array}$ & $\begin{array}{c}\text { Coarse/fine } \\
\text { registration }\end{array}$ & $\begin{array}{c}\text { Minimum } \\
\text { number } \\
\text { of points } \\
\text { for a } \\
\text { plane }\end{array}$ & $\begin{array}{c}\text { Hierarchical } \\
\text { matching }\end{array}$ & $\begin{array}{c}\text { Patch extent } \\
\text { for } \\
\text { registration } \\
\text { (coarse/fine) } \\
(\mathrm{m})\end{array}$ \\
\hline default & 5 & NCC/MI & 10 & yes & $8 / 6$ \\
1 & $\mathbf{2}$ & NCC/MI & 10 & yes & $8 / 6$ \\
2 & $\mathbf{1 0}$ & NCC/MI & 10 & yes & $8 / 6$ \\
3 & $\mathbf{1 5}$ & NCC/MI & 10 & yes & $8 / 6$ \\
4 & $\mathbf{2 0}$ & NCC/MI & 10 & yes & $8 / 6$ \\
5 & 5 & NCC/MI & $\mathbf{5}$ & yes & $8 / 6$ \\
6 & 5 & NCC/MI & $\mathbf{2 0}$ & yes & $8 / 6$ \\
7 & 5 & NCC/MI & $\mathbf{3 0}$ & yes & $8 / 6$ \\
8 & 5 & MI/MI & 10 & yes & $8 / 6$ \\
9 & 5 & NCC/NCC & 10 & yes & $8 / 6$ \\
10 & 5 & - & 10 & no & $\mathbf{8}$ \\
11 & 5 & - & 10 & no & $\mathbf{1 0}$ \\
12 & 5 & NCC/MI & 10 & yes & $\mathbf{1 0 / 6}$ \\
13 & 5 & - & 10 & no & $\mathbf{1 2}$ \\
14 & 5 & NCC/MI & 10 & yes & $\mathbf{1 2 / 6}$ \\
\hline
\end{tabular}

\subsubsection{Maximum Angular Distance to Reference Vector for Plane Fitting}

The first four experiments ( 1 to 4 in Error! Reference source not found.) focused on the maximum angular distance for plane fitting. The angular 
distance of a plane is defined by the difference of its normal vector to the reference vector in degrees in two dimensions (yaw and pitch). Since the reference vector for plane fitting corresponds to the vector perpendicular to the trajectory, relaxing this parameter enables fitting façades that are not parallel to the trajectory. The results for these four experiments and the default are shown in Figure 4.12.

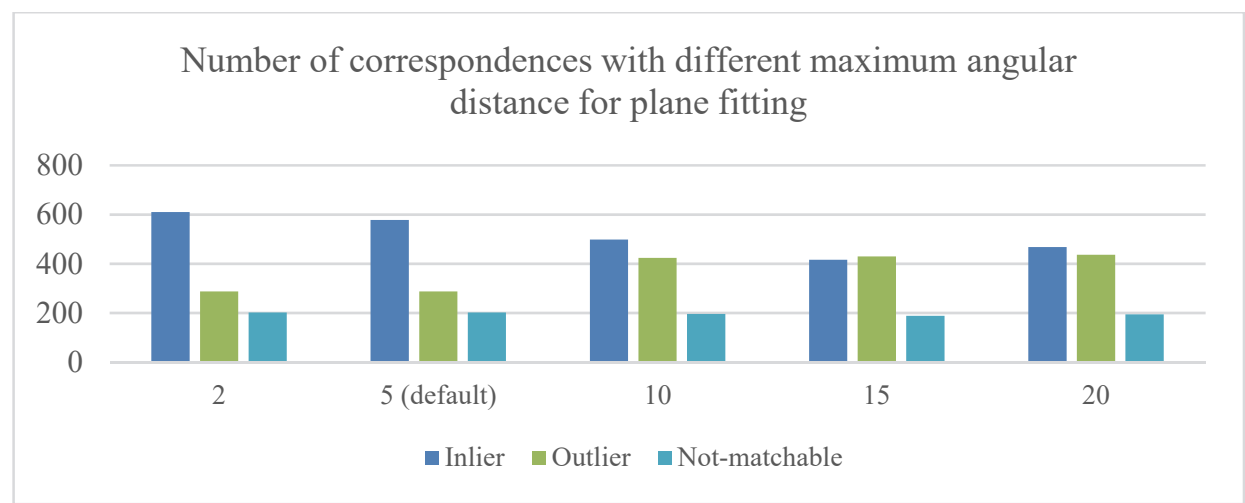

Figure 4.12 Number of correspondences (ordinate) with different maximum angular distance (abscissa) for plane fitting.

Table 4-2 Results for the default and experiments 1 to 4 [different threshold values for maximum angular distance for plane fitting]. Bold entries represent the best result.

\begin{tabular}{|c|c|c|c|c|c|}
\hline Parameter set (see Table I) & default & 1 & 2 & 3 & 4 \\
\hline Maximum angular distance $\left({ }^{\circ}\right)$ & 5 & 2 & 10 & 15 & 20 \\
\hline Inlier percentage & $54 \%$ & $56 \%$ & $45 \%$ & $40 \%$ & $43 \%$ \\
\hline $\begin{array}{l}\text { Inlier percentage without non-matchable } \\
\text { correspondences }\end{array}$ & $67 \%$ & $68 \%$ & $54 \%$ & $50 \%$ & $51 \%$ \\
\hline Inlier percentage after outlier removal & $70 \%$ & $70 \%$ & $47 \%$ & $60 \%$ & $58 \%$ \\
\hline $\begin{array}{l}\text { Inlier percentage after outlier removal without } \\
\text { non-matchables }\end{array}$ & $80 \%$ & $80 \%$ & $54 \%$ & $66 \%$ & $64 \%$ \\
\hline
\end{tabular}

It became evident that relaxing the threshold for a plane's deviation from the reference vector has rather negative consequences on the result, with a decrease of about $20 \%$ with respect to the inlier rate (Table 4-2). This is mostly because the plane does not fit properly to the façade points, which leads to a skewed image patch and thus a wrong registration result (Figure 4.13). Certainly, additional correspondences may be identified which were not possible on the assumption of parallelism between $\mathrm{MM}$ vehicle trajectory and façade surface. If the angular distance is reduced to $2^{\circ}$ (parameter set for experiment 1), a slightly higher number of inliers, compared to the default parameter set, could be identified. Although more inlying correspondences were returned, a stricter threshold reduces the flexibility of the approach. In this case, the default parameter set returned a higher number (four in total) of individual recording locations with direct correspondences to the aerial 
images than parameter set 1 . Moreover, a more tolerant maximum angular distance threshold does not necessarily result in the same set of estimated planes. This is only the case if a façade is represented well by the sparse point cloud.
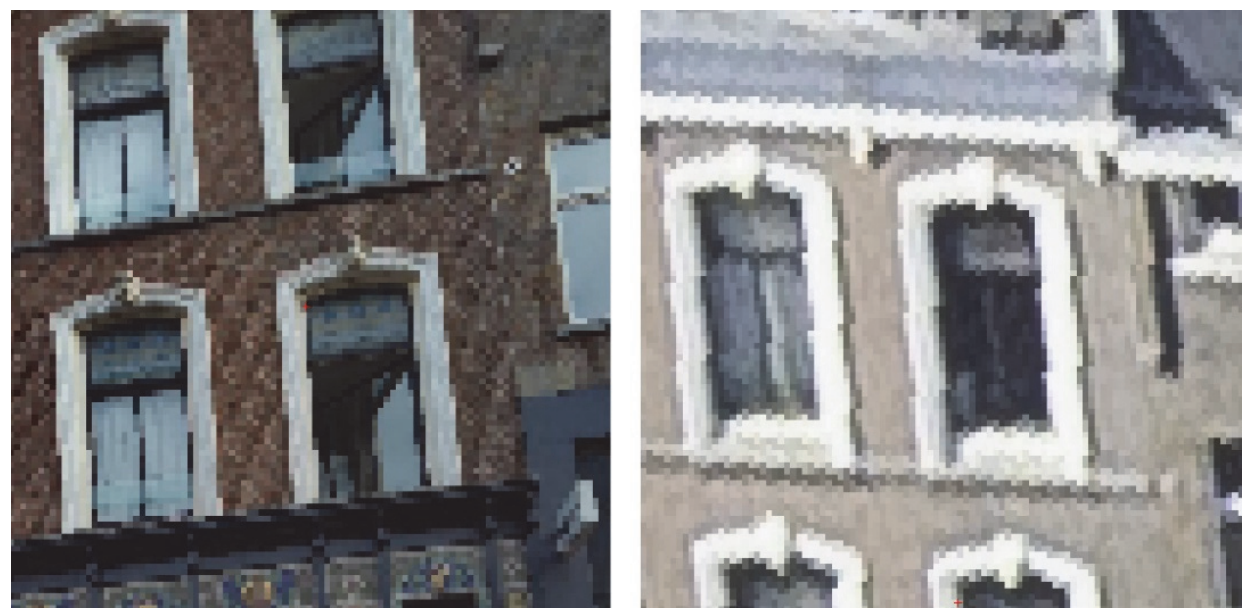

Figure 4.13 Wrong plane estimation leads to skewed image patches.

\subsubsection{Minimum Number of Points for Plane Fitting}

Another threshold in the plane fitting process is to adjust the minimum number of points required for plane fitting (Figure 4.14). This parameter determines whether defined criteria for plane fitting can conform to a required minimum number of points per plane. The number and distribution of points which can be used for plane fitting depends on the actual scene (Figure 4.15). Table 4-4 provides an insight into the absolute number of correspondences used for the plane fitting process. Figure 4.16 depicts the distribution of correspondences across all perspective views.

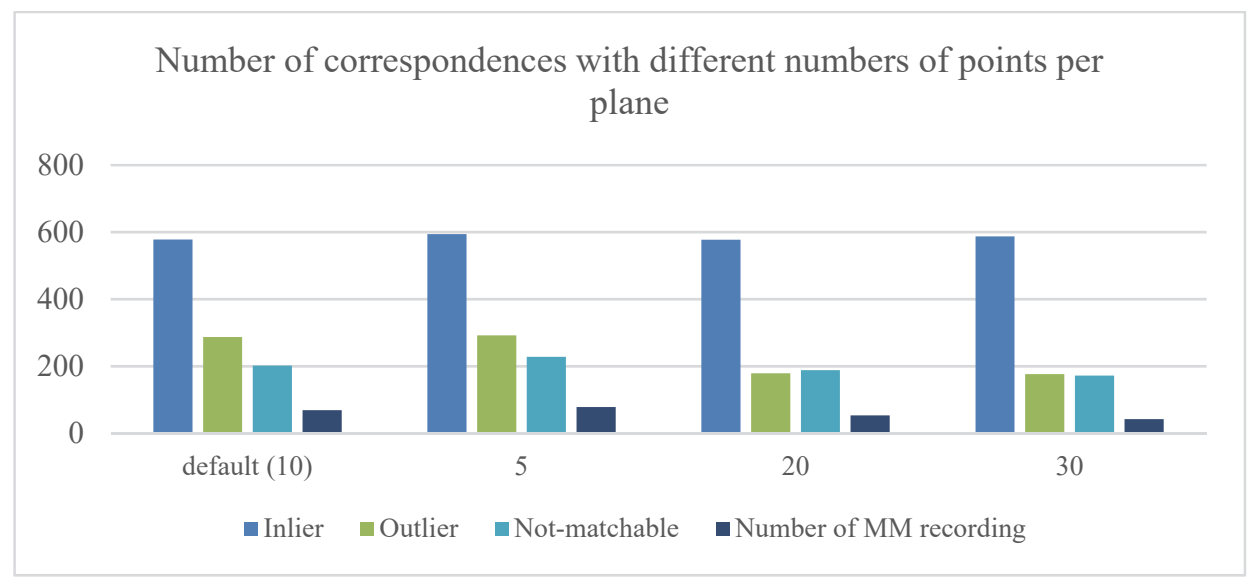

Figure 4.14 Number of correspondences with different number of points per plane. 
Table 4-3 Parameters for the default and results of experiments 5 to 7 [different minimum number of points for plane fitting]. Bold entries represent the best result.

\begin{tabular}{|c|c|c|c|c|}
\hline \multirow{2}{*}{$\begin{array}{l}\text { Parameter set (see Table I) } \\
\text { Minimum number of points per plane }\end{array}$} & default & 5 & 6 & 7 \\
\hline & 10 & 5 & 20 & 30 \\
\hline $\begin{array}{l}\text { Number of MM recordings with correspondences (out of } \\
108 \text { ) }\end{array}$ & 69 & 78 & 53 & 42 \\
\hline Inlier percentage & $54 \%$ & $53 \%$ & $61 \%$ & $63 \%$ \\
\hline $\begin{array}{l}\text { Inlier percentage } \\
\text { correspondences }\end{array}$ & $67 \%$ & $67 \%$ & $76 \%$ & $77 \%$ \\
\hline Inlier percentage after outlier removal & $70 \%$ & $66 \%$ & $70 \%$ & $63 \%$ \\
\hline $\begin{array}{l}\text { Inlier percentage after outlier removal without non- } \\
\text { matchables }\end{array}$ & $80 \%$ & $85 \%$ & $83 \%$ & $78 \%$ \\
\hline
\end{tabular}

The results among different parameter sets are almost identical, returning a similar number of inliers, outliers and non-matchable correspondences. However, the number of recording locations with correspondences decreased with the number of points required for a plane, indicating that some planes along the trajectory are constituted by only 10 or fewer points (see Figure 4.17).

Table 4-4 Statistics of correspondences between perspective views of the entire trajectory.

\begin{tabular}{lcc}
\hline Side of the trajectory & Right & Left \\
\hline \hline Number of correspondences & 7094 & 4050 \\
Median correspondences per view & 22 & 16.5 \\
Standard deviation of correspondences per view & 107.92 & 66.45 \\
\hline
\end{tabular}



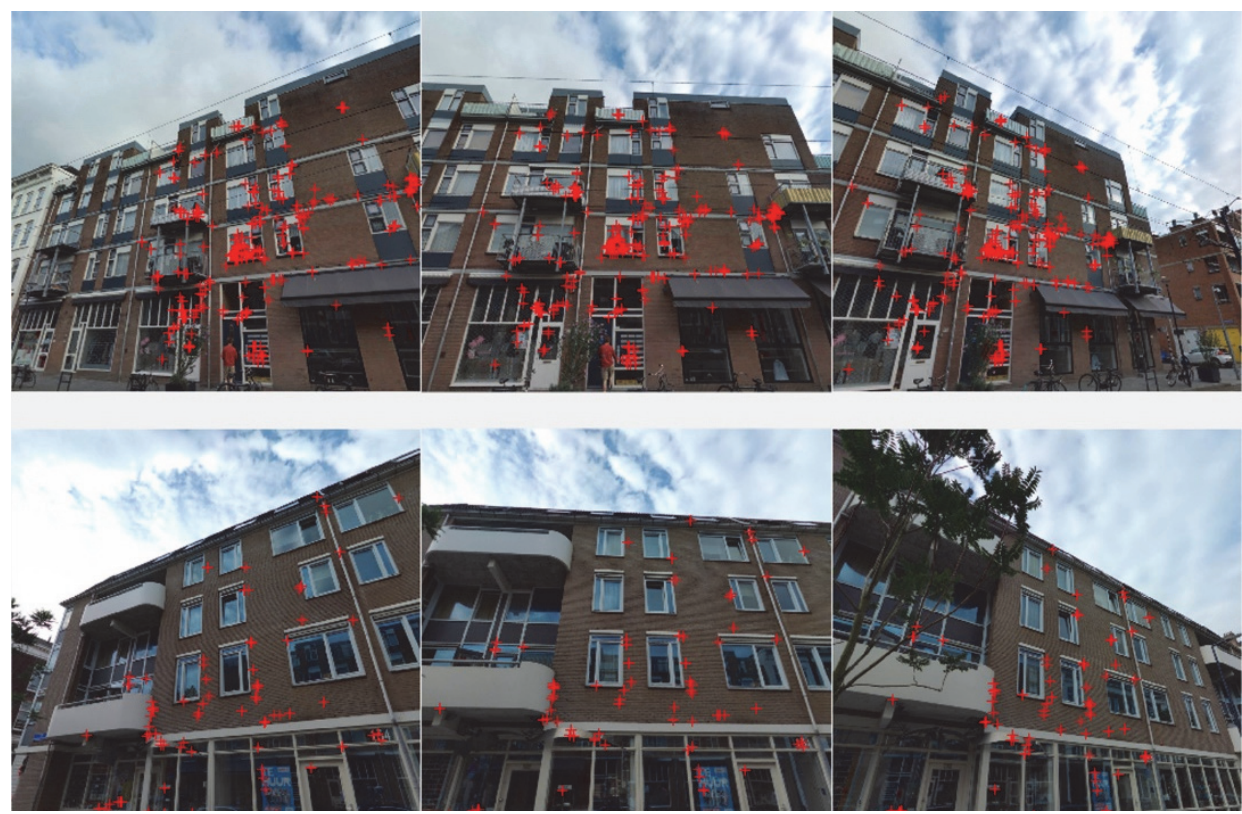

Figure 4.15 Example of correspondences between two triplets of the same location. (Top: right-hand side of trajectory; bottom: left-hand side.)

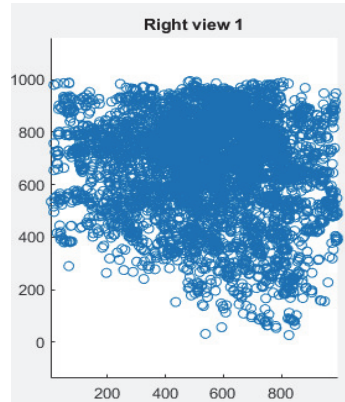

Left view 1

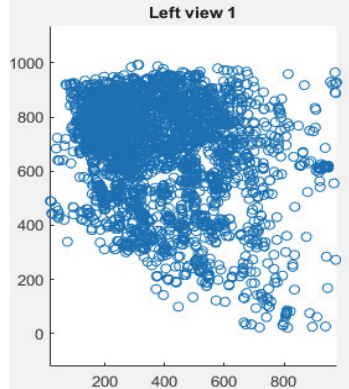

Right view 2

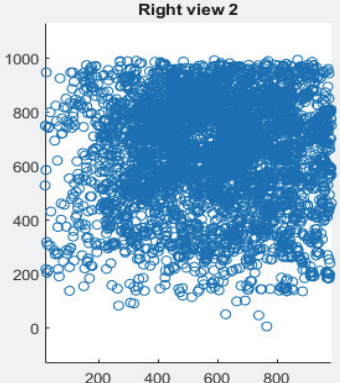

Left view 2

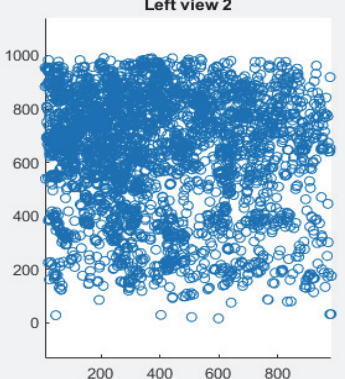

Right view 3

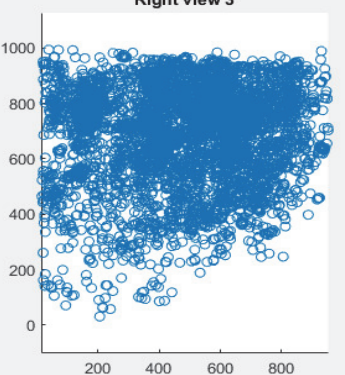

Left view 3

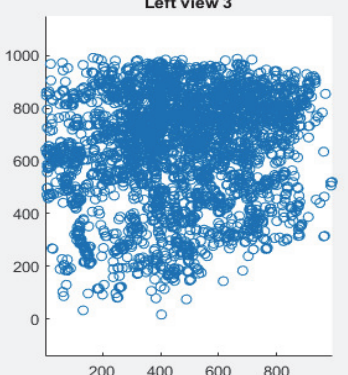

Figure 4.16 Distribution of triplet correspondences across the entire trajectory for both sides. Top row: correspondences on the right-hand side of the trajectory. Bottom row: correspondences on the left-hand side. Note the equal distribution as well as the slanted pattern on the side views representing façades. 
The results among different parameter sets are almost identical, returning a similar number of inliers, outliers and non-matchable correspondences. However, the number of recording locations with correspondences decreased with the number of points required for a plane, indicating that some planes along the trajectory are constituted by only 10 or fewer points (see Figure 4.17).

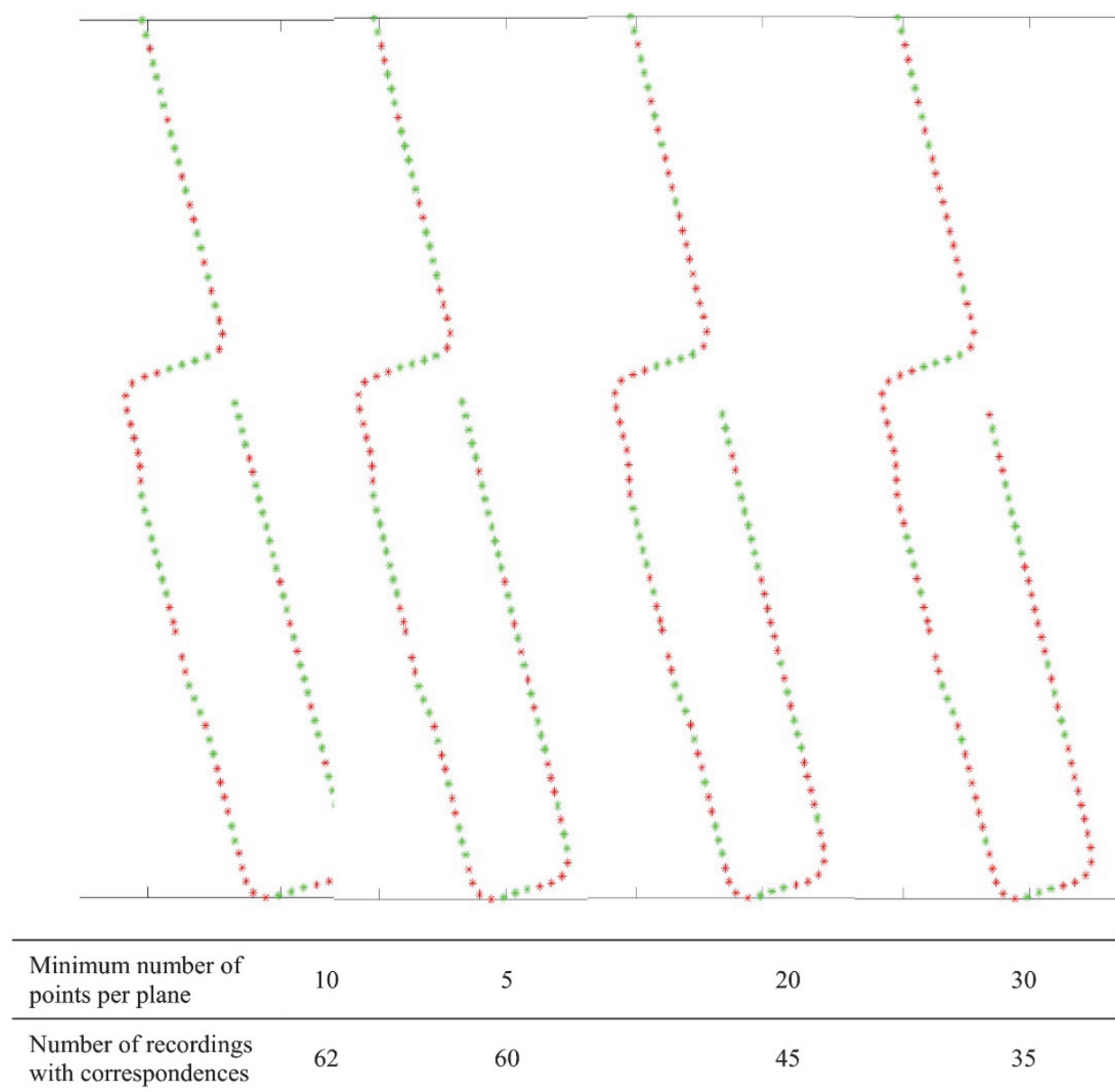

Figure 4.17 Influence on minimum number of points per plane; a comparison of recording locations with (green) and without (red) correspondences. From left to right: 10 points (default), 5 points, 20 points and 30 points. See also Figure 4.11 .

\subsubsection{Mutual Information to Compute Initial Transformation}

By default, hierarchical matching is used to retrieve an estimate of the translation between the image patches by normalised cross correlation. Subsequently, a fine registration computes the precise offset between both patches using mutual information. In this experiment, mutual information is used to obtain an initial transformation instead of normalised cross correlation 
using the same set of parameters as for a subsequent fine registration (see parameter set 8 in Table 4-5). For comparison, the same experiment has been conducted using normalised cross correlation for fine registration (parameter set 9 in Table 4-5).

Table 4-5 Results for the default and experiments 8 and 9 [initial transformation/fine registration with MI or NCC only]. Bold entries represent the best result.

\begin{tabular}{lccc}
\hline Parameter set (see Table I) & default & 8 & 9 \\
Initial transformation & NCC & MI & NCC \\
Fine registration & MI & MI & NCC \\
\hline \hline Inliers & 578 & 421 & $\mathbf{6 7 9}$ \\
$\begin{array}{l}\text { Outliers } \\
\text { Non-matchable correspondences }\end{array}$ & 287 & 1124 & $\mathbf{2 7 1}$ \\
\hline $\begin{array}{l}\text { Inlier percentage } \\
\text { Inlier percentage } \quad \text { without non-matchable }\end{array}$ & 202 & 1347 & $\mathbf{1 8 9}$ \\
$\begin{array}{l}\text { correspondences } \\
\text { Inlier percentage after outlier removal }\end{array}$ & $67 \%$ & $27 \%$ & $\mathbf{7 1 \%}$ \\
$\begin{array}{l}\text { Inlier percentage after outlier removal without non- } \\
\text { matchables }\end{array}$ & $\mathbf{8 0 \%}$ & $18 \%$ & $\mathbf{7 3 \%}$ \\
\hline
\end{tabular}

Although the total number of identified correspondences is higher if mutual information is used for coarse registration, the inlier rate drops significantly. On the other hand, if normalised cross correlation is used for both steps finding an initial transformation as well as fine registration - the results remain comparable to the results with the default parameter set. While normalised cross correlation is suitable for coarse registration and its fine registration capabilities are good, it is not on par with mutual information, which is able to locate correspondences more accurately (Figure 4.18). 

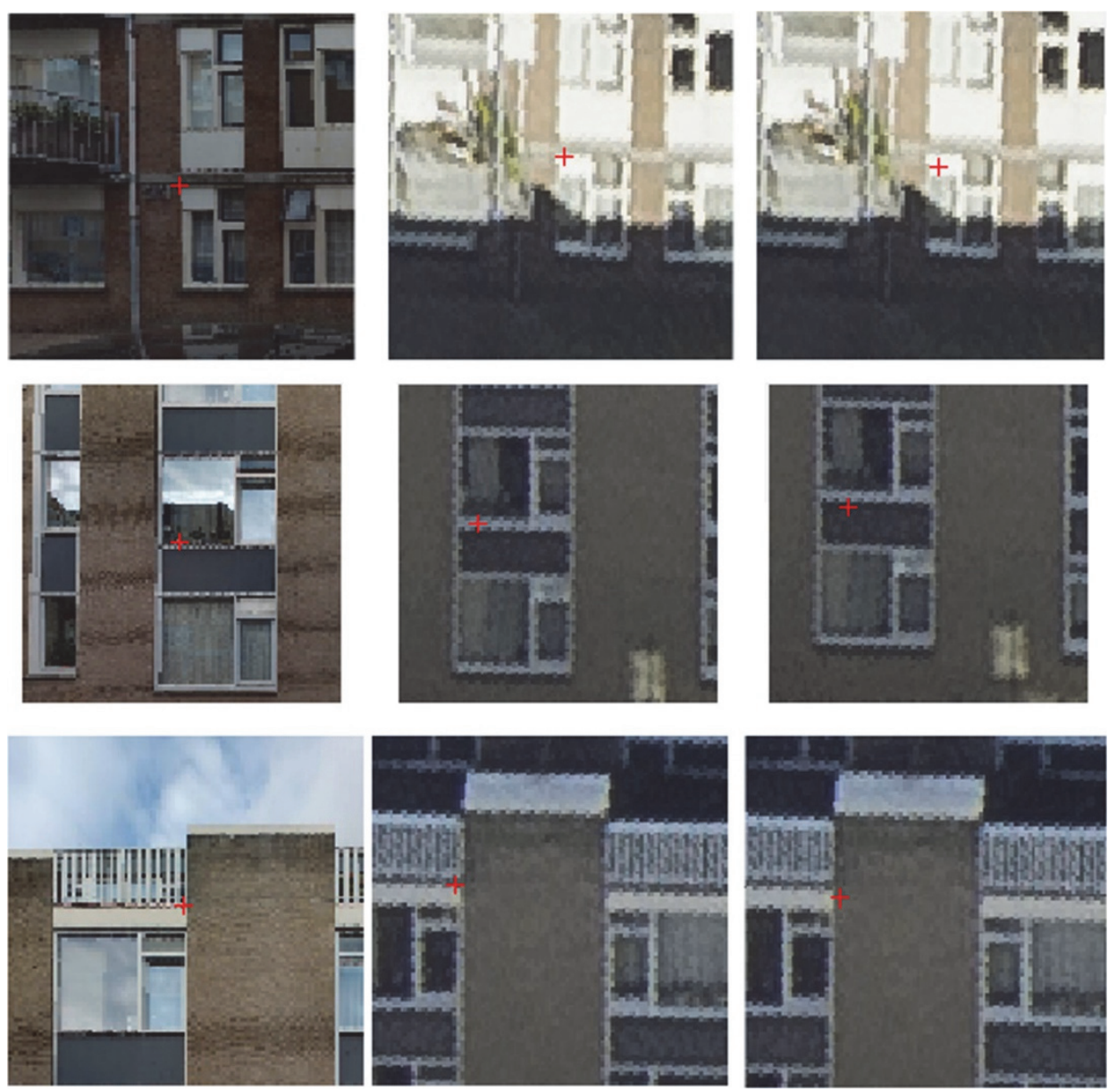

Figure 4.18 Comparison between fine registration results of mutual information and normalised cross correlation for three examples (top, middle, bottom). In each example: left image is the MM patch; middle image is the MI registration result in the oblique patch; right image is the NCC registration result in the oblique patch.

\subsubsection{Hierarchical Matching and Patch Size}

Important properties of the registration procedure are the definition of the image patch size and utilising hierarchical matching. A first iteration derives a coarse registration between the image patches utilising contextual information, whereas a second iteration conducts the fine registration. In this experiment, both properties (patch size and hierarchical matching) are analysed. 


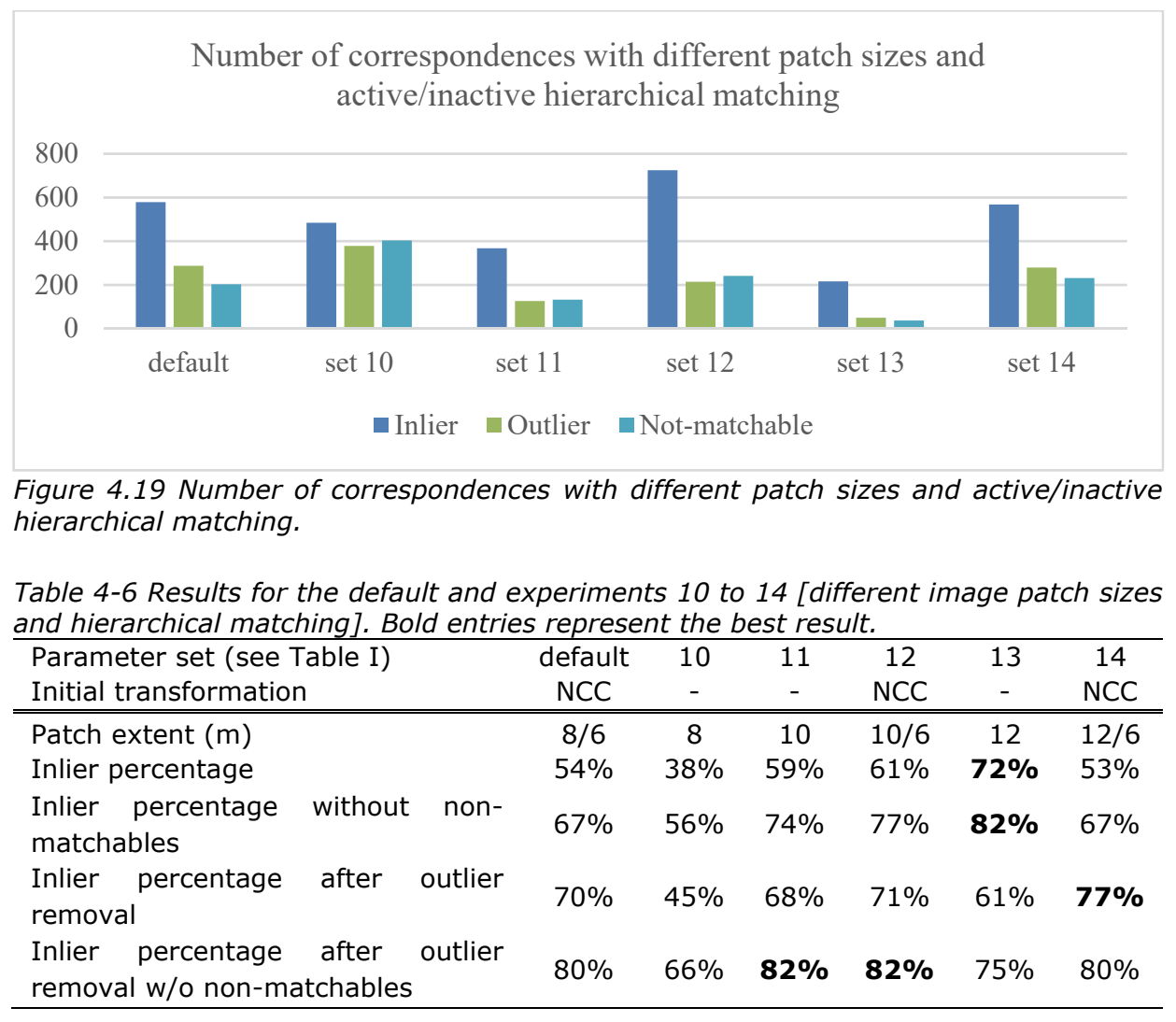

Comparing the results in Table 4-6 and Figure 4.19 of the default parameter set with set 10 (see Table 4-1 for details), it becomes evident that hierarchical matching improves the matching result considerably. The increase in inliers if hierarchical matching (HM) is used is also observable between set 11 (no HM) and set 12 (with HM), as well as between set 13 (no HM) and set 14 (with HM). The relationship between the inlier/outlier ratio is, however, different. Whereas hierarchical matching seems to improve this ratio in the first two set pairs (default and 10), the second set pair (11 and 12) retains the same ratio while the relation in the third set pair (13 and 14) flips (if outlier removal has not been used). The patch size itself also has a great impact on the results. Set 12 returned most inliers, and uses an initial patch size of $10 \mathrm{~m}$. Increasing the patch size to $12 \mathrm{~m}$, however, leads to a reduction in the total number of correspondences. With fewer than a third of the inliers of set 12 , set 13 returns the least number of correspondences, as large patches may involve parts of the scene which are not part of the physical plane (Figure 4.20). 


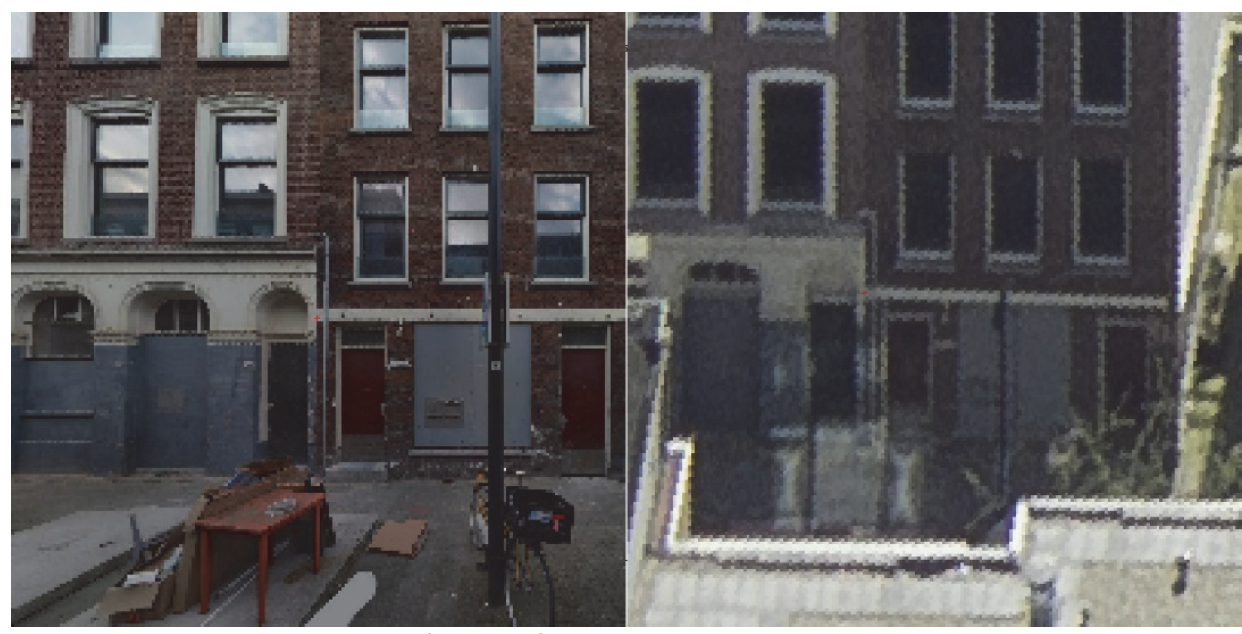

Figure 4.20 Large image patch size of $12 \mathrm{~m}$.

A drawback of larger patches is also related to processing time, as the patch creation method is one the most computationally expensive steps in the procedure. In fact, a patch with $12 \mathrm{~m}$ sides has an area of $144 \mathrm{~m}^{2}$, which is more than twice the area $\left(64 \mathrm{~m}^{2}\right)$ of a patch with $8 \mathrm{~m}$ sides.

\subsubsection{Outlier Removal}

The method to remove outliers is based on the metric (3D case) or pixel (2D case) distance from a median consensus of all correspondences. Table $\underline{7}$ depicts the number of inliers before and after outlier removal for the $3 D$ case. It is apparent that a higher relative number of inliers ( $114 \%$ on average) can be achieved at the expense of removing a large number of correct correspondences ( $67 \%$ on average). In only one instance did the relative number of inliers decrease after outlier removal (set 13), due to a high inlier rate and a low number of correspondences. The ratio between the numbers of inliers before and after outlier removal depicts how many inliers in the original set could be preserved after outlier removal. In some cases (for instance, the default set), fewer than a quarter of the original inlier points remain. The last column of Table 4-7 expresses this relationship as the increase of inliers with respect to the absolute number of correspondences. 
Table 4-7 Comparison of inlier rate before and after outlier removal. Bold entries represent the best result.

\begin{tabular}{ccccccc}
\hline $\begin{array}{c}\text { Experiment } \\
\text { (parameter } \\
\text { Set) }\end{array}$ & $\begin{array}{c}\text { Number } \\
\text { of inliers }\end{array}$ & $\begin{array}{c}\text { As a } \\
\text { percentage } \\
(\%)\end{array}$ & $\begin{array}{c}\text { Number of } \\
\text { inliers } \\
\text { after } \\
\text { outlier } \\
\text { removal }\end{array}$ & $\begin{array}{c}\text { As a } \\
\text { percentage } \\
(\%)\end{array}$ & $\begin{array}{c}\text { Ratio of } \\
\text { number of } \\
\text { inliers } \\
\text { before and } \\
\text { after outlier } \\
\text { removal }\end{array}$ & $\begin{array}{c}\text { Relative } \\
\text { increase } \\
\text { in inlier } \\
\text { ratio } \\
(\%)\end{array}$ \\
\hline \hline Default & 578 & 66.82 & 137 & 79.65 & 23.70 & 119.20 \\
1 & 610 & 68.00 & 163 & 79.51 & 26.72 & 116.92 \\
2 & 498 & 54.01 & 102 & 54.26 & 20.48 & 100.45 \\
3 & 416 & 49.17 & 133 & 66.17 & 31.97 & $\mathbf{1 3 4 . 5 7}$ \\
4 & 468 & 51.77 & 122 & 64.21 & 26.06 & 124.03 \\
5 & 594 & 67.04 & 167 & $\mathbf{8 4 . 7 7}$ & 28.11 & 126.44 \\
6 & 577 & 76.32 & 159 & 82.81 & 27.56 & 108.5 \\
7 & 587 & 76.93 & 153 & 78.06 & 26.06 & 101.47 \\
8 & 421 & 27.25 & 117 & 33.24 & 27.79 & 121.98 \\
9 & 679 & 71.47 & 193 & 78.46 & 28.42 & 109.77 \\
10 & 484 & 56.15 & $\mathbf{2 7 4}$ & 66.34 & 56.61 & 118.16 \\
11 & 367 & 74.44 & 232 & 81.69 & 63.22 & 109.74 \\
12 & $\mathbf{7 2 4}$ & 77.19 & 168 & 81.55 & 23.2 & 105.66 \\
13 & 216 & $\mathbf{8 1 . 5 1}$ & 135 & 75.00 & 62.5 & 92.01 \\
14 & 568 & 67.06 & 142 & 79.78 & $\mathbf{7 5 . 0 0}$ & 118.96 \\
\hline
\end{tabular}

A rejection threshold defines if a set of correspondences conforms to the consensus. A default of $0.5 \mathrm{~m}$ (in 3D space) has been selected for the experiments above. Table 4-8 depicts different rejection thresholds for the default parameter set. Certainly, the number of inliers increases with a more tolerant rejection threshold (1.0) while it decreases with a less tolerant threshold (0.3). However, the inlier ratio will decrease if the rejection threshold is too tolerant.

Moreover, the last column in Table 4-8 represents a result with consensustracking activated. In this case, a median consensus of adjacent MM images sharing correspondences with the same oblique image can be tracked. This technique is, however, only useful if rather long, uninterrupted stretches of MM images are present which share tie information with the same oblique image. In the test dataset, this was only the case with a maximum sequence of five MM images. Hence, this technique will have to prove itself to be useful in future experiments.

Table 4-8 Rejection threshold for default parameter set. The last column shows results with consensus-tracking activated. Bold entries represent the best result.

\begin{tabular}{lcccc}
\hline Rejection threshold & 0.5 (default) & 1 & 0.3 & 0.5 (tracking) \\
\hline \hline Number of inliers after outlier removal & 137 & $\mathbf{1 7 3}$ & 101 & 145 \\
Number of outliers & 35 & 81 & $\mathbf{2 1}$ & 41 \\
In percent & 79.65 & 68.11 & $\mathbf{8 2 . 7 9}$ & 77.96 \\
\hline
\end{tabular}




\subsection{Discussion and Conclusion}

This chapter has presented a fully automatic procedure to register panoramic MM images with oblique aerial imagery. An array of different techniques and heuristics is needed to retrieve accurate correspondences. Based on mutual planes in object space, both image datasets can be homogenised to allow for a registration at pixel-level accuracy.

As far as the preprocessing steps are concerned, it became evident that the influence of plane fitting is very significant on the entire outcome of the procedure. For example, the parameter "minimum number of points per plane" translates to a constraint, which can reduce the number of outliers at the expense of façades to be integrated into the process. In the "Experiments" section, all results have been compared to the outcome using the default parameter set, which the authors consider best practice. Currently, parameters are defined such as an occlusion threshold, a maximum distance of a point to a plane, a maximum angular distance to the reference vector and a minimum number of points contributing to a plane. For instance, the size of a plane, the distribution of points constituting it and a tracking of planes across multiple images, could further improve the detection rate. Certainly, the integration of additional parameters may complicate the procedure.

Another interesting property of the procedure is the patch size. The higher its value, the larger the grid will become. Choosing the right size for a patch is of great significance, as a template registration technique is used. If the grid is too small, lack of context will impede the registration; if the grid is too large, it may project above the actual plane in object space, leading to distortions. In the "Experiments" section, it is apparent that a large patch size returns a higher inlier rate, as the registration becomes more stable. This requires, however, that the scene is depicted similarly in both datasets, as the registration would not converge otherwise. Hierarchical matching takes advantage of this and can improve the registration result considerably. Although, an iterative hierarchical matching with more than two iterations seems a logical continuation, additional computational costs due to the recomputation of patches with different sampling rates, as well as the nature of template matching, may render additional iterations superfluous. Further endeavours should focus on utilising registration results from previous recording locations to constrain the search space and enable implicit quality control of correspondences.

With this idea in mind, an outlier removal technique which is able to track consensus has been presented. Although it returns many false negatives in the procedure, it increases the inlier rate in almost every case. Moreover, it is prone to converge incorrectly if the majority of correspondences are false. Thus, an interesting research question is whether a robust bundle adjustment, 
which includes outlier detection as well, will perform better with or without previous outlier filtering. In general, the procedure works reliably in this nonstandard registration scenario. The experiments have shown that, even in difficult scenarios, an inlier rate of $80 \%$ is achievable. Future efforts will be directed towards the integration of this procedure into an adjustment solution, as well as to further increase the flexibility of the approach to reduce false positives. 


\section{Chapter 5 - Correction of mobile mapping trajectories in GNSS-denied environments using aerial nadir and aerial oblique images ${ }^{20}$}

20 This chapter is based on:

Fanta-Jende, P., Nex, F., Gerke, M., Lijnen, J., \& Vosselman, G. (2019) 'Correction of mobile mapping trajectories in GNSS-denied environments using aerial nadir and aerial oblique images, ISPRS Archives; Vol. XLII-2/W13, https://doi.org/10.5194/isprsarchives-XLII-2-W13-1649-2019

Please note: additional experiments have been conducted with respect to different adjustment scenarios which can be found in the annex to this chapter (see section 5.7) 


\subsection{Abstract}

Mobile mapping enables highly accurate as well as high-resolution image data capture at low cost and high speed. As a terrestrial acquisition technique predominately employed in urban, and thus built-up areas, non-line-of-sight and multipath effects challenge its absolute positioning capabilities provided by GNSS. In conjunction with IMU drift, the platform's trajectory has an unknown accuracy, which influences the quality of the data product. By employing a highly accurate co-registration technique for identifying tie correspondences between mobile mapping images and aerial nadir as well as aerial oblique images, reliable ground control can be introduced into an adjustment solution. We exemplify the performance of our registration results by showcasing adjusted mobile mapping trajectories in four different test areas, each with about 100 consecutive recording locations (approx. $500 \mathrm{~m}$ length) in the city centre of Rotterdam, The Netherlands. The mobile mapping data has been adjusted in different configurations, i.e. with nadir or oblique aerial correspondences only and if possible in conjunction. To compare the horizontal as well as the vertical accuracy before and after the respective adjustments, more than 30 ground control points were surveyed for these experiments. In general, the aim of our technique is not only to correct mobile mapping trajectories in an automated fashion but also to verify their accuracy without the need to acquire ground control points. In most of our test cases, the overall accuracy of the mobile mapping image positions in the trajectory could be improved. Depending on the test area, an RMSE in 3D between 15 and $21 \mathrm{~cm}$ and an RMSE in 2D between 11 and $18 \mathrm{~cm}$ is achievable. 


\subsection{Introduction}

As a terrestrial geo-data acquisition platform, mobile mapping is affected by GNSS-induced positioning issues, such as multipath or non-line-of-sight effects. Consequently, the absolute position of the platform cannot be reliably determined at all times and acquired data postings' accuracy is unknown and likely impaired. A correction of the platform's trajectory or alternatively the data product is usually conducted by introducing external references, such as ground control points (GCPs) or digital map data. Whereas GCPs offer a high accuracy but are labour-intensive to acquire and to integrate, maps are generalisations of the real world, difficult to intersect with acquired MM data, and cannot necessarily provide for surveying-grade accuracy. In our previous work, we have presented co-registration approaches for mobile mapping and aerial nadir and oblique images (Fanta-Jende et al., 2019; Jende et al., 2018a; Jende et al., 2018b). Airborne platforms are not affected by the aforementioned GNSS issues, and aerial images can thus be used as a reference. The major challenge is to overcome the large perspective differences to identify mutual features in the terrestrial and aerial data set. Nadir and oblique aerial images have different properties as well; hence, two different co-registration approaches with the mobile mapping images have been devised. Both approaches are briefly discussed in the methodology section (5.4.1 and 5.4.2). After the identification of correspondences between the terrestrial and aerial data set, multiple adjustment options are possible. For the experiments in this section, only tie points that are at least visible in two aerial images have been used for data adjustment.

\subsection{Related Work}

Approaches to improve terrestrial data are manifold in their characteristics. For instance, distinctions can be made on the real-time or post-processing property of an approach, whether the correction procedure relies on improving the platform's data directly or utilises external data as a reference.

In our case, comparable procedures, i.e. relying on the introduction of an external reference for data adjustment, have been developed by various authors. Cheng et al. (2015) introduce a hierarchical registration approach between mobile and aerial laser scanning point clouds relying on the extraction of building contours and road features. Although no GCPs have been used to evaluate the absolute accuracy of the result, the relative mean accuracy between both data sets after registration is around half a metre in the vertical and horizontal dimension. Gruen et al. (2013) present an approach to combine UAV with MM point clouds for the generation of complete 3D representations. Therefore, both point clouds have been merged using manually measured control points. The relative accuracy between both data sets has been assessed by check points and reaches an RMSE of $11 \mathrm{~cm}$ horizontally and $20 \mathrm{~cm}$ in 
height. Similarly, Molina et al. (2017) developed a tightly-coupled positioning system for the simultaneous acquisition of UAV and ground-based images. The UAV is following an optical target installed on the roof of the terrestrial platform during the acquisition. Depending on the configuration, either the UAV's positioning is used for the correction of the terrestrial platform or the other way around. Planimetric as well as a vertical accuracy below $10 \mathrm{~cm}$ is well achievable with this concept.

Tournaire et al. (2006) suggest utilising zebra crossings for the registration of MM stereo data and aerial imagery. To this end, zebra crossings are extracted with a knowledge-based template approach. Although a thorough statistical setup is missing, the authors claim to have reached sub-decimetre accuracy.

Ji et al. (2015) use correspondences between aerial ortho images and MM images for the estimation of the MM platform's trajectory. Since the search for correspondences is per se noisy and returns many false positives, the authors employ a particle filter solution to estimate the optimal trajectory. The authors report a horizontal accuracy of about $1.0 \mathrm{~m}$ and a vertical accuracy of about $1.30 \mathrm{~m}$ if areas without valid correspondences are truncated.

Hussnain et al. (2018) developed an approach to correct mobile laser scanning platform trajectories by the introduction of correspondences to aerial nadir images. By integrating these correspondences as well as IMU readings into a B-spline estimation, the adjusted trajectories achieve an accuracy (RMSE) of 9 $\mathrm{cm}$ in $X, 14 \mathrm{~cm}$ in $Y$, and $14 \mathrm{~cm}$ in $Z$.

Javanmardi et al. (2017) also utilise correspondences between mobile laser scanning and aerial images to correct the terrestrial data set. The authors report an average error of $11 \mathrm{~cm}$.

In our previous work (Jende et al., 2018a), we analysed the improvement of MM images compared to surveyed GCPs in three test areas using solely correspondences to aerial nadir images. Although a planimetric accuracy of up to $6 \mathrm{~cm}$ has been reached, in some case, an error in height has been introduced due to a very high altitude of the aerial platform ( 4500 metres). This led to glancing intersections due to a bad intersection geometry and consequently an uncertainty in height.

In this chapter, correspondences to aerial oblique and nadir images are used standalone and in conjunction with each other to correct the MM platform's trajectory. 


\subsection{Methodology}

As mentioned earlier, the major challenge of employing aerial images as a reference data set for $\mathrm{MM}$ trajectory correction is a reliable co-registration technique. In our case, MM images are encoded in an equirectangular projection covering 360 degrees horizontally and 180 degrees vertically. This projection entails severe distortions compared to an (aerial) perspective image. Hence, MM images need to be remapped to increase the resemblance and thus the geometric similarity to the aerial images to simplify or rather enable a reliable registration of the data sets. As aerial nadir and oblique images depict a scene from different angles, the perspective remapping has to account for these individual properties. To this end, two different coregistration techniques, one between $\mathrm{MM}$ and aerial nadir images and one between MM and aerial oblique images, have been developed.

\subsubsection{Co-registration of aerial nadir and mobile mapping images}

Aerial nadir images feature a top-down view on the scene. Hence, common entities that are visible in both, the aerial and MM data set, are mostly roads. To this end, MM images are quasi-ortho projected by assuming a ground plane directly located beneath the platform (see Figure 5.1).

The registration is based on corner detection in conjunction with phasecorrelation. Since the data sets are not necessarily perfectly aligned but are displaced within an expected maximum error, orientation parameters are used to constrain the search space for correspondences. With a very high inlier rate of more than 95\%, this technique proved to be successful. For more information, please see Jende et al. (2018a) or 'Chapter 3 Chapter 3 - A fully automatic approach to register mobile mapping and airborne imagery to support the correction of platform trajectories in GNSS-denied urban areas'. 


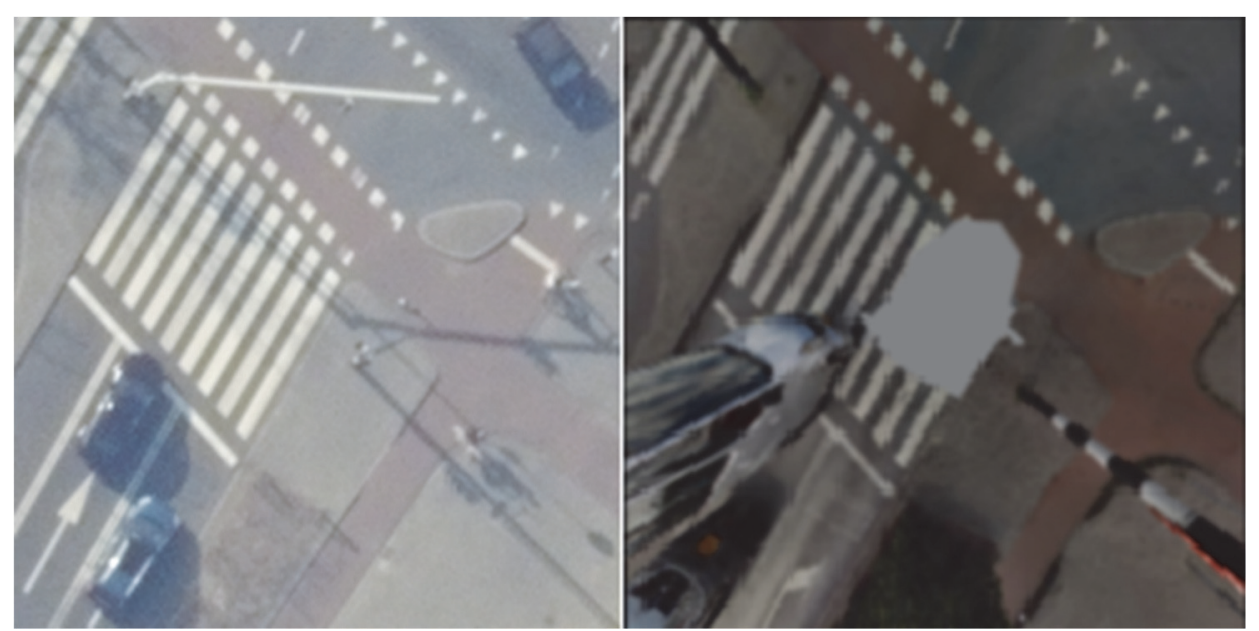

Figure 5.1 Left: Aerial nadir image, right: re-projected MM image.

\subsubsection{Co-registration of aerial oblique and mobile mapping images}

The ground is a mutual geometric surface in object space of aerial nadir and MM images. While the ground is easy to detect or rather assumed and to be utilised as a projection surface for the registration scenario earlier, this relationship cannot be exploited directly for aerial oblique and MM images.

Building façades and other vertical planar structures along the road, however, are visible in the aerial oblique as well as MM image data set. Since the MM images are linked for data adjustment using visual odometry, a sparse point cloud can be generated from these correspondences. Façades can now be detected by a highly constrained plane fitting approach. Extruding inlying sparse points on a façade to a patch allows for the discretisation of an artificial surface in object space. These patches are used to extract image information of $\mathrm{MM}$ as well as aerial oblique images (see Figure 5.2). 

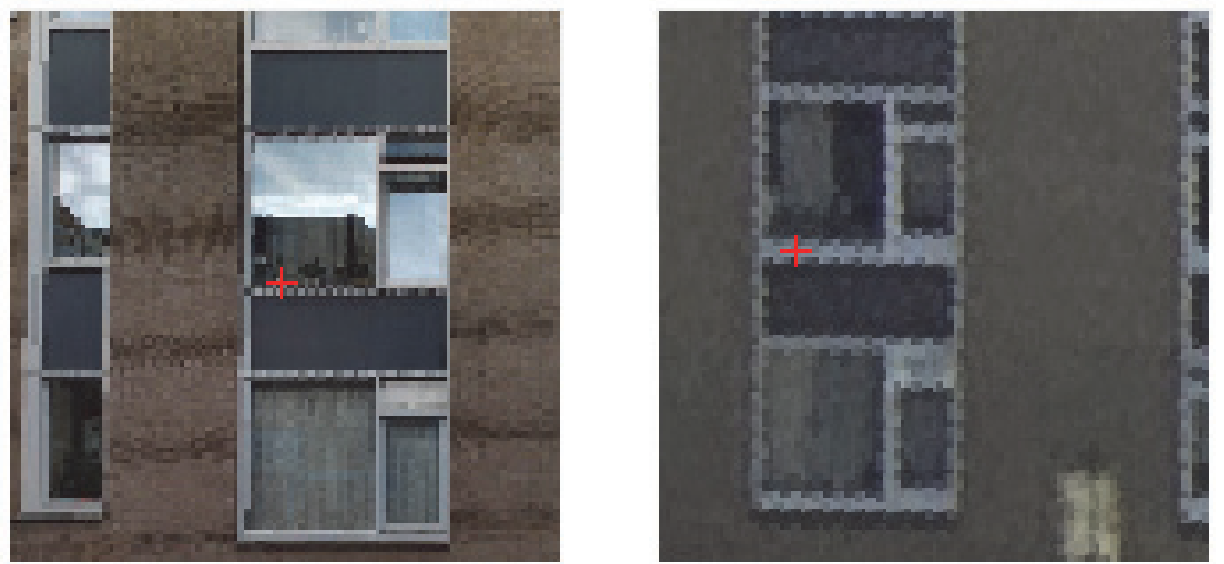

Figure 5.2 Left: Mobile mapping image patch, right: aerial oblique image patch.

Both patches are registered using mutual information. The inlier rate of this technique is about $80 \%$. For further details on this approach, please see FantaJende et al. (2019); Jende et al. (2018b), or Chapter 4 Co-registration of panoramic mobile mapping images and oblique aerial images.

\subsubsection{Mobile mapping data adjustment}

Both co-registration pipelines use projection surfaces to register MM with aerial images. Although the matching is performed locally on geometrically modified images, the transformations are known and the correspondences can be translated into their original geometry.

In order to adjust MM images that do not have direct correspondences to the aerial images, $M M$ images are linked with each other using a visual odometry/structure from motion approach. Although a classical bundle adjustment by locking the exterior orientation of the aerial cameras while the MM recording locations are to be adjusted is possible, the experiments in this section are based on an adjustment where the image observations of the aerial images are triangulated and used as GCPs. Therefore, only correspondences, which are visible in at least two aerial images, are used (see Figure 5.3). The GCPs are all weighted with $10 \mathrm{~cm}$ standard deviation, corresponding to 1 pixel in average in the aerial nadir and oblique image. Future experiments will focus on different and more flexible adjustment scenarios also with respect to the weighting strategy. 


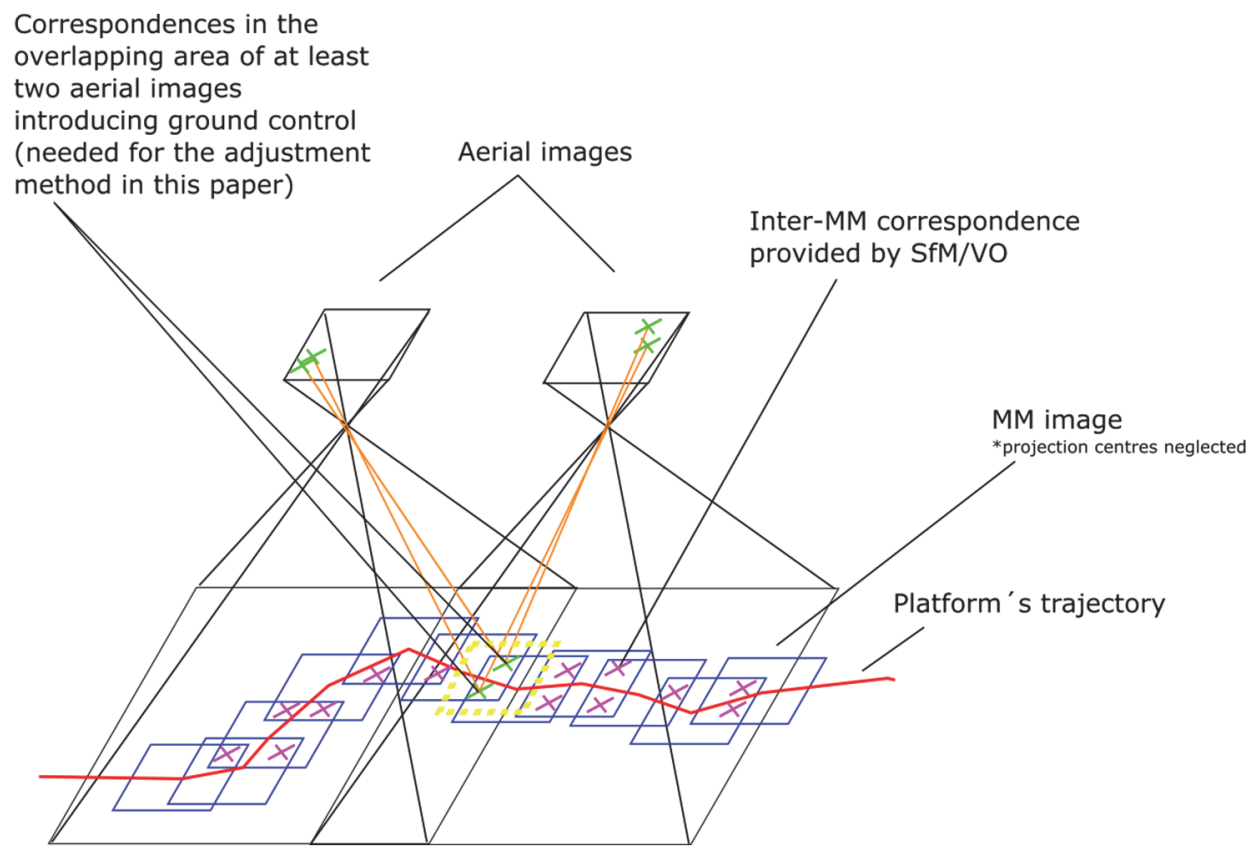

Figure 5.3 Schematics of the selected adjustment method.

\subsection{Experiments}

To ascertain the performance of our registration approaches in different scenarios, four trajectories in Rotterdam, The Netherlands, have been selected. The aerial nadir images in this experiment were acquired at an altitude of 4470 $\mathrm{m}$ and have a ground sampling distance of about $10 \mathrm{~cm}$. Similarly to the experiments conducted in our previous publication (Jende et al., 2018a), this may lead to an uncertainty in height. The aerial oblique images were acquired in a pentacam-fashion at an altitude of $450 \mathrm{~m}$ and have a ground sampling distance ranging from 5 to $15 \mathrm{~cm}$. The test areas vary in their characteristics ranging from open to narrow roads or surrounded by buildings on both or only one side (see Figure 5.4).

Table 5-1 gives an overview on the test areas. For area 3 (blue trajectory in Figure 5.4), only oblique correspondences were used, as the road does not have any salient road markings, hence almost no reliable correspondences to the aerial nadir images could be identified and were thus discarded. Similarly, area 4 (yellow trajectory in Figure 5.4) only features façades that are partially occluded by vegetation or relatively far away from the platform's trajectory. This impedes the plane fitting and the reprojection process, which are pivotal to create image patches from both data sets. Thus, only a few correspondences to the aerial oblique images were returned and were discarded for these 
experiments. In total, 34 GCPs are used as check points to assess the accuracy of the adjusted recording locations.

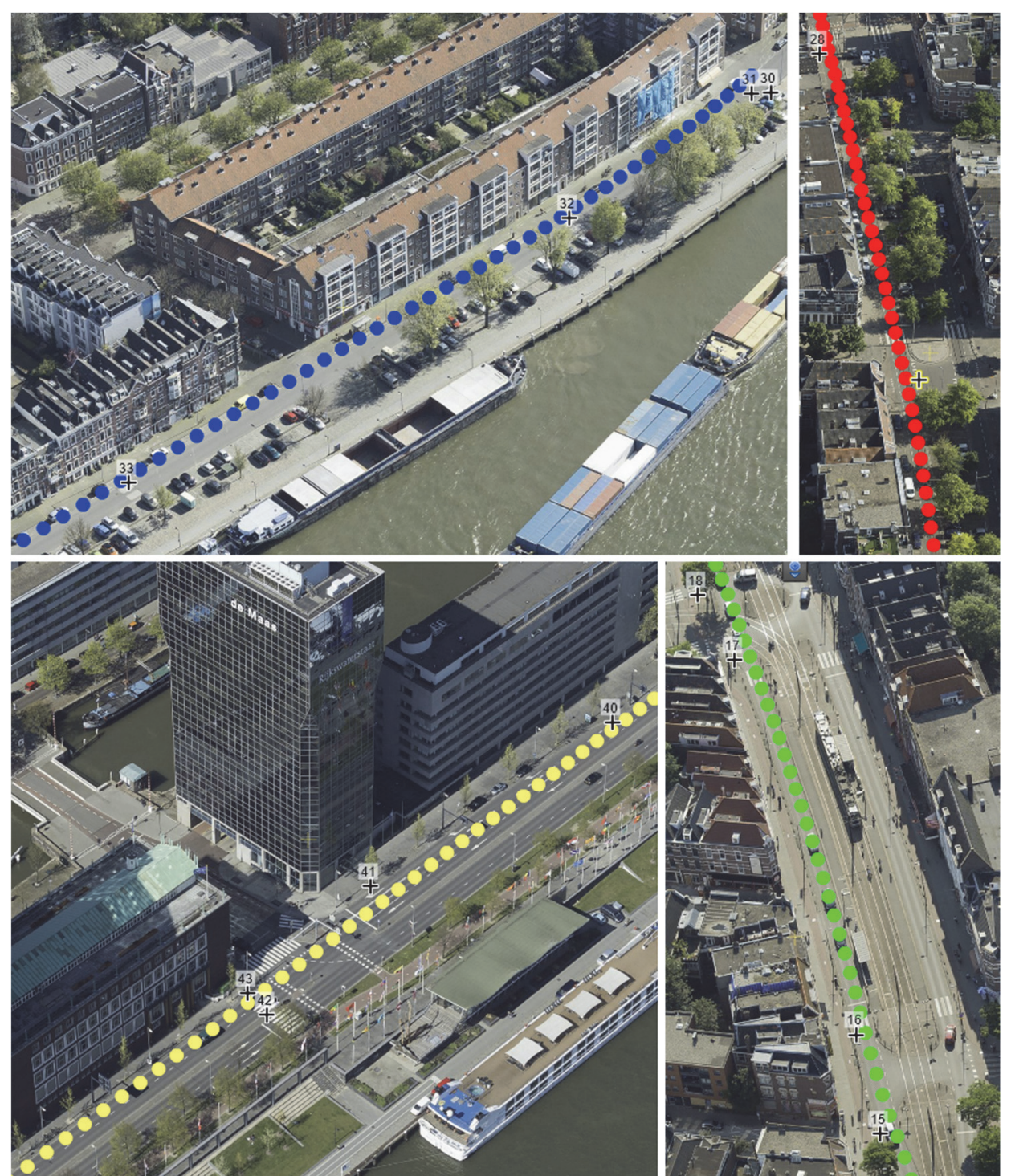

Figure 5.4 Characteristics of the four test areas (only subsets). Area 1 (green trajectory), area 2 (red traj.), area 3 (blue traj.), area 4 (yellow traj.). The recording locations and surveyed GCPs in the selected subset have been projected into an overlapping aerial oblique image. 
Table 5-1 Overview of the four test areas.

\begin{tabular}{ccccc|c}
\hline Area & \multicolumn{2}{c}{$\begin{array}{c}\text { Number of tie } \\
\text { points }\end{array}$} & $\begin{array}{c}\text { Number of MM } \\
\text { images }\end{array}$ & $\begin{array}{c}\text { Joint } \\
\text { adjustment }\end{array}$ & $\begin{array}{c}\text { Number check } \\
\text { points }\end{array}$ \\
\hline \hline 1 & 81 & 68 & 140 & yes & 11 \\
2 & 26 & 29 & 87 & yes & 4 \\
3 & 0 & 95 & 88 & no & 8 \\
4 & 92 & 0 & 150 & no & 11 \\
\hline
\end{tabular}

\subsubsection{Adjustment results using correspondences to the aerial nadir images}

Area 1, 2, and 4 can be adjusted with nadir correspondences only.

Table 5-2 Adjustment result using only correspondences to the aerial nadir images [in metres]. Best result in bold.

\begin{tabular}{lcccccc}
\hline Area & \multicolumn{2}{c}{ RMSE X } & \multicolumn{2}{c}{ RMSE Y } & \multicolumn{2}{c}{ RMSE Z } \\
& before & after & before & after & before & after \\
\hline \hline 1 & 0.14 & $\mathbf{0 . 1 2}$ & 0.12 & $\mathbf{0 . 1 1}$ & 0.23 & $\mathbf{0 . 2 1}$ \\
\hline 2 & $\mathbf{0 . 1 5}$ & 0.21 & $\mathbf{0 . 5 2}$ & 0.53 & $\mathbf{0 . 1 9}$ & 0.23 \\
\hline 4 & 0.19 & 0.19 & 0.16 & $\mathbf{0 . 1 4}$ & $\mathbf{0 . 2 0}$ & 0.24 \\
\hline
\end{tabular}

As mentioned earlier, glancing intersections due to the high altitude of the aircraft may have a negative impact on the vertical component after the adjustment. This effect introduced a larger error in height in the area 2 and 4.

In the case of area 2, utilising only correspondences to the aerial nadir data set had an overall negative impact on the accuracy of the trajectory. First, area 2 has a lower accuracy than the other trajectories and secondly the unequal distribution of correspondences may have had an impact on the adjustment (see Figure 5.5).

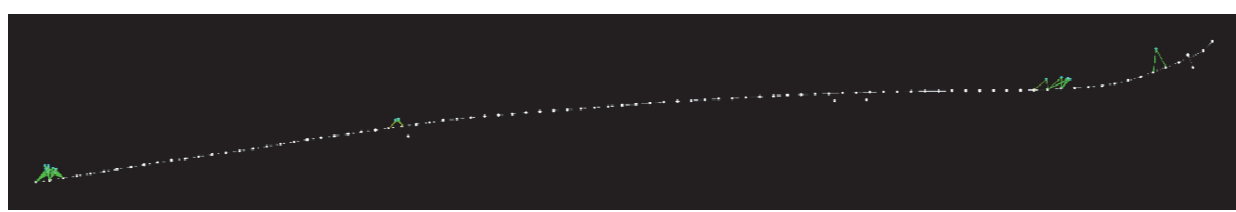

Figure 5.5 Distribution of correspondences (green) of area 2 along the trajectory (white); [rotated by 90 degrees].

Area 4 only shows marginal updates after the adjustment. However, some check points along the trajectory ascertain a more significant update especially in the $Y$ direction (see Table 5-3). Designing an experiment with different weights for the correspondences to the aerial images may better explain this behaviour. 
Table 5-3 Exemplary trajectory updates at two check points in area 4. Best result in bold [in metres].

\begin{tabular}{lcccc} 
& \multicolumn{2}{c}{ CP 39 } & \multicolumn{2}{c}{ CP 43 } \\
& before & after & before & after \\
\hline \hline $\mathrm{X}$ & $\mathbf{0 . 0 2}$ & 0.06 & 0.15 & $\mathbf{0 . 1 4}$ \\
\hline $\mathrm{Y}$ & $\mathbf{- 0 . 1 0}$ & -0.15 & -0.21 & $\mathbf{- 0 . 1 2}$ \\
\hline $\mathrm{Z}$ & 0.22 & $\mathbf{0 . 1 7}$ & $\mathbf{0 . 0 7}$ & 0.12 \\
\hline
\end{tabular}

Area 1 shows minor improvements in all three dimensions and decreases the RMSE in 3D by almost $2 \mathrm{~cm}$ (see Table 5-8). Although this update is marginal, it exemplifies that the registration procedure can be also utilised to verify the accuracy of mobile mapping trajectories by determining the magnitude of an update.

\subsubsection{Adjustment results using correspondences to the aerial oblique images}

Similar to the previous section, three test areas could be processed with using only correspondences to the aerial oblique images.

Table 5-4 Adjustment result using only correspondences to the aerial oblique images [in metres]. Best result in bold.

\begin{tabular}{lcccccc}
\hline \multirow{2}{*}{ Area } & \multicolumn{2}{c}{ RMSE X } & \multicolumn{2}{c}{ RMSE Y } & \multicolumn{2}{c}{ RMSE Z } \\
& before & after & before & after & before & after \\
\hline \hline 1 & 0.14 & $\mathbf{0 . 1 3}$ & $\mathbf{0 . 1 2}$ & 0.14 & 0.23 & 0.23 \\
\hline 2 & $\mathbf{0 . 1 5}$ & 0.21 & 0.52 & $\mathbf{0 . 1 3}$ & $\mathbf{0 . 1 9}$ & 0.28 \\
\hline 3 & $\mathbf{0 . 1 2}$ & 0.12 & 0.24 & $\mathbf{0 . 2 2}$ & $\mathbf{0 . 1 9}$ & 0.20 \\
\hline
\end{tabular}

Since the tie correspondences are weighted with $10 \mathrm{~cm}$ standard deviation in the adjustment and the statistics of the differences in all dimensions before and after the update are very low, area 1 shows no significant improvement (see Table 5.5).

Table 5-5 Statistics of the differences before and after the adjustment of area 1 with correspondences to the aerial oblique images [in metres].

\begin{tabular}{lccc}
\hline & $X$ & $Y$ & $Z$ \\
\hline \hline Mean & 0.003 & -0.006 & 0.001 \\
\hline Std. dev. & 0.019 & 0.018 & 0.011 \\
\hline Max & 0.038 & 0.027 & 0.054 \\
\hline Min & -0.044 & -0.056 & -0.040 \\
\hline
\end{tabular}

Area 2, however, has been updated in all three dimensions. Whereas the previous experiment using only correspondences to the aerial nadir images worsened the accuracy in every dimension, utilising aerial oblique images 
improved the accuracy in $Y$ dramatically. Overall, the combined RMSE in 3D has been improved by more than $10 \mathrm{~cm}$ (from 0.33 to 0.22 , see Table $5-8$ ).

Most correspondences to the aerial oblique data set could be identified in area 3. Although there were 95 individual triangulated tie points, all the correspondences were identified on one side of the trajectory only due to the river on the other side of the road and thus the lack of any vertical structure to derive image patches (see Figure 5.4 (blue trajectory) and Figure 5.6). This property had some impact on the statistics of the adjustment, as the trajectory has been pulled by a couple of centimetres into a positive $X$ and $Y$ direction (see Table 5.6). The accuracy, however, only improved marginally.

Table 5-6 Statistics of the differences before and after the adjustment of area 3 with correspondences to the aerial oblique images [in metres].

\begin{tabular}{lccc} 
& $\mathrm{X}$ & $\mathrm{Y}$ & $\mathrm{Z}$ \\
\hline \hline Mean & 0.017 & 0.027 & 0.013 \\
\hline Std. dev. & 0.016 & 0.017 & 0.022 \\
\hline Max & 0.042 & 0.057 & 0.041 \\
\hline Min & -0.032 & -0.007 & -0.028 \\
\hline
\end{tabular}




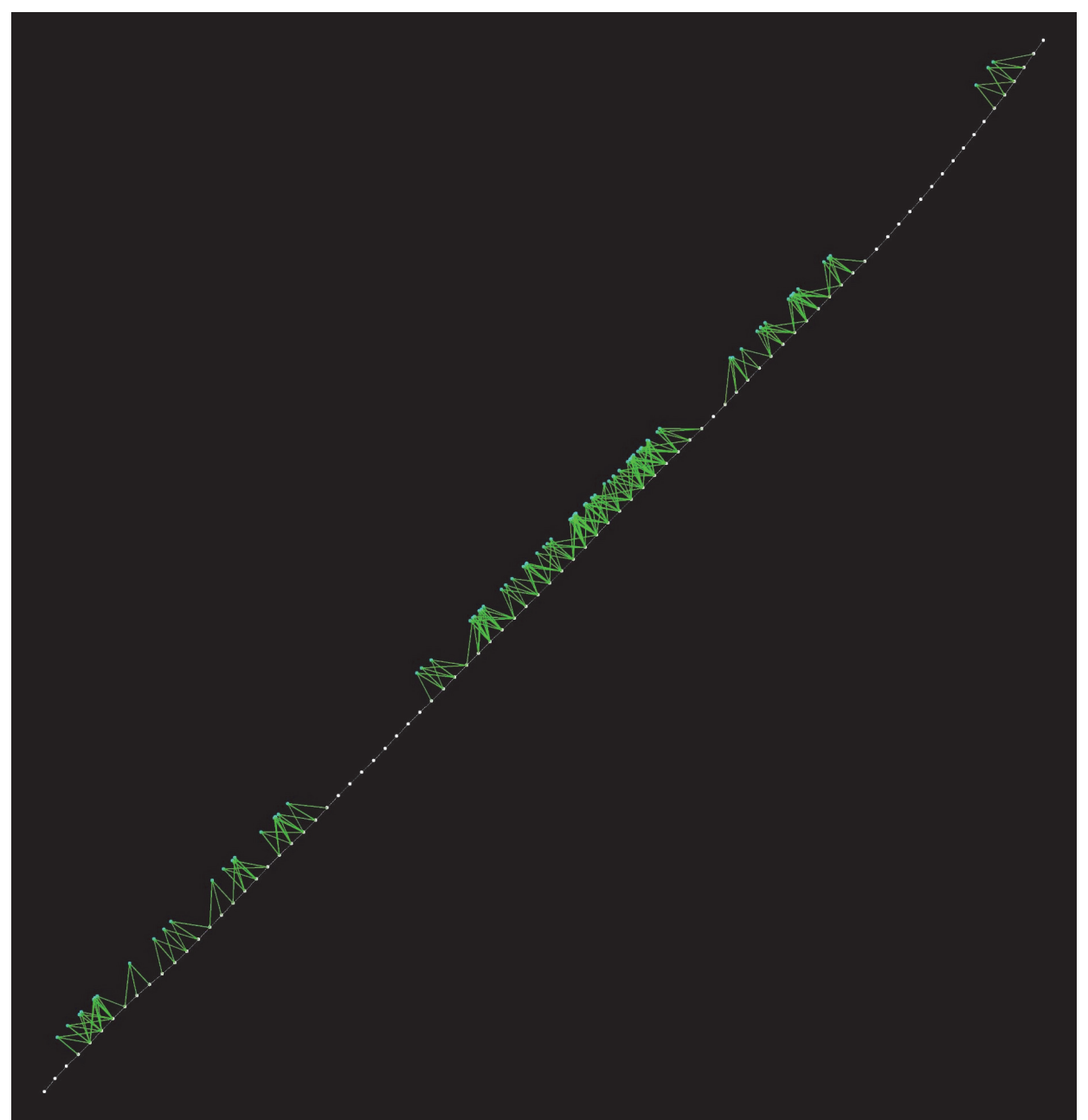

Figure 5.6 Distribution of correspondences (green) of area 3 along the trajectory (white). All the correspondences were identified on one side of the road.

\subsubsection{Adjustment results using correspondences to the aerial oblique and nadir images}

In two test areas ( 1 and 2 ), a joint adjustment was possible. To this end, the correspondences to both, the aerial nadir and oblique data set, were fed into the adjustment. This increased the number of correspondences significantly (see Table 5-1). 
Table 5-7 Adjustment result using correspondences to the aerial oblique and nadir images [in metres]. Best result in bold.

\begin{tabular}{lcccccc}
\hline Area & \multicolumn{2}{c}{ RMSE X } & \multicolumn{2}{c}{ RMSE Y } & \multicolumn{2}{c}{ RMSE Z } \\
& before & after & before & after & before & after \\
\hline \hline 1 & 0.14 & $\mathbf{0 . 1 3}$ & 0.12 & $\mathbf{0 . 0 9}$ & 0.23 & $\mathbf{0 . 2 0}$ \\
\hline 2 & $\mathbf{0 . 1 5}$ & 0.18 & 0.52 & $\mathbf{0 . 1 8}$ & $\mathbf{0 . 1 9}$ & 0.27 \\
\hline
\end{tabular}

Area 1 could be improved in every dimension, in $Y$ even to a sub-decimetre level. This is an interesting finding, as an improvement to this extent is only enabled by the combination of both nadir and oblique image correspondences.

Area 2, however, does not necessarily benefit from a joint adjustment. Although the results have improved in $X$ and $Y$ in comparison to using only correspondences to the aerial nadir images, the accuracy in $Z$ has been worsened.

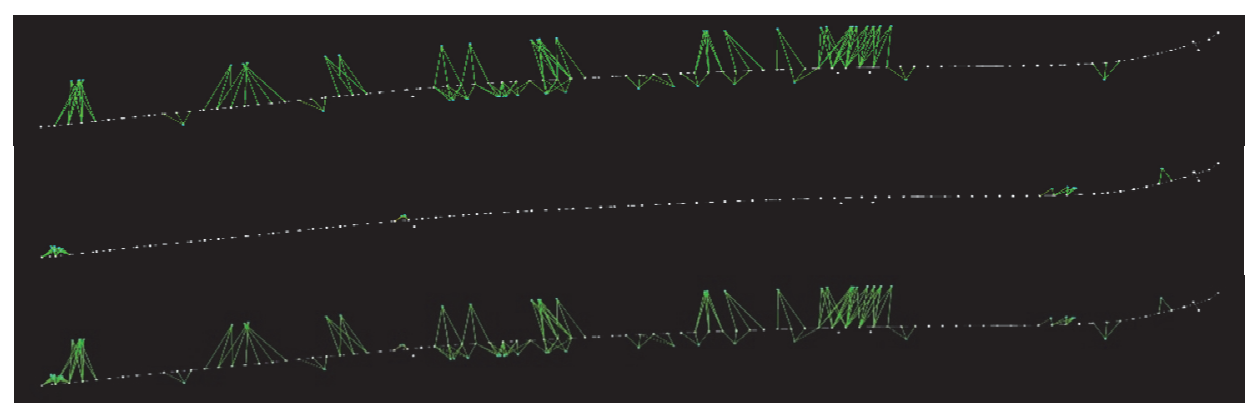

Figure 5.7 Distribution of correspondences in area 1. From top to bottom: aerial oblique correspondences, aerial nadir correspondences, and both combined.

Interestingly, the accuracy in $X$ is higher than in the single-use cases. Figure 5.7 depicts the correspondences for area 2. As mentioned in the previous section (5.5.1), the nadir correspondences in area 2 are unequally distributed and cluttered at the left hand side of the trajectory. Hence, a joint adjustment is not necessarily averaging the results obtained from a single aerial nadir or aerial oblique result. It rather depends on the number, the distribution, and the individual properties of the tie points.

\subsubsection{Summary and discussion}

The previous sections compared different adjustment scenarios utilising correspondences to aerial nadir images, aerial oblique images, or both. Table 5-8 and Table 5-9 give an overview on the overall RMSE 2D and 3D values before and after the different adjustments. 
Table 5-8 RMSE combined in $\mathrm{X}, \mathrm{Y}$ before and after respective adjustments. Best result in bold [in metres]. Borderline cases not rounded.

\begin{tabular}{lc|ccc}
\hline Area & $\begin{array}{c}\text { RMSE 2D } \\
\text { before }\end{array}$ & Nadir & Oblique & Combined \\
\hline \hline 1 & 0.13 & 0.11 & 0.13 & $\mathbf{0 . 1 1}$ \\
\hline 2 & 0.38 & 0.40 & $\mathbf{0 . 1 8}$ & $\mathbf{0 . 1 8}$ \\
\hline 3 & 0.19 & $\mathrm{n} / \mathrm{a}$ & $\mathbf{0 . 1 8}$ & $\mathrm{n} / \mathrm{a}$ \\
\hline 4 & 0.172 & $\mathbf{0 . 1 6 7}$ & $\mathrm{n} / \mathrm{a}$ & $\mathrm{n} / \mathrm{a}$ \\
\hline
\end{tabular}

Table 5-9 RMSE combined in $X, Y, Z$ before and after respective adjustments. Best result in bold [in metres]. Borderline cases not rounded.

\begin{tabular}{lc|ccc}
\hline Area & $\begin{array}{c}\text { RMSE 3D } \\
\text { before }\end{array}$ & Nadir & Oblique & Combined \\
\hline \hline 1 & 0.17 & 0.15 & 0.17 & $\mathbf{0 . 1 5}$ \\
\hline 2 & 0.33 & 0.36 & 0.22 & $\mathbf{0 . 2 1}$ \\
\hline 3 & 0.190 & n/a & $\mathbf{0 . 1 8 6}$ & $\mathrm{n} / \mathrm{a}$ \\
\hline
\end{tabular}

In general, the updates are all minor as the original recording locations already had a high accuracy. Area 2 is an exception where for instance the trajectory could be improved by almost up to $40 \mathrm{~cm}$ ( $Y$ dimension in area 2 with aerial oblique correspondences only, see Table 5-4). This ascertains the feasibility of our approach to act on deviations of the trajectory and improve or also maintain the data's accuracy up to a low decimetre level. This effect is specifically visible with respect to the horizontal accuracy (Table 5-8) which could be improved for every test area.

In particular, using correspondences to the aerial nadir images for trajectory correction is certainly a viable option as long as the height component is neglected or weighted accordingly. Interestingly, the correspondences to the oblique images could also improve or at least verify the data's accuracy, although the complexity of the aerial oblique registration pipeline is comparatively high and is more prone to outliers than the nadir registration pipeline. Future efforts are directed towards the integration of the aerial images into the bundle adjustment. Additionally, the weights ought to be set in accordance to the expected measurement accuracy while an outlier removal mechanism will avoid a worsening of the accuracy.

\subsection{Conclusion}

This section presented experimental results of an adjustment pipeline to correct mobile mapping image positions in a potentially GNSS-denied environment. Not only is the accuracy of the mobile mapping platform unknown but it may also be inaccurate. By yielding highly accurate 
correspondences between mobile mapping images and aerial nadir as well as aerial oblique images, the latter enable to act as a reference data set. To this end, image observations in the aerial data set have been triangulated and used as ground control points within a bundle adjustment. A comparison to surveyed ground control points was conducted to determine the horizontal as well as the vertical accuracy of the mobile mapping data before and after the adjustment. It could be shown that our procedure is able to verify and improve the trajectory.

\subsection{Annex - Adjustment with deteriorated data}

As the previous results could show, the original accuracy of the test trajectories is in the low decimetre range, except area 2. Area 2 depicts a narrow road overgrown by vegetation (see Figure 5.4), which potentially led to a worse positioning accuracy compared to the other areas. Interestingly, the correspondences to the aerial nadir images are not able to correct the platform's accuracy accordingly. The correspondences to the aerial oblique images, however, have a better distribution along the trajectory and the accuracy could be improved considerably after an adjustment.

In order to ascertain the potential of the procedure, an additional experiment is performed where the recording locations of the trajectory in area 2 are deteriorated by an artificial error. The error consists of an offset of $80 \mathrm{~cm}$ and a random offset of maximum 10 centimetres. Our previous findings (see 3.5.3 and 5.5) show that the vertical component of a recording location is less impacted by GNSS-induced positioning effects, and hence more stable and accurate per se and furthermore is potentially worsened by our procedure. To simulate a realistic situation, the error has been added to the horizontal dimension of a recording location only. Since the correspondences to the nadir images in area 2 are not well distributed, they do not contribute to the solution. Hence, only correspondences to the aerial oblique images are used in this case to exemplify the procedure's performance.

In contrast to previous experiments, a comparison between a horizontal and three-dimensional adjustment is conducted as well as four different methods to compute the residuals are performed. In general, mobile mapping panoramic images have a ground sampling distance, which strongly varies across the image. This may have a certain impact on the adjustment. In other words, the magnitude of a residual, which is expressed as a Euclidean distance in pixels, depends on the distance between the camera and the observed point in object space. Analogously, Xie et al. (2016) designed an asymmetric reweighting function for aerial oblique images depending on the GSD of the observation. To this end, four experiments have been devised: 
1) Residuals are not weighted

2) Residuals closer to the camera are weighted higher

$$
r=\frac{l-d(c, \hat{X})}{l} * d(x, \hat{x})
$$

3) Residuals further from the camera are weighted higher

$$
r=1-\frac{l-d(c, \hat{X})}{l} * d(x, \hat{x})
$$

4) Residuals are weighted according to the ground sampling distance of the observation

$$
r=\frac{d(x, \hat{x})}{\pi * d(c, \hat{X}) *\left(\frac{\alpha}{180}\right)}
$$

Where $d(x, \hat{x})$ denotes the computed reprojection error in the image coordinate system, $d(c, \widehat{X})$ is the distance between the camera and the observation in object space in centimetres, 1 is the maximum distance of a correspondence in object space to the camera in centimetres, $\alpha$ is the angular resolution of the panoramic camera in degrees ( 0.075 degrees in our case). The maximum distance $\mathrm{l}$ is set to 15 metres, which corresponds to a ground sampling distance of about 2 centimetres with an angular resolution of 0.075 degrees. See Table 5-10 for an example with the resulting weights.

Table 5-10 Example results for proposed residual calculation methods [in pixel].

\begin{tabular}{lccc}
\hline $\begin{array}{l}\text { Distance to } \\
\text { correspondence } \\
\text { in metres }\end{array}$ & $\begin{array}{c}\text { Residual weight } \\
\text { with method 2 }\end{array}$ & $\begin{array}{c}\text { Residual weight } \\
\text { with method 3 }\end{array}$ & $\begin{array}{c}\text { Residual weight } \\
\text { with method 4 }\end{array}$ \\
\hline 1 & 0.93 & 0.06 & 7.64 \\
\hline 3 & 0.8 & 0.2 & 2.55 \\
\hline 5 & 0.66 & 0.33 & 1.53 \\
\hline 15 & 0.33 & 0.66 & 0.76 \\
\hline
\end{tabular}

It has to be mentioned that in this particular case almost $50 \%$ of the correspondences are more than 15 metres away from the respective mobile mapping camera location. Hence, method 2 does not use these points as the weight becomes zero. Similar to the previous experiments, the correspondences are used as GCPs within the adjustment. The results for the 
horizontal adjustment are shown in Table 5.11 and for the full adjustment in Table 5-12.

Table 5-11 Results of a horizontal adjustment using different residual computation methods [in metres].

\begin{tabular}{lccccc}
\hline & Before & Method 1 & Method 2 & Method 3 & Method 4 \\
\hline RMSE X & 0.72 & $\mathbf{0 . 2 3}$ & 0.26 & 0.35 & 0.29 \\
\hline RMSE Y & 1.23 & $\mathbf{0 . 1 7}$ & 0.21 & 0.20 & 0.23 \\
\hline RMSE Z & 0.05 & $\mathbf{0 . 0 5}$ & 0.08 & 0.09 & 0.06 \\
\hline RMSE 3D & 0.82 & $\mathbf{0 . 1 7}$ & 0.20 & 0.24 & 0.22 \\
\hline
\end{tabular}

Table 5-12 Results of a full adjustment using different residual computation methods [in metres].

\begin{tabular}{lccccc}
\hline & Before & Method 1 & Method 2 & Method 3 & Method 4 \\
\hline RMSE X & 0.72 & $\mathbf{0 . 1 4}$ & 0.34 & 0.21 & 0.31 \\
\hline RMSE Y & 1.23 & 0.52 & 0.49 & $\mathbf{0 . 4 2}$ & 0.58 \\
\hline RMSE Z & 0.05 & $\mathbf{0 . 1 4}$ & 0.37 & 0.33 & 0.37 \\
\hline RMSE 3D & 0.82 & $\mathbf{0 . 3 2}$ & 0.41 & 0.33 & 0.43 \\
\hline
\end{tabular}

It is evident that all methods to compute the residuals could improve the position accuracy in area 2 . The results obtained without any specific weighting of the residuals are best and comparable to the result shown in the previous section of area 2 (see Table 5-7). The differences between the other methods are marginal, although method 2 cannot utilise about half the correspondences. Again (cf. chapter 3 ), it is noticeable that performing a full adjustment, i.e. all three dimensions, has a rather negative impact on the vertical accuracy.

It is noteworthy, however, that the absolute mean differences between the introduced correspondences of the aerial oblique data and their mobile mapping counterpart are very similar to the positioning error of the platform (see Table 5-13). This indicates that that the developed procedure returns correspondences, which are in average able to improve the positioning accuracy of the recording platform. In total, 26 correspondences, which are visible in at least 3 mobile mapping images, have been used. 
Table 5-13 Mean and standard deviation of differences between triangulated aerial oblique correspondences and mobile mapping correspondence in object space [in metres]. 26 tie points in total.

\begin{tabular}{lcccc}
\hline & $X$ & $Y$ & $Z$ & Distance in 3D \\
\hline \hline Mean difference & 0.58 & 1.26 & 0.02 & 1.42 \\
\hline SD of differences & 0.21 & 0.39 & 0.20 & 0.34
\end{tabular}


Chapter 6 - Synthesis 


\subsection{Conclusions}

The main objective of this dissertation was to investigate how aerial images can be utilised to improve or verify the accuracy of mobile mapping data in difficult positioning scenarios, such as urban areas. Terrestrial mobile mapping complements other geo-information acquisition methods by enabling a data capture perspective and accuracy unparalleled to traditional approaches. This intriguing property, however, can affect the positioning accuracy when relying on comparatively inexpensive direct sensor orientation. Compensating for this issue by using aerial images poses additional challenges. On the one hand, aerial images are a cost-efficient alternative to ground control points but on the other hand introduce major challenges with respect to their efficient utilisation as a reference. Following up on this research work's objectives, this chapter concludes the dissertation with an outlook and the impact of this contribution.

\section{Exploring different possibilities for a reliable feature extraction method}

Various obstacles had to be overcome to register mobile mapping and aerial images successfully and reliably. After investigating out-of-the-box low-level feature extraction methods, feature detection results indicated that corners have the highest repeatability between aerial nadir and mobile mapping images, in particular at road markings. Blob detection, for instance, highly relies on the surrounding of a feature point, which may appear differently in the mobile mapping and aerial nadir image. The feature description of a corner posed a challenge itself, as the illumination, the contrast, and the content is likely to vary between both image data sets. The reprojection of the mobile mapping image onto an artificial ground plane enabled the use of twodimensional image correlation methods, which are, in particular, more robust to illumination differences than feature descriptors, as our tests have shown.

In contrast, the workflow for the registration of mobile mapping with aerial oblique images was designed to rely on façade matching. Required plane estimates were derived using a sparse point cloud from mobile mapping data. The sparse point cloud was computed based on AGAST corner features. Since façades consist of many geometric elements, corner features proved to be very useful for the creation of the sparse point cloud and in subsequent steps as the object space kernel for patch creation. These object points, which mostly lie at window corners or other distinct geometric façade elements represent the centre of the computed image patches. Since these geometric elements are present in the aerial oblique images as well, a registration is more likely to succeed. 
2. Development of an accurate registration method for mobile mapping and aerial nadir imagery

A pivotal step for a successful registration between the mobile mapping and aerial nadir image was the reprojection of the mobile mapping image to an assumed ground surface. Not only did this step homogenise the perspective between the data sets, it also allowed for a resampling and a rotation of the projected mobile mapping image. This simplifies the registration substantially, as the variances between the data sets were reduced.

Another important development was the introduction of orientation parameters into the procedure. Although the error of the mobile mapping platform and hence the displacement of the projected image patch is unknown, certain strategies can be utilised to constrain the search space for correspondences. First experiments (2.2.3.2 ) used a kd-tree to identify areas where feature points in the mobile mapping image were sparse. These rather isolated points were used for an initial registration to avoid the matching of repeated patterns. In later experiments (3.4.5.2), this important step has been further improved by employing a highly constrained feature matching using KAZE. Subsequent to a Wallis filter operation, two instances of KAZE with different parameter settings for the mobile mapping and aerial nadir image are initiated. By projecting individual feature points from the mobile mapping image into the aerial nadir image, a one-to-many descriptor matching is used. This technique determines individual transformations between every mobile mapping and aerial image patch combination. Subsequently, this transformation is used to project corner points from the mobile mapping patch into the respective aerial nadir image patch for an accurate registration using phase correlation. The experiments have shown that this technique is also robust in areas where the illumination differences between the image data sets are strong.

3. Development of an accurate registration method for mobile mapping and aerial oblique imagery

In both, the aerial nadir and aerial oblique registration case, planes are hypothesised to enable a registration in the first place. As mentioned in the first objective, a sparse point cloud was computed to derive plane estimates in the scene to identify projection surfaces visible in both data sets. Additionally, an array of other techniques had to be employed to register the data sets successfully. Visibility checks needed to be undertaken to select suitable aerial oblique images as candidates for the registration. Moreover, the sampling rate of the aerial image at a certain object point needs to be computed to determine whether the resulting patch satisfies the minimum criterion for registration as well as to ascertain the spacing for the mobile mapping image patch computation. 
The actual registration is performed by mutual information, as it proved to be very suitable for our matching problems with respect to slightly varying image content and illumination differences between the patches. Besides that, it became evident that the size of the patch is an important parameter. For instance, if the patch is too large and the façade is partly occluded in the aerial oblique image, the registration may fail. If the patch is too small, the registration may converge wrongly. In any case, a hierarchical patch matching approach yielded the best results. This step has certain similarities to the initial transformation used in the aerial nadir pipeline where a first estimate supports the actual registration.

\section{Design of an adjustment technique for trajectory correction}

In collaboration with one of our partner companies, CycloMedia, one of our goals was to develop a registration pipeline, which is compatible with existing adjustment procedures. The adjustment experiments performed in the context of this thesis (Chapter $3 \&$ Chapter 5 ) relied on correspondences, which are at least visible in two aerial images. The observations in the aerial images were triangulated and used as control points within the adjustment. Theoretically, there are other possibilities as well. For instance, the integration of the aerial images into the adjustment allows for the utilisation of correspondences, which are visible in only one image. In this case, the aerial images are part of the block and their orientation elements could be fixed. The developed solution, however, ought to demonstrate primarily the potential of the developed registration procedure.

\section{Evaluation and verification of adjusted mobile mapping data}

After a successful registration and a conversion to the respective original geometries, the mobile mapping and aerial images are linked together and an adjustment can be performed. In the first experiment, the data has been artificially deteriorated whereas the data in the second experiment could be used as is. An additional experiment has been performed with an excerpt of the test data from the second experiment where the recording locations have been worsened in the horizontal dimension. The accuracy has been evaluated using surveyed ground control points as check points.

The goal to reach or maintain low decimetre accuracy has been achieved in most of the cases. In chapter 3, the focus lied on the utilisation of correspondences to aerial nadir images. The high acquisition altitude of the aerial images led to inaccuracies in the vertical dimension due to an adverse intersection geometry. The correction in the horizontal dimension, however, demonstrated that the identified and introduced correspondences could improve the positioning accuracy considerably. 
In the main part of Chapter 5, the accuracy of the mobile mapping data has not been artificially worsened. Correspondences with aerial nadir and oblique images have been used individually and in combination to ascertain the potential of both registration procedures. It became evident that both registration techniques yield accurate correspondences, as the accuracy of the mobile mapping data remained mostly unchanged. This means that the procedure is also suitable to verify the accuracy of existing mobile mapping data without the need to employ surveyed ground control points. In one particular instance of this test data set, the accuracy could be improved by the integration of aerial oblique correspondences. To determine the potential of the aerial oblique registration procedure, an additional experiment has been conducted. It could be shown that the accuracy can also be improved up to a low decimetre level.

In sum, the registration procedure is well capable to identify correspondences successfully, which are suitable for the improvement of mobile mapping imaging data, particularly in the horizontal dimension.

\subsection{Outlook}

This work discussed the development of an approach for correcting and improving mobile mapping images in difficult positioning scenarios by utilising accurate tie correspondences with aerial oblique and nadir images.

(Ultra)-wide-baseline matching is still a difficult task to automate, in particular with respect to the accuracy. What features are suitable for bridging these extremely different image geometries? In our approach, many heuristics could be used, as the task, the application, and area was clearly defined. Projecting a mobile mapping image onto a ground plane or using a sparse point cloud for finding façades in a scene are useful but are based on assumptions, which do not necessarily hold for any scenario and can be thus regarded as constraints or rather limitations of the approach. On the other hand, GNSS-induced positioning errors do mostly occur in urban areas, for which this method has been designed. Certainly, positioning issues are also present in forests or in mountainous areas but these areas were not in the scope of this project.

With the inception and maturation of machine learning methods for feature matching, one has to ask whether the presented methods are still valid in the light of future developments in this realm. This is certainly difficult to answer. If a feature suitable for tying images together can be identified under extreme geometric conditions, the aforementioned heuristics could be obviously reduced. Undoubtedly, however, the homogenisation of the data sets would also contribute to a successful registration in this case, in particular with respect to its accuracy. Or looking at it the other way round, can a procedure 
based on these heuristics be used to improve or enable machine learning methods for this task?

In any event, the issue of reliable positioning in urban areas using GNSS will persist. It is an interesting research question how emerging technologies, such as autonomous driving, autonomous drones, and other real-time services will reliably cope with this problem. A few examples of online-processing techniques have been mentioned across this thesis. A method that is able to provide high positioning accuracy reliably and consistently - comparable to our post-processing approach for mobile mapping - for these technologies is yet to be found.

After all, this work has demonstrated that the integration of image correspondences for positioning problems is a promising technique. It is possible to overcome sensor-dependent characteristics and geometric constraints; the potential of more flexible adjustment solutions, the ability to scale the problem to not only different systems but also different environments, and the utilisation of machine learning in this context would be interesting questions for future research. 


\section{Bibliography}

Alcantarilla, P.F., Bartoli, A., Davison, A.J., 2012. 'KAZE features', European Conference on Computer Vision, pp. 214-227. doi: 10.1007/978-3-64233783-3_16

Alcanterilla, P.F., Nuevo, J., Bartoli, A., 2013. 'Fast Explicit Diffusion for Accelerated Features in Nonlinear Scale Spaces', Proceedings of the British Machine Vision Conference, pp. 13.1-13.11. doi: 10.5244/C.27.13

Alsadik, B., 2016. 'A modified method for image triangulation using inclined angles', The International Archives of the Photogrammetry, Remote Sensing \& Spatial Information Sciences, 41, pp. 453-458. doi: 10.5194/isprsarchives-XLI-B3-453-2016

Angelats, E., Colomina, I., 2014. 'One step mobile mapping laser and camera data orientation and calibration', The International Archives of Photogrammetry, Remote Sensing \& Spatial Information Sciences, 40, pp. 15-20. doi: 10.5194/isprsarchives-XL-3-W1-15-2014

Azimi, S.M., Fischer, P., Körner, M., Reinartz, P., 2019. ,Aerial LaneNet: lane marking semantic segmentation in aerial imagery using wavelet-enhanced cost-sensitive symmetric fully convolutional neural networks', IEEE Transactions on Geoscience and Remote Sensing, 57, pp. 2920 - 2938. doi: 10.1109/TGRS.2018.2878510

Badino, H., Yamamoto, A., Kanade, T., 2013. 'Visual odometry by multi-frame feature integration', Proceedings of the IEEE International Conference on Computer Vision Workshops, pp. 222-229. doi: 10.1109/ICCVW.2013.37

Balazadegan Sarvrood, Y., Hosseinyalamdary, S., Gao, Y., 2016. 'Visual-LiDAR Odometry Aided by Reduced IMU', ISPRS International Journal of GeoInformation, 5.1. doi: 10.3390/ijgi5010003

Bay, H., Ess, A., Tuytelaars, T., Van Gool, L., 2006. 'Speeded-up robust features (SURF)', European conference on computer vision, pp. 404-417. doi: 10.1007/11744023_32

Beers, B., 2011. 'Collection and Application of 2D and 3D Panoramic Imagery', Proceedings of the Photogrammetric Week 2011

Berveglieri, A., Tommaselli, A.M.G., 2015. 'Locating Control Points In Aerial Images With A Multi-Scale Approach Based On Terrestrial Image Patches', The Photogrammetric Record, 30, pp. 63-81. doi: 10.1111/phor.12096

Bétaille, D., Peyret, F., Ortiz, M., Miquel, S., Godan, F., 2016. 'Improving Accuracy and Integrity with a Probabilistic Urban Trench Modeling', Navigation, 63, pp. 283-294. doi: 10.1002/navi.145

Burkhard, J., Cavegn, S., Barmettler, A., Nebiker, S., 2012. 'Stereovision mobile mapping: System design and performance evaluation', The International Archives of Photogrammetry, Remote Sensing \& Spatial Information Sciences, 39, pp. 453-458. doi: 10.5194/isprsarchives-XXXIXB5-453-2012 
Calonder, M., Lepetit, V., Strecha, C., Fua, P., 2010. 'Brief: Binary robust independent elementary features', Computer Vision-ECCV 2010, pp. 778792. doi: $10.1007 / 978-3-642-15561-1 \_56$

Carlone, L., Karaman, S., 2017. 'Attention and Anticipation in Fast VisualInertial Navigation', IEEE Transactions on Robotics, 35, pp. 1-20. doi: 10.1109/ICRA.2017.7989448

Cavegn, S., Nebiker, S., Haala, N., 2016. 'A systematic comparison of direct and image-based georeferencing in challenging urban areas', The International Archives of Photogrammetry, Remote Sensing \& Spatial Information Sciences, 41, pp. 529-536. doi: 10.5194/isprs-archives-XLIB1-529-2016

Cheng, L., Wu, Y., Tong, L., Chen, Y., Li, M., 2015. 'Hierarchical Registration Method for Airborne and Vehicle LiDAR Point Cloud', Remote Sensing, 7, pp. 13921-13944. doi: 10.3390/rs71013921

Droste, S., Jansen, T., Wegener, I., 2002. 'On the analysis of the $(1+1)$ evolutionary algorithm', Theoretical Computer Science, 276, pp. 51-81. doi: 10.1016/S0304-3975(01)00182-7

Egels, Y., Kasser, M., 2001. 'Digital Photogrammetry', Taylor \& Francis, Inc., 376 pages. doi: 10.1201/9780203305959

El-Sheimy, N., Schwarz, K., 1993. 'Kinematic positioning in three dimensions using CCD technology', Proceedings of VNIS'93-Vehicle Navigation and Information Systems Conference, pp. 472-475. doi: 10.1109/VNIS.1993.585675

Fanta-Jende, P., Nex, F., Gerke, M., Vosselman, G., 2019. 'Co-registration of panoramic mobile mapping images and oblique aerial images', The Photogrammetric Record, 34, pp. 148-173. doi: 10.1111/phor.12276

Fischer, P., Azimi, S.M., Roschlaub, R., Krauß, T., 2018. 'Towards HD Maps from Aerial Imagery: Robust Lane Marking Segmentation Using CountryScale Imagery', ISPRS International Journal of Geo-Information, 7.12. doi: 10.3390/ijgi7120458

Förstner, W., Gülch, E., 1987. 'A fast operator for detection and precise location of distinct points, corners and circular features ', Proceedings of the ISPRS Intercommission Conference on Fast Processing of Phonogrammic Data, pp. 281-305.

Geiger, A., Lenz, P., Stiller, C., Urtasun, R., 2013. 'Vision meets robotics: The KITTI dataset', The International Journal of Robotics Research, 32, pp. 12311237. doi: $10.1177 / 0278364913491297$

Godha, S., Petovello, M.G., Lachapelle, G., 2005. 'Performance Analysis of MEMS IMU/HSGPS/Magnetic Sensor Integrated System in Urban Canyons', Proceedings of the 18th International Technical Meeting of the Satellite Division of The Institute of Navigation, pp. 1977-1990.

Gontran, H., Skaloud, J., Gilliéron, P.-Y., 2007. 'A mobile mapping system for road data capture via a single camera', Advances in Mobile Mapping Technology. CRC Press, pp. 59-66. 
Groves, P.D., 2011. 'Shadow matching: A new GNSS positioning technique for urban canyons', Journal of Navigation, 64, pp. 417-430. doi: $10.1017 /$ S0373463311000087

Groves, P.D., Jiang, Z., Rudi, M., Strode, P., 2013. 'A portfolio approach to NLOS and multipath mitigation in dense urban areas', Proceedings of the 26th International Technical Meeting of The Satellite Division of the Institute of Navigation, pp. 3231-3247.

Gruen, A., Huang, X., Qin, R., Du, T., Fang, W., Boavida, J., Oliveira, A., 2013. 'Joint processing of UAV imagery and terrestrial mobile mapping system data for very high resolution city modeling', The International Archives of Photogrammetry, Remote Sensing \& Spatial Information Sciences, 40, pp. 175-182. doi: 10.5194/isprsarchives-XL-1-W2-175-2013

Gruyer, D., Belaroussi, R., Revilloud, M., 2014. 'Map-aided localization with lateral perception', Proceedings of the IEEE Intelligent Vehicles Symposium, pp. 674-680. doi: 10.1109/IVS.2014.6856528

Gu, Y.L., Hsu, L.T., Kamijo, S., 2016. 'GNSS/Onboard Inertial Sensor Integration With the Aid of 3-D Building Map for Lane-Level Vehicle SelfLocalization in Urban Canyon', IEEE Transactions on Vehicular Technology, 65, pp. 4274-4287. doi: 10.1109/TVT.2015.2497001

Gupta, A., Chang, H., Yilmaz, A., 2016. 'GPS-denied geo-localisation using visual odometry', ISPRS Ann. Photogrammetry, Remote Sensing \& Spatial Information Science, III-3, pp: 263-270. doi: 10.5194/isprs-annals-III-3263-2016

Han, J., Lo, C., 2016. 'Adaptive Time-Variant Adjustment for the Positioning Errors of a Mobile Mapping Platform in GNSS-Hostile Areas', Survey Review, 49, pp. 9-14. doi: 10.1080/00396265.2015.1104091

Harris, C., Stephens, M., 1988. 'A Combined Corner and Edge Detector', Proceedings of Fourth Alvey Vision Conference, pp. 147-151. doi: 10.1.1.231.1604

Hartley, R., Zisserman, A., 2004. 'Multiple View Geometry in Computer Vision', Cambridge University Press, 672 pages. doi: 10.1017/CBO9780511811685

Hartley, R.I., 1997. 'Lines and points in three views and the trifocal tensor', International Journal of Computer Vision, 22, pp. 125-140. doi: 10.1023/A: 1007936012022

Heinly, J., Dunn, E., Frahm, J.-M., 2012. 'Comparative evaluation of binary features', Computer Vision-ECCV 2012, pp. 759-773. doi: 10.1007/978-3642-33709-3_54

Hofmann, S., Brenner, C., 2016. 'Accuracy assessment of mobile mapping point clouds using the existing environment as terrestrial reference', The International Archives of Photogrammetry, Remote Sensing \& Spatial Information Sciences, 41, pp. 601-608. doi: 10.5194/isprsarchives-XLI-B1601-2016

Hussnain, Z., Elberink, S.O., Vosselman, G., 2016. 'Automatic feature detection, description and matching from mobile laser scanning data and 
aerial imagery', The International Archives of Photogrammetry, Remote Sensing \& Spatial Information Sciences, 41, pp. 609-616. doi: 10.5194/isprsarchives-XLI-B1-609-2016

Hussnain, Z., Oude Elberink, S., Vosselman, G., 2018. 'An automatic procedure for mobile laser scanning platform 6dof trajectory adjustment', The International Archives of Photogrammetry, Remote Sensing \& Spatial Information Sciences, 42, pp. 203-209. doi: 10.5194/isprs-archives-XLII-1203-2018

Irish, A., Iland, D., Madhow, U., 2015. 'Urban Localization and 3D Mapping Using GNSS Shadows', https://insidegnss.com/urban-localization-and-3dmapping-using-gnss-shadows/ (accessed on August $5^{\text {th }}, 2019$ )

Jaud, M., Rouveure, R., Faure, P., Monod, M.-O., 2013. 'Methods for FMCW radar map georeferencing', ISPRS Journal of Photogrammetry and Remote Sensing, 84, pp: 33-42. doi: 10.1016/j.isprsjprs.2013.07.002

Javanmardi, M., Javanmardi, E., Gu, Y., Kamijo, S., 2017. 'Towards HighDefinition 3D Urban Mapping: Road Feature-Based Registration of Mobile Mapping Systems and Aerial Imagery', Remote Sensing, 9. doi: $10.3390 /$ rs9100975

Jende, P., Hussnain, Z., Peter, M., Oude Elberink, S., Gerke, M., Vosselman, G., 2016a. 'Low-Level Tie Feature Extraction of Mobile Mapping Data (MLS/Images) and Aerial Imagery', The International Archives of Photogrammetry, Remote Sensing \& Spatial Information Sciences, 40, pp. 19-26. doi: 10.5194/isprs-archives-XL-3-W4-19-2016

Jende, P., Nex, F., Gerke, M., Vosselman, G., 2017. 'Fully automatic featurebased registration of mobile mapping and aerial nadir images for enabling the adjustment of mobile platform locations in gnss-denied urban environments', The International Archives of Photogrammetry, Remote Sensing \& Spatial Information Sciences, 42, pp. 317-323. doi: 10.5194/isprsarchives-XLII-1-W1-317-2017

Jende, P., Nex, F., Gerke, M., Vosselman, G., 2018a. 'A fully automatic approach to register mobile mapping and airborne imagery to support the correction of platform trajectories in GNSS-denied urban areas', ISPRS Journal of Photogrammetry and Remote Sensing, 141, pp. 86-99. doi: 10.1016/j.isprsjprs.2018.04.017

Jende, P., Nex, F., Gerke, M., Vosselman, G., 2018b. 'A guided registration strategy employing virtual planes to overcome non-standard geometries using the example of mobile mapping and aerial oblique imagery', The International Archives of Photogrammetry, Remote Sensing \& Spatial Information Sciences, 42, pp. 471-477. doi: 10.5194/isprs-archives-XLII-2471-2018

Jende, P., Peter, M., Gerke, M., Vosselman, G., 2016b. 'Advanced tie feature matching for the registration of mobile mapping imaging data and aerial imagery', The International Archives of Photogrammetry, Remote Sensing \& 
Spatial Information Sciences, 41, pp. 617-623. doi: 10.5194/isprs-archivesXLI-B1-617-2016

Ji, S., Shi, Y., Shan, J., Shao, X., Shi, Z., Yuan, X., Yang, P., Wu, W., Tang, H., Shibasaki, R., 2015. 'Particle filtering methods for georeferencing panoramic image sequence in complex urban scenes', ISPRS Journal of Photogrammetry and Remote Sensing, 105, pp. 1-12. doi: 10.1016/j.isprsjprs.2015.03.005

Jia, Y., Li, M., An, L., Zhang, X., 2003. 'Autonomous navigation of a miniature mobile robot using real-time trinocular stereo machine', IEEE International Conference on Robotics, Intelligent Systems and Signal Processing, pp. 417421. doi: 10.1109/RISSP.2003.1285610

Kersting, A., Habib, A., Rau, J., 2012. 'New method for the calibration of multicamera mobile mapping systems', The International Archives of Photogrammetry, Remote Sensing \& Spatial Information Sciences 39, pp. 121-126. doi: 10.5194/isprsarchives-XXXIX-B1-121-2012

Kos, T., Markezic, I., Pokrajcic, J., 2010. 'Effects of multipath reception on GPS positioning performance', Proceedings ELMAR-2010, pp. 399-402.

Köthe, U., 2003a. 'Edge and junction detection with an improved structure tensor', Pattern Recognition. DAGM 2003, pp. 25-32. doi: 10.1007/978-3540-45243-0_4

Köthe, U., 2003b. 'Gradient-Based Segmentation Requires Doubling of the Sampling Rate', Tech. Rep. FBI-HH-M326/03, University of Hamburg, Informatics Dept.

Kümmerle, R., Steder, B., Dornhege, C., Kleiner, A., Grisetti, G., Burgard, W., 2011. 'Large scale graph-based SLAM using aerial images as prior information', Autonomous Robots, 30, pp: 25-39. doi: 10.1007/s10514-0109204-1

Leung, K.Y.K., Clark, C.M., Huissoon, J.P., 2008. 'Localization in urban environments by matching ground level video images with an aerial image', Proceedings of IEEE International Conference on Robotics and Automation, pp. 551-556. doi: 10.1109/ROBOT.2008.4543264

Levi, G., Hassner, T., 2015. 'LATCH: Learned Arrangments of Three Patch Codes', Proceedings of IEEE Winter Conference on Applications of Computer Vision, pp. 1-9. doi: 10.1109/WACV.2016.7477723

Levinson, J., Thrun, S., 2007. 'Map-Based Precision Vehicle Localization in Urban Environments', Robotics: Science and Systems, 4, p. 1. doi: 10.15607/RSS.2007.III.016

Lin, T.-Y., Cui, Y., Belongie, S., Hays, J., 2015. 'Learning deep representations for ground-to-aerial geolocalization', Proceedings of the IEEE Conference on Computer Vision and Pattern Recognition (CVPR), pp. 5007-5015. doi: 10.1109/CVPR.2015.7299135

Lowe, D.G., 1985. 'Perceptual Organization and Visual Recognition', Kluwer Academic Publishers, 153 pages. doi: 10.1145/24667.1058032 
Lowe, D.G., 2004. 'Distinctive image features from scale-invariant keypoints', International Journal of Computer Vision, 60, pp. 91-110. doi: 10.1023/B:VISI.0000029664.99615.94

Mair, E., Hager, G.D., Burschka, D., Suppa, M., Hirzinger, G., 2010. 'Adaptive and generic corner detection based on the accelerated segment test', Computer Vision-ECCV 2010, pp. 183-196. doi: https://doi.org/10.1007/978-3-642-15552-9_14

Mattes, D., Haynor, D.R., Vesselle, H., Lewellyn, T.K., Eubank, W., 2001. 'Nonrigid multimodality image registration', Proceedings of Medical Imaging. doi: https://doi.org/10.1117/12.431046

Melekhov, I., Ylioinas, J., Kannala, J., Rahtu, E., 2017. 'Relative camera pose estimation using convolutional neural networks', Proceedings of the International Conference on Advanced Concepts for Intelligent Vision Systems, pp. 675-687. doi: 10.1007/978-3-319-70353-4_57

Migliore, D., Rigamonti, R., Marzorati, D., Matteucci, M., Sorrenti, D.G., 2009. 'Use a single camera for simultaneous localization and mapping with mobile object tracking in dynamic environments', ICRA Workshop on Safe navigation in open and dynamic environments: Application to autonomous vehicles, pp. 12-17.

Miksik, O., Mikolajczyk, K., 2012. 'Evaluation of Local Detectors and Descriptors for Fast Feature Matching', Proceedings of the International Conference on Pattern Recognition, pp. 2681-2684.

Molina, P., Blázquez, M., Cucci, D., Colomina, I., 2017. 'First Results of a Tandem Terrestrial-Unmanned Aerial mapKITE System with Kinematic Ground Control Points for Corridor Mapping', Remote Sensing, 9. doi: $10.3390 /$ rs9010060

Morel, J.-M., Yu, G., 2009. 'ASIFT: A New Framework for Fully Affine Invariant Image Comparison', SIAM Journal on Imaging Sciences, 2, pp. 438-469. doi: https://doi.org/10.1137/080732730

Muad, A.M., Hussain, A., Samad, S.A., Mustaffa, M.M., Majlis, B.Y., 2004. 'Implementation of inverse perspective mapping algorithm for the development of an automatic lane tracking system', Proceedings of the IEEE Region 10 Conference, TENCON 2004, pp. 207-210. doi: 10.1109/TENCON.2004.1414393

Novatel, 2014. 'IMU Errors and Their Effects', Technical Report, 6 pages,https://www.novatel.com/assets/Documents/Bulletins/APN064.pdf (accessed on August $5^{\text {th }}, 2019$ )

Payá, L., Gil, A., Reinoso, O., 2017. 'A state-of-the-art review on mapping and localization of mobile robots using omnidirectional vision sensors', Journal of Sensors, 2017, 20 pages. doi: 10.1155/2017/3497650

Pink, O., Stiller, C., 2010. 'Automated map generation from aerial images for precise vehicle localization', Proceedings of the Intelligent Transportation Systems (ITSC) Conference, pp. 1517-1522. doi: 10.1109/ITSC.2010.5625276 
Pomerleau, F., Colas, F., Siegwart, R., Magnenat, S., 2013. 'Comparing ICP variants on real-world data sets', Autonomous Robots, 34, pp. 133-148. doi: 10.1007/s10514-013-9327-2

Puente, I., González-Jorge, H., Martínez-Sánchez, J., Arias, P., 2013. 'Review of mobile mapping and surveying technologies', Measurement, 46, pp. 21272145. doi: 10.1016/j.measurement.2013.03.006

Ramakrishnan, S., Waters, D.W., Balakrishnan, J., 2013. 'Robust Wi-Fi Assisted GNSS Positioning in Urban Canyons', Proceedings of the 26th International Technical Meeting of The Satellite Division of the Institute of Navigation, pp. 2058-2065.

Reddy, B.S., Chatterji, B.N., 1996. 'An FFT-based technique for translation, rotation, and scale-invariant image registration', IEEE transactions on image processing, 5, pp. 1266-1271. doi: 10.1109/83.506761

Roh, H., Jeong, J., Cho, Y., Kim, A., 2016. 'Accurate Mobile Urban Mapping via Digital Map-Based SLAM', Sensors, 16. doi: 10.3390/s16081315

Rosten, E., Drummond, T., 2006. 'Machine learning for high-speed corner detection', Computer Vision-ECCV 2006, pp. 430-443. doi: 10.1007/11744023_34

Roth, L., Kuhn, A., Mayer, H., 2017. 'Wide-Baseline Image Matching with Projective View Synthesis and Calibrated Geometric Verification', PFG Journal of Photogrammetry, Remote Sensing and Geoinformation Science, 85, pp. 85-95. doi: 10.1007/s41064-017-0012-5

Rublee, E., Rabaud, V., Konolige, K., Bradski, G., 2011. 'ORB: an efficient alternative to SIFT or SURF', Computer Vision-ICCV 2011, pp. 2564-2571. doi: 10.1109/ICCV.2011.6126544

Scaramuzza, D., 2014. 'Omnidirectional camera', Computer Vision: A Reference Guide, pp. 552-560. doi: 10.5167/uzh-106115

Schindler, A., 2013. 'Vehicle self-localization with high-precision digital maps', Proceedings of the IEEE Intelligent Vehicles Symposium, pp. 141-146. doi: 10.1109/IVS.2013.6629461

Shan, Q., Wu, C., Curless, B., Furukawa, Y., Hernandez, C., Seitzy, S.M., 2014. 'Accurate Geo-registration by Ground-to-Aerial Image Matching', Proceedings of the 2014 2nd International Conference on 3D Vision, 1, pp. 525-532. doi: 10.1109/3DV.2014.69

Snyder, J.P., 1993. 'Flattening the earth: two thousand years of map projections', University of Chicago Press, 384 pages. doi: 10.14714/CP31.651

Strode, P.R., Groves, P.D., 2016. 'GNSS multipath detection using threefrequency signal-to-noise measurements', GPS solutions, 20, 3, pp. 399-412. doi: $10.1007 /$ s10291-015-0449-1

Tao, C.V., 2000. 'Mobile mapping technology for road network data acquisition', Journal of Geospatial Engineering, 2, 2, pp. 1-13.

Tao, C.V., Li, J., 2007. 'Advances in mobile mapping technology', CRC Press, 176 pages, ISBN 9780415427234 
Tola, E., Lepetit, V., Fua, P., 2010. 'Daisy: An efficient dense descriptor applied to wide-baseline stereo', IEEE Transactions on Pattern Analysis and Machine Intelligence, 32, 5, pp. 815-830. doi: 10.1109/TPAMI.2009.77

Torr, P.H., Zisserman, A., 2000. 'MLESAC: A new robust estimator with application to estimating image geometry', Computer vision and image understanding, 78, 1, pp. 138-156. doi: 10.1006/cviu.1999.0832

Tournaire, O., Soheilian, B., Paparoditis, N., 2006. 'Towards a Sub-Decimetric Georeferencing of Ground-Based Mobile Mapping Systems in Urban Areas: Matching Ground-Based and Aerial-based Imagery Using Roadmarks', The International Archives of Photogrammetry, Remote Sensing \& Spatial Information Sciences, ISPRS Commission I Symposium "From Sensors to Imagery".

van den Heuvel, F.A., Verwaal, R., Beers, B., 2006. 'Calibration of fisheye camera systems and the reduction of chromatic aberration'. The International Archives of Photogrammetry, Remote Sensing \& Spatial Information Sciences, 36, 5, pp. 1-6.

Wallis, R.H., 1976. 'An Approach to the Space Variant Restoration and Enhancement of Images', Proceedings of the Symposium on Current Mathematical Problems in Image Science.

Wan, X., Liu, J.G., Yan, H., 2015. 'The Illumination Robustness of Phase Correlation for Image Alignment', IEEE Transactions on Geoscience and Remote Sensing, 53, 10, pp. 5746-5759. doi: 10.1109/TGRS.2015.2429740

Wilson, J.L., 2016. 'Automotive WiFi Availability in Dynamic Urban Canyon Environments', Navigation, 63, 2, pp. 161-172. doi: 10.1002/navi.137

Wu, B., Xie, L., Hu, H., Zhu, Q., Yau, E., 2018. 'Integration of aerial oblique imagery and terrestrial imagery for optimized 3D modeling in urban areas', ISPRS Journal of Photogrammetry and Remote Sensing, 139, pp. 119-132. doi: 10.1016/j.isprsjprs.2018.03.004

Xie, L., Hu, H., Wang, J., Zhu, Q., Chen, M., 2016. 'An asymmetric re-weighting method for the precision combined bundle adjustment of aerial oblique images', ISPRS Journal of Photogrammetry and Remote Sensing, 117, pp. 92-107. doi: 10.1016/j.isprsjprs.2016.03.017

Yang, X., Cheng, K.-T., 2012. 'LDB: An ultra-fast feature for scalable augmented reality on mobile devices', Proceedings of the IEEE International Symposium on Mixed and Augmented Reality, pp. 49-57. doi: 10.1109/ISMAR.2012.6402537

Yicong Tian, C.C., Mubarak Shah, 2017. 'Cross-View Image Matching for Geolocalization in Urban Environments', Proceedings of the IEEE Conference on Computer Vision and Pattern Recognition, pp. 3608-3616. doi: 10.1109/CVPR.2017.216

Yixiang Tian, M.G., George Vosselman, Qing Zhu, 2014. 'Automatic Edge Matching Across an Image Sequence Based on Reliable Points', The International Archives of Photogrammetry, Remote Sensing \& Spatial Information Sciences, 37, pp. 657-662. 
Zagoruyko, S., Komodakis, N., 2015. 'Learning to compare image patches via convolutional neural networks', Proceedings of the IEEE Conference on Computer Vision and Pattern Recognition, pp. 4353-4361. doi: 10.1109/CVPR.2015.7299064

Zhang, J., Singh, S., 2015. 'Visual-lidar odometry and mapping: low-drift, robust, and fast', Proceedings of the IEEE International Conference on Robotics and Automation, pp. 2174-2181. doi: 10.1109/ICRA.2015.7139486 


\section{Summary}

Mobile mapping has become an important extension to traditional geoinformation acquisition techniques. Its unique ability to record street-level data using laser scanners or cameras on a large scale complements and augments the photogrammetric portfolio. Particularly in urban areas, MM plays a significant role, as it enables complementary data representations of the scene in conjunction with other data-capture solutions.

In general, urban areas pose a challenge with respect to satellite-based positioning solutions due to high-rise buildings and other tall structures in builtup areas which may obstruct the direct-line-of-sight to navigation satellites. As a consequence, multipath and non-line-of-sight effects may occur and impede the position estimation of the receiver.

Although MM platforms usually utilise inertial navigation to bridge potential signal outages (i.e. non-line-of-sight), a complete mitigation of these effects remains an unsolved problem, as the discrimination between a valid and an invalid signal (i.e. multipath) from navigation satellites is per se not possible. Hence, the accuracy of the MM platform's position and thus its acquired data is unknown and likely impaired in such scenarios. Although the traditional approach to introduce ground truth into the correction procedure by using ground control points has been proven reliable and exact, the acquisition of such information is costly and labour-intensive. In the light of a growing market and a diversification of geo-data acquisition platforms, automated, costefficient, and scalable solutions are greatly demanded.

To this end, the present research investigates the possibility to integrate external ground truth derived from aerial images into the position estimation of the MM platform in order (1) to verify and (2) to improve its accuracy if possible.

Aerial images are a standard product in many countries and are acquired at regular intervals at a nationwide scale. The effects which hinder a reliable position estimation of MM platforms are not applicable to aircrafts, as the direct line-of-sight to navigation satellites is usually not obstructed. Calibrated cameras, highly accurate inertial sensors, and positioning equipment enable precise sensor orientation and thus the recording of high-resolution nadir as well as oblique imagery with great accuracy.

In order to utilise aerial images for the verification and improvement of MM data, both data sets need to be registered first. The MM data used for this research project are panoramic images, which are recorded in a discrete manner along the platform's trajectory. Since the aerial and the MM image 
data set do not share the same perspective on the scene, many characteristics between the images are different and have a significant influence on the registration quality. This poses the main challenge of the present work.

The first chapter outlines the background, the research problem and objectives, the second chapter of the thesis focuses on exploring different techniques for a co-registration of aerial nadir and MM images. The first part of chapter two investigates the possibility to use low-level tie features for the registration task between aerial nadir and MM panoramic images. In order to simplify the correspondence problem, MM images are reprojected on an artificial ground surface, i.e. to simulate a top-down view similar to the aerial nadir image counterpart. The actual registration is based on low-level feature matching, which can be coarsely separated in a feature detection and description phase. To this end, different feature matching methods are compared with each other based on their performance to identify and register image features that are visible in both - the MM and the aerial nadir - data sets. Conducted experiments indicate that correspondences can be mostly identified at road markings. As road markings may be repetitive (e.g. zebra crossings), their feature descriptions can be ambiguous, which consequently leads to wrong correspondences.

These findings lead to a number of considerations, which are discussed in the second part of chapter two. Although MM images have an unknown accuracy, a certain margin of error can be assumed. To this end, the orientation parameters of the MM images are used to constrain the search space for correspondences. In order to exploit this property, orientation parameters are employed in conjunction with template matching. A kd-tree ${ }^{21}$ is used to identify isolated points in order to avoid problems with repeated patterns in the MM image. These points are then projected into the aerial image using the MM orientation parameters. The window size of the template matching accounts for possible localisation errors of the platform while constraining the search for correspondences. Subsequently, these initial correspondences are used to compute a transformation between the image pair to reliably map the remaining (and possibly cluttered) points from one image into the other to enable an accurate registration. The experiments ascertain great improvements of the inlier rate compared to the previous approach. In certain scenarios, however, repeated patterns still cause ambiguities. Moreover, other differences between the images, e.g. illumination, contrast, and content may influence the performance of the registration procedure. 
The third chapter outlines the development of an automatic and reliable coregistration procedure between aerial nadir and MM images for the adjustment of MM data. Based on previous insights, the procedure is further developed with respect to automated processing and to account for the overall image differences between aerial and MM images. This is achieved by utilising MM orientation parameters for the computation of an initial transformation which is verified for plausibility. Subsequently, this transformation is used to project salient corner points from the MM image into the aerial image. The exact correspondence is identified by using the phase information of image templates. With an inlier rate of about $98 \%$, the co-registration technique proves to be successful. The image observations of the correspondences in the aerial nadir images are triangulated and used for the adjustment of the MM data. This approach is able to verify as well as improve the horizontal error of the MM data up to a decimetre. The vertical dimension, however, is prone to be worsened by this approach, as the aerial images have been acquired at a high altitude that potentially leads to an unfavourable intersection geometry.

Although aerial nadir and MM images have a different perspective on the scene, overlapping areas can be found mostly on the ground where road markings and other ground-based features can be identified as valid correspondences. Since not all roads have road markings or other distinct features on the ground which can be easily registered, the fourth chapter focusses on exploring the possibility to register MM images with aerial oblique images. Similar to the previous registration case, aerial oblique images do not share the same perspective on the scene with MM images. Mutual entities between both data sets are, however, façades and other vertical surfaces along the road. In order to utilise these areas for registration, though, another reprojection of the MM images is required. Unlike the ground, façade planes cannot be easily assumed. Hence, a sparse point cloud from MM images is generated to fit planes into the scene in order to create façade hypotheses. These surfaces can then be discretised in object space to generate image patches of both, the aerial oblique and the MM data. This step overcomes the large perspective differences between the two data sets dramatically and simplifies the registration problem. A thorough experimental section using difficult registration scenarios ascertains an inlier rate of about $80 \%$.

Aerial oblique images bear a great potential for the adjustment task at hand. Not only can the number of correspondences between the aerial and MM data set be increased but it also allows for correspondences at different height levels which can potentially stabilise the geometry. Chapter five presents adjustment results for four different areas in Rotterdam showcasing the performance of the aerial nadir as well as the aerial oblique registration approach in various scenarios. The verification of the results 
using surveyed GCPs demonstrates that depending on the area and the setting low decimetre accuracy is well achievable.

In general, this research investigates the use of aerial images for the correction of MM imaging data. The development of novel techniques to deal with this non-standard registration problem is the focus of this research effort. The combination of image reprojection mechanics, guided matching strategies, and illumination-invariant similarity measures enables the identification of highly accurate correspondences between the aerial and the terrestrial data sets at hand. Since aerial images are widely available, frequently updated, and sensor systems are becoming more powerful, presented techniques demonstrate the feasibility to overcome geometric differences efficiently. Solving positioning issues in urban areas is not solely a research problem for terrestrial mapping but also for closely related fields and technologies, such as robotics, UAV photogrammetry, or autonomous driving. The utilisation of visual cues for the correction of platform trajectories is not only a viable but also a cost-efficient and accurate method, which may well experience a more widespread use in the future. 


\section{Samenvatting}

Mobiele kartering is een belangrijke uitbreiding geworden van de traditionele geo-informatieverwervingstechnieken. De unieke mogelijkheid om gegevens op straatniveau op te nemen met behulp van laserscanners of camera's op grote schaal breidt het fotogrammetrisch portfolio uit. Met name in stedelijke gebieden speelt mobiele kartering (MK) een belangrijke rol, omdat het complementaire representaties van een scène mogelijk maakt in combinatie met andere oplossingen voor gegevensinwinning.

In het algemeen vormen stedelijke gebieden een uitdaging met betrekking tot plaatsbepalingsoplossingen op basis van satellieten als gevolg van hoogbouw en andere hoge structuren in bebouwde gebieden, die de directe zichtlijn naar navigatiesatellieten kunnen belemmeren. Als gevolg hiervan kunnen multipath-effecten en occlusie optreden, die de positiebepaling van de ontvanger kunnen bemoeilijken.

Hoewel MK-platforms gewoonlijk gebruik maken van traagheidsnavigatie om potentiële signaaluitval te overbruggen, blifft een volledige beperking van de effecten een onopgelost probleem, aangezien het onderscheid tussen een geldig en een ongeldig signaal (d.w.z. multi-path) van navigatiesatellieten op zich niet mogelijk is. Daarom is de nauwkeurigheid van de positie van het MKplatform en dus ook van de verkregen gegevens onbekend en in dergelijke scenario's waarschijnlijk beperkt. Hoewel de traditionele aanpak om paspunten in de correctieprocedure te gebruiken als betrouwbaar en nauwkeurig bekend is, is het verkrijgen van dergelijke informatie kostbaar en arbeidsintensief. In het licht van een groeiende markt en een diversificatie van platforms voor gegevensinwinning is er veel vraag naar geautomatiseerde, kostenefficiënte en schaalbare oplossingen.

Daartoe onderzoekt dit werk de mogelijkheid om externe referentiegegevens afgeleid uit luchtfoto's te integreren in de positiebepaling van het MK-platform om (1) de nauwkeurigheid ervan te verifiëren en (2) indien mogelijk te verbeteren.

Luchtfoto's zijn in veel landen een standaardproduct en worden met regelmatige tussenpozen op landelijke schaal verkregen. De problemen, die een betrouwbare positiebepaling van MK-platforms belemmeren, zijn niet van toepassing op vliegtuigen, aangezien de directe zichtlijn naar navigatiesatellieten doorgaans niet wordt belemmerd. Gekalibreerde camera's, zeer nauwkeurige traagheidsnavigatiesensoren en positioneringsapparatuur maken een nauwkeurige sensororiëntatie en daarmee de opname van hogeresolutie nadir- en oblieke beelden met grote nauwkeurigheid mogelijk. 
Om luchtfoto's te kunnen gebruiken voor de verificatie en verbetering van MKgegevens, moeten beide datasets eerst worden geregistreerd. De MKgegevens die voor dit onderzoeksproject worden gebruikt zijn panoramische beelden, die op vele locaties langs het traject van het platform worden opgenomen. Aangezien de luchtfoto's en de MK-foto's niet hetzelfde perspectief hebben op de scène, zijn veel kenmerken tussen de beelden verschillend en hebben ze een aanzienlijke invloed op de registratiekwaliteit. Dit vormt de belangrijkste uitdaging van het huidige werk.

Het eerste hoofdstuk schetst de achtergrond, het onderzoeksprobleem en de doelstellingen. Het tweede hoofdstuk van het proefschrift richt zich op het verkennen van verschillende technieken voor een co-registratie van luchtfoto's en MK-beelden. Het eerste deel van hoofdstuk twee onderzoekt de mogelijkheid om low-level kenmerken te gebruiken voor de registratietaak tussen luchtfoto's en MK-panoramabeelden. Om het correspondentieprobleem te vereenvoudigen, worden MK-beelden geprojecteerd op een kunstmatig grondoppervlak, d.w.z. om een top-downweergave te simuleren die vergelijkbaar is met die van een luchtfoto. De eigenlijke registratie is gebaseerd op een matching van de kenmerken, die grof kan worden gescheiden in een fase van kenmerkdetectie en -beschrijving. Daartoe worden verschillende methoden voor het matchen van functies met elkaar vergeleken op basis van hun prestaties om beeldkenmerken te identificeren en te registreren die zichtbaar zijn in zowel de MK- als het luchtfoto-datasets. Uit uitgevoerde experimenten blijkt dat overeenkomsten meestal bij wegmarkeringen kunnen worden geïdentificeerd. Omdat wegmarkeringen zich kunnen herhalen (bv. zebrapaden), kunnen de beschrijvingen van de kenmerken ervan dubbelzinnig zijn, wat tot verkeerde correspondentie kan leiden.

Deze bevindingen leiden tot een aantal overwegingen, die in het tweede deel van hoofdstuk twee worden besproken. Hoewel MK-beelden een onbekende nauwkeurigheid hebben, kan een bepaalde foutmarge worden aangenomen. Hiertoe worden de oriënteringsparameters van de MK-afbeeldingen gebruikt om de zoekruimte voor correspondentie te beperken. Om deze eigenschap te exploiteren, worden oriënteringsparameters gebruikt in combinatie met het matchen van patronen. Een kd-tree ${ }^{22}$ wordt gebruikt om geïsoleerde punten te identificeren om problemen met herhaalde patronen in het MK-beeld te voorkomen. Deze punten worden vervolgens geprojecteerd in de luchtfoto met behulp van de MK-oriëntatieparameters. De zoekruimte voor corresponderende punten is bij de matching is afgestemd op de grootte van mogelijke lokalisatiefouten van het platform. Vervolgens worden deze initiële correspondenties gebruikt om een transformatie tussen het beeldpaar te 
berekenen om de resterende (en mogelijk geclusterde) punten betrouwbaar van het ene beeld naar het andere over te dragen om een nauwkeurige registratie mogelijk te maken. Uit de experimenten blijkt dat het slaagpercentage van de matching ten opzichte van de vorige aanpak aanzienlijk is verbeterd. In bepaalde scenario's veroorzaken herhaalde patronen echter nog steeds meerduidigheden. Bovendien kunnen andere verschillen tussen de beelden, zoals die in verlichting, contrast en inhoud, van invloed zijn op de werking van de registratieprocedure.

Het derde hoofdstuk beschrijft de ontwikkeling van een automatische en betrouwbare co-registratieprocedure tussen luchtfoto's en MKbeelden voor de aanpassing van MK-gegevens. Op basis van eerdere inzichten wordt de procedure verder ontwikkeld met betrekking tot de geautomatiseerde verwerking en om rekening te houden met de totale beeldverschillen tussen lucht- en MK-beelden. Dit wordt bereikt door gebruik te maken van MK-oriëntatieparameters voor de berekening van een initiële transformatie die op plausibiliteit wordt geverifieerd. Vervolgens wordt deze transformatie gebruikt om opvallende hoekpunten in het MK-beeld te projecteren naar het luchtfoto. De exacte correspondentie wordt geïdentificeerd door gebruik te maken van de fase-informatie van beeldkenmerken. Met een slaagpercentage van ongeveer $98 \%$ blijkt de coregistratietechniek succesvol te zijn. De beeldpunten van de correspondenties in de nadirluchtfoto's worden getrianguleerd en gebruikt voor de aanpassing van de MK-gegevens. Deze aanpak is in staat om de horizontale fout van de MK-gegevens tot op een decimeter nauwkeurig te controleren en te verbeteren. De verticale dimensie wordt door deze benadering echter vaak verslechterd, omdat de luchtfoto's op grote hoogte zijn verkregen, wat mogelijk tot ongunstige snijdingshoeken leidt.

Hoewel luchtfoto's en MK-beelden een ander perspectief op de scène hebben, kunnen overlappende gebieden meestal op de grond worden gevonden waar wegmarkeringen en andere kenmerken op de grond als geldige overeenkomsten kunnen worden geïdentificeerd. Aangezien niet alle wegen zijn voorzien van wegmarkeringen of andere duidelijke kenmerken op de grond die gemakkelijk kunnen worden geregistreerd, wordt in het vierde hoofdstuk ingegaan op de mogelijkheid om de MK-beelden met oblieke luchtfoto's te registreren. Net als bij de vorige registratietaak, delen oblieke luchtfoto's niet hetzelfde perspectief op de scène met MK-beelden. Gevels en andere verticale vlakken langs de weg zijn echter in beide soorten van beelden zichtbaar. Om deze gebieden te gebruiken voor registratie is echter een andere projectie van de MK-beelden vereist. In tegenstelling tot een grondvlak zijn gevelvlakken niet eenvoudig aan te nemen. Daarom wordt een ijle puntenwolk van MK-beelden gegenereerd om vlakken in de scène te passen en zo gevelhypothesen te creëren. Deze oppervlakken kunnen vervolgens worden 
gediscretiseerd in de objectruimte om beeldpatches te genereren van zowel de oblieke luchtfoto's als de MK-gegevens. Deze stap reduceert de grote perspectiefverschillen tussen de twee datasets drastisch en vereenvoudigt het registratieprobleem. Een grondig experimenteel gedeelte waarin gebruik wordt gemaakt van moeilijke registratiescenario's stelt een lager percentage van ongeveer $80 \%$ vast.

De oblieke luchtfoto's hebben een groot potentieel voor de onderhavige aanpassingstaak. Niet alleen kan het aantal correspondenties tussen de luchtfoto's en de MK-dataset worden verhoogd, maar het maakt ook correspondenties op verschillende hoogteniveaus mogelijk, die de geometrie kunnen stabiliseren. In hoofdstuk vijf worden de resultaten van de aanpassingen voor vier verschillende gebieden in Rotterdam gepresenteerd, waarbij de prestaties met de nadir luchtfoto's en de oblieke luchtfoto's in verschillende scenario's worden getoond. De verificatie van de resultaten met behulp van de onderzochte checkpunten toont aan dat, afhankelijk van het gebied en de instelling, een lage decimeternauwkeurigheid goed haalbaar is.

In het algemeen onderzoekt dit werk het gebruik van luchtfoto's voor de correctie van MK-beeldgegevens. De ontwikkeling van nieuwe technieken om dit niet-standaard registratieprobleem aan te pakken staat centraal in dit onderzoek. De combinatie van beeldprojecties, matchingstrategieën en belichtingsinvariante criteria voor de beoordeling van matchingresultaten maakt het mogelijk om zeer nauwkeurige overeenkomsten te identificeren tussen de luchtfoto's en terrestrische datasets. Aangezien luchtfoto's op grote schaal beschikbaar zijn, vaak worden bijgewerkt en sensorsystemen steeds krachtiger worden, tonen de gepresenteerde technieken de haalbaarheid aan om efficiënt met de geometrische verschillen om te gaan. Het oplossen van plaatsbepalingsproblemen in stedelijke gebieden is niet alleen een onderzoeksprobleem voor het in kaart brengen van het land, maar ook voor nauwverwante gebieden en technologieën, zoals robotica, UAVfotogrammetrie of autonoom rijden. Het gebruik van visuele signalen voor de correctie van platformtrajecten is niet alleen een haalbare maar ook een kostenefficiënte en nauwkeurige methode, die in de toekomst wellicht op grotere schaal zal worden toegepast. 